\title{
A Single Vesicle Assay to Study the Electrochemical Gradient Regulation in Glutamatergic and GABAergic Synaptic Vesicles
}

PhD Thesis

\author{
in partial fulfilment of the requirements \\ for the degree "Doctor of Philosophy (PhD)" \\ in the Neuroscience Program \\ at the Georg August University Göttingen \\ Faculty of Biology
}

submitted by

Zohreh Farsi

born in

Varamin, Iran

Göttingen, 2015 
Thesis committee members:

Supervisor, reviewer:

Prof. Dr. Reinhard Jahn

Department of Neurobiology

Max-Planck Institute for Biophysical Chemistry Göttingen, Germany

PhD committee member, reviewer:

\section{Prof. Dr. Silvio O. Rizzoli}

Department of Neuro- and Sensory Physiology University of Medical Center Göttingen Göttingen, Germany

PhD committee member:

Prof. Dr. Tobias Moser

Institute for Auditory Neuroscience and Inner Ear lab University of Medical Center Göttingen

Göttingen, Germany

$\mathrm{PhD}$ committee member:

\section{Dr. Geert van den Bogaart}

Department of Tumor Immunology Radboud University Medical Center Nijmegen, The Netherlands

Date of the oral examination: November 26, 2015 
I hereby declare that I prepared this PhD thesis, entitled "A Single Vesicle Assay to Study the Electrochemical Gradient Regulation in Glutamatergic and GABAergic Synaptic Vesicles", on my own and with no other sources and aids than those cited.

Zohreh Farsi

September 2015, Göttingen 
تقديم به يدر و مادرم

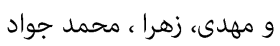




\section{Table of Contents}

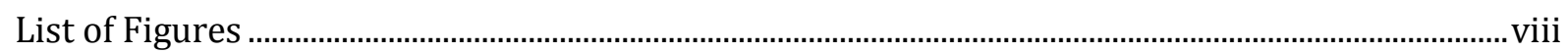

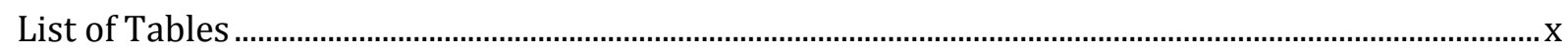

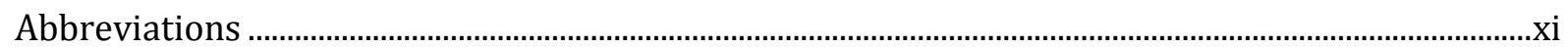

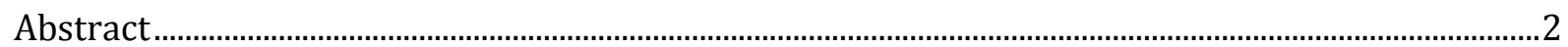

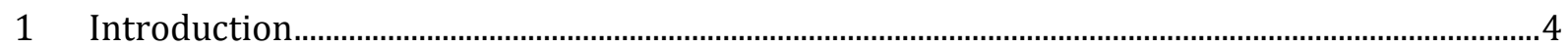

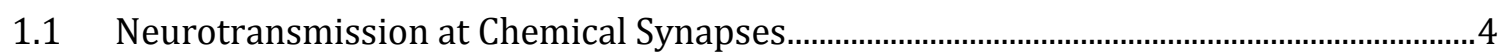

1.1.1 Synaptic Vesicle Recycling.......................................................................................

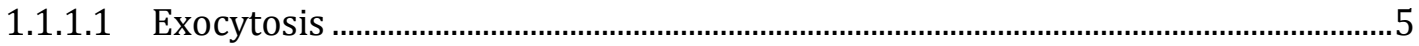

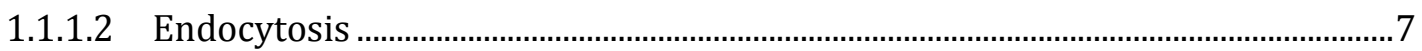

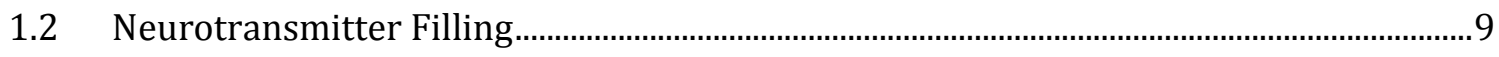

1.2.1 Electrochemical Gradient Formation and Regulation.................................................10

1.2.2 Neurotransmitter Uptake ............................................................................................... 14

1.2.2.1 Regulation of the Cytoplasmic Concentrations of Neurotransmitters............... 14

1.2.2.2 Vesicular Neurotransmitter Transporters .............................................................. 17

1.2.2.3 Transport Mechanism and Regulation of VGLUT and VGAT ................................19

1.3 Physiological Importance of Neurotransmitter Filling ................................................2 22

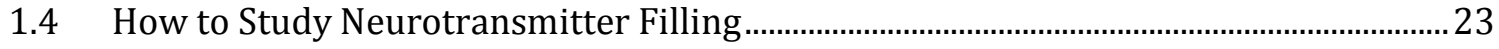

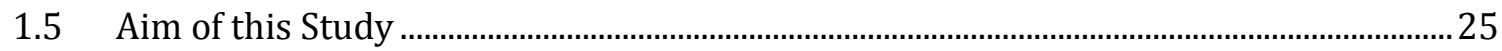

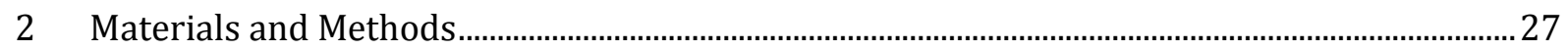

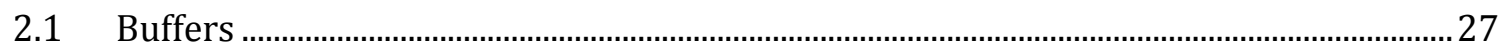

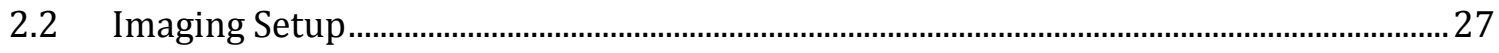

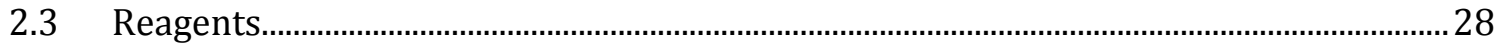

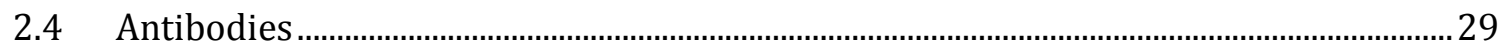

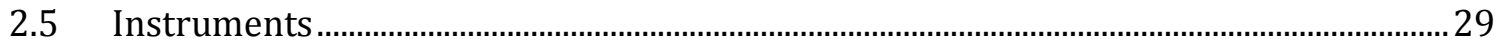

2.6 Developing a Single Vesicle Assay..................................................................................... 31

2.6.1 Isolation of Synaptic Vesicles ..................................................................................... 31

2.6.2 Immobilization of Synaptic Vesicles.............................................................................. 33

2.6.3 Imaging of Synaptic Vesicles............................................................................................ 34

2.6.3.1 Optical Alignment in TIRF Setup .......................................................................... 34

2.6.3.2 Coupling a UV Flash Lamp to TIRF Setup..................................................................... 36

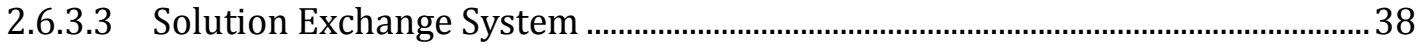


2.6.4 Probe Optimization ................................................................................................. 38

2.6.4.1 pH Sensitive Probes ....................................................................................................... 41

2.6.4.2 Potentiometric Probes............................................................................................. 52

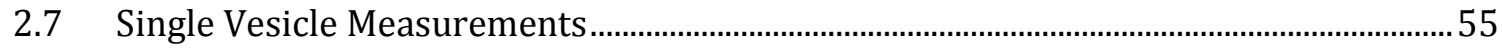

2.7.1 Single Vesicle Acidification Assay................................................................................... 55

2.7.2 Single Vesicle Potentiometric Assay...…………………………………………….... 56

2.7.3 Antibody Labeling of Synaptic Vesicles...................................................................... 57

2.7.4 Buffering Capacity Measurements .................................................................................57

2.7.5 Proton Permeability Measurements ............................................................................ 58

2.7.6 Data Analysis.............................................................................................................. 59

2.7.6.1 Image Analysis of Single SVs ………….................................................................. 59

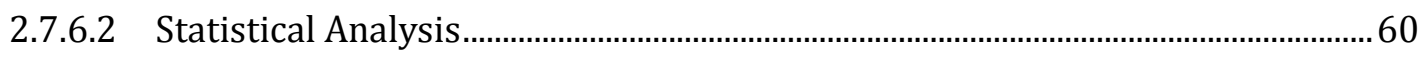

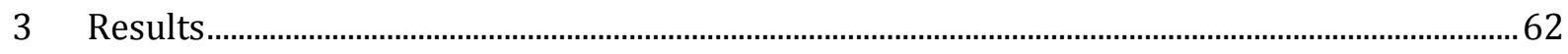

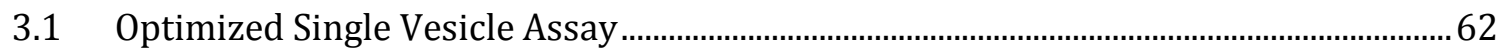

3.2 Electrochemical Gradient at different ATP Concentrations ................................................. 65

3.2.1 $\Delta \mathrm{pH}$ and $\Delta \psi$ in Glutamatergic and GABAergic SVs ...................................................68

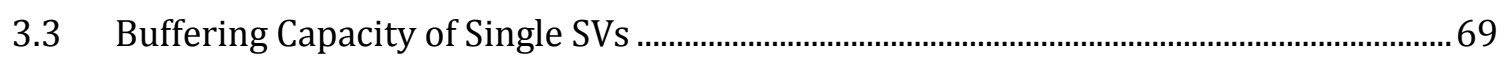

3.4 Proton Permeability of Single SVs...................................................................................... 71

3.5 Effect of Regulatory Ions on the Electrochemical Gradient.................................................. 76

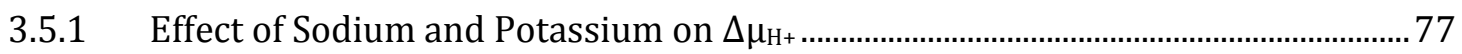

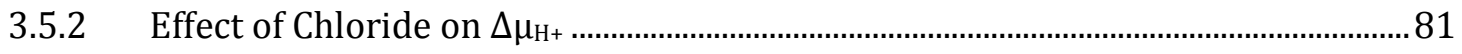

3.6 Effect of Neurotransmitters on Electrochemical Gradient .................................................. 84

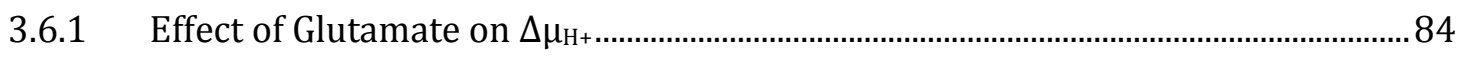

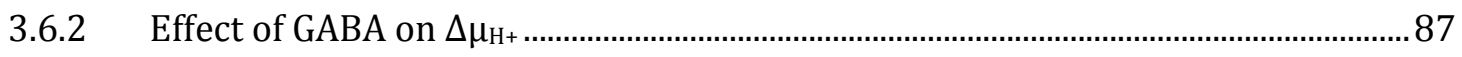

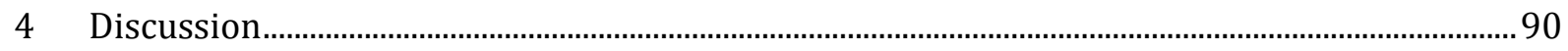

$4.1 \quad$ The Relationship between $\Delta \mathrm{pH}$ and $\Delta \psi$ Is not Linear ..................................................... 90

4.2 Synaptic Vesicles Acidify in less than One Second ................................................................91

4.3 Glutamatergic SVs Generate Larger Electrochemical Gradient Compared to

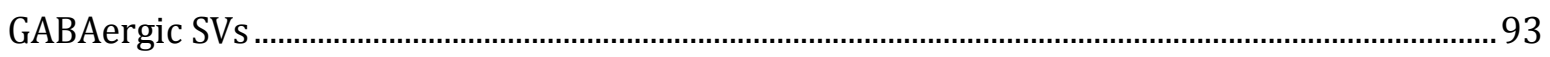

4.4 VGAT Functions as a GABA/H+ Antiporter ...................................................................... 97

4.4.1 Regulation of Luminal Cl- in GABAergic SVs after Endocytosis................................ 99

4.4.2 Transport Mechanism of VGAT ……………….............................................................. 100

4.5 VGLUT Transports Chloride and Potassium ................................................................... 101 
4.6 $\mathrm{Na}^{+} / \mathrm{H}^{+}$Exchange Stimulates Vesicle Loading in both Glutamatergic and GABAergic

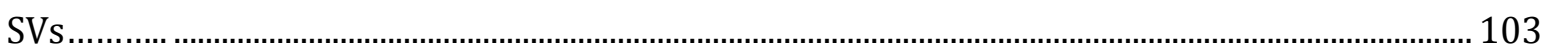

$4.7 \quad$ Stoichiometry of $\mathrm{K}^{+} / \mathrm{H}^{+}$and $\mathrm{Na}^{+} / \mathrm{H}^{+}$Exchange............................................................. 104

4.8 Neurotransmitters in the Lumen Stabilize $\Delta \mu_{\mathrm{H}+}$ across the Membrane of SVs............ 105

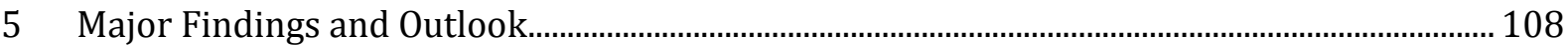

5.1 Vesicular Transporters Significantly Contribute to Regulation of their Driving

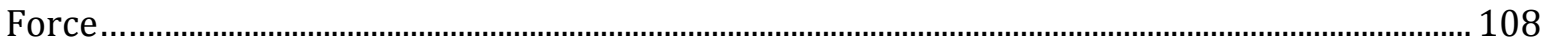

5.2 Single Vesicle Assay Reports Subtle Differences with high Precision............................. 109

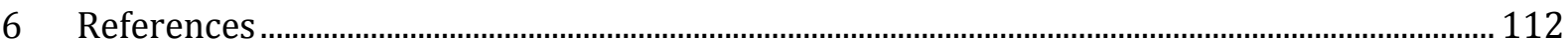

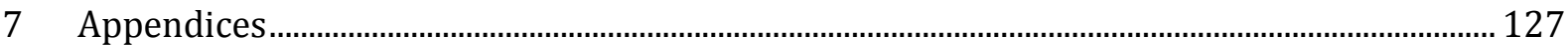

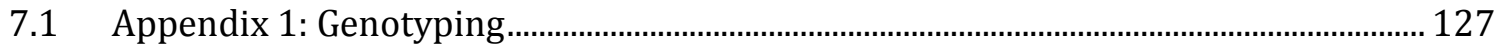

7.2 Appendix 2: Western Blotting and Dot Blotting.................................................................. 128

7.3 Appendix 3: MATLAB Code for Spot Detection ................................................................. 129

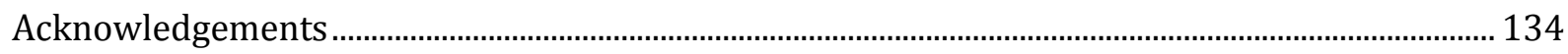

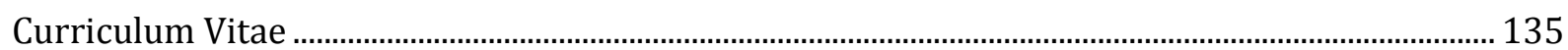




\section{List of Figures}

Figure 1-1 Sequence of events at the presynaptic terminal of a chemical synapse........................

Figure 1-2 Vesicular transporters...................................................................................................19

Figure 2-1 Purification of SVs from rat brains............................................................................ 31

Figure 2-2 Optimization of the purification protocol for purifying SVs from mouse brain....... 33

Figure 2-3 Laser alignment in TIRF setup...................................................................................... 35

Figure 2-4 Widefield versus TIRF illumination............................................................................. 36

Figure 2-5 Coupling UV light to the setup through a side opening of the microscope.................. 37

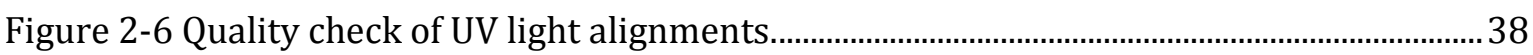

Figure 2-7 Effect of $\mathrm{p} K_{\mathrm{a}}$ of a $\mathrm{pH}$-sensitive probe on its fluorescence response to $\mathrm{pH}$ change from 7.4 to 5.6 .

Figure 2-8 Response of LysoTracker Red DND-99 and LysoSensor Green DND-189 to SV

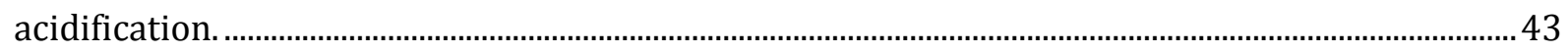

Figure 2-9 Acridine Orange mechanism and its response to acidification. .................................... 45

Figure 2-10 Loading of Pyranine into SVs via fusion with liposomes containing dye. ................ 46

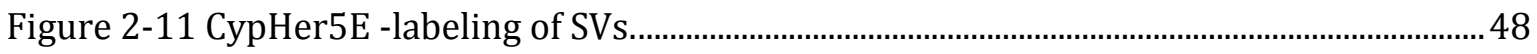

Figure 2-12 Spectral characteristics of $\mathrm{spH}$............................................................................... 50

Figure 2-13 Quantification of the number of fluorophores in single SVs....................................... 51

Figure 2-14 spH as a pH probe for single vesicle acidification assay............................................. 52

Figure 2-15 VF2.1.Cl as the appropriate potentiometric probe for single vesicle assay............54

Figure 2-16 Measuring the initial pH of lumen of spH-SVs............................................................. 56

Figure 3-1 Imaging setup for single vesicle imaging....................................................................... 62

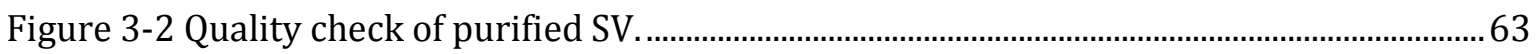

Figure 3-3 Distinguishing glutamatergic and GABAergic SVs by antibody staining.................... 64

Figure 3-4 Luminal $\mathrm{pH}$ of single spH-SVs after acidification at different ATP concentrations. 66

Figure 3-5 Changes in membrane potential across the membrane upon acidification at different ATP.

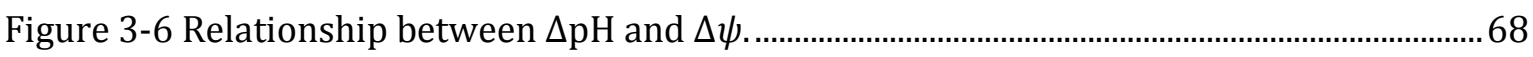

Figure 3-7 Comparison between glutamatergic and GABAergic vesicles in their proton electrochemical gradient. ................................................................................................................. 69

Figure 3-8 Buffering capacity measurement................................................................................ 70

Figure 3-9 Relationship between buffering capacity and luminal pH. ............................................ 71

Figure 3-10 Proton efflux measurements...................................................................................... 72

Figure 3-11 Comparison between glutamatergic and GABAergic SVs in proton permeability.

Figure 3-12 Size distribution of glutamatergic and GABAergic SVs.............................................. 74

Figure 3-13 Correlation between antibody-labeling intensity and luminal $\mathrm{pH}$ in GABAergic SVs.

Figure 3-14 Effect of GABA on proton efflux from GABAergic SVs. ................................................. 76

Figure 3-15 Effect of $\mathrm{K}^{+}$and $\mathrm{Na}^{+}$on the $\mathrm{pH}$ gradient across the membrane of SVs.......................77

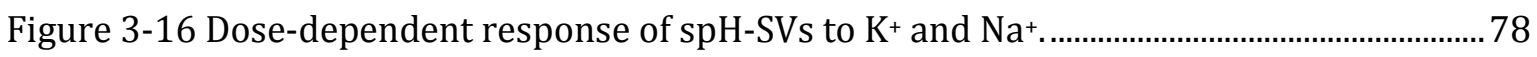


Figure 3-17 Inhibition of $\mathrm{Na}^{+} / \mathrm{K}^{+}$alkalinization by EIPA

Figure 3-18 $\mathrm{Na}^{+} / \mathrm{K}^{+-}$-induced alkalinization in glutamatergic and GABAergic SVs.

Figure 3-19 Effect of $\mathrm{Na}^{+}$and $\mathrm{K}^{+}$transport on the membrane potential across the membrane of SVs.

Figure 3-20 Effect of chloride on the $\mathrm{pH}$ gradient across the membrane. . .82

Figure 3-21 Chloride-induced acidification in glutamatergic and GABAergic SVs........................ 83

Figure 3-22 Effect of chloride on the membrane potential of SVs................................................... 84

Figure 3-23 Effect of glutamate on the pH gradient of SVs. ............................................................. 85

Figure 3-24 Effect of glutamate on membrane potential across the membrane.............................86

Figure 3-25 Buffering effect of glutamate..................................................................................... 87

Figure 3-26 Effect of GABA on the pH gradient of SVs.................................................................. 88

Figure 3-27 Effect of GABA on the membrane potential across the membrane of SVs..............89

Figure 4-1 Two versions of alternating access for membrane transporters. ................................. 95

Figure 4-2 Model of GABA/H $\mathrm{H}^{+}$antiport by VGAT.......................................................................... 101

Figure 5-1 Regulation of the balance between the two components of $\Delta \mu \mathrm{H}^{+}$in glutamatergic

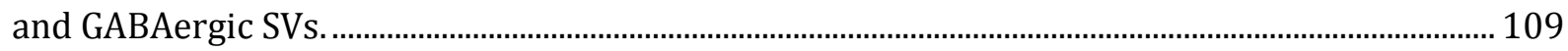

Figure 7-1 Genotyping of spH-21 transgenic mice. .................................................................... 128 


\section{List of Tables}

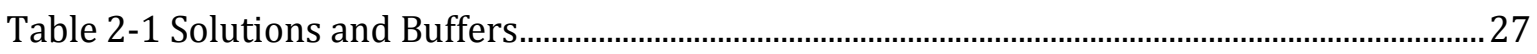

Table 2-2 Microscope and Other Equipment of Imaging Setup..................................................... 27

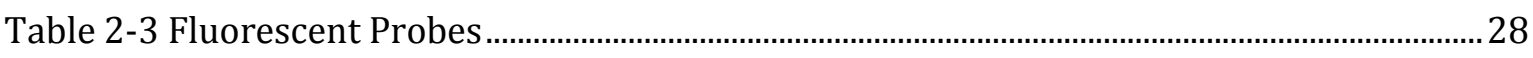

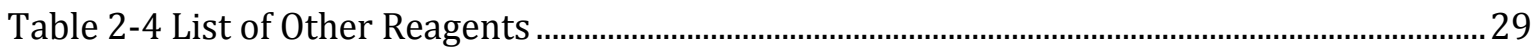

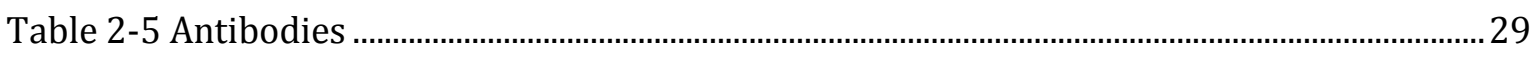

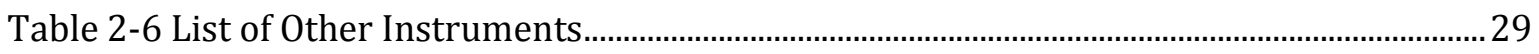

Table 2-7 List of $\mathrm{pH} /$ potentiometric probes tested in this study …………………...................... 40

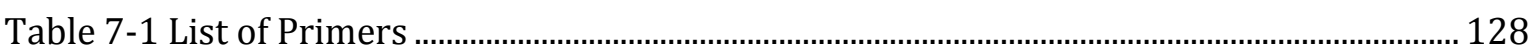




\section{Abbreviations}

AO

CCD

$\mathrm{ClC}$

CME

CNS

CPG

EAAT

EIPA

ER

FCCP

GABA

GFP

NHE

NPE

NPT

PBS

PLL

pmf

RIM

SD

SEM

SLC

SNARE

SNR

spH

SV

TCA cycle

TEA

TIRF

VAchT

V-ATPase

VAMP

VEAT

VGAT

VGlut

VMAT

VNUT
Acridine orange

Charge-coupled device

$\mathrm{Cl}^{-}$channel/transporter

Clathrin-mediated endocytosis

Central nervous system

Controlled-pore glass

Excitatory amino acid transporter

5-(N-Ethyl-N-isopropyl)amiloride

Endoplasmic reticulum

carbonyl cyanide-p-trifluoromethoxyphenylhydrazone

$\Upsilon$-Amino butyric Acid

Green fluorescent protein

$\mathrm{Na}^{+} / \mathrm{H}^{+}$Exchanger

5-(N-Ethyl-N-isopropyl)amiloride

$\mathrm{Na}^{+} /$Pi Transporter

Phosphate buffer saline

Poly-L-Lysine

Proton motive force

Rab3-interacting molecule

Standard deviation

Standard error of mean

Solute carrier

Soluble $N$-ethylmaleimide-sensitive factor attachment protein receptors

Signal-to-Noise Ratio

Super-ecliptic pHluorin

Synaptic vesicle

Tricaboxylic acid cycle

Tetraethylammonium

Total internal reflection fluorescence

Vesicular acetylcholine Transporter

Vacuolar adenosine 5'-triphosphatase

Vesicular-associated membrane protein

Vesicular excitatory amino acid transporter

Vesicular GABA transporter

Vesicular glutamate transporter

Vesicular monoamine transporter

Vesicular nucleotide transporter 


\section{Abstract}

During neuronal activity, synapses sustain neurotransmission by a high fidelity multistep process called synaptic vesicle (SV) recycling. This process involves endocytosis, neurotransmitter loading and exocytosis of SVs within a timeframe lasting from a few seconds to tens of seconds at different synapses. Despite extensive studies on endo/exocytosis, there is a scarcity of details regarding vesicle loading and its regulation.

Vesicle filling requires two distinct but dependent processes. First, the vacuolar $\mathrm{H}^{+}-$ ATPase (V-ATPase) builds a concentration gradient $(\Delta \mathrm{pH})$ as well as an electrical potential $(\Delta \psi)$ across the membrane of SVs by pumping of protons into the lumen of the vesicle at the expense of ATP. Vesicular transporters then use this combined electrochemical gradient $\left(\Delta \mu_{H^{+}}\right)$to drive the loading of transmitters into the SVs.

Neurotransmitter molecules are differently charged at neutral $\mathrm{pH}$, and although $\Delta \mu_{\mathrm{H}+}$ is required for their transport, the contribution of $\Delta \mathrm{pH}$ and $\Delta \psi$ to their transport is different and depends on their charge. For positively charged monoamines and acetylcholine, $\Delta \mathrm{pH}$ provides the main driving force. In case of negatively charged glutamate, $\Delta \psi$ is more important and for neutral GABA, both components of $\Delta \mu_{\mathrm{H}+}$ play equal roles. Therefore, accumulation of massive amounts of either of these transmitters within the short lifetime of a recycling SV would demand additional compensating mechanisms to maintain the right balance between $\Delta \mathrm{pH}$ and $\Delta \psi$ during each cycle of neurotransmitter uptake.

Existing models so far have proposed that uptake of these transmitters are probably associated with the compensating ion fluxes which are either mediated by the transporter itself or provided by other ion exchangers present on SVs. However, there are still major disagreements. Moreover, whether these compensating mechanisms are different in distinct vesicles, and if this is the case, the molecular mechanisms underlying these differences are still enigmatic, particularly when considering that SVs share the majority of their molecular composition.

In the current study, a novel single vesicle assay was developed to first explore the basic questions about the extent and kinetics of the two components of $\Delta \mu_{\mathrm{H}+}$ at the single vesicle level, and second, to unravel how the balance between $\Delta \mathrm{pH}$ and $\Delta \psi$ is regulated in glutamatergic and GABAergic SVs, which have different bioenergetics requirements. 
In this assay, SVs purified from transgenic mice expressing super-ecliptic pHluorin in the vesicular lumen (spH-SVs) were imaged using TIRF (total-internal reflection fluorescence) microscopy to accurately measure luminal $\mathrm{pH}$ changes above $\mathrm{pH}$ 6. In addition, SVs were labeled with voltage sensitive dye VF2.1.Cl to quantitatively measure changes in membrane potential across the lipid bilayer of single SVs for the first time. After measuring $\Delta \mathrm{pH}$ or $\Delta \psi$, antibody labeling against VGAT or VGLUT1 allowed for unequivocally distinguishing GABAergic from glutamatergic SVs.

This study provides evidence that SVs can acidify with sub-second kinetics and their biophysical characteristics such as buffering capacity and proton permeability fall within the range of reported values for other trafficking organelles. Moreover, a detailed comparison of $\Delta \mathrm{pH}$ and $\Delta \psi$ in glutamatergic and GABAergic SVs at the single vesicle level revealed that regulatory mechanisms do exist to optimize the balance of the electrochemical gradient, and that the vesicular transporter itself bears responsibility. It was demonstrated that VGAT transports GABA with a GABA/ $\mathrm{H}^{+}$anitport mechanism. This transport mechanism enables VGAT to keep the balance between the two components of $\Delta \mu_{\mathrm{H}_{+}}$during neurotransmitter loading. In addition, it was shown that VGLUT can transport $\mathrm{Cl}^{-}$and also functions as a $\mathrm{K}^{+} / \mathrm{H}^{+}$exchanger, both of which assist the transporter to tailor the balance to greater $\Delta \psi$ which is the main driving force for glutamate uptake. Together, these findings introduce vesicular transporters as novel regulators of the electrochemical gradient, which would grant additional significance to their role in synaptic transmission regulation. 


\section{Introduction}

\subsection{Neurotransmission at Chemical Synapses}

A single neuron is composed of three major compartments: the cell body, also called the perikaryon or soma, where the nucleus, protein synthesis machinery and several other organelles are located, dendrites, which provide a large surface to receive synaptic inputs from other nerve cells by forming extensive branches called 'dendritic trees', and axons, which are long processes built to conduct nerve impulses from the cell body to the other nerve cells. Axonal branches possess morphologically distinct regions called boutons, which are club-shaped enlargements very close to the cell body or the dendrites of other neurons (Brodal, 1992). The place of close contact between two neurons where the neuronal signals are transmitted is called a synapse, and the narrow space between the two neurons is called the synaptic cleft. Two types of synapses can be distinguished based on their mechanism of transmission: electrical synapses and chemical synapses. At the electrical synapses, the two communicating neurons are linked together by gap junctions, which consist of precisely aligned channels that span the synaptic cleft (Pereda, 2014). On the other hand, at chemical synapses, the synaptic cleft is substantially wider and the information is transmitted via release of chemical signals called neurotransmitters from the presynaptic neuron. Prior to release, the neurotransmitter molecules are stored in spherical, membrane-bounded organelles called synaptic vesicles. The main feature of chemical synapses, which are the most abundant type of synapse in the nervous system, is the accumulation of synaptic vesicles (SVs) near the presynaptic membrane of boutons (Purves, 2012).

The sequence of events involved in transmission at a chemical synapse is as follows (Südhof, 2004): First, a nerve impulse in the form of a brief electrical discharge, an action potential, invades the presynaptic bouton, and depolarizes the presynaptic membrane by perturbing the distribution of positively and negatively charged particles across the membrane. The membrane depolarization leads to opening of voltagesensitive $\mathrm{Ca}^{2+}$ channels, and this in turn elevates the presynaptic $\mathrm{Ca}^{2+}$ concentration by influx of extracellular $\mathrm{Ca}^{2+}$. This triggers the SVs to fuse with the presynaptic membrane, a process called exocytosis, which results in release of their neurotransmitter contents into the synaptic cleft. The neurotransmitter molecules then diffuse across the synaptic 
cleft and bind to specific receptors at the postsynaptic membrane. There are two types of postsynaptic receptors, which differ in their structure and mechanism of transducing neurotransmitter binding into a postsynaptic response. One type is the ionotropic receptors, which contain a membrane-spanning domain that forms a ion channel. The second type is the metabotropic receptors, which do not have ion channels as part of their structure. Neurotransmitter binding to these receptors activates intermediate molecules called G-proteins, which interact either directly with ion channels or with other effector proteins that regulate ion channels (Purves, 2012). Activation of either of these receptors leads directly (in case of ionotropic receptors) or indirectly (in case of metabotropic receptors) to a transient alteration in the postsynaptic membrane permeability to certain ions, which in turn triggers a subsequent sequence of events at the postsynaptic neuron. On the other hand, at presynaptic boutons a process called synaptic vesicle recycling occurs following SV exocytosis (Südhof, 2004).

\subsubsection{Synaptic Vesicle Recycling}

At the presynaptic terminals, $\mathrm{Ca}^{2+}$-triggered SV exocytosis is followed by retrieval of SV components from the presynaptic membrane via a process called endocytosis. This results in the formation of new SVs which can sequester neurotransmitter molecules and undergo a new round of release upon arrival of subsequent impulses. This trafficking cycle, called synaptic vesicle recycling, enables the synapse to sustain neurotransmission particularly in response to high-intensity synaptic inputs (Neher, 2010). One complete round of SV recycling can be elaborated in three sequential main steps: endocytosis, neurotransmitter filling, and exocytosis (Figure 1-1). In this section, a brief overview of endo/exocytosis will be presented and the process of neurotransmitter filling, which is the main focus of this thesis, will be discussed in more detail in the next section.

\subsubsection{Exocytosis}

Fusion of SVs with the presynaptic membrane occurs preferentially at specialized regions of the presynaptic membrane called active zones, which are juxtaposed to a region of postsynaptic plasma membrane where neurotransmitter receptors are aggregated (Dittman and Ryan, 2009). 
As a general scheme of SV exocytosis, it is assumed that vesicles follow a series of sequential steps (Figure 1-1): they first translocate to the active zone, which can be accelerated by scaffolding proteins like bassoon (Hallermann et al., 2010), and dock to the free release sites, where Rab3 on the SVs interacts with RIMs (Rab-3 interacting molecules) (Deng et al., 2011). Docking is then followed by a priming step in which SVs undergo some preparatory reactions which make them fusion-competent. At the molecular level, a network of interactions among many proteins mediates docking and priming and it is rather difficult to assign a particular protein exclusively to one of these two steps. Among these proteins, SNARE proteins (soluble $N$-ethylmaleimide-sensitive factor (NSF) attachment protein receptors) are the crucial players (Südhof and Rizo, 2011). Synaptobrevin (also called vesicle associated membrane protein or VAMP), which is located on SVs, together with syntaxin-1 and SNAP-25 on the presynaptic plasma membrane, form an $\alpha$-helical trans-SNARE complex (SNAREs reside on two opposing membranes) to exert the force required for pulling the SV and presynaptic membranes tightly together (Jahn and Fasshauer, 2012).

Beside SNAREs, Munc13 and Munc18-1, whose absence result in complete loss of neurotransmitter release are other key components of the fusion machinery (Varoqueaux et al., 2002; Verhage et al., 2000). These proteins are proposed to initiate trans-SNARE complex assembly by binding to syntaxin-1 and providing a template to bring the three SNAREs together (Ma et al., 2013). Finally, primed SVs are fused with the plasma membrane either by a trigger (calcium) or spontaneously (Verhage and Sorensen, 2008). The crucial protein involved in $\mathrm{Ca}^{2+-}$-triggered neuronal exocytosis is synaptotagmin $I$, which binds $\mathrm{Ca}^{2+}$ upon an increase in the presynaptic $\mathrm{Ca}^{2+}$ concentration, and promotes fusion probably through its interactions with both SNAREs (Zhou et al., 2015) and acidic phospholipids (Chapman, 2008).

After fusion, the SNARE complex resides on the presynaptic membrane in a so-called cis configuration (SNAREs are in the same membrane). Disassembly and recycling of the SNAREs is achieved through the dissociation of the helical bundle by the $A A A+$ protein $N S F$ and its adaptor protein, the $\alpha$-soluble NSF attachment protein ( $\alpha$-SNAP) (Jahn and Fasshauer, 2012). The SNARE proteins together with other SV components are retrieved back into the nerve terminal in the form of a new vesicle, which can then undergo another round of exocytosis. 


\subsubsection{Endocytosis}

The best-understood pathway for retrieval of SV components after their complete fusion with the plasma membrane is clathrin-mediated endocytosis (CME) (Jung and Haucke, 2007). It is known that following exocytosis, SV components including the transmembrane proteins are sorted to the outer margin of the active zone, called the periactive zone, which in turn clears the release sites at the active zone for subsequent fusion reactions (Haucke et al., 2011). At the periactive zone, a network of proteinprotein and protein-lipid interactions facilitate the formation of a new vesicle. The most abundant adaptor protein is AP2 which is known to recapture SV proteins from the plasma membrane by interacting with the sorting motifs in the cytoplasmic domains of some of these proteins as well as with the membrane lipid phosphatidylinositol 4,5bisphosphate (PIP2) (Dittman and Ryan, 2009; Jung and Haucke, 2007). Other major adaptor proteins are AP180 and stonin 2. AP180 binds synaptobrevin (Saheki and De Camilli, 2012), and stonin 2 interacts with AP2 and the multidomain scaffold EPS15 (epidermal growth factor receptor substrate 15) and serves as a linker between these proteins and synaptotagmin 1 (Maritzen et al., 2010). Clathrin coat which is created around the nascent vesicle in the form of a hexa-pentagonal array of assembled three light and three heavy chains of clathrin molecules, termed triskelia (Jung and Haucke, 2007), had been assumed to be the primary determinant of membrane curvature (Saheki and De Camilli, 2012). However, more recent models suggest that clathrin stabilizes the curvature and cooperates in propagating the curvature induced by other proteins and accessory factors (Itoh and De Camilli, 2006). These proteins include BAR (Bin, amphiphysin, Rvs)-domain proteins like amphiphysin and endophilin (McMahon and Gallop, 2005). The final step of endocytosis is the detaching of the endocytic bud from the plasma membrane. This requires recruitment of the fission machinery in which dynamin plays the central role (Saheki and De Camilli, 2012). Dynamin interacts with some of SH3-containing proteins like endophilin and amphiphysin to further shape the endocytic bud (Dittman and Ryan, 2009), and finally cuts the neck of the newly sculpted vesicle in a GTP-hydrolysis-dependent reaction (Roux et al., 2006). The endocytic vesicle, after being severed form the plasma membrane, undergoes an uncoating process in which the vesicle sheds all the components of the coating machinery. This process involves ATP-dependent dissociation of the clathrin coat by the ATPase Hsc70 and the 
cofactor protein auxilin (Eisenberg and Greene, 2007), and probably also involves synaptojanin, which helps in destabilizing the coat assembly (Dittman and Ryan, 2009).

Although a large body of evidence has established a central role for CME in SV recycling, two other modes of endocytosis have also been proposed, both of which take place within timescales much faster than CME: kiss-and-run and ultrafast endocytosis. Briefly, in the kiss-and-run pathway the formation of a transient and nanometer-sized fusion pore between the SV and the plasma membrane leads to release of intravesicular contents (Wu et al., 2014). Contrary to CME, kiss-and-run occurs at the active zone (Figure 1-1), and since it precludes full integration of SVs into the plasma membrane, the vesicle can retain all of its molecular components for reuse. It is estimated that a complete round of exo-endocytosis in kiss-and-run takes $<1 \mathrm{~s}$ (Alabi and Tsien, 2013).

Ultrafast endocytosis was recently proposed as a new pathway of endocytosis (Watanabe et al., 2013a). Similar to CME, this mode of endocytosis occurs at the periactive zone. However, it is 200-fold faster than CME, and occurs within 50-100 ms at mouse central synapses (Watanabe et al., 2013b). It is proposed that ultrafast endocytosis restores the surface area of the membrane quickly after exocytosis (Watanabe et al., 2013b). 


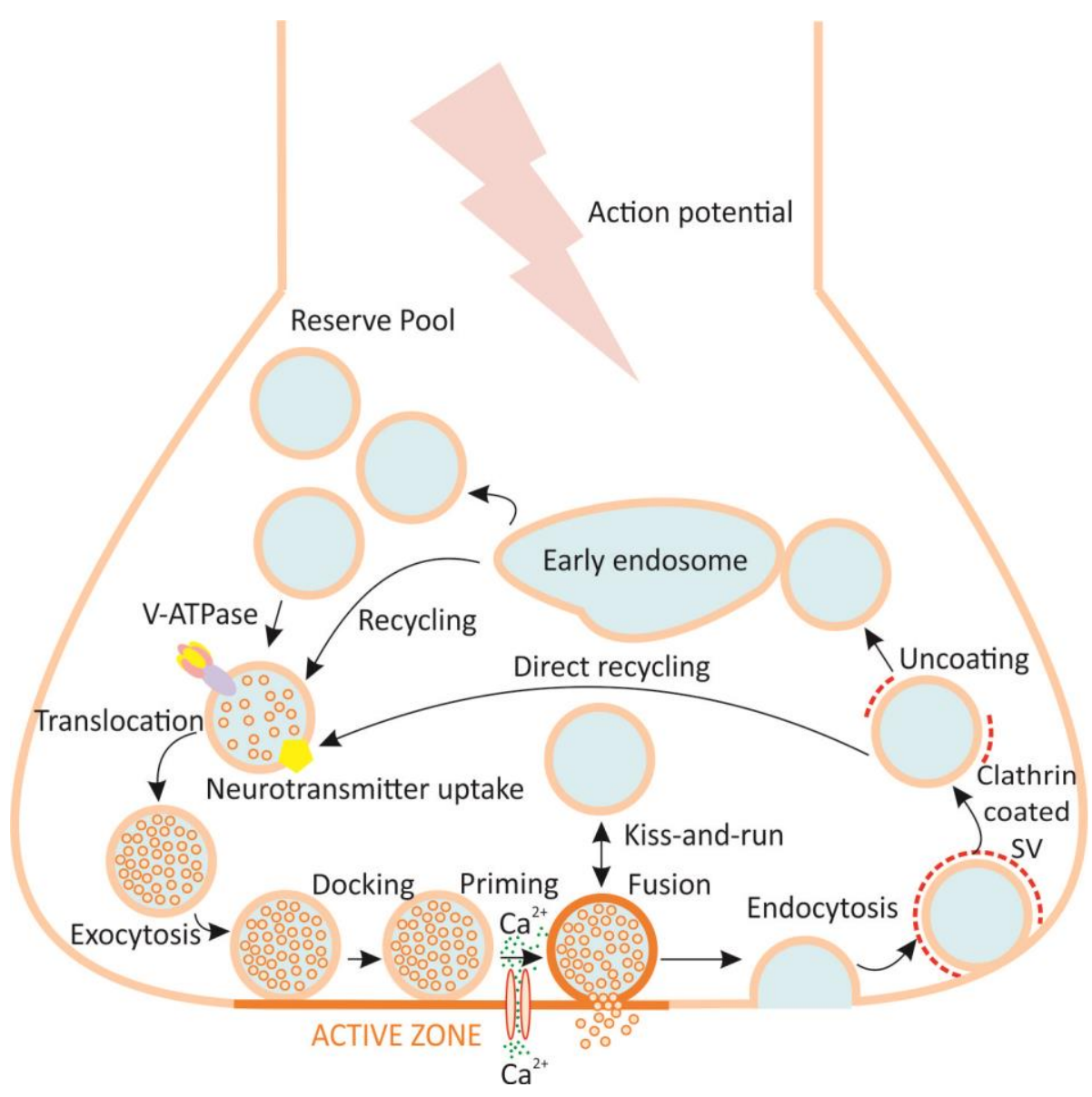

Figure 1-1 Sequence of events at the presynaptic terminal of a chemical synapse.

At chemical synapses, the information is transferred via release of neurotransmitter molecules which are stored in SVs at the presynaptic terminal. The transport of neurotransmitters into the SVs is the result of cooperation between many SV proteins among which the V-ATPase and the vesicular transporters (yellow pentagonal shapes) play the main roles. Filled SVs are translocated to the active zones (dark orange region) where they can undergo docking and priming. Upon arrival of an action potential voltage-sensitive $\mathrm{Ca}^{2+}$ channels open and this triggers fusion of the SVs with the presynaptic membrane and release of their contents. Release occurs via either a transient fusion pore (kiss-and-run) or a full collapse of SVs into the presynaptic membrane. The vesicles can be retrieved back into the nerve terminal via clathrinmediated endocytosis which involves formation of a clathrin coat (dashed red line) around the newly formed SVs. Following uncoating, SVs are regenerated in the nerve terminal, which probably involves trafficking through a presynaptic endosomal compartment. The recycling SVs are in slow exchange with a depot of SVs that constitute the majority of SVs in most presynaptic terminals and are referred to as a reserve pool (adapted from Figure 1 of (Jahn and Fasshauer, 2012)).

\subsection{Neurotransmitter Filling}

As described above, the transfer of information at chemical synapses is mediated by the release of messenger molecules, termed neurotransmitters, from the presynaptic 
neuron. Therefore, SVs have to load more than a thousand neurotransmitter molecules to accomplish neurotransmission regardless of whether they are directly endocytosed form the plasma membrane via CME (Jung and Haucke, 2007), trafficked through a presynaptic endosomal compartment (Wucherpfennig et al., 2003) or recycled via kissand-run (Alabi and Tsien, 2013). The key components of neurotransmitter filling on SVs are the vacuolar proton ATPase (V-ATPase) and the vesicular neurotransmitter transporters. These two proteins mediate two distinct processes; the V-ATPase is in charge of formation of an electrochemical gradient $\left(\Delta \mu_{\mathrm{H}+}\right)$ across the membrane by pumping protons into the lumen of SVs, whereas the transporters are responsible for shuttling the neurotransmitter molecules into the lumen of SVs by utilizing this gradient. The mechanism and regulation of these two processes will be discussed separately in the following sections. However, it should be noted that under physiological conditions where all the prerequisites of vesicle filling such as neurotransmitters and ATP are available, these two processes most likely occur simultaneously.

\subsubsection{Electrochemical Gradient Formation and Regulation}

The V-ATPase is evolutionarily related to F-ATP synthases in the inner mitochondrial membrane and to A-ATP synthases in the plasma membrane of archaea and eubacteria (Marshansky et al., 2014). This large multi-protein complex consists of a peripheral $\mathrm{V}_{1}$ domain, which is responsible for ATP hydrolysis, and an integral $V_{0}$ domain, which operates to translocate the protons across the membrane (Toei et al., 2010). The $V_{1}$ domain contains eight subunits $(A-H)$, among which the $A$ and $B$ subunits are arranged in an alternating fashion forming the catalytic hexamer $\left(\mathrm{A}_{3} \mathrm{~B}_{3}\right)$ of the V-ATPase. The $\mathrm{V}_{0}$ domain is composed of six different subunits (a, c, c', c", d and e), three of which are organized into a ring (Marshansky et al., 2014). ATP hydrolysis at the interface of the A and B subunits of the catalytic hexamer induces a conformational change and provides the free energy required to rotate the ring within the $V_{0}$ domain. Upon one full revolution of the rotor of the V-ATPase, six protons are transported into the lumen of SVs at the expense of three ATP molecules (Grabe et al., 2000). Accumulation of protons in the lumen of SVs not only generates a $\mathrm{pH}$ gradient across the membrane $(\Delta \mathrm{pH})$ but also results in the formation of a membrane potential $(\Delta \psi)$, since no counter ion is transported by the V-ATPase. These two components together are referred to as the electrochemical gradient $\left(\Delta \mu_{\mathrm{H}+}\right)$ or proton motive force (pmf). This $\Delta \mu_{\mathrm{H}_{+}}$opposes the 
action of the proton pump, as the V-ATPase has to overcome the sum of energy resulting from the two components of this gradient in order to accumulate more protons into the lumen of vesicles:

$$
\Delta G_{\text {total }}=\Delta G_{\psi}+\Delta G_{\mathrm{pH}}
$$

where $\Delta G_{\psi}$ and $\Delta G_{\mathrm{pH}}$ are the electrical and chemical energy, respectively, resulting from the proton gradient across the membrane. These energies can be calculated from the following equations:

$$
\begin{aligned}
& \Delta G_{\psi}=z_{\mathrm{H}^{+}} \times F \times \Delta \psi \\
& \Delta G_{p H}=R \times T \times \ln \left(\frac{\left[\mathrm{H}^{+}\right]_{\text {luminal }}}{\left[\mathrm{H}^{+}\right]_{\text {external }}}\right)
\end{aligned}
$$

where $Z_{H^{+}}$is the valence of a proton, $F$ is the Faraday constant, $\Delta \psi$ is the membrane potential, $R$ is the universal gas constant, $T$ is the absolute temperature and $\left[\mathrm{H}^{+}\right]_{\text {luminal }}$ and $\left[\mathrm{H}^{+}\right]_{\text {external }}$ are the concentration of protons in the lumen and in the surrounding environment of the SVs, respectively. By combining Eq. 1.2 and 1.3 with Eq. 1.1, $\Delta \mu_{\mathrm{H}+}$ can be calculated by the following equation:

$$
\Delta \mu_{\mathrm{H}^{+}}=\Delta \psi+\frac{R T}{F} \ln \left(\frac{\left[\mathrm{H}^{+}\right]_{\text {luminal }}}{\left[\mathrm{H}^{+}\right]_{\text {external }}}\right)
$$

While the chemical component is mainly determined by the free luminal and external proton concentrations, the calculation of the electrical component is complicated. As shown in Eq. 1.5, $\Delta \psi$ depends not only on the concentration of free protons, but also on the concentration of buffered protons, other cations and anions as well as on the immobile charged particles (Endresen et al., 2000; Grabe and Oster, 2001; Rybak et al., 1997). It should be noted that this equation gives an estimate of electrostatic membrane potential in the absence of any ion diffusion across the membrane:

$$
\Delta \psi=\frac{F V}{C} \times\left(\sum_{i} \mathrm{z}_{\mathrm{i}}[\text { cations }]_{\mathrm{i}}-\sum_{i} \mathrm{z}_{\mathrm{i}}[\text { anions }]_{\mathrm{i}}+\beta \times \Delta \mathrm{pH}-B\right)
$$


where $F$ is the Faraday constant, $V$ is the volume of the organelle, $C$ is the total capacitance of the membrane, $z$ is the valence of ionic species, $\beta$ is the buffering capacity and $B$ represents fixed negative protein charges trapped in the lumen. The latter parameter $(B)$ sets the zero point of the membrane potential and has been shown to help to maintain an acidic pH in the organelle (Moriyama et al., 1992). According to this equation, all variations in size, shape, protein composition, ion fluxes and luminal buffering capacities of the organelles result in differences in membrane potential and eventually affect $\Delta \mu_{\mathrm{H}+}$.

In addition to all these elements, there are two more attributes, which by affecting the net proton movement across the membrane, influence $\Delta \mu_{\mathrm{H}_{+}}$: proton pumping activity and proton efflux. The proton pumping activity in an organelle is determined by both the activity and the number of active proton pumps. As described above, the eukaryotic VATPase, which is the proton pumping machinery of an organelle, is a multi-subunit membrane protein whose structure and function is largely conserved during evolution (Perez-Sayans et al., 2012). However, it has been shown that V-ATPases in different membrane fractions of the same tissue (Wang and Gluck, 1990) or various tissues (SunWada et al., 2003) differ in their activity and $K_{\mathrm{m}}$ for ATP, due to possessing different isoforms of one or more of the subunits. Moreover, variation in the copy-number of VATPases has been proposed to result in differences in acidification rates in different endocytic vesicles (Van Dyke and Belcher, 1994).

On the other hand, the proton efflux from the lumen affects the extent of the $\mathrm{pH}$ gradient across the membrane. Protons can leave the lumen either via passive leakage through the lipid bilayer or through transmembrane proteins. Passive leakage is affected by the membrane surface area of the organelle and the physical state of the membrane (Wan et al., 2002). Protein-mediated proton efflux can occur through, but not exclusively, all the transporters and ion exchangers of the organelle whose activity involves proton exchange for external molecules.

On the SV, the major proteins, which use the $\mathrm{pH}$ gradient for their activity are the vesicular neurotransmitter transporters. The mechanism of proton coupling with neurotransmitter transport will be further discussed in the following sections. In addition to the neurotransmitter transporters, two main proton exchangers on SVs, the $\mathrm{Na}^{+} / \mathrm{H}^{+}$exchangers (NHE) and the $\mathrm{Cl}^{-} / \mathrm{H}^{+}$exchangers (ClC), also contribute to proton efflux from the lumen. NHEs are conserved transmembrane proton exchangers whose function is vital to the cells and organelles (Bianchini and Poussegur, 1994). While 
NHE1-5 are localized to the plasma membrane in various cells (Orlowski and Grinstein, 2004), NHE6-9 are distributed to the Golgi and endosomal compartments as follows: mid- to trans-Golgi, NHE8; trans-Golgi network, NHE7; early recycling endosomes, NHE6; and late recycling endosomes, NHE9 (Nakamura et al., 2005). NHE6 and 9 have been found on SVs as well (Goh et al., 2011; Gronborg et al., 2010; Preobraschenski et al., 2014). These proton exchangers are proposed to transport extracellular/cytoplasmic $\mathrm{Na}^{+}$or $\mathrm{K}^{+}$at the expense of intracellular/luminal protons with a stoichiometry of $1: 1$ (Bianchini and Poussegur, 1994; Nakamura et al., 2005). However, a recent study on NHE7 has shown that these proton exchangers can transport $\mathrm{Na}^{+}$and $\mathrm{Li}^{+}$but not $\mathrm{K}^{+}$ (Milosavljevic et al., 2014).

In addition to NHEs, ClCs are also of great importance to cells and organelles and have various biological roles, such as regulating skeletal muscle excitability and the facilitation of endosomal acidification (Accardi and Miller, 2004). These transporters include both plasma membrane isoforms (e.g. ClC1 and 2), and intracellular isoforms (ClC3-7) which reside on different organelles including endosomes, lysosomes and SVs (Jentsch, 2008). While the plasma membrane isoforms are known to function as $\mathrm{Cl}^{-}$ channels, the intracellular isoforms are proposed to operate as $\mathrm{Cl}^{-} / \mathrm{H}^{+}$exchangers (Jentsch, 2007). ClC3 and 7 have been detected on purified SVs with mass spectrometry (Gronborg et al., 2010), and probably exchange one luminal protons for two $\mathrm{Cl}^{-}$ions (Hnasko et al., 2010).

All the above-mentioned regulatory elements of $\Delta \mu_{\mathrm{H}+}$ influence neurotransmitter uptake by modulating the driving force. However, the neurotransmitter molecules, themselves, once they are transported into the lumen, also influence $\Delta \mu_{\mathrm{H}+}$. For instance, it is known that glutamate loading into SVs induces higher acidification (Cidon and Sihra, 1989; Maycox et al., 1988). Since glutamate is anionic, this effect is probably due to the dissipation of the membrane potential by glutamate, which allows the V-ATPase to maintain efficient pumping and generate a larger $\Delta \mathrm{pH}$ across the membrane. The enhancement of $\Delta \mathrm{pH}$ is in favor of the vesicles (aminergic, cholinergic and GABAergic), which require a $\mathrm{pH}$ gradient for accumulating their neurotransmitters. This can explain why VGLUTs are also expressed in subpopulations of non-glutamatergic neurons, where their co-localization with other vesicular transporters on SVs leads to enhancement of neurotransmitter loading (Hnasko et al., 2010), a process called 'vesicular synergy' (El Mestikawy et al., 2011). 


\subsubsection{Neurotransmitter Uptake}

Although the loading of all classical neurotransmitters rely on $\Delta \mu_{\mathrm{H}+}$, the contribution of the two components of this gradient, $\Delta \mathrm{pH}$ and $\Delta \psi$, to the uptake of distinct molecular species of neurotransmitters is different and depends on the charge of the molecule. It is known that uptake of positively charged neurotransmitters such as monoamine and acetylcholine depends more on $\Delta \mathrm{pH}$, while the transport of negatively charged glutamate relies mainly on $\Delta \psi$ (Blakely and Edwards, 2012). In the case of the transport of neutral GABA, it is assumed that both components of $\Delta \mu_{\mathrm{H}+}$ play equal roles (AhnertHilger and Jahn, 2011). Other than $\Delta \mu_{\mathrm{H}+}$, there are two important determinants of neurotransmitter loading of SVs: the cytosolic concentration of the neurotransmitters and the characteristics of the vesicular transporters such as their proton/substrate coupling. In the following sections, regulation of the cytosolic concentrations of neurotransmitters and vesicular transporters will be discussed.

\subsubsection{Regulation of the Cytoplasmic Concentrations of Neurotransmitters}

The amount of molecules accumulated in the lumen of SVs is substantially influenced by the concentration of neurotransmitters in the cytoplasm. Two parallel, and in some cases synergistic processes regulate the availability of neurotransmitters at nerve terminals: the neurotransmitter cycle and neurotransmitter biosynthesis. Since this thesis addresses glutamatergic and GABAergic vesicles, from this point on, the focus is on the neurotransmitters glutamate and GABA. In addition, their uptake mechanisms and regulation will primarily be discussed.

There are two classes of plasma membrane neurotransmitter transporters which are involved in recycling of neurotransmitters from the synaptic cleft to the presynaptic terminal: the $\mathrm{Na}^{+} / \mathrm{Cl}^{-}$-dependent neurotransmitter transporters (SCDNTs) and the $\mathrm{Na}^{+} / \mathrm{K}^{+}$-dependent excitatory amino acid transporters (Masson et al., 1999).

All classical neurotransmitters other than glutamate are recycled back into the nerve terminal after SV exocytosis. This is achieved by the activity of the SCDNTs at the nerve terminals (Masson et al., 1999). The SCDNT family encompasses the serotonin transporter, the dopamine transporter, glycine transporters (GLYTs 1a, 1b, 1c, and 2), the norepinephrine transporter (NET), $\Upsilon$-aminobutyric acid (GABA) transporters (GATs 1-4), the proline transporter and the taurine transporter (Mortensen and Amara, 2003). 
Based on immunocytochemical data, some of these transporters are exclusively present in nerve terminals while the others are distributed all over the plasma membrane in neurons and glia cells. For instance, the GABA transporters have five subtypes (GAT1-3, betaine/GABA transporter and rB16a) among which GAT3 is found in glia cells and GAT1 is expressed both in neurons and astrocytes (Masson et al., 1999). The $\mathrm{Na}^{+}$ electrochemical gradient, which is created and maintained by the plasma membrane $\mathrm{Na}^{+} / \mathrm{K}^{+}$-ATPase, is the main driving force utilized by all SCDNTs to transport the extracellular neurotransmitters back to the nerve terminals. They also require $\mathrm{Cl}^{-}$for transport of their substrate, even though the energy derived from the $\mathrm{Cl}^{-}$electrochemical gradient is much lower than that of $\mathrm{Na}^{+}$(Kanner and Schuldiner, 1987).

As mentioned above, the other subfamily of the plasma membrane neurotransmitter transporters is the $\mathrm{Na}^{+} / \mathrm{K}^{+}$-dependent excitatory amino acid transporters, referred to as EAATs (excitatory amino acid transporters), which play a substantial role in the removal of glutamate from the synaptic cleft. These transporters, which share no sequence homology with the SCDNTs, also rely on a $\mathrm{Na}^{+}$electrochemical gradient. They are $\mathrm{Cl}^{-}$ independent, although some may involve $\mathrm{Cl}^{-}$permeation during substrate transport, and they exchange intracellular $\mathrm{K}^{+}$for extracellular glutamate (Amara, 1992). In addition, contrary to presynaptic localized SCDNTs, EAATs are mainly, and in some cases exclusively, expressed in glia cells (Roberts et al., 2014). In case of their neuronal expression (e.g. EAAT-3 and -4), they do not seem to locate to the presynapse, and presumably reside on the postsynaptic membrane (Gundersen et al., 1993; Masson et al., 1999).

Glutamate is not directly recycled back to the nerve terminal but instead undergoes a glutamine-glutamate cycle, in which EAATs play a crucial role. After release from neurons, glutamate is taken up primarily by EAAT-1 and -2 on astrocytes (Masson et al., 1999), and together with ammonia is converted to glutamine by glutamine synthase at the expense of ATP hydrolysis (Curthoys and Watford, 1995). Glutamine is then exported from the astrocytes by system $N$ transporters and retrieved by the neuronal system A neutral amino acid transporters. Transport of glutamine by both system $\mathrm{N}$ and $\mathrm{A}$ transporters is $\mathrm{Na}^{+}$-dependent. However, only in system $\mathrm{N}$ is the transport coupled to $\mathrm{H}^{+}$ translocation, resulting in an electroneutral transport mechanism (Chaudhry et al., 2002). Ultimately, deamination of glutamine by phosphate activated glutaminase (PAG) at the nerve terminals results in glutamate production (Kvamme et al., 2001). Since GABA is derived from glutamate, the glutamate-glutamine cycle contributes to the GABA 
synthesis process as well. It has been shown that inhibition of EAATs in the hippocampus reduces the quantal size at inhibitory synapses (Mathews and Diamond, 2003). Moreover, there is evidence that the blockade of neuronal glutamine transport or inhibition of glutamine synthesis in astrocytes can significantly reduce inhibitory currents, indicating a major contribution of the glutamate-glutamine cycle to inhibitory synaptic strength (Liang et al., 2006).

In addition to neurotransmitter recycling, specific biosynthetic enzymes play substantial roles in regulating the availability of cytosolic neurotransmitters. Among the many enzymes involved in the production of different neurotransmitters, tyrosine hydroxylase, tryptophan hydroxylase, choline acetyltransferase and glutamic acid decarboxylases can be considered as the main biosynthetic enzymes involved in the production of dopamine, serotonin, acetylcholine and GABA, respectively (Blakely and Edwards, 2012). Glutamic acid decarboxylases (GADs), which convert glutamate to GABA, exist as two isoforms, encoded by distinct genes, and are named GAD65 and GAD67 based on their molecular masses ( 65 and $67 \mathrm{kDa}$, respectively). GAD65 is directly associated with SVs through palmitoylation, whereas GAD67 is cytosolic (Blakely and Edwards, 2012). It is reported that palmitoylation of GAD65 is critical for its post-Glogi trafficking to the presynaptic sites. This can explain why GAD65 in contrast to GAD67, which is evenly distributed throughout the cell, is predominantly found in the nerve terminals (Wei and $\mathrm{Wu}, 2008$ ). However, GAD67 seems to be of greater importance to inhibitory neurotransmission. Because GAD67 knock-out mice have significantly reduced GABA contents in their brains and suffer from cleft palate, resulting in neonatal death, whereas lack of GAD65 does not seem to change brain GABA contents or animal behavior (Asada et al., 1997; Condie et al., 1997).

In contrast to most other classical neurotransmitters, glutamate as a neurotransmitter in neurons is mainly produced by the glutamate-glutamine cycle. This is mainly due to lack of pyruvate carboxylase in neurons without which they cannot perform de novo synthesis of glutamate from glucose (Kam and Nicoll, 2007). Net synthesis of glutamate from glucose involves formation of pyruvate molecules via glycolysis, which subsequently enter the tricarboxylic acid (TCA) cycle and form $\alpha$ ketoglutarate, the immediate precursor of glutamate. Pyruvate carboxylase serves to replenish the TCA cycle by mediating the synthesis of the constituents of this cycle. In the absence of this enzyme, neurons therefore depend on production of glutamate by their astrocytic neighbors (Hertz and Zielke, 2004). 


\subsubsection{Vesicular Neurotransmitter Transporters}

In contrast to the above-mentioned plasma membrane neurotransmitter transporters, all of which rely on $\mathrm{Na}^{+}$-electrochemical gradient, the vesicular transporters utilize a $\mathrm{H}^{+}$-electrochemical gradient for accumulation of neurotransmitters in the SV lumen. Based on the protein sequence and substrate specificity, vesicular transporters can be classified into three families (Figure 1-2): the solute carrier family (SLC) 17, SLC18 and SLC32 (Omote and Moriyama, 2013).

SLC17 is a type I phosphate transporter family, which contains nine structurally related proteins and is responsible for the transport of organic anions. SCL17 proteins are integral membrane proteins with 6-12 predicted transmembrane domains. Based on their substrate specificity, they are divided into four distinct subfamilies (Miyaji et al., 2008): i) SLC17A1-4, $\mathrm{Na}^{+}$and inorganic phosphate cotransporters (NPT), are expressed in liver, kidney, muscle, stomach and intestine, and in contrast to the other three subfamilies which are vesicular are located at the plasma membrane (Reimer, 2013); ii) SLC17A5, a lysosomal $\mathrm{H}^{+} /$sialic acid cotransporter or sialin, is ubiquitously expressed in all tissues (Reimer, 2013). This lysosomal protein is also present on SVs, where it is proposed to transport both glutamate and aspartate into the lumen of vesicles. Therefore, it is referred to as vesicular excitatory amino acid transporter (VEAT) (Miyaji et al., 2008). However, whether sialin transports excitatory amino acids (Morland et al., 2013), and even if it does so, whether it contributes to excitatory neurotransmission (Herring et al., 2015) has been challenged recently; iii) SLC17A6-8, vesicular glutamate transporter 1-3 (VGLUT1-3), in contrast to VEATs have a very strict substrate specificity and recognize only glutamate, and not aspartate, as their substrate with a $K_{\mathrm{m}}$ of about 1 $2 \mathrm{mM}$ (Omote and Moriyama, 2013). VGLUTs are mainly expressed in the central nervous system (CNS) but are also found in intrinsic and extrinsic primary afferent neurons of the gut (VGLUT2), $\alpha$ (VGLUT2) and $\beta$ (VGLUT1and 2) cells in pancreatic islets, pinealocytes (VGLUT2), liver and kidney (VGLUT3) (Reimer, 2013). In the brain, VGLUT1 and 2 show a complementary distribution in glutamatergic neurons of cerebral cortical layers, hippocampus, amygdala, thalamus and cerebellum. Moreover, some cholinergic and GABAergic neurons express these two VGLUT isoforms (Reimer and Edwards, 2004). VGLUT3 is expressed in non-glutamatergic neurons such as inhibitory cells in layer II of the parietal cortex, stratum radiatum of CA1-CA3 of the hippocampus, dopaminergic cells in the substantia nigra pars compacta and ventral tegmental area, 
serotonergic cells in the dorsal raphe, cholinergic neurons in the dorsal striatum, as well as astrocytes (Reimer and Edwards, 2004); iv) SLC17A9, vesicular nucleotide transporter (VNUT), plays an essential role in the vesicular storage of ATP in various organelles but predominantly in the brain, adrenal gland and thyroid gland (Sawada et al., 2008). In the brain, it is expressed in the cerebellar cortex, the olfactory bulb and the hippocampus. Immunogold labeling data suggested that a subset of VGLUT1-containing SVs also contain VNUT, while at inhibitory nerve terminals in the hippocampus, VGAT and VNUT are located on distinct vesicles (Larsson et al., 2012).

The SLC18 family is involved in the transport of cationic neurotransmitters and includes vesicular monoamine transporter (VMAT) 1 and 2 (SCL18A1 and SLC18A2, respectively), and the vesicular acetylcholine transporter (VAChT; SLC18A3) (Eiden et al., 2004). VMATs are responsible for transport of serotonin, dopamine, epinephrine and norepinephrine. In addition to these monoamines, VMAT2 transports histamine. VMAT1 is mainly expressed in the adrenal gland, endocrine/paracrine cells of the intestine and stomach. VMAT2 is predominantly expressed in the CNS, but is also found in the peripheral nervous system and enterochromaffin like cells in the stomach (Omote and Moriyama, 2013). In spite of their broad substrate specificity, VMATs have very high affinity for their substrate ( $K_{\mathrm{m}}$ in the micromolar range) in contrast to the low apparent affinity ( $K_{\mathrm{m}}$ in the millimolar range) of almost all the other vesicular neurotransmitter transporters (VGLUTs, VGAT and VAChT) (Blakely and Edwards, 2012). Since the cytosolic concentration of monoamines is very low (Edwards, 2007), the high affinity of VMATs enables them to achieve sufficient transport efficiency. VAChT resembles VMATs in their primary sequences ( $\sim 40 \%$ identity), but transports only acetylcholine with significantly lower affinity compared to VMATs. VAchT is expressed in the brain and intestine (Omote and Moriyama, 2013). All the vesicular amine transporters exchange two luminal protons for one amine substrate molecule. Since the amine substrates are protonated at cytoplasmic $\mathrm{pH}$, their transport involves the movement of only one positive charge for every two protons, resulting in a higher demand of their transporters on $\Delta \mathrm{pH}$ than $\Delta \psi$ (Edwards, 2007).

The sole member of SLC32 is the vesicular GABA transporter (VGAT), which is also referred to as the vesicular inhibitory amino acid transporter (VIAAT), and is expressed in both GABAergic and glycinergic neurons (Ahnert-Hilger et al., 2003; Wojcik et al., 2006). Although it was initially shown that VGAT transports both GABA and glycine with similar affinities (Burger et al., 1991), it was later demonstrated that the affinity of VGAT 
for glycine is considerably lower than its affinity for GABA (McIntire et al., 1997), and that these transmitters compete for vesicular uptake (Wojcik et al., 2006).

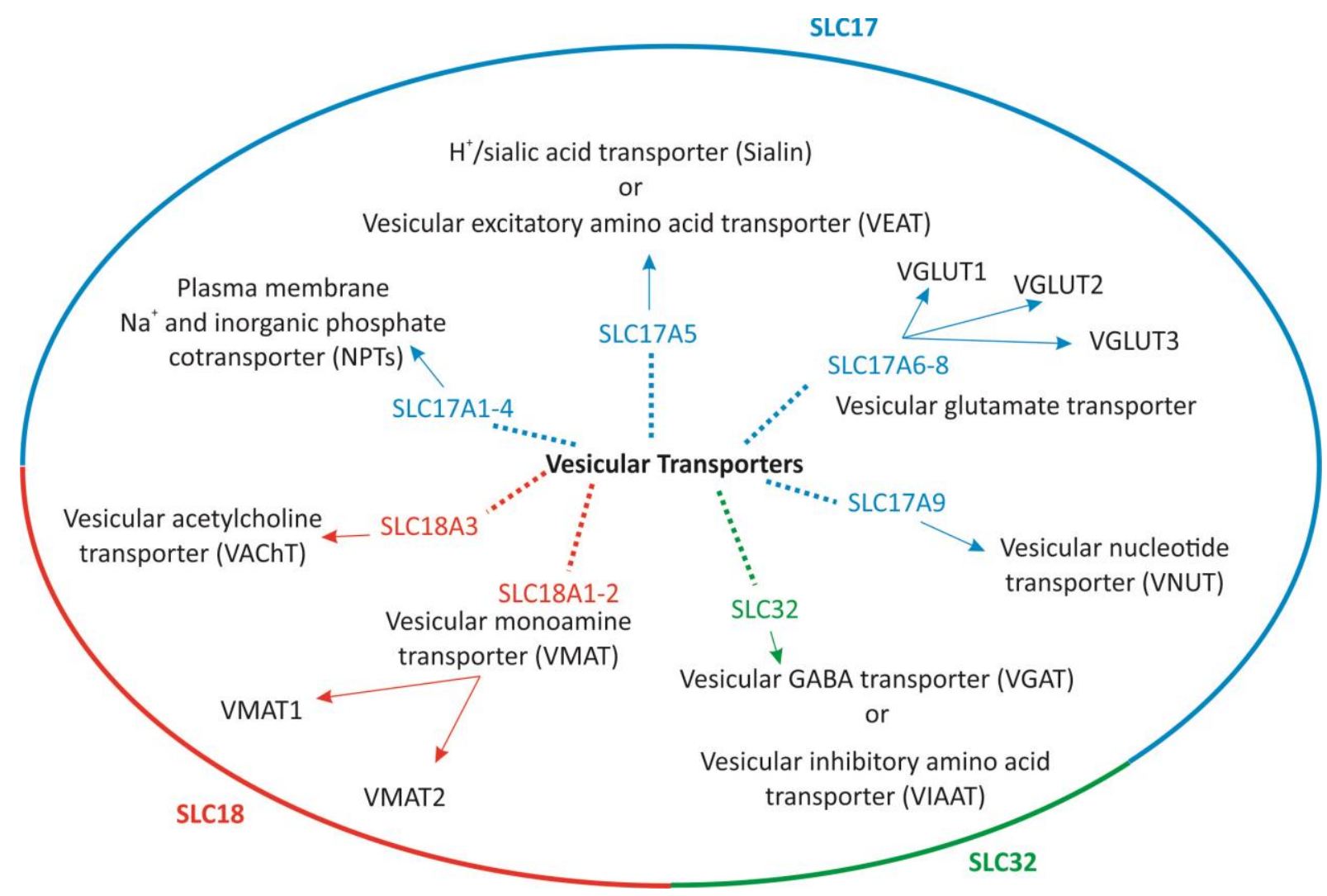

Figure 1-2 Vesicular transporters.

The neurotransmitter transporters on SVs can be divided into three classes based on their substrate specificity and protein sequences (SLC17A1-4 are the only exception to this classification. These proteins are structurally close to the other SLC17 family but reside on the plasma membrane (Reimer, 2013)).

\subsubsection{Transport Mechanism and Regulation of VGLUT and VGAT}

After their discovery, VGLUT1 and VGLUT2 were first characterized as phosphate transporters due to their structural similarity ( 32\%) to NPT1 (Werner et al., 1991), a member of the first subfamily of SLC17 proteins, and were referred to as brain-specific $\mathrm{Na}^{+}$-dependent inorganic phosphate co-transporter I (BNPI) (Ni et al., 1994) and differentiation-associated BNPI (DNPI) (Aihara et al., 2000), respectively. However, later work demonstrated that these transporters localize to glutamatergic neurons and are responsible for glutamate uptake into SVs (Bellocchio, 2000; Fremeau et al., 2001; Takamori et al., 2001; Takamori et al., 2000; Varoqui et al., 2002). There is a general agreement that the transport of glutamate by VGLUTs relies predominantly on $\Delta \psi$, since 
transport is reduced to a great extent by valinomycin, a $\mathrm{K}^{+}$ionophore that dissipates $\Delta \psi$ but not $\Delta$ pH (Bai et al., 2001; Bellocchio, 2000; Fremeau et al., 2002; Fremeau et al., 2001; Gras et al., 2002; Hartinger and Jahn, 1993; Herzog et al., 2001; Schafer et al., 2002; Takamori et al., 2002; Wolosker et al., 1996). Since glutamate is negatively charged at neutral $\mathrm{pH}$, it is estimated that an inside positive $\Delta \psi$ of $\sim 80 \mathrm{mV}$ as the sole driving force for glutamate transport can result in a $\sim 22$ fold higher concentration of glutamate in the lumen of SVs compared to outside (Omote et al., 2011).

The contribution of $\Delta \mathrm{pH}$ to glutamate transport by VGLUT is controversial. While some work has shown that VGLUT functions as a proton exchanger and thus requires $\Delta \mathrm{pH}$ as well as $\Delta \psi$ (Bellocchio, 2000; Carlson et al., 1989; Preobraschenski et al., 2014; Schenck et al., 2009; Wolosker et al., 1996), other studies propose that $\Delta \psi$ is sufficient to provide the required energy for glutamate transport (Juge et al., 2010; Juge et al., 2006; Maycox et al., 1988; Moriyama and Yamamoto, 1995; Omote et al., 2011). Another feature of VGLUT which is essential for its transport is its biphasic dependence on $\mathrm{Cl}^{-}$. It has been shown by many studies that glutamate transport in the absence of $\mathrm{Cl}^{-}$is negligible, reaches its maximal level at $\sim 4 \mathrm{mM} \mathrm{Cl}^{-}$and significantly decreases at higher concentrations of $\mathrm{Cl}^{-}$(e.g. (Bai et al., 2001; Bellocchio, 2000; Hartinger and Jahn, 1993; Juge et al., 2006; Naito and Ueda, 1985; Schafer et al., 2002)). However, how Cl- exerts this effect on VGLUT activity is also controversial. Although most agree that $\mathrm{Cl}^{-}$at low millimolar concentrations acts as an allosteric activator of VGLUT, there is a debate about how $\mathrm{Cl}^{-}$inhibits glutamate transport at higher concentrations. Some believe that it is a secondary effect of $\mathrm{Cl}^{-}$on $\Delta \mu_{\mathrm{H}+}$ which converts $\Delta \psi$ to $\Delta \mathrm{pH}$ and thus reduces the main driving force for glutamate uptake (Hartinger and Jahn, 1993; Juge et al., 2010; Juge et al., 2006). Others have proposed that $\mathrm{Cl}^{-}$not only affects $\Delta \mu_{\mathrm{H}+}$ and dissipates $\Delta \psi$, but also can be transported by VGLUT and thus directly competes with glutamate at high concentrations (Bellocchio, 2000; Schenck et al., 2009).

Recently, a combination of different model systems and approaches provided new insights into the ionic conductances of VGLUT and clarified the above-mentioned controversy to a large extent (Preobraschenski et al., 2014). It was shown that VGLUT indeed transports $\mathrm{Cl}^{-}$, and interestingly also functions as a $\mathrm{K}^{+} / \mathrm{H}^{+}$exchanger. These observations led to a model, according to which VGLUT contains three binding sites: one binding site for monovalent cations which preferentially binds to $\mathrm{K}^{+}$and $\mathrm{H}^{+}$when it is exposed to the cytoplasm and vesicular lumen, respectively, and two anionic binding sites, one of which binds $\mathrm{Cl}^{-}\left(\mathrm{Cl}^{-}\right.$binding site) and the other one preferentially to 
glutamate (glutamate binding site). Based on this model, upon binding glutamate to its binding site, VGLUT undergoes a conformational change from the state where its substrate biding site is exposed to the cytoplasm (state I) to the conformation where the substrate binding pocket is open at the luminal side (state II) (Preobraschenski et al., 2014). This conformational switch is a common mechanistic feature of other members of SLCs as well, such as plasma membrane EAATs and their bacterial/archaeal homologs (GltPh and LeuT), and is referred to as "alternating access": i.e. a substrate/ionic binding site is alternately accessible to the external or internal environment but never to both sides at the same time (Focke et al., 2013). $\mathrm{Cl}^{-}$exerts its stimulatory effect on glutamate loading by accelerating this conformational switch. However, $\mathrm{Cl}^{-}$can also bind to glutamate binding site at high concentrations. Under physiological condition, glutamate binding site of VGLUT is exposed to this high concentration of $\mathrm{Cl}^{-}$when SVs are just endocytosed and engulfed the extracellular fluid which contains more than $100 \mathrm{mM}$ $\mathrm{NaCl}$. Under these circumstances, glutamate binding site of VGLUT is partially occupied by $\mathrm{Cl}^{-}$when VGLUT is in state II. This leads to efflux of $\mathrm{Cl}^{-}$from the lumen of SVs, which may help to maintain the osmotic balance during glutamate loading. Accumulation of glutamate in the lumen together with ongoing V-ATPase activity leads to acidification of the vesicle lumen. This is the condition where $\mathrm{K}^{+} / \mathrm{H}^{+}$antiport comes to play a major role in sustaining glutamate uptake by exchanging the cytoplasmic $\mathrm{K}^{+}$for luminal $\mathrm{H}^{+}$and thus converting $\Delta \mathrm{pH}$ to $\Delta \psi$, which is the main driving force for glutamate uptake. Together, this model explains that VGLUT in addition to its glutamate transport mode, displays a $\mathrm{Cl}^{-}$transport mode and $\mathrm{a} \mathrm{K}^{+} / \mathrm{H}^{+}$antiport mode which are presumably loosely coupled to glutamate transport mode and allow VGLUT to adjust to the changing ionic environment during transport (Preobraschenski et al., 2014).

However, it has been shown that when VGLUT is expressed at the plasma membrane in oocytes, it can also function as a $\mathrm{Na}^{+} / \mathrm{Pi}$ co-transporter (Aihara et al., 2000; Ni et al., 1994). The reconstitution of VGLUT in liposomes suggested that this $\mathrm{Na}^{+}$-dependent phosphate transport mode is distinct from the glutamate transport mode of VGLUT as it is not inhibited by Evans blue, an inhibitor of glutamate uptake, and also does not require $\mathrm{Cl}^{-}$(Juge et al., 2006; Omote et al., 2011). This raises the question of how all these transport activities are carried out by a single protein. Moreover, the details of the different transport modes of VGLUTs and the potential coupling between them are still unknown. 
Regarding the mechanism of GABA transport by VGAT, there are two opposing theories: a GABA/ $\mathrm{H}^{+}$antiport mechanism (Burger et al., 1991; Hell et al., 1990; Maycox et al., 1990; Riazanski et al., 2011) and a GABA/Cl- co-transport mechanism (Juge et al., 2009).

Two observations demonstrated that both $\Delta \psi$ and $\Delta \mathrm{pH}$ play a role in GABA uptake and supported a GABA/ $\mathrm{H}^{+}$antiport mechanism. First, GABA uptake in both isolated SVs and reconstituted liposomes was stimulated by $5-50 \mathrm{mM} \mathrm{Cl}$; whereas at higher concentrations, where $\Delta \psi$ was significantly reduced, a 40-50\% decrease was observed in neurotransmitter uptake. In addition, dissipation of $\Delta \psi$ by $10 \mathrm{mM} \mathrm{SCN}^{-}$reduced GABA uptake, indicating that $\Delta \psi$ is required for efficient GABA loading. By contrast, this concentration of SCN- had no significant effect on uptake of monoamines for which $\Delta \mathrm{pH}$ predominates. Second, dissipation of $\Delta \mathrm{pH}$ with $\mathrm{NH}^{+}$at $\mathrm{pH} 7.3$ completely inhibited GABA uptake while it did not inhibit glutamate uptake by VGLUT but rather enhanced it, suggesting a contribution of $\mathrm{pH}$ gradient to transport of GABA (Burger et al., 1991; Hell et al., 1990).

In contrast with these studies, GABA transport in reconstituted liposomes was only slightly inhibited by $\mathrm{NH}^{+}{ }^{+}$in the recent study where a $\mathrm{GABA} / \mathrm{Cl}^{-}$co-transport mechanism was proposed (Juge et al., 2009). In this study, radiolabeled $\mathrm{Cl}^{-}$uptake by VGATreconstituted liposomes was observed when GABA was present and an inside positive $\Delta \psi$ was formed across the membrane. These data led to the conclusion that $\Delta \psi$ is the main driving force for GABA uptake and that $2 \mathrm{Cl}^{-}$per molecule of GABA are transported by VGAT (Juge et al., 2009). With this contradictory data, the exact mechanism of GABA uptake by VGAT remains enigmatic.

\subsection{Physiological Importance of Neurotransmitter Filling}

The amount of neurotransmitters released by a single SV, referred to as the quantal content, is a basic determinant of synaptic strength. It is evident that both postsynaptic and presynaptic elements can regulate the strength of synaptic transmission. For instance, it is known that the number and properties of postsynaptic receptors can substantially influence synaptic transmission (Sheng and Kim, 2002). At the presynaptic side, vesicle filling affects quantal content and can thereby play a substantial role in regulation of synaptic transmission, particularly when considering that a single quantal release is not sufficient to saturate the post-synaptic receptors (Ishikawa et al., 2002; 
Liu, 2003; Yamashita et al., 2003). Therefore, all the determinants of vesicle filling that were discussed in the previous sections can contribute to the regulation of synaptic transmission. For instance, the activity and abundance of vesicular transporters on SVs can influence synaptic transmission. It has been shown that targeted deletion of VGLUT1 (Wojcik et al., 2004) or VGLUT2 (Moechars et al., 2006) in mice leads to a significant reduction in glutamatergic neurotransmission and causes lethality. On the other hand, overexpression of vesicular transporters resulted in enhanced neurotransmission (Song et al., 1997; Wilson et al., 2005). In fact, the expression level of vesicular transporters is regulated during development (Wojcik et al., 2004) and in response to synaptic activity (Wilson et al., 2005). In addition to vesicular transporters, the cytoplasmic concentration of neurotransmitters can also affect the strength of synaptic transmission. Loading of glutamate via whole-cell recording pipettes into the giant nerve terminal of the brain stem, the calyx of Held, resulted in marked enhancement of glutamate release (Ishikawa et al., 2002).

Beside its primary role as the determinant of quantal content, vesicle filling has also been proposed to influence SV exocytosis. However, this issue is under intense debate. On one side, there are studies which show that impairment of neurotransmitter filling can reduce release probability (Herman et al., 2014) and that overexpression of vesicular transporters can facilitate exocytosis (Song et al., 1997). In conjunction with these findings, it has been shown that the cytosolic concentration of neurotransmitters can regulate the release probability (Wang et al., 2013). On the other hand, other works demonstrate that the probability of vesicle exocytosis is entirely independent of the state of filling of the vesicle, and that empty or semi-filled SVs can undergo cycles of exoand endocytosis (Bartoletti and Thoreson, 2011; Croft et al., 2005; Parsons et al., 1999; Tabares et al., 2001; Wojcik et al., 2004; Zhou et al., 2000). Thus, the possible relationship between the processes of vesicle filling and vesicle release is still an open issue.

\subsection{How to Study Neurotransmitter Filling}

As mentioned above, neurotransmitter filling can be studied as two different, but related processes: $\Delta \mu_{H_{+}}$formation and neurotransmitter uptake. The main approaches to measure $\Delta \mu_{\mathrm{H}+}$ are fluorescence-based and require $\mathrm{pH}$-sensitive or potentiometric probes. Among the various probes which have been utilized for monitoring acidification 
of different organelles (Han and Burgess, 2010), acridine orange has been the most extensively used for measuring the acidification in isolated SVs or reconstituted liposomes (e.g. (Bellocchio, 2000; Schenck et al., 2009)). However, quantitative measurement of $\Delta \mathrm{pH}$ across the membrane with acridine dyes is very difficult, mainly due to highly nonlinear relationship between quenching of the dye and $\Delta \mathrm{pH}$ (Rottenberg and Moreno-Sanchez, 1993).

Recently, $\mathrm{pH}$ sensitive fluorescent proteins such as a mutated green fluorescent protein (GFP) termed pHluorin (Miesenbock et al., 1998) have been used to measure the pH gradient in SVs. In contrast to acridine dyes, these proteins can quantitatively report absolute $\mathrm{pH}$ values in the SV lumen. Moreover, they can be specifically targeted to SVs in neurons by genetic fusion to SV proteins, which makes their in vivo application feasible. In fact, valuable information about the kinetics of vesicle acidification and recycling have been provided by studies in which pHluorin is used as a probe to monitor the luminal $\mathrm{pH}$ of SVs (e.g. (Budzinski et al., 2011; Li et al., 2005)). However, it should be noted that pHluorin has a limited $\mathrm{pH}$ response range and cannot reliably report $\mathrm{pH}$ changes below pH 6 (Sankaranarayanan et al., 2000). This limitation was recently overcome by using mOrange 2 protein, which has a lower $p K_{\mathrm{a}}$ compared to pHluorin and is thus more fluorescent at acidic pH allowing for better measuring SV acidification in hippocampal cultures (Egashira et al., 2015). Despite extensive studies of the pH gradient of $\Delta \mu_{\mathrm{H}+}$, the electrical gradient is largely neglected due to a lack of an appropriate potentiometric probe. Oxonol dyes have been frequently used in in vitro potentiometric assays with SVs (e.g. (Goh et al., 2011; Moriyama et al., 1990; Preobraschenski et al., 2014; Wolosker et al., 1996)). However, the precision of estimates of $\Delta \psi$ using oxonol dyes is quite low (Shapiro, 2000).

In contrast to fluorescence-based acidification and potentiometric assays, common approaches to study neurotransmitter uptake involve either radiolabeled substrates (e.g. (Juge et al., 2006; Maycox et al., 1988)), which is limited to isolated SVs or reconstituted liposomes, or electrophysiology in which the post-synaptic response provides an indirect readout for the amount of loaded neurotransmitters (e.g. (Goh et al., 2011; Riazanski et al., 2011)). However, recent advances in fluorescent probes have introduced novel approaches for directly investigating SV filling (Hires et al., 2008; Masharina et al., 2012; Okumoto et al., 2005). For instance, a new GFP-based glutamate sensor was recently used to measure glutamate release from neurons (Marvin et al., 2013). Similar to pHluorin-based measurements, such a probe can be targeted to the 
lumen of SVs to directly monitor vesicle filling. However, a drawback to such probes is that they usually have low $K_{\mathrm{d}}$ (in micromolar range) (Hires et al., 2008) and are saturated at relatively low substrate concentrations, while the luminal concentration of neurotransmitters might exceed 100 mM (Hori and Takahashi, 2012). Thus, mutational screening to find sensors with a sufficiently low sensitivity to measure the high luminal concentration of neurotransmitters would be of great interest.

\subsection{Aim of this Study}

It has been estimated that a single SV loads more than 2,000 neurotransmitter molecules (Hori and Takahashi, 2012) within the few seconds of SV recycling (Qiu et al., 2015; Sara et al., 2002). As described above, vesicle filling is a complex process and requires generation of an electrochemical proton gradient to fuel the vesicular transporters. Moreover, the relative contribution of $\Delta \mathrm{pH}$ and $\Delta \psi$ to the uptake of distinct neurotransmitters is different and depends on the charge of the neurotransmitter molecules (Edwards, 2007). Therefore, additional mechanisms which tailor $\Delta \mu_{\mathrm{H}+}$ to requirements of vesicular transporters would be beneficial for loading.

In this thesis, I aimed to test whether such mechanisms exist in SVs, and if this were the case, whether SVs with distinct bioenergetic requirements would differ in their regulatory mechanisms. To resolve these issues, accurate measurement of the two components of $\Delta \mu_{\mathrm{H}+}$ and their comparison in vesicles with different transport characteristics was required. However, the conventional acidification or potentiometric bulk assays, in which a suspension of isolated SVs are measured, provides averaged information over a heterogeneous vesicle population and do not allow to distinguish different populations of SVs.

Although these approaches have provided valuable information about the mechanism and regulation of $\Delta \mu_{\mathrm{H}+}$ in SVs, they inevitably report the response of dominating subpopulations within the sample, which might mask the response of other vesicle populations. For instance, in a study where the effect of a loss of ClC3 on SV acidification was investigated, no difference in $\mathrm{Cl}^{-}$-dependent acidification of SVs isolated from brains of ClC3 knockout mice was observed compared to wild type (Schenck et al., 2009). However, when GABAergic SVs, whose response was masked by the glutamatergic SVs in the previous experiments, were immune-isolated from the SV population, a significantly impaired $\mathrm{Cl}^{-}$-dependent acidification was measured (Riazanski et al., 2011). 
In order to avoid this problem and directly compare the potential differences between different SV populations, I optimized a microscopy-based single-vesicle assay to be able to track the response of single vesicles individually. Since SVs are only $\sim 40$ $\mathrm{nm}$ in diameter (Takamori et al., 2006), such an assay demanded appropriate probes which could report the response of single SVs with a sufficiently high signal-to-noise ratio. Upon testing several $\mathrm{pH}$-sensitive and potentiometric probes, I found superecliptic pHluorin and VF2.1.Cl suitable for the quantitative measurement of $\mathrm{pH}$ and membrane potential of single vesicles, respectively. These probes were able to provide adequate signal at the single vesicle level and possessed sufficiently high photostability.

Therefore, for acidification measurements, I purified SVs (spH-SVs) from transgenic mice expressing super-ecliptic pHluorin fused to the C-terminus of VAMP2 (Budzinski et al., 2011; Li et al., 2005), and for potentiometric measurements I purified SVs from wild type mouse brain and labeled them with VF2.1.Cl. I used a TIRF-microscopy setup equipped with a solution exchange system and a UV-flash lamp for measuring the response of immobilized SVs. Moreover, I employed this assay to compare the response of the major excitatory (glutamatergic) and inhibitory (GABAergic) SV populations, whose reliance on the two components of $\Delta \mu_{\mathrm{H}+}$ is different, to test whether vesicles with different transport characteristics differ in their regulation of $\Delta \mu_{\mathrm{H}+}$. For this purpose, I designed on-stage antibody staining experiments in which I labeled SVs with antibodies against VGLUT1 or VGAT to unequivocally distinguish GABAergic from glutamatergic SVs after each experiment.

With this assay, I was able to present a comprehensive picture of how $\Delta \mu_{H_{+}}$is regulated and adjusted to the requirements of different synaptic vesicle populations. 


\section{Materials and Methods}

\subsection{Buffers}

Table 2-1 Solutions and Buffers

\begin{tabular}{|c|c|c|}
\hline Solution & Components & Concentration (mM) \\
\hline \multirow{3}{*}{ Glycine buffer } & Glycine & 300 \\
\hline & MOPS (pH 7.4) & 10 \\
\hline & $\mathrm{MgSO}_{4}$ & 4 \\
\hline \multirow{3}{*}{ K-gluconate buffer } & K-gluconate & 100 \\
\hline & MOPS (pH 7.4) & 10 \\
\hline & $\mathrm{MgSO}_{4}$ & 4 \\
\hline \multirow{2}{*}{ Homogenization buffer } & Sucrose & 320 \\
\hline & HEPES (pH 7.4) & 5 \\
\hline \multirow{2}{*}{ Sucrose Cushion } & Sucrose & 700 \\
\hline & HEPES (pH 7.4) & 10 \\
\hline \multirow{2}{*}{ Chromatography column buffer } & Glycine & 300 \\
\hline & HEPES (pH 7.4) & 5 \\
\hline \multirow{2}{*}{ Phosphate buffer saline (PBS) } & $\mathrm{NaCl}$ & 150 \\
\hline & $\mathrm{Na}_{2} \mathrm{HPO}_{4}(\mathrm{pH} 7.4)$ & 20 \\
\hline \multirow{7}{*}{ Sodium buffer } & $\mathrm{NaCl}$ & 140 \\
\hline & $\mathrm{KCl}$ & 5 \\
\hline & Glucose & 10 \\
\hline & $\mathrm{NaHCO}_{3}$ & 5 \\
\hline & $\mathrm{Na}_{2} \mathrm{HPO}_{4}$ & 1.2 \\
\hline & $\mathrm{MgCl}_{2}$ & 1 \\
\hline & HEPES (pH 7.4) & 20 \\
\hline
\end{tabular}

\subsection{Imaging Setup}

Table 2-2 Microscope and Other Equipment of Imaging Setup

\begin{tabular}{|c|c|c|}
\hline Equipment & Characteristics & Manufacturer \\
\hline Glass coverslips & $\begin{array}{c}18 \mathrm{~mm} \emptyset \\
\text { Thickness No. } 1.5 \mathrm{H}(0.170 \mathrm{~mm} \pm \\
0.005 \mathrm{~mm})\end{array}$ & $\begin{array}{l}\text { Paul Marienfeld GmbH \& } \\
\text { Co.KG }\end{array}$ \\
\hline Axiovert 200 & $\begin{array}{l}\text { Inverted fluorescence/phase or } \\
\text { DIC microscope }\end{array}$ & Carl Zeiss \\
\hline EM-CCD camera & iXON3 897 & Andor Technology \\
\hline Argon gas laser & $\begin{array}{l}\text { multi-line argon gas laser of } 800 \\
\text { series } \\
488-514 \mathrm{~nm} \text { laser wavelengths }\end{array}$ & National Laser Company \\
\hline Diode laser & $\begin{array}{l}641 \mathrm{~nm} \text { wavelength } \\
100 \mathrm{~mW} \text { max. power }\end{array}$ & Coherent Deutschland GmbH \\
\hline Optical fiber & multi-wavelength operation & Point Source Ltd. \\
\hline
\end{tabular}




\begin{tabular}{|c|c|c|}
\hline & $\begin{array}{c}(488,568,647 \mathrm{~nm}) \\
100 \mathrm{~mW} \text { max. input power }\end{array}$ & \\
\hline Solenoid shutters & $\begin{array}{l}82 \times 50.8 \times 33.8 \mathrm{~mm} \\
<1.5 \mathrm{msec} \text { activation } \\
\text { opening/closing time }\end{array}$ & Thorlabs GmbH \\
\hline M1-M6 mirrors & $\begin{array}{l}25.4 \text { mm diameter broadband } \\
\text { dielectric mirrors }\end{array}$ & Thorlabs GmbH \\
\hline Dichroic beamsplitter & $\begin{array}{c}98 \% \text { reflection of } 473-491 \mathrm{~nm} \\
95 \% \text { Transmission of } 514-647 \\
n m\end{array}$ & AHF analysentechnik AG \\
\hline Bandpass filter & $\begin{array}{l}488 / 10 \mathrm{~nm} \\
515 / 30 \mathrm{~nm}\end{array}$ & AHF analysentechnik AG \\
\hline Longpass beamsplitter & $\begin{array}{l}\text { F48-425 } \\
\text { T } 425 \text { LPXR }\end{array}$ & AHF analysentechnik AG \\
\hline Dual line beamsplitter & $\begin{array}{c}\text { ZT488/640rpc } \\
\text { Reflection at } 480-494 \mathrm{~nm} \text { and } \\
633-647 \mathrm{~nm}\end{array}$ & Chroma Technology Corp \\
\hline Dual band emitter filter & $\begin{array}{c}\text { Transmits } 510-563 \text { and } 660-738 \\
\mathrm{~nm}\end{array}$ & AHF analysentechnik AG \\
\hline Lenses & $\begin{array}{c}25 \mathrm{~mm} \varnothing \\
65,75 \text { and } 85 \mathrm{~mm} \text { focal length }\end{array}$ & Melles Griot BV \\
\hline Objective & $\begin{array}{c}\text { PLAN-FLUAR } \\
\text { 100x 1.45 NA, oil immersion }\end{array}$ & Carl Zeiss \\
\hline UV-flash lamp & JML-C2- ms Pulse & Rapp OptoElectronic GmbH \\
\hline Valve system & VC-6 PTFE & Warner Instruments \\
\hline Peristaltic pump & $\begin{array}{c}\text { MINIPULSE } 3 \\
\text { Max. flow rate } 30 \mathrm{~mL} / \mathrm{min}\end{array}$ & Gilson \\
\hline
\end{tabular}

\subsection{Reagents}

Table 2-3 Fluorescent Probes

\begin{tabular}{|c|c|c|}
\hline Indicator & Catalog number & Supplier \\
\hline LysosensorTM Green DND-189 & L-7537 & Molecular Probes \\
\hline LysosensorTM Blue DND-167 & L-7533 & Molecular Probes \\
\hline LysoTracker Green DND-26 & L-7526 & Molecular Probes \\
\hline LysoTracker Red DND-99 & L-7528 & Molecular Probes \\
\hline Acridine Orange & A1301 & Molecular Probes \\
\hline ACMA $^{1}$ & A1324 & Molecular Probes \\
\hline HPTS2 (Pyranine) & H-348 & Molecular Probes \\
\hline Fluo-4 & F-14201 & Molecular Probes \\
\hline SNARF $5 F$ & S-23922 & Molecular Probes \\
\hline $\mathrm{DiD}^{3}$ & D-7757 & Molecular Probes \\
\hline Di-8-ANEPPS & D-3167 & Molecular Probes \\
\hline Oxonal VI & 0267 & Molecular Probes \\
\hline VF2.1.Cl & - & $\begin{array}{l}\text { Max-Planck institute for Biophysical } \\
\text { Chemistry }\end{array}$ \\
\hline FM 1-43 & $\mathrm{T}-35356$ & Molecular Probes \\
\hline
\end{tabular}

19-Amino-6-Chloro-2-Methoxyacridine

2 8-Hydroxypyrene-1,3,6-Trisulfonic Acid

3 1,1'-Dioctadecyl-3,3,3',3'-Tetramethylindodicarbocyanine 
Table 2-4 List of Other Reagents

\begin{tabular}{|c|c|c|}
\hline Material & Catalog number & Company \\
\hline Hellmanex-II & RV 800237 & Hellma Analytics \\
\hline Poly-L-Lysine & P-8920 & Sigma - Aldrich \\
\hline FluoSpheres, $0.2 \mu \mathrm{m}$, yellow-green & F-8811 & Molecular Probes \\
\hline FCCP1 & C2759 & Sigma - Aldrich \\
\hline Bafilomycin A1 & 196000 & Calbiochem \\
\hline Bovine serum albumin (BSA) & A3059 & Sigma - Aldrich \\
\hline $\mathrm{PMSF}^{2}$ & A0999 & AppliChem \\
\hline Pepstatin A & P4265 & Sigma - Aldrich \\
\hline $\mathrm{DPX}^{3}$ & $X-1525$ & Molecular Probes \\
\hline EIPA $^{4}$ & A3085 & Sigma - Aldrich \\
\hline $\mathrm{NPE}^{5}-\mathrm{ATP}^{6}$ & NU-301 & Jena Bioscience \\
\hline Mg-ATP6 & A9187 & Sigma - Aldrich \\
\hline
\end{tabular}

${ }^{1}$ Carbonyl cyanide-p-trifluoromethoxyphenylhydrazone

2 Phenylmethylsulfonyl fluoride

${ }^{3} p$-Xylene-Bis-Pyridinium Bromide

4 -(N-Ethyl-N-isopropyl)amiloride

${ }^{5} \mathrm{P}^{3}$-(1-(2-nitrophenyl)-ethyl)-ester

${ }^{6} \mathrm{pH}$ was set to 7.4

\subsection{Antibodies}

Table 2-5 Antibodies

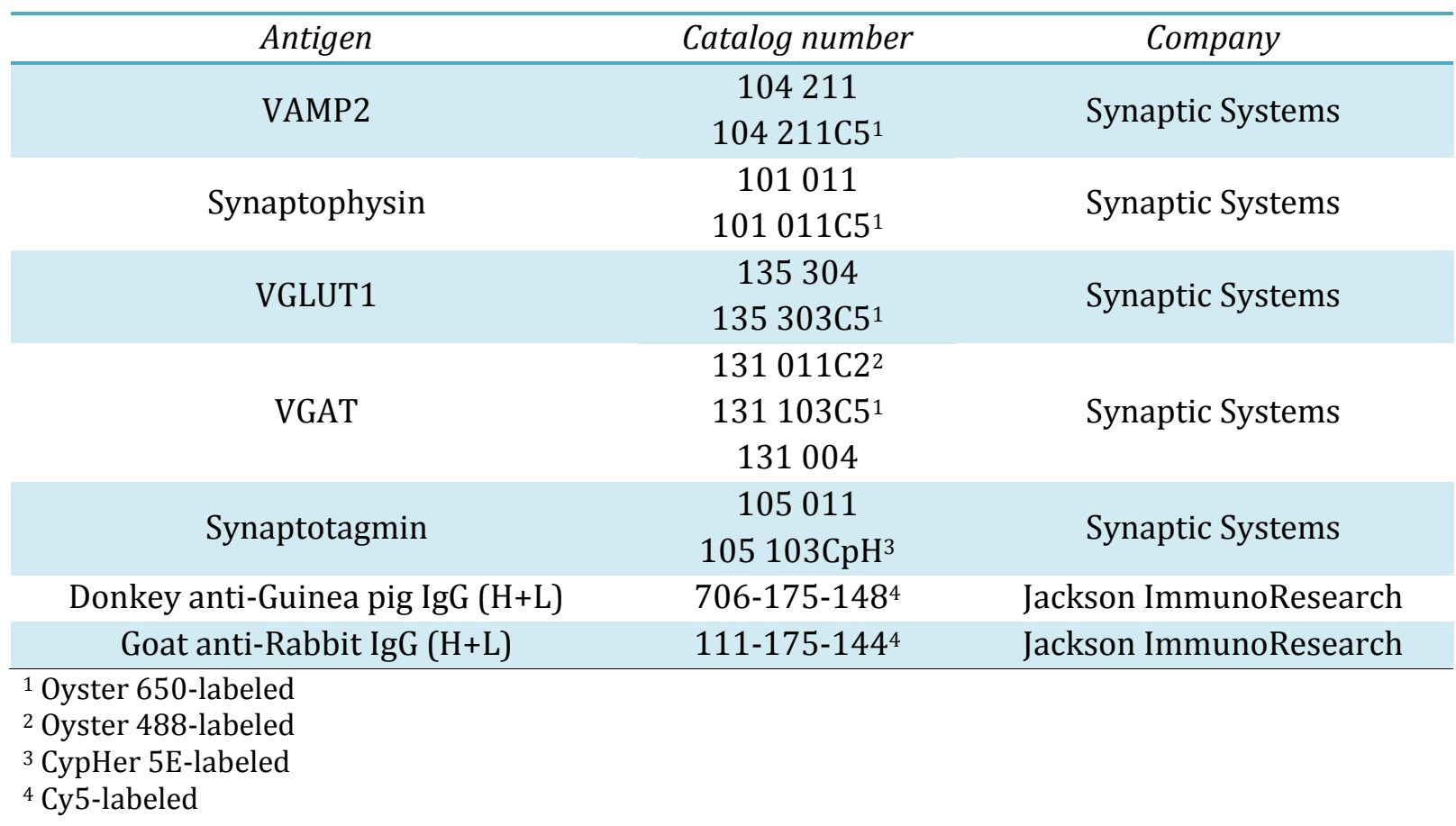

\subsection{Instruments}

Table 2-6 List of Other Instruments

\begin{tabular}{cc}
\hline Equipment & Manufacturer \\
\hline Fluorolog III spectrofluorometer (Model FL322) & Jobin Yvon \\
\hline
\end{tabular}




\subsection{Developing a Single Vesicle Assay}

\subsubsection{Isolation of Synaptic Vesicles}

To purify SVs from rat brain, a previously described protocol was followed (Huttner et al., 1983; Nagy et al., 1976) (Figure 2-1A). Briefly, 20 rats were decapitated and their whole brains were homogenized in ice-cold homogenization buffer (Table 2-1) supplemented with $200 \mu \mathrm{M}$ PMSF and $1 \mu \mathrm{g} / \mathrm{ml}$ Pepstatin A. Synaptosomes (isolated nerve terminals) were then obtained from the homogenate by two steps of differential centrifugations, after which they were washed and hypo-osmotically disrupted. Crude synaptic vesicles (LP2) derived from synaptosomes were loaded on a continuous sucrose gradient and centrifuged at $82,500 \mathrm{x} g$ for 4 hours. The SVs were then collected from the appropriate zone on the gradient and loaded on a controlled-pore glass bead (CPG-3000) column. In the elution chromatogram two peaks were observed. The fractions from the second peak, which was shown to contain synaptic vesicles (Huttner et al., 1983; Nagy et al., 1976), were pooled (Figure 2-1B).

A

$$
\text { Homogenate }
$$

H

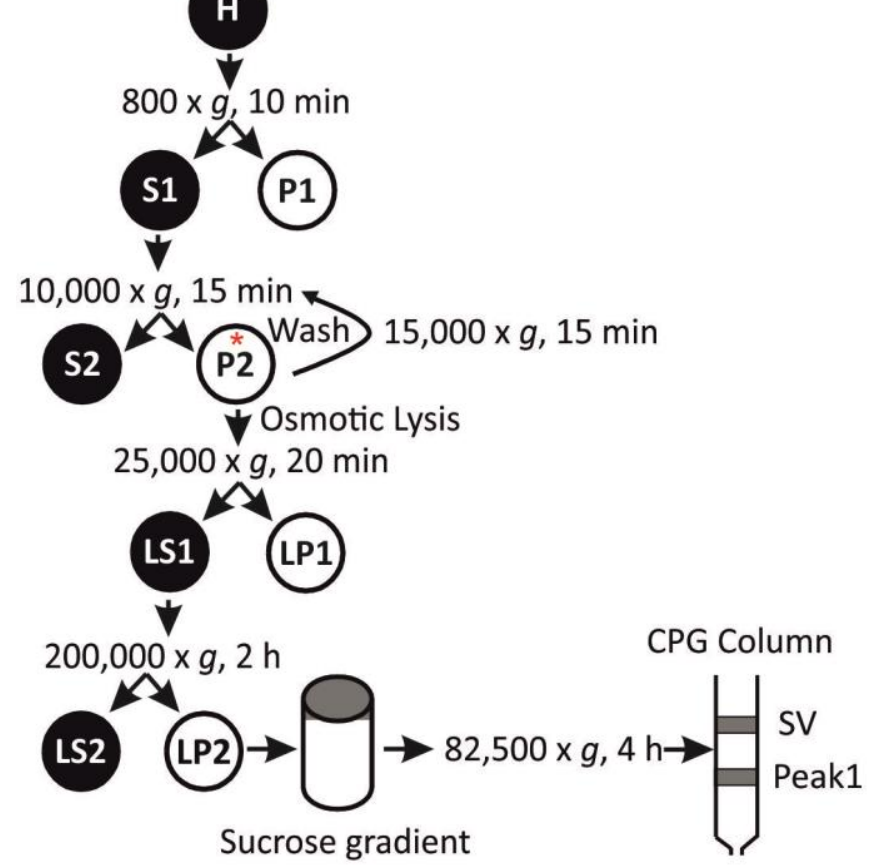

* Synaptosomes

\section{B}

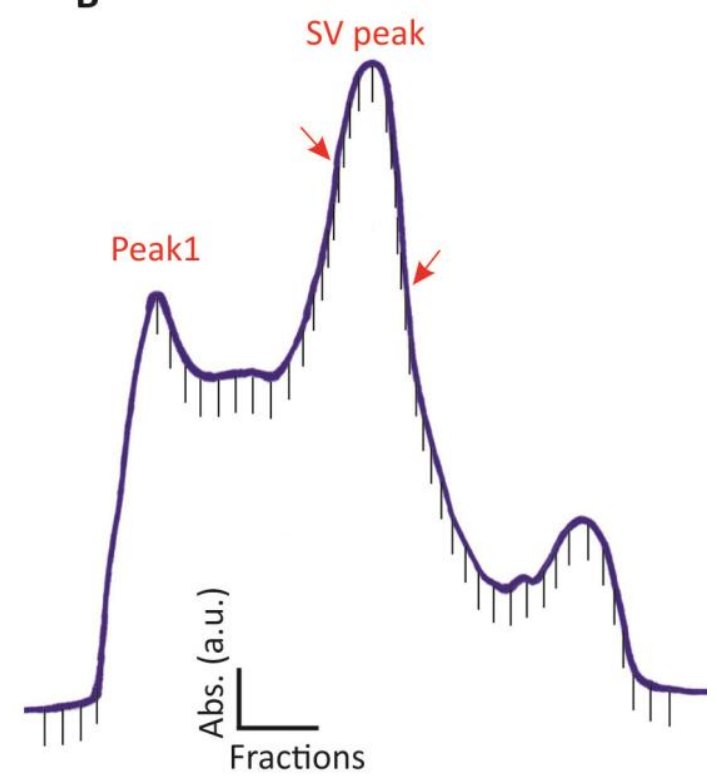

Figure 2-1 Purification of SVs from rat brains.

A) A flow diagram of purification of SVs from rat brains according to the protocol from (Huttner et al., 1983; Nagy et al., 1976). B) A representative elution profile obtained after loading the SV sample on a CPG column. Each line below the chromatogram represents one fraction. While 
'Peak1' contained larger membrane structures, homogenous small SVs were found in 'SV peak'. Red arrows indicate the collected SV fractions.

To purify SVs from wild type and transgenic mouse brains, a recently published protocol (Ahmed et al., 2013) was used. Similar to the above-mentioned protocol, this protocol also included isolation and osmotic lysis of synaptosomes, rate-zonal sucrose gradient centrifugation and size exclusion chromatography. However, as depicted in Figure 2-2, three steps of the protocol were modified for the purpose of this thesis: 1) Synaptosomes were washed once more before osmotic lysis to decrease mitochondrial contamination. 2) The S2 fraction was discarded and LS1 was layered on the sucrose cushion (Table 2-1). As shown in Figure 2-2C, the migration pattern of SVs into the cushion was the same as in the published protocol (compare with Figure 2b of (Ahmed et al., 2013)). Removal of the S2 fraction helped for better separation of the SV peak from the first peak after size exclusion chromatography. This in turn increased the purity of final SV fraction (verified by negative staining EM in collaboration with Dr. Dietmar Reidel, Laboratory of electron microscopy, MPIbpc, Göttingen, Germany). However, to obtain the same yield, the number of mice was doubled. 3) To decrease ionic contamination of the final SV fraction, a $100 \mathrm{~cm} \times 1 \mathrm{~cm}$ column was packed with CPG-3000 beads for the last step of purification. Contrary to the Sephacryl S-1000 column, which requires a high-salt column buffer $(100 \mathrm{mM}$ Tris- $\mathrm{HCl}, 100 \mathrm{mM} \mathrm{KCl}, \mathrm{pH}$ 7.6), the CPG-column allowed for elution of vesicles with a glycine-based column buffer, which is free of membrane-permeable ions (Table 2-1). 

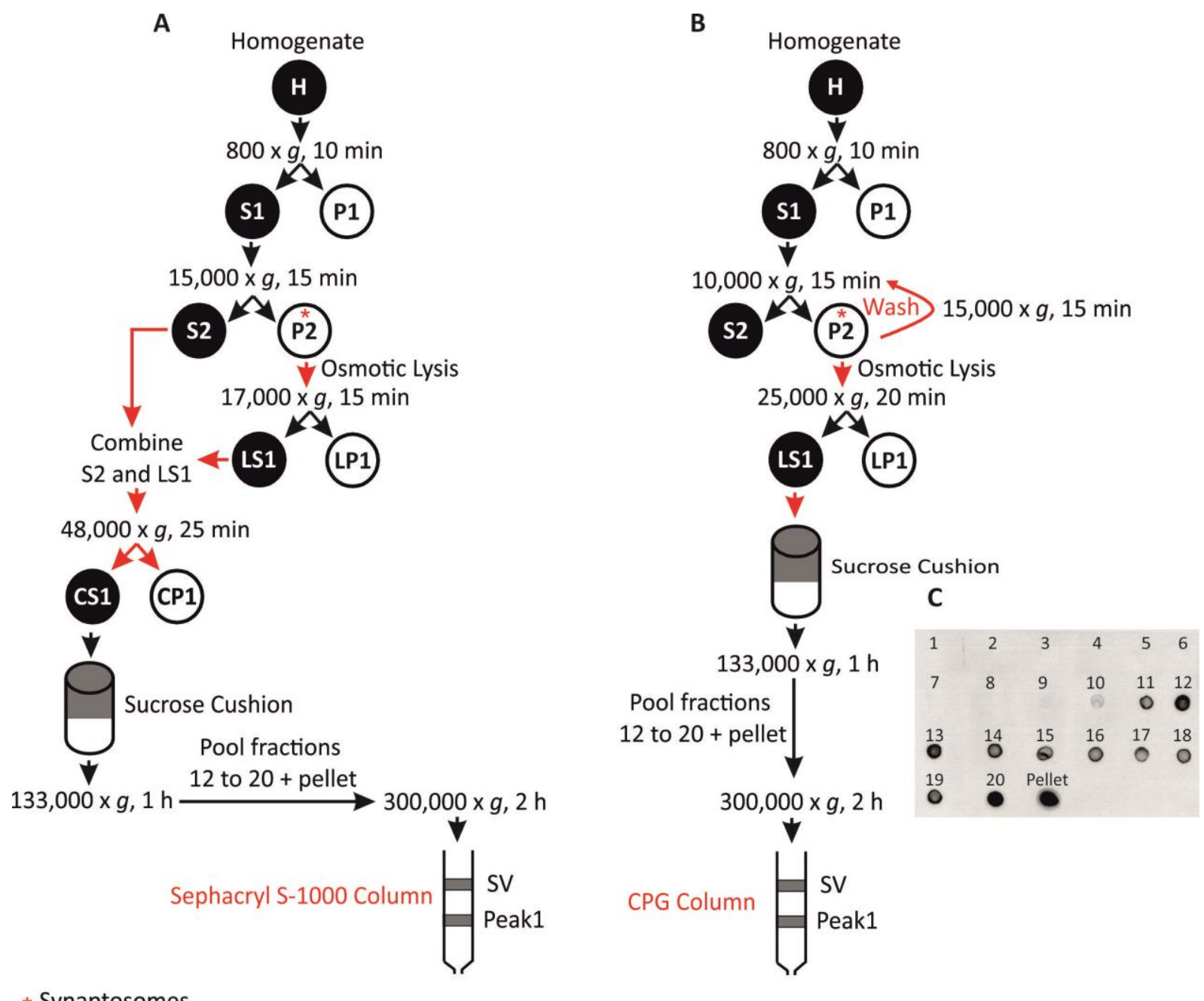

* Synaptosomes

Figure 2-2 Optimization of the purification protocol for purifying SVs from mouse brain.

A) Diagram of the SV purification protocol adapted from (Ahmed et al., 2013). B) Diagram of modified protocol used in this thesis. The steps which were modified are marked with red arrows in each diagram. C) After centrifugation, the sucrose cushion was fractionated into 20 fractions. From each fraction $3 \mu \mathrm{l}$ was spotted onto a nitrocellulose membrane and blotted with antibody against synaptophysin 1 (Appendix 2). The same migration pattern as reported by (Ahmed et al., 2013) was obtained. Fractions 12 to 20 were pooled for further purification.

\subsubsection{Immobilization of Synaptic Vesicles}

Glass coverslips (Table 2-2) were thoroughly cleaned by bath sonication for $20 \mathrm{~min}$ in 2\% (v/v) Hellmanex-II solution (Table 2-4), followed by 20 min bath sonication in MilliQ-purified water. The coverslips were kept in 70\% ethanol until use. Before SV immobilization, the coverslips were rinsed with Milli-Q-purified water, mounted in custom-designed imaging chambers and coated with $0.1 \%(\mathrm{w} / \mathrm{v})$ poly-L-lysine (PLL, Table 2-4) for $20 \mathrm{~min}$. The PLL was then collected from the glass surface and coverslips 
were washed 3 times with Milli-Q-purified water. Next, the protein concentration of the final SV fraction after elution from chromatography column was determined using Pierce BCA Protein Assay Kits (ThermoFisher) according to the manufacturer's manual. 50-100 ng of purified SVs were incubated on PLL-coated coverslips for 1 hour at $4^{\circ} \mathrm{C}$. Non-adsorbed SVs were removed from coverslips before imaging.

\subsubsection{Imaging of Synaptic Vesicles}

\subsubsection{Optical Alignment in TIRF Setup}

For imaging of immobilized SVs, a home-built total-internal reflection fluorescence microscope (TIRFM) was used, based on an Axiovert 200 microscope and a backilluminated EM-CCD camera. This setup had a multi-line argon gas laser which provides multiple laser lines in the range between $488-514 \mathrm{~nm}$ wavelength at a $225 \mathrm{~mW}$ maximum power, and a diode laser which provides a $641 \mathrm{~nm}$ wavelength at $100 \mathrm{~mW}$ maximum power. A 488/10 nm filter was placed in the argon laser path to select the 488 nm laser line. As depicted in Figure 2-3, both laser lines were guided to a two-meter optical fiber through two solenoid shutters, six high surface quality mirrors (M1-M6) as well as a dichroic beamsplitter (Table 2-2). The laser beam from the fiber was deviated by a right angle prism by $90^{\circ}$ and directed to a filterset cube on the reflector turret of the microscope through two achromatic doublet lenses. These lenses, with focal length of 75 and $65 \mathrm{~mm}$, focused the lasers at the back-focal-plane of a PLAN-FLUAR 100x 1.45 NA objective. In addition, a micromanipulator was coupled to the fiber-prism holder to control the horizontal and vertical movement of the prism. A total-internal reflection angle was achieved by de-centering the laser beam using this micromanipulator. Moreover, a dual line beamsplitter (ZT488/640rpc) was placed in the filter cube to be able to illuminate the sample with both lasers and UV light through the objective. No excitation filter was used in the filter cube, and either a dual band 538/685 filter or a $515 / 30 \mathrm{~nm}$ bandpass filter was used as the emission filter. Images were acquired using Andor IQ2 software (Andor Technology) which offers tight synchronization of the EMCCD camera with external events such as opening of the laser shutters via TTL triggers. Moreover, this software allows for programing different imaging protocols, in which 
exposure time, imaging frequency, sequence of external events, etc. can be set according to the purpose of the experiment.

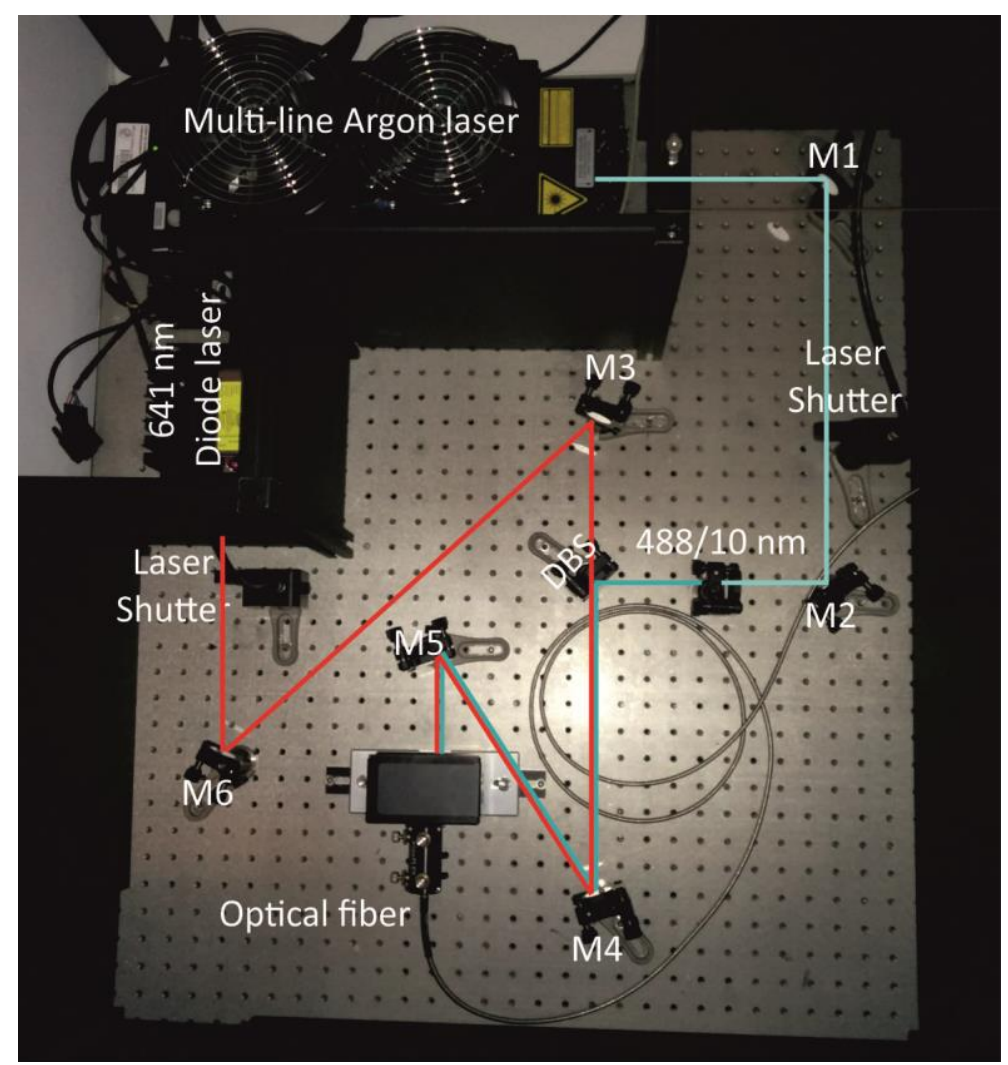

Figure 2-3 Laser alignment in TIRF setup.

The multi-line argon laser and diode laser were guided to an optical fiber via a dichroic beamsplitter (DBS) (LM01-503-25, AHF analysentechnik) and six 25.4 mm-diameter broadband dielectric mirrors (M1-M6). A 488/10 nm filter was placed in the argon laser path to select for the $488 \mathrm{~nm}$ line of the laser.

In order to test the quality of TIRF setup and compare it with epifluorescence, $0.2 \mu \mathrm{m}$ yellow-green fluorescent beads were immobilized on PLL-coated coverslips, and a green fluorescent dye (Pyranine, Exc450 nm/Em511 nm) was added to the bath solution. Beads were illuminated with the $488 \mathrm{~nm}$ line of argon laser and their emission was collected in both TIRF and epifluorescence mode. As shown in Figure 2-4, TIRF excitation could effectively eliminate background fluorescence and in turn improve the signal-to-noise ratio (SNR) compared to epifluorescence. 

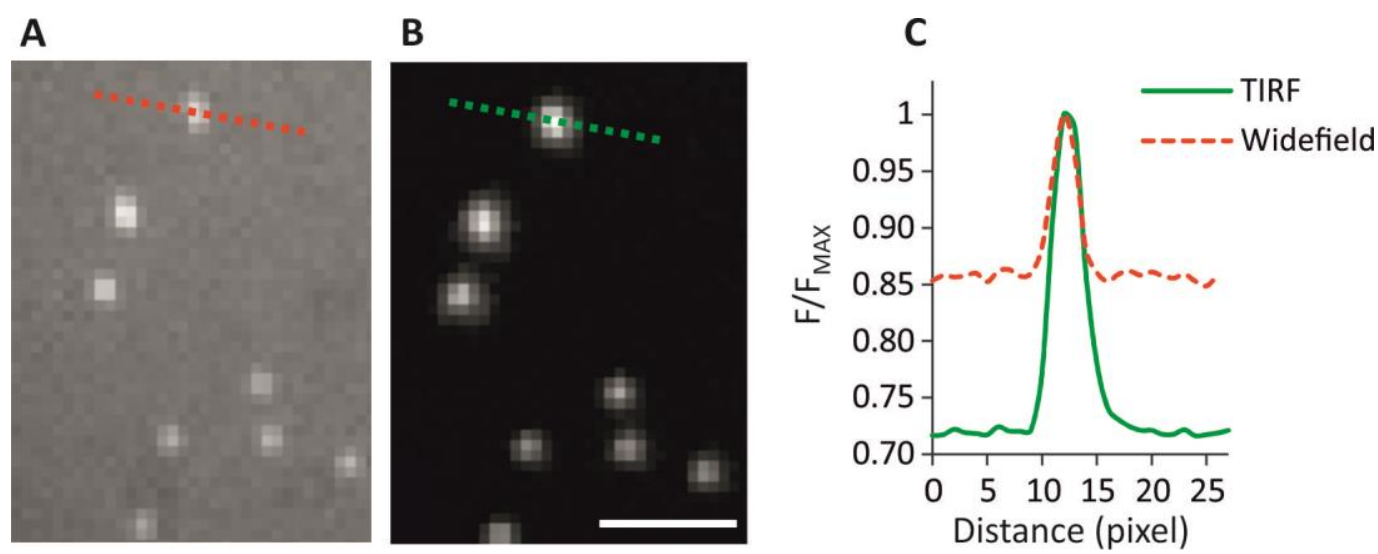

Figure 2-4 Widefield versus TIRF illumination.

Representative fluorescence images of immobilized yellow-green fluorescent beads, illuminated with the $488 \mathrm{~nm}$ line of the argon laser in widefield (A) and TIRF (B) mode, in the presence of a green fluorescent dye (Pyranine) in the bath solution. C) Normalized intensity cross-section through one single bead in image A (red line) and B (green line). As shown, the background signal was reduced in TIRF mode. Calculating SNR by dividing the background-subtracted peak intensity by the standard deviation of the fluorescence values in the background resulted in an SNR of 61.6 in widefield, and 142.5 in TIRF, indicating a 2.3 fold improvement of SNR in TIRF compared to widefield illumination.

\subsubsection{Coupling a UV Flash Lamp to TIRF Setup}

In order to provide a mercury lamp-based illumination source for the TIRF setup, a slider had been designed to direct the light from the lamp to the microscope through one of its side-openings. It consisted of an optical fiber holder, two mirrors (M1 and M2) and two lenses (80 and $75 \mathrm{~mm}$ focal length) (Figure 2-5). When the slider was coupled to the microscope, M2 blocked the laser path and only light from the mercury lamp could illuminate the sample. In order to equip the setup with an uncaging system, the mercury lamp was replaced with a Xenon-flash lamp. Moreover, to perform UV-uncaging simultaneously with illumination of the sample with lasers, M2 was replaced with a 425 nm longpass beamsplitter which allowed for more than $95 \%$ transmission of both laser lines while $99 \%$ of UV light was reflected. 


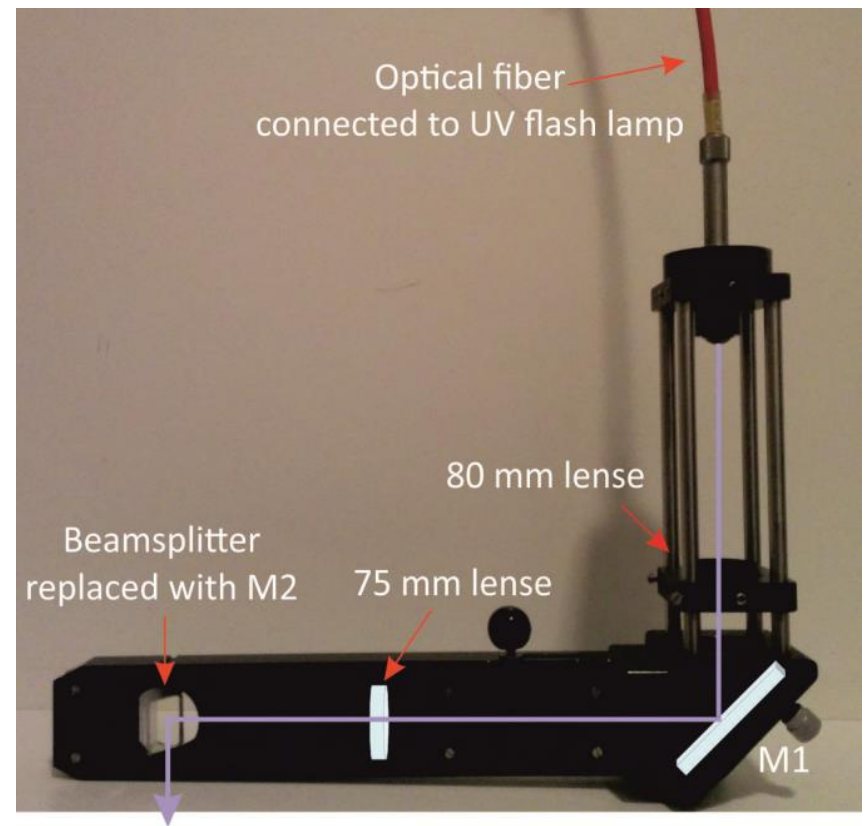

Figure 2-5 Coupling UV light to the setup through a side opening of the microscope.

The optical fiber connected to the flash lamp was coupled to a slider. The slider consisted of two lenses (focal lengths of 80 and $75 \mathrm{~mm}$ ) and two mirrors (M1 and M2). M2 was replaced with a beamsplitter (F48-425, AHF analysentechnik) for transmission of both lasers and reflection of UV light. By placing the slider in the microscope through one of its side openings, uncaging could be performed during imaging with lasers.

The flash lamp was controlled by Andor IQ2 software via TTL triggers. As a control experiment to test whether the UV light was aligned properly, $0.2 \mu \mathrm{m}$ yellow-green fluorescent beads were immobilized on PLL-coated coverslip and $40 \mu \mathrm{M}$ DM-Nitrophencalcium (provided by Dr. Kun-Han, Dep. Membrane biophysics, MPIbpc, Göttingen, Germany) as well as $2.5 \mu \mathrm{M}$ of the calcium indicator, Fluo-4, were added to the bath solution. The sample was illuminated in epifluorescence mode with the $488 \mathrm{~nm}$ line of the argon laser and a UV flash was triggered during imaging. An increase in Fluo-4 intensity was observed upon triggering the flash lamp, indicating that the UV light could efficiently uncage DM-Nitrophen-Ca ${ }^{2+}$ and release free calcium (Figure 2-6). 


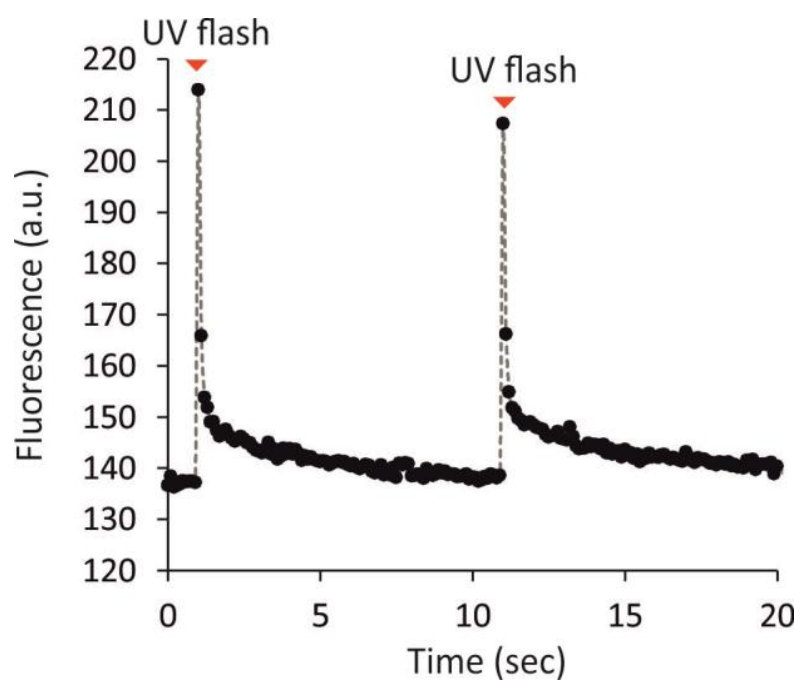

\section{Figure 2-6 Quality check of UV light alignments.}

Representative fluorescence response of the $\mathrm{Ca}^{2+}$ indicator, Fluo-4, in the solution to uncaging of DM Nitrophen-Ca ${ }^{2+}$ by two subsequent UV flashes. Release of $\mathrm{Ca}^{2+}$ by photolysis resulted in a transient increase of Fluo-4 fluorescence. Diffusion of $\mathrm{Ca}^{2+}$ out of the field of view diminished the fluorescence intensity.

\subsubsection{Solution Exchange System}

In order to perform fast solution exchange, the setup was equipped with a six channel perfusion valve control system (Table 2-2). The valve controller, which could be triggered manually or externally through Andor IQ2 software, provided synchronized opening/closing of multiple valves. To be able to exchange the whole bath solution in a short time, a custom-designed imaging chamber was constructed by the workshop of the Max-Planck Institute for Biophysical Chemistry (Göttingen, Germany). The chamber was designed to encompass a low bath volume $(<100 \mu \mathrm{l})$ and equipped with three inlets and one outlet. Moreover, a peristaltic pump was used for fast removal of solution. With this solution exchange system, more than $80 \%$ of the bath solution was exchanged in 200 msec.

\subsubsection{Probe Optimization}

In order to measure the two components of the electrochemical gradient in single synaptic vesicles, an appropriate $\mathrm{pH}$-sensitive and potentiometric probes are required. These probes should produce not only sufficiently fast signal in the response range of synaptic vesicles, but also require adequate photostability and should provide 
sufficiently high SNR. Finding a probe with the desired characteristics was the most challenging step in the development of the single-vesicle assay and required screening of many different probes (Table 2-7). Some of the tested probes are mentioned in the following sections. 
Table 2-7 List of $\mathrm{pH} /$ potentiometric probes tested in this study

\begin{tabular}{|c|c|c|c|c|}
\hline & Probe & $\begin{array}{c}E x c / E m \\
(n m)\end{array}$ & $p K_{a}$ & Pros and Cons \\
\hline \multirow{6}{*}{ 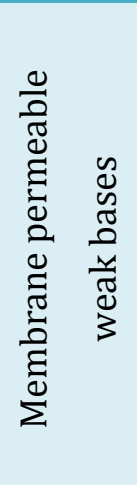 } & LysoSensor Green DND-189 & $443 / 505$ & $5.2^{1}$ & \multirow{4}{*}{$\begin{array}{ll}\checkmark & \text { Efficient labeling of acidic organelles in } \\
& \text { cells } \\
\times & \text { Cannot be removed from surrounding } \\
& \text { environment of SVs (high background) } \\
\times & \text { No response to ATP }\end{array}$} \\
\hline & LysoSensor Blue DND-167 & $373 / 425$ & $5.1^{1}$ & \\
\hline & LysoTracker Green DND-26 & $504 / 511$ & $\mathrm{ND}^{*}$ & \\
\hline & LysoTracker Red DND-99 & $577 / 590$ & ND & \\
\hline & Acridine Orange & $489 / 520$ & $10.5^{2}$ & $\checkmark$ Provide adequate signal in response to \\
\hline & ACMA & $419 / 483$ & $8.6^{3}$ & $\begin{array}{ll} & \text { acidification of SVs } \\
\times \quad \text { Poor SNR due to high background }\end{array}$ \\
\hline \multirow[b]{2}{*}{ 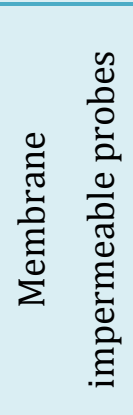 } & HPTS (Pyranine) & $403 / 511$ & $7.3^{1}$ & \multirow{2}{*}{$\begin{array}{ll}\checkmark & \text { Suitable } \mathrm{p} K_{\mathrm{a}} \text { for reporting acidification } \\
& \text { of SVs } \\
\times \quad & \text { Loading into the lumen of SVs requires } \\
& \text { high amount of the dye } \\
\times \quad \text { Loading into the lumen of SVs is a } \\
\text { multi-step process and has low yield for } \\
\text { single SV assay }\end{array}$} \\
\hline & SNARF 5F & $555 / 590$ & $7.2^{1}$ & \\
\hline \multirow{2}{*}{ 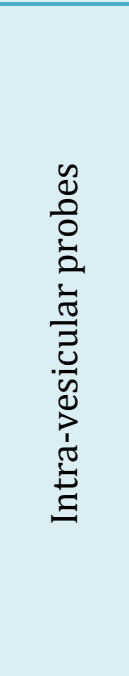 } & СypHer 5E & $650 / 665$ & $6.1^{3}$ & $\begin{array}{ll}\checkmark & \text { Provide adequate signal in response to } \\
& \text { acidification of SVs } \\
\times & \text { Low yield of labeling of the luminal } \\
& \text { domain of SV proteins } \\
\times & \text { Poor photostability }\end{array}$ \\
\hline & Super-ecliptic pHluorin & $490 / 515$ & $7.18^{4}$ & $\begin{array}{ll}\checkmark & \text { Suitable for quantitative measurement } \\
& \text { of pH gradient across the SV's } \\
& \text { membrane } \\
\checkmark & \text { High SNR } \\
\checkmark & \text { High photostability } \\
\times & \text { Low resolution at pH below } 6\end{array}$ \\
\hline \multirow{3}{*}{ 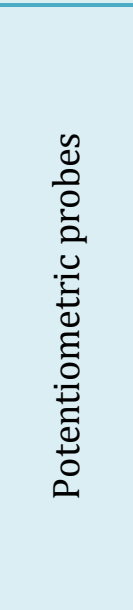 } & Di-8-ANEPPS & $498 / 713$ & ND & $\begin{array}{ll} & \text { Decent SNR } \\
\times & \text { Low voltage sensitivity }\end{array}$ \\
\hline & Oxonol VI & $599 / 634$ & ND & $\begin{array}{ll}\checkmark & \text { High voltage sensitivity } \\
\times & \text { Poor SNR due to high background }\end{array}$ \\
\hline & VF2.1.Cl & $522 / 535$ & ND & $\begin{array}{ll}\checkmark & \text { Suitable for quantitative measurement } \\
\text { of membrane potential across the SV's } \\
\text { membrane } \\
\checkmark & \text { Decent voltage sensitivity } \\
\checkmark & \text { High SNR } \\
\checkmark & \text { Decent photostability }\end{array}$ \\
\hline
\end{tabular}

${ }^{*}$ Not Determined 
${ }^{1}$ (Haugland, 2005)

${ }^{2}$ (Manente et al., 2008)

3 (Han and Burgess, 2010)

${ }^{4}$ (Sankaranarayanan et al., 2000)

\subsubsection{1 pH Sensitive Probes}

The equilibrium constant for protonation $\left(K_{\mathrm{a}}\right)$, or more commonly used, the negative logarithm of this constant $\left(\mathrm{p} K_{\mathrm{a}}\right)$, determines the $\mathrm{pH}$ range in which a given indicator can efficiently report pH changes. Eq. 2.1 shows the relationship between flourescence signal of a probe and its $\mathrm{p} K_{\mathrm{a}}$ (Sankaranarayanan et al., 2000):

$$
F=F_{0}+\frac{F_{\max }}{1+10^{\mathrm{p} K_{\mathrm{a}}-\mathrm{pH}}}
$$

where $F_{0}$ and $F_{\max }$ represent the offset and dynamic range, respectively. During synaptic activity, the lumen of early endocytosed SVs with $\mathrm{pH}$ of extracellular fluid $(\sim 7.4)$ abruptly switches to a more acidic $\mathrm{pH}(\sim 5.6)$ by the activity of V-ATPase (Miesenbock et al., 1998). Based on Eq. 2.1, pH probes with a $\mathrm{p} K_{\mathrm{a}}$ between 5 and 8 can report acidification of SVs from 7.4 to 5.6 by more than $20 \%$ change in their fluorescence signal (Figure 2-7). Therefore, different $\mathrm{pH}$-sensitive probes whose $\mathrm{p} K_{\mathrm{a}}$ fitted to this range were tested. These probes can be categorized into three groups:

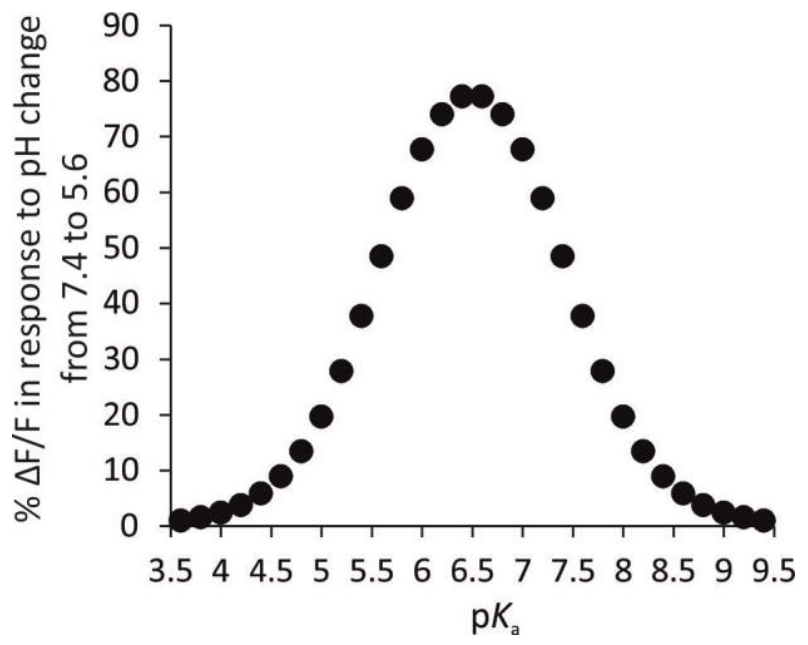

Figure 2-7 Effect of $\mathrm{p} K_{\mathrm{a}}$ of a $\mathrm{pH}$-sensitive probe on its fluorescence response to $\mathrm{pH}$ change from 7.4 to 5.6 .

The percentage of fluorescence change due to $\mathrm{pH}$ drop from 7.4 to 5.6 for probes with different $\mathrm{p} K_{\mathrm{a}}$ values was calculated using Eq. 2.1. As shown, a $\mathrm{pH}$ change in this range induces more than a $20 \%$ change in the fluorescence of probes with $\mathrm{p} K_{\mathrm{a}}$ values between 5 and 8 . 
LysoSensors and LysoTrackers: These probes are commonly used to stain acidic organelles in the cell (Han and Burgess, 2010). These membrane-permeable probes comprise amine groups, which are partially protonated at neutral $\mathrm{pH}$ and upon decrease in $\mathrm{pH}$ will be fully protonated. This in turn helps in accumulation and retention of the probes inside acidic organelles, and therefore results in higher signal intensity of these compartments compared to their surrounding environment. Moreover, LysoSensor protonation dequenches the dye molecule by blocking electron transfer from amine groups and results in higher fluorescence intensity (Haugland, 2005). This feature makes LysoSensors more beneficial than LysoTrackers whose fluorescence is largely pH-independent.

In order to test whether these probes can indeed label acidic organelles in a cell, PC12 cells were stained with different LysoTrackers and LysoSensors. For this purpose, cells plated on PLL-coated glass coverslips were incubated with DMEM (Dulbecco's Modified Eagle Medium, Life technologies) containing different concentrations of the dyes for 45 min at $37^{\circ} \mathrm{C}$. Then the cells were washed with dye-free medium and imaged using a confocal microscope (Table 2-6). All the tested LysoSensors and LysoTrackers could efficiently label the intracellular acidic organelles. According to manufacturer, high concentrations of LysoTrackers can alkalinize the lumen of organelles. Therefore, the lowest concentration which worked best for labeling PC12 cells was used to test whether these probes can report acidification of purified SVs. For bulk acidification assays, $100 \mu \mathrm{g}$ of crude SVs were suspended in $1 \mathrm{ml}$ of dye-containing K-gluconate buffer in $10 \mathrm{~mm}$ pathway quartz cuvettes and acidification assays were performed using a Fluorolog III spectrofluorometer (Table 2-6). The fluorescence of the sample was continuously monitored, during which $1.2 \mathrm{mM}$ ATP and $120 \mathrm{mM} \mathrm{KCl}$ were consecutively added to the sample. From the tested probes of this category, results of LysoSensor Green DND-189 and LysoTracker Red DND-99 are shown in Figure 2-8. Had the probes been suitable for acidification measurement, an increase in fluorescence intensity should have been observable upon ATP addition. However, acidification of purified SVs with ATP did not induce any detectable signal change of the sample. Addition of chloride, which is known to induces greater acidification (Stobrawa et al., 2001), also did not increase the fluorescence intensity of the sample. This implies that the fluorescence increase upon accumulation of the dye in acidified lumen of SVs was not large enough to 
overcome the high signal coming from free dye in the solution. Therefore, these probes were not pursued for single-vesicle assays.
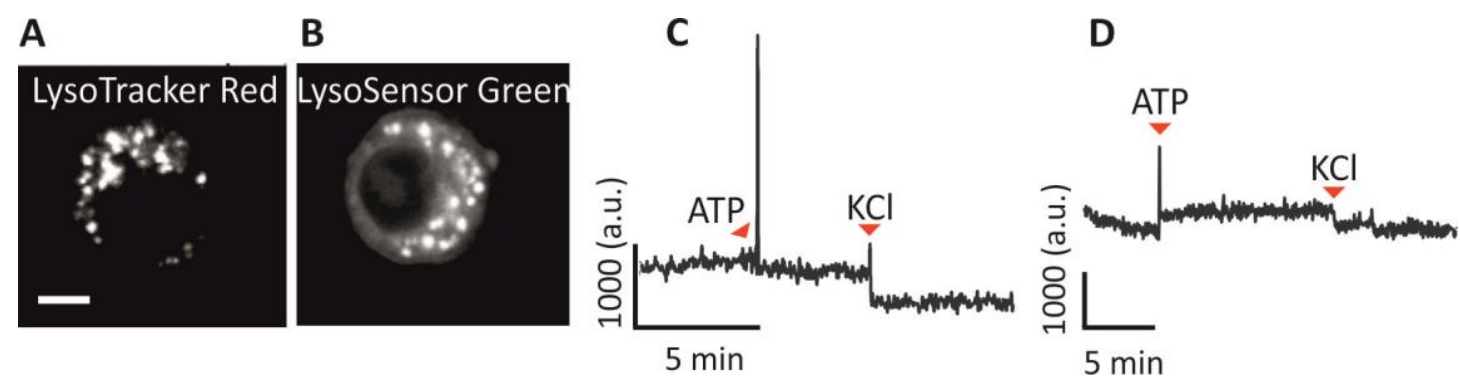

Figure 2-8 Response of LysoTracker Red DND-99 and LysoSensor Green DND-189 to SV acidification.

Representative fluorescence images of PC12 cells labeled with $50 \mathrm{nM}$ of LysoTracker Red DND99 (A) and $4 \mu \mathrm{M}$ of LysoSensor Green DND-189 (B). Scale bar is $50 \mu \mathrm{m}$. Acidification bulk assay was performed with $100 \mu \mathrm{g}$ of crude SVs and $50 \mathrm{nM}$ LysoTracker Red DND-99 (C) or $100 \mathrm{nM}$ of LysoSensor Green DND-189 (D) in glycine buffer. Excitation and emission wavelengths for each dye were set according to Table 2-7. No fluorescence change was observed upon addition of 1.2 mM ATP or $120 \mathrm{mM} \mathrm{KCl}$ to the sample.

Acridine dyes: These dyes are also permeant weak bases, but the mechanism by which they report $\mathrm{pH}$ changes is completely different from the previously mentioned probes. Despite their extensive use in biological systems, these probes cannot report absolute $\mathrm{pH}$ in the physiological range due to their high $\mathrm{p} K_{\mathrm{a}}$. As an example of tested acridine dyes, the mechanism and results obtained with acridine orange (AO) is discussed in this section.

AO when diluted in aqueous solutions is mainly monomeric, but upon increase in concentration will form dimers, trimers or higher order oligomers. The equilibrium between monomeric and dimeric/oligomeric $\mathrm{AO}$ is also affected by $\mathrm{pH}$ of its surrounding environment. While at neutral $\mathrm{pH}$ monomeric $\mathrm{AO}(\mathrm{mAO})$ is the dominating form, a decrease in $\mathrm{pH}$ shifts the equilibrium towards dimers/oligomers (Palmgren, 1991). Hence, when $\mathrm{AO}$ is in the lumen of an organelle which acidifies, aggregated AO will be formed in the lumen, which unlike monomers cannot permeate the bilayer membranes (Figure 2-9A). This leads to an increase in the population of aggregated AO, and in turn a decrease in the number of monomers (Zoccarato et al., 1999). Since these forms of A0 have distinct excitation and emission maxima, the acidification process can be monitored by measuring the optical response of either of these forms. In conventional 
bulk acidification assays, emission (Bellocchio, 2000) or absorbance (Hell et al., 1990; Preobraschenski et al., 2014) of mAO is monitored.

Acidification bulk assays were performed with $10 \mu \mathrm{M}$ AO as described above for LysoSensors and LysoTrackers. The excitation and emission wavelengths were set according to the spectral characteristics of mAO at 491 and $530 \mathrm{~nm}$, respectively. As expected, addition of ATP led to a decrease in emission intensity of mAO (Figure 2-9B). This is due to a decrease in the population of mAOs upon their conversion to aggregates in acidified lumen of SVs. Addition of ammonium sulfate $\left(\left(\mathrm{NH}_{4}\right)_{2} \mathrm{SO}_{4}\right)$ in turn, recovered the fluorescence signal which is due to capturing of protons in the lumen and subsequently alkalinization of the $\mathrm{SV}$ lumen by $\mathrm{NH}_{3}$. Next, $\mathrm{AO}$ was used for a microscopy-based acidification assay. For this purpose, immobilized SVs on PLL-coated coverslips were imaged in the presence of AO in the bath solution using the TIRF setup. SVs were excited by the $488 \mathrm{~nm}$ line of the argon laser and their emission was collected through a 515/30 nm emission filter. Since mAO is highly fluorescent in neutral $\mathrm{pH}$, a low concentration of $\mathrm{AO}(1 \mu \mathrm{M})$ was used for imaging. However, the SNR in AO images, although significantly improved in TIRF mode, was still very poor due to high background signal. This was probably due to binding of $\mathrm{mAO}$ to the hydrophilic surface of PLL-coated coverslips. Another hindrance to single-vesicle application of AO was that addition of even small volumes of solution further diminished the signal of single SVs by diluting the dye in the field of view. Consequently, AO did not meet the requirements of an appropriate probe for single vesicle imaging. It should be noted that the drawbacks to $\mathrm{AO}$ are probably a feature of other membrane-permeable probes as well. As long as free dye molecules cannot be removed from the bath solution, a high background signals from the bath solution decreases the SNR. Even in cases of probes like LysoSensor Green DND-189, whose $\mathrm{p} K_{\mathrm{a}}$ is low and the dye is only weakly fluorescent in neutral $\mathrm{pH}$, the SNR remains low because of the extremely small size of SVs, which cannot accommodate more than a small portion of dye molecules in its lumen or surface. Therefore, membrane-impermeable probes were tested to eliminate the background. 
A

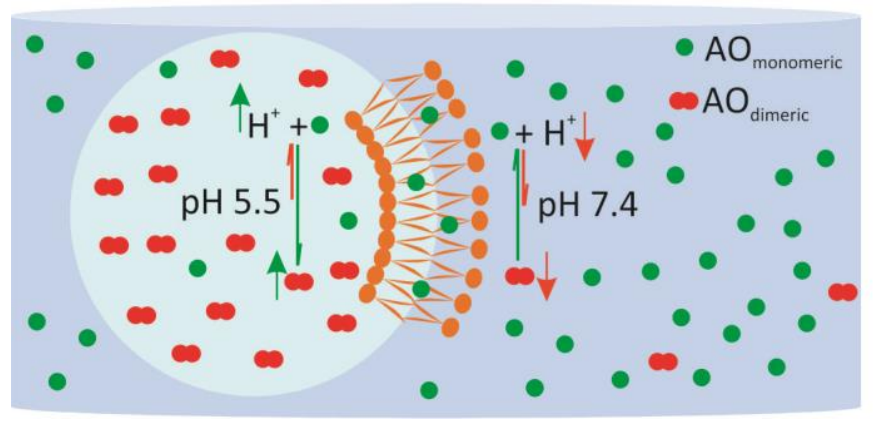

B

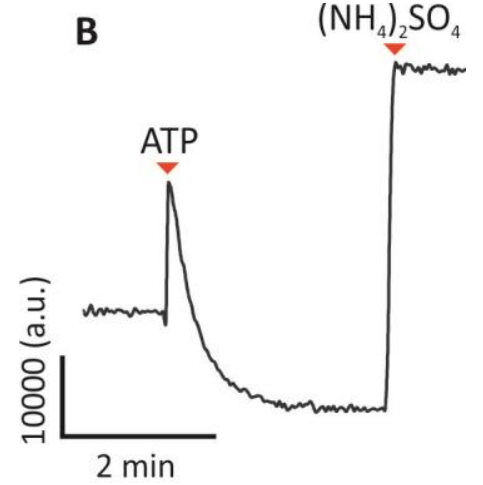

Figure 2-9 Acridine Orange mechanism and its response to acidification.

A) Schematic representation of the proposed mechanism by which AO reports acidification of the lumen of SVs. As depicted, $\mathrm{AO}_{\text {monomeric }}$ (green circles) is membrane-permeable and highly abundant at alkaline $\mathrm{pH}$ (7.4). On the contrary, $\mathrm{AO}_{\text {dimeric }}$ (red double-circles), which is the dominant form of AO at low luminal pH (5.5), cannot pass the lipid bilayer of the SVs and is trapped in the acidified lumen. Upon acidification of SVs, the number of $\mathrm{AO}_{\text {monomeric }}$ in the solution decrease while the number of $\mathrm{AO}_{\text {dimeric }}$ in the vesicular lumen will increase. B) Acidification bulk assay with $100 \mu \mathrm{g}$ of crude SVs and $10 \mu \mathrm{M}$ AO in glycine buffer containing 120 $\mathrm{mM} \mathrm{KCl}$. Addition of $2.4 \mathrm{mM} \mathrm{Mg-ATP}$ dramatically decreased the fluorescence of the sample, indicating that the lumens of SVs were acidified. Subsequent addition of $50 \mathrm{mM}\left(\mathrm{NH}_{4}\right)_{2} \mathrm{SO}_{4}$ recovered the fluorescence signal by alkalinizing the vesicular lumen.

ii. Membrane-Impermeable Probes

Pyranine, also known as HPTS (8-Hydroxypyrene-1,3,6-Trisulfonic Acid) is highly hydrophilic and has a $\mathrm{p} K_{\mathrm{a}}$ of $\sim 7.3$. Despite its membrane-impermeability, it is widely used for intracellular $\mathrm{pH}$ measurements due to its high $\mathrm{pH}$ resolution in the physiological range, fast response, low leakage across intracellular membranes and low toxicity (Overly et al., 1995). SVs were loaded with Pyranine via fusion with liposomes encapsulating high concentrations of the dye. For liposome preparation, a lipid mixture was prepared, as described by (Milovanovic et al., 2015), by mixing DOPC (1,2-dioleoylsn-glycero-3- phosphocholine), DOPS (1,2-dioleoyl-sn-glycero-3-phospho-L-serine) and cholesterol (synthetic lipids from Avanti Polar Lipids) all in chloroform in a 65:10:25 molar ratio. The chloroform was evaporated and the resulting lipid film was then resuspended to $8 \mathrm{mM}$ total lipid concentration in buffer containing HEPES (5 mM, pH 7.4), glycine (300 mM), and Pyranine ( $5 \mathrm{mM}$ ) using solid-glass beads (Sigma-Aldrich, MO, USA), and extruded through polycarbonate filters with a $100 \mathrm{~nm}$ pore diameter (Avanti Polar Lipids). As shown in Figure 2-10A, Pyranine was efficiently encapsulated inside liposomes, as the fluorescence of liposome suspension could only be quenched 
with $p$-Xylene-bis-pyridinium bromide (DPX), a Pyranine fluorescence quencher, in the presence of the detergent Triton X-100. Next, the stabilized SNARE acceptor complex (provided by Dr. Julia Preobraschenski, Dep. of Neurobiology, MPIbpc, Göttingen, Germany), was reconstituted into Pyranine loaded liposomes as described by (Preobraschenski et al., 2014). Briefly, proteins were mixed with liposomes in the presence of $10 \%(\mathrm{w} / \mathrm{v})$ n-Octyl- $\beta$-DGlucopyranoside and $5 \mathrm{mM}$ Pyranine at a protein:lipid molar ratio of $\sim 1: 500$, followed by overnight dialysis at $4^{\circ} \mathrm{C}(2 \mathrm{kDa} \mathrm{MWCO}$, SpectraPor) in the same buffer used for liposome preparation (including $5 \mathrm{mM}$ Pyranine), supplemented with 2 g of BioBeads (BioRad). Thereafter, proteoliposomes were fused with SVs for $45 \mathrm{~min}$ at room temperature as described by (Holt et al., 2008) while still $5 \mathrm{mM}$ Pyranine was included in the fusion mixture. Excess dye molecules were removed by running the sample on prepacked size exclusion columns (PD10, GE). For the acidification bulk assay, $300 \mu \mathrm{l}$ of fused SVs eluted from PD10 columns were mixed with $120 \mathrm{mM} \mathrm{KCl}$ buffer containing 5 mM HEPES (pH 7.4), and emission of the sample was collected at $509 \mathrm{~nm}$ while it was excited at $460 \mathrm{~nm}$. As shown in Figure 2-10B, addition of ATP did not produce any detectable signal. The encapsulation of Pyranine inside SVs/liposomes was verified by subsequent addition of DPX and Triton X-100 (TTX). These results imply that the efficiency of SV fusion for this purpose is most likely not sufficient, and the sample still contained a large fraction of dye-containing liposomes which were not fused with SVs. It was concluded that the loading of SVs was a multi-step process, required a large amount of the dye, and subtle inefficiencies in each step could have eventually resulted in ineffective loading.
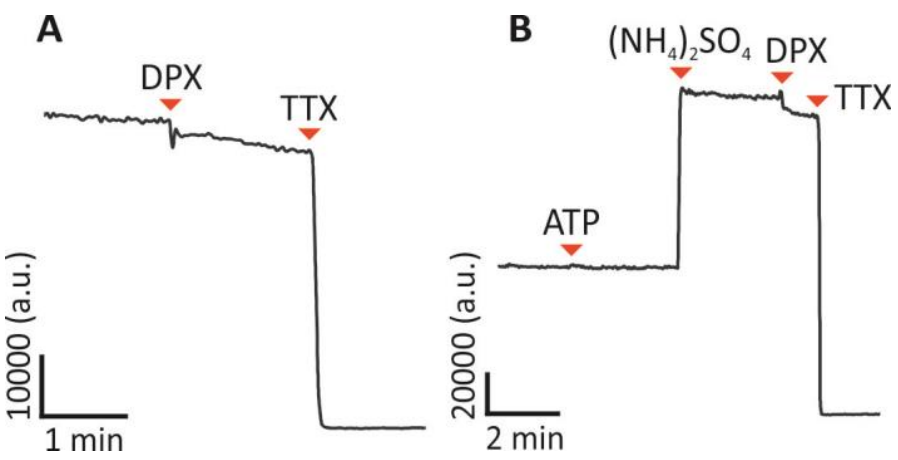

Figure 2-10 Loading of Pyranine into SVs via fusion with liposomes containing dye.

A) Fluorescence trace of liposomes encapsulating Pyranine. $20 \mathrm{mM}$ DPX, the Pyranine quencher, could only quench fluorescence of Pyranine in the presence of Triton X-100 (TTX) $(1 \%(\mathrm{v} / \mathrm{v})$ final concentration), indicating that dye was encapsulated in the liposomes. B) Addition of 2.4 
mM ATP did not produce detectable signal change in the sample. Subsequent addition of $20 \mathrm{mM}$ DPX and 1\% (v/v) TTX showed that the dye was efficiently loaded into liposomes/fused SVs.

\section{iii. Intravesicular Probes}

Synaptic vesicles possess a high density of integral membrane proteins (Takamori et al., 2006). In addition to loading of vesicles with a probe, one way to target a probe to the lumen of SVs is to tag it to the luminal domain of one of these proteins. For this purpose, two approaches were tested: labeling the luminal domain of synaptotagmin 1 with a CypHer5E-coupled antibody raised against $\mathrm{N}$-terminus of protein, and purification of synaptic vesicles from transgenic mice expressing a fluorescent protein in the lumen of SVs.

CypHer5E: $\mathrm{pH}$-sensitive cyanine dyes have $\mathrm{p} K_{\mathrm{a}}$ of around 6.1, are non-fluorescent at $\mathrm{pH} 7.4$ and fluoresce upon protonation at lower $\mathrm{pH}$. In order to target CypHer5E to the lumen of SVs, the luminal domain of synaptotagmin 1 was labeled with a CypHer5E labeled antibody. For this purpose, synaptosomes were isolated from rat cerebral cortex by the previously described protocol (Fischer von Mollard et al., 1991). Labeling of SVs was then performed using the protocol described by (Rizzoli et al., 2006) with small modifications. Briefly, purified synaptosomes were diluted in sodium buffer (Table 2-1), and after incubation at $37^{\circ} \mathrm{C}$ for $5 \mathrm{~min}, 10 \mu \mathrm{g}$ of labeled antibody as well as $1.3 \mathrm{mM}$ of $\mathrm{CaCl}_{2}$ were added. After $2 \mathrm{~min}$, synaptosomes were stimulated by $50 \mathrm{mM} \mathrm{KCl}$ for $5-15$ min and then cooled on ice. They were then centrifuged at $8700 \times g$ for $10 \mathrm{~min}$ and washed once with sodium buffer. This was done by resuspending the synaptosomes and repeating the centrifugation step. A small fraction of synaptosomes were immobilized on a glass coverslip to check for labeling efficiency (Figure 2-11A). In order to obtain labeled SVs, the rest of the synaptosomes were disrupted by diluting them in a nine-fold greater volume of MilliQ-purified water, followed by three strokes of homogenization at $2000 \mathrm{rpm}$ in a Teflon-glass homogenizer. The suspension was then centrifuged at 20000 $\mathrm{x} g$ for $25 \mathrm{~min}$. The supernatants containing labeled SVs were used for the acidification bulk assay. As shown in Figure 2-11, following this protocol, synaptosomes were successfully labeled with CypHer5E. However in acidification bulk assays with labeled SVs released from these synaptosomes, only a very small increase of CypHer5E signal was observed upon addition of ATP, which was reversed by addition of $\left(\mathrm{NH}_{4}\right)_{2} \mathrm{SO}_{4}$. This indicates that the final yield of the CypHer5E -labeling of SVs was very low, possibly due 
to loss of SVs during the osmotic lysis. In the next step, the labeled SVs were immobilized on PLL-coated coverslips. The number of total fluorescent SVs in each field of view was very low. In addition, the photostability of the labeled-SVs was poor. Therefore, although SVs could be labeled with CypHer5E and this probe was capable of reporting acidification of SVs, this approach was not further pursued for the singlevesicle assay.

A

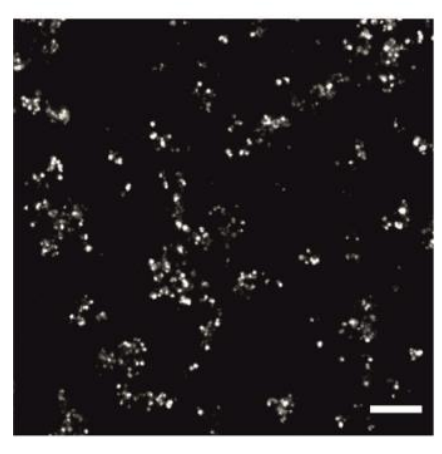

\section{B}

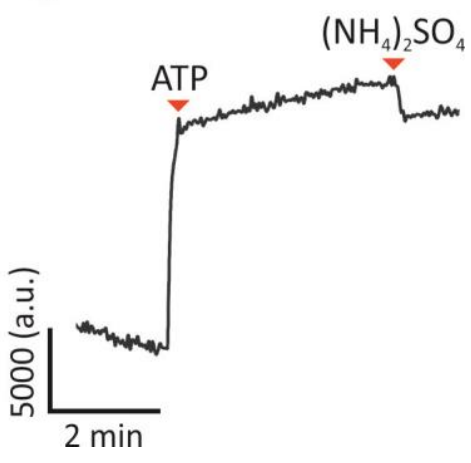

\section{Figure 2-11 CypHer5E -labeling of SVs.}

A) Representative image of synaptosomes after labeling with CypHer5E-labeled antibody against luminal domain of synaptotagmin 1 (105 103CpH, Table 2-5). Scale bar is $10 \mu \mathrm{m}$. B) Representative fluorescence trace of CypHer5E-labeled SVs in glycine buffer containing $120 \mathrm{mM}$ $\mathrm{KCl}$ after osmotic lysis of synaptosomes. Addition of $2.4 \mathrm{mM}$ ATP led to a small increase in fluorescence, indicating acidification of SV's lumen. The fluorescence change was reversed by 40 $\mathrm{mM}\left(\mathrm{NH}_{4}\right)_{2} \mathrm{SO}_{4}$ which alkalinized the lumen.

pHluorin: In 1998, two pH-sensitive variants of green fluorescent protein (GFP), referred to as pHluorin, were developed by specific substitutions in the proton-relay network of the GFP protein: ratiometric pHluorin and ecliptic pHluorin (Miesenbock et al., 1998). These mutants of GFP have been widely used to study synaptic transmission due to the advantage that they can be targeted to specific organelles or to the plasma membrane (Miesenbock, 2012). Similar to wild type GFP, these proteins have a bimodal excitation spectrum with peaks at 395 and $475 \mathrm{~nm}$. While ratiometric pHluorin shows an excitation change between pH 7.5 and 5.5, ecliptic pHluorin loses fluorescence at both excitation wavelengths as $\mathrm{pH}$ is lowered. In this thesis, a mutated variant of ecliptic pHluorin, namely super-ecliptic pHluorin ( $\mathrm{spH}$ ), was tested, for which spH-21 transgenic mice were kindly provided by Prof. V.N. Murthy (Department of Molecular and Cellular Biology, Harvard University, USA) and Dr. W. Tyler (Virginia Tech Carilion Research Institute, USA). In these mice, $\mathrm{spH}$ is tagged to the luminal domain of VAMP2, and thus is 
sensitive to the luminal $\mathrm{pH}$. Both ecliptic pHluorin and $\mathrm{spH}$ exist in two conformers: the form which is best excited at $\sim 475 \mathrm{~nm}$ and loses its fluorescence completely (eclipsed) at $\mathrm{pH}$ below 6 , and a less $\mathrm{pH}$-sensitive form whose excitation is slightly blue-shifted $(\sim$ $395 \mathrm{~nm}$ ) but retains its fluorescence at pH below 6. The difference between ecliptic pHluorin and $\mathrm{spH}$ is that two additional substitutions in the chromophore of $\mathrm{spH}$ effectively populated the more pH-sensitive (ecliptic) species and red-shifted its excitation peaks to 405 and $491 \mathrm{~nm}$. This in turn resulted in a 5.9-fold increase in the fluorescence intensity of spH compared to ecliptic pHluorin (Miesenbock, 2012).

To verify the spectral profile of $\mathrm{spH}$, the excitation and emission of $1 \mu \mathrm{g}$ of purified protein (provided by Dr. Andrew Woehler, Dep. Membrane biophysics, MPIbpc, Göttingen, Germany) was collected while the pH was titrated in $300 \mathrm{mM}$ glycine buffer containing $10 \mathrm{mM}$ MOPS. As expected, two excitation peaks were observed at 405 and $491 \mathrm{~nm}$, with the latter showing pH-sensitive fluorescence (Figure 2-12). To determine the $\mathrm{p} K_{\mathrm{a}}$ of the protein, the averaged fluorescence intensity of the sample from two measurements was normalized to the maximum fluorescence (obtained at $\mathrm{pH} 9.5$ ) and plotted against $\mathrm{pH}$. Data were fit to Eq. 2.1. This resulted in a $\mathrm{p} K_{\mathrm{a}}$ of 7.2, which is close to the previously reported value for spH (7.18) (Sankaranarayanan et al., 2000).

Next, SVs were purified from spH-21 mice (spH-SV) using the protocol described in section 2.6.1. To ensure that the spectral characteristics of VAMP2-tagged spH (also known as synaptopHluorin) resemble that of the soluble protein, a $\mathrm{pH}$ titration was performed with purified spH-SVs as well. For this purpose, immobilized spH-SVs were imaged at different $\mathrm{pH}$ values (5.5-7.4) using the TIRF setup. For each $\mathrm{pH}$ measurement, the vesicles were equilibrated for $2 \mathrm{~min}$ in the bath solution (10 mM MOPS, $10 \mathrm{mM}$ MES, $300 \mathrm{mM}$ glycine), after which they were excited by the $488 \mathrm{~nm}$ line of the argon laser and their fluorescence was collected through a 515/30 nm emission filter. In addition, to facilitate full proton equilibrium between the lumen of SVs and the bath solution, $10 \mu \mathrm{M}$ carbonyl cyanide-4-(trifluoromethoxy) phenylhydrazone (FCCP), a proton ionophore (Table 2-4), was applied to the bath solution. The averaged fluorescence intensity of vesicles at all pH points were normalized to the fluorescence at $\mathrm{pH} 7.4$, plotted as a function of $\mathrm{pH}$ and fit to Eq. 2.1. The same $\mathrm{p} K_{\mathrm{a}}$ (7.23) was obtained in single spH-SVs confirming that the $\mathrm{pH}$ sensitivity of $\mathrm{spH}$ in the lumen of SVs was intact (Figure 2-12D). 

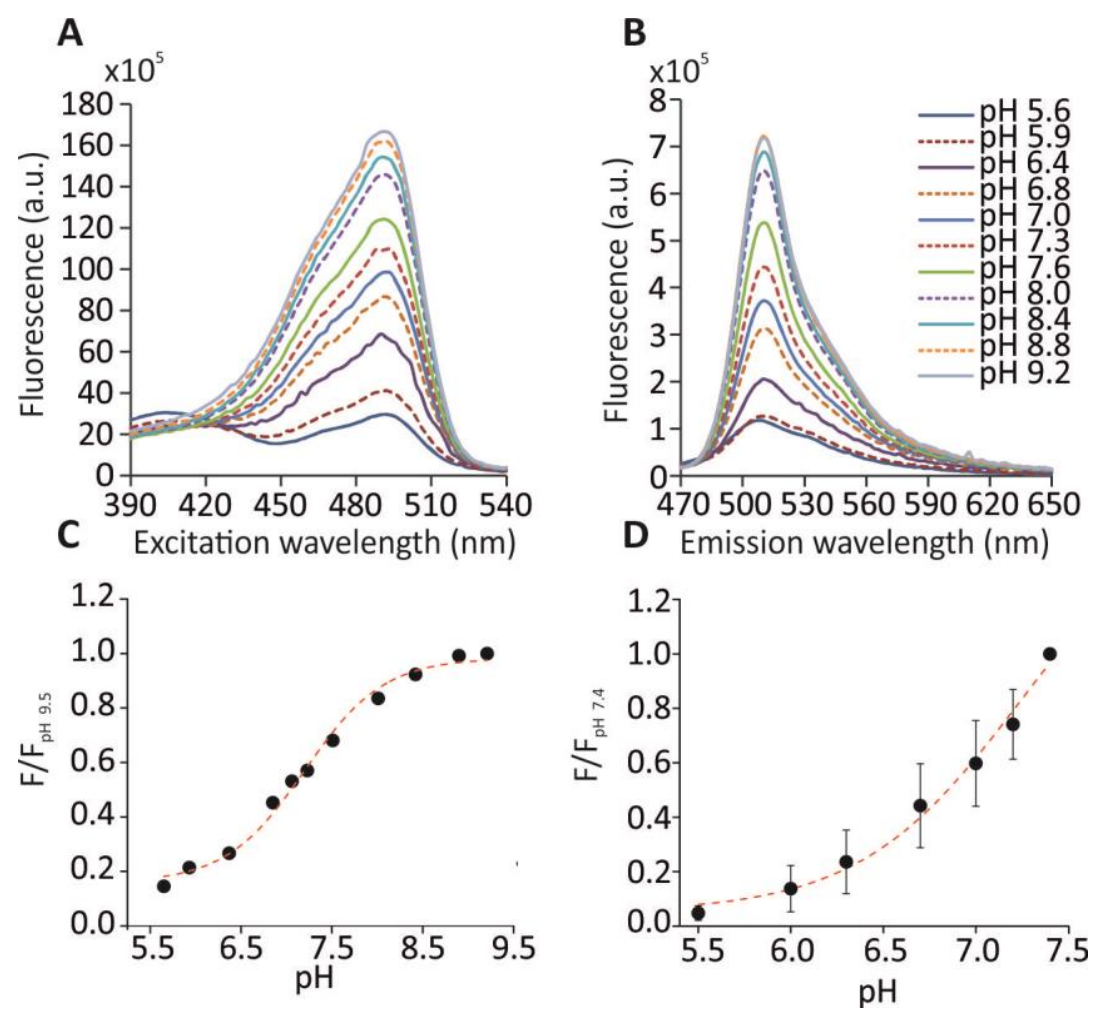

Figure 2-12 Spectral characteristics of spH.

Excitation (A) and emission (B) spectra of purified $\mathrm{spH}$ in glycine buffer. For the excitation spectra, emission was collected at $560 \mathrm{~nm}$, and for the emission spectra the sample was excited at $450 \mathrm{~nm}$. Fluorescence-pH calibration curve of purified protein in solution (C), and in spH-SVs (D). Red lines represent fitting to the data according to Eq. 2.1 which resulted in $\mathrm{p} K_{\mathrm{a}}$ of $7.20 \pm$ 0.05 and $7.23 \pm 0.15$ (SD) in C and D, respectively. Error bars in D represent SD ( $n=518$ SVs, compiled from 3 independent experiments).

Moreover, to count the number of $\mathrm{spH}$ proteins in single spH-SVs, a photobleaching experiment was performed with purified vesicles (in collaboration with Dr. Andrew Woehler, Dep. Membrane Biophysics, MPIbpc, Göttingen, Germany). For this purpose, immobilized spH-SVs were continuously excited in TIRF mode with the $488 \mathrm{~nm}$ laser. The laser output power was set to $\sim 20 \mathrm{~mW}$ and imaging was performed at $5 \mathrm{~Hz}$ for 100 s. As shown in Figure 2-13A, discrete bleaching steps were observed in fluorescence traces of single SVs. In order to determine the bleaching step size, the photobleaching image was first divided into 4 regions and the average size of a single bleaching step was measured from each quadrant. No significant difference was observed in step size from different regions of the image (ANOVA one-way analysis at probability level of 0.05), indicating uniform TIRF illumination. All the values from the entire image were pooled and a mean fluorescence intensity of 5,262 (a.u.) was obtained for one single bleaching step. Next, the total number of bleaching steps for single SVs was determined by dividing 
their initial intensity by the size of one bleaching step. The values from all spH-SVs were pooled into a histogram. Although a small portion of fluorescent particles ( 20\%) showed only 1 or 2 bleaching steps, the majority of vesicles showed more bleaching steps. Poisson fitting to the second distribution in the histogram indicated that on average, a single spH-SV contained 4.5 molecules which corroborates with the previously reported copy-number of spH (4.4) in purified spH-SVs (Gadd et al., 2012).

The value obtained by the photobleaching experiment was verified by mass spectrometry (in collaboration with Mahdokht Kohansal Nodehi, Dep. Neurobiology, MPIbpc, Göttingen). Briefly, the purified spH-vesicles from three SV preparations were analyzed via SDS-PAGE (Schagger, 2006) (ppendix 2). The spH-tagged VAMP2 was clearly separated from the non-tagged proteins due to difference in molecular weight (Figure 2-13D). spH-tagged VAMP2 proteins were then cut out of the gel and each was run in three technical replicates through mass spectrometer. By intensity-based absolute quantification (iBAQ) the average number of $\mathrm{spH}$ molecules in a single SV was estimated to be $4.1 \pm 0.7$ (SD), which is close to the results obtained from photobleaching measurements.
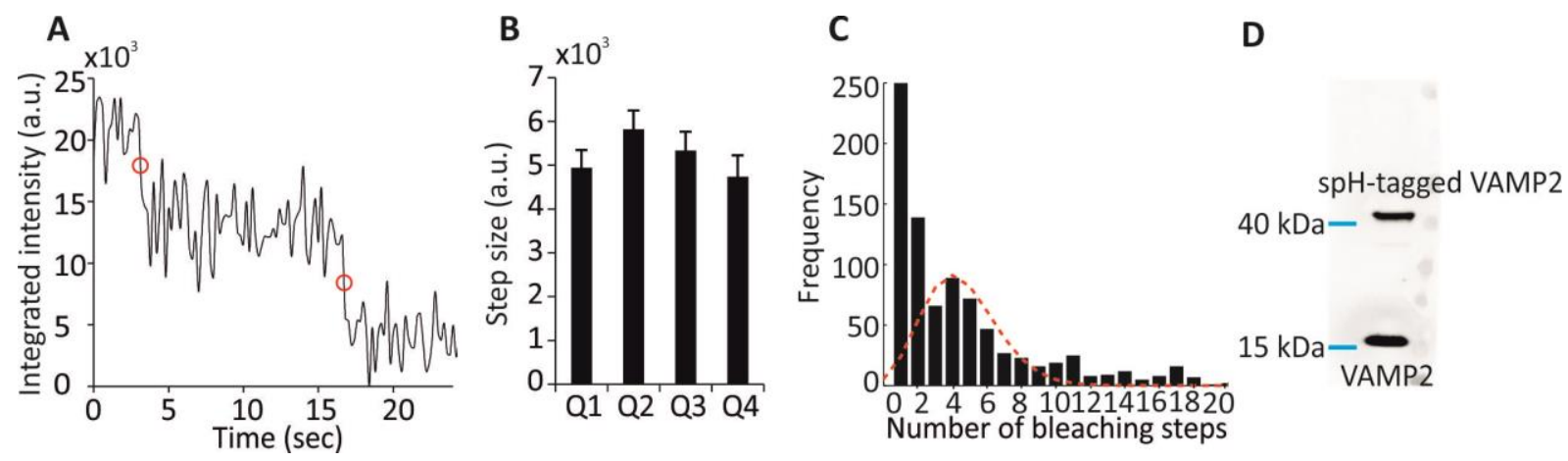

\section{Figure 2-13 Quantification of the number of fluorophores in single SVs.}

A) Representative trace of photobleaching of a single $\mathrm{SpH}$-vesicle. Photobleaching steps are indicated by red circles. B) In order to determine the step size, the photobleaching image was first divided into 4 regions and the average size of one discrete bleaching step was measured from each quadrant (Q). Error bars represent SEM of 13-20 photobleaching steps. C) The values from all the SpH-SVs were pooled into a histogram. The red line shows poisson fitting to the second distribution in this histogram. D) Immunoblot analysis (Appendix 2) of purified spH-SVs using an Oyster650-labeled antibody against VMAP2 (104 211C5, Table 2-5). The membranes were scanned with $488 \mathrm{~nm}$ laser line of the FLA-7000 scanner (Fujifilm).

In order to check the fluorescence response of $\mathrm{spH}$ to SV acidification, a bulk assay was performed with $100 \mu \mathrm{g}$ of crude spH-SVs in $1 \mathrm{ml}$ K-gluconate buffer containing 120 
$\mathrm{mM}$ chloride. The sample was excited at $475 \mathrm{~nm}$ and emission was collected at $530 \mathrm{~nm}$. As shown in Figure 2-14A, a decrease in fluorescence was observed after ATP addition which was reversed by Bafilomycin A1, a specific blocker of the V-ATPase, indicating that the fluorescence change was ATP-specific. Moreover, by immobilizing spH-SVs on PLL-coated coverslips, single fluorescent vesicles were observed with much higher SNR compared to the dye-based assays described above (Figure 2-14B). In addition, to check for photostability of the probe, SVs were imaged continuously for $20 \mathrm{~s}$ with a frame rate and excitation intensity reflecting the settings utilized for acidification measurements. Less than 15\% photobleaching was observed within the experimental timeframe (Figure 2-14C). These data demonstrate that the $\mathrm{pH}$ sensitivity and photostability of spH was sufficient to measure acidification of single SVs.
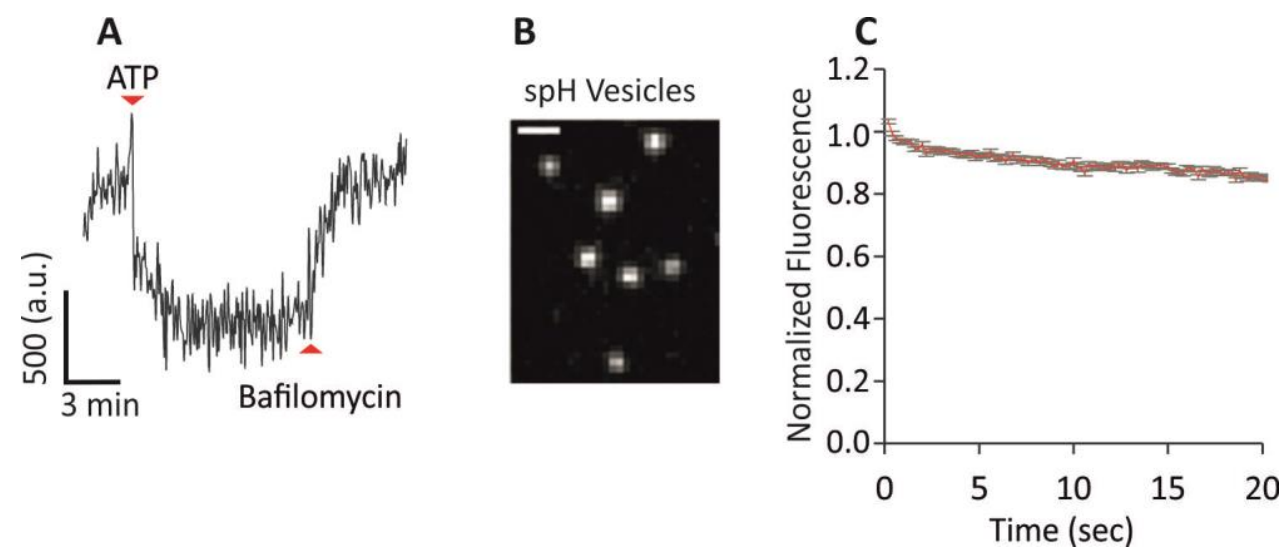

Figure 2-14 $\mathrm{spH}$ as a $\mathrm{pH}$ probe for single vesicle acidification assay.

A) Acidification bulk assay with crude SVs. spH-SVs were first acidified with $1.2 \mathrm{mM}$ ATP, which resulted in quenching of $\mathrm{spH}$, and then the V-ATPase was blocked by $100 \mathrm{nM}$ of Bafilomycin A1, a specific blocker of the V-ATPase, which reversed the fluorescence change. B) Representative image of immobilized spH-SVs on PLL-coated coverslips. Vesicles were excited with the $488 \mathrm{~nm}$ line of the argon laser in TIRF mode, and their emission was collected through a 515/30 nm filter. Scale bar is $1 \mu \mathrm{m}$. C) Trace of averaged integrated fluorescence of spH-SVs showing the extent of photobleaching over the experimental timeframe. The same laser and image acquisition setting as in the acidification experiments were used for the photobleaching measurements. Error bars represent SEM of $n$ single SVs, $n=2,053$.

\subsubsection{Potentiometric Probes}

In order to measure electrical gradient across the membrane of SVs, three probes with different response mechanisms were tested; Di-8-ANEPPS, a member of the socalled fast-response probes, Oxonal VI, a member of slow-response probes, and VF2.1.Cl 
which belongs to the family of molecular wire photo-induced electron transfer (PeT)based voltage sensors.

Fast-response probes respond to changes in their surrounding electrical field by changes in their electronic structure which subsequently alter their fluorescence properties. Despite their high temporal resolution (millisecond), the magnitude of their potential-dependent fluorescence change is relatively small (Haugland, 2005). The tested dye of this family, Di-8-ANEPPS (1-(3-sulfonatopropyl)-4-[ $\beta[2-($ di-n-octylamino)6-naphthyl]vinyl]pyridinium betaine), exhibits a $10 \%$ per $100 \mathrm{mV}$ changes in its fluorescence intensity (Zhang et al., 1998). To test this probe, purified SVs were labeled with different concentrations of the dye by including the dye in the bath solution during immobilization of the SVs on the coverslips. Since the dye was retained in the outer leaflet of the membrane, the excess amount of dye could be washed away. This eliminated the background and resulted in a sufficiently high SNR for detection. However, acidification of SVs by addition of ATP induced only minor fluorescence changes and only in a small fraction of SVs, which argued against the suitability of Di-8ANEPPS for single SV measurements.

As the next option, a slow-response probe, Oxonal VI (bis-iosxazolone oxonol) whose magnitude of optical response is much larger than that of fast-response probes $(1 \%$ fluorescence change per mV) (Haugland, 2005), was tested. These probes are membrane permeable and their distribution across the membrane upon changes in membrane potential underlies their fluorescence response. Oxonol dyes have been often used for measuring the electrical gradient generated by the V-ATPase in SVs or in reconstituted systems in bulk assays (Goh et al., 2011; Hartinger and Jahn, 1993; Juge et al., 2006; Preobraschenski et al., 2014). However, this probe was not appropriate for the single vesicle assay. Because the probe was highly fluorescent in aqueous solution and a high background was observed after immobilization of the SVs in the presence of the dye, which in turn dramatically decreased the SNR. Next, VF2.1.Cl was tested. Unlike Oxonal VI, this probe inserts into the membrane of the SVs and therefore provided a low background after washing. In addition, it provided larger optical response compared to Di-8-ANEPPS due to its higher voltage-sensitivity (27\% per $100 \mathrm{mV}$ (Miller et al., 2012)).

In VF2.1Cl, dichlorosulfofluorescein, a membrane-impermeant fluorophore, connects to $\mathrm{N}$-dimethyl-aniline, an electron-rich quencher, via two units of $p$-phenylenevinylene as the molecular wire (Miller et al., 2012). As depicted in Figure 2-15A, once the inner leaflet of the SV membrane is more positively charged than the outer leaflet, the 
transmembrane electric field hinders electron transfer from the quencher to the excitedstate fluorophore, which in turn increases the fluorescence. VF2.1.Cl reports depolarization of the membrane by $27 \pm 1 \%$ fluorescence increases per $100 \mathrm{mV}$ (Miller et al., 2012). Upon immobilization of SVs in the presence of $100 \mathrm{nM} \mathrm{VF2.1.Cl,} \mathrm{followed} \mathrm{by}$ three washes, SVs were efficiently labeled with the dye and could be visualized using the $488 \mathrm{~nm}$ line of the argon laser for excitation (Figure 2-15B). Moreover, the dye showed reasonable photostability during a 20 s timeframe ( 10\% bleaching, Figure 2-15C). These data suggested that VF2.1.Cl was an appropriate probe for measuring electrical gradient in SVs at the single vesicle level.
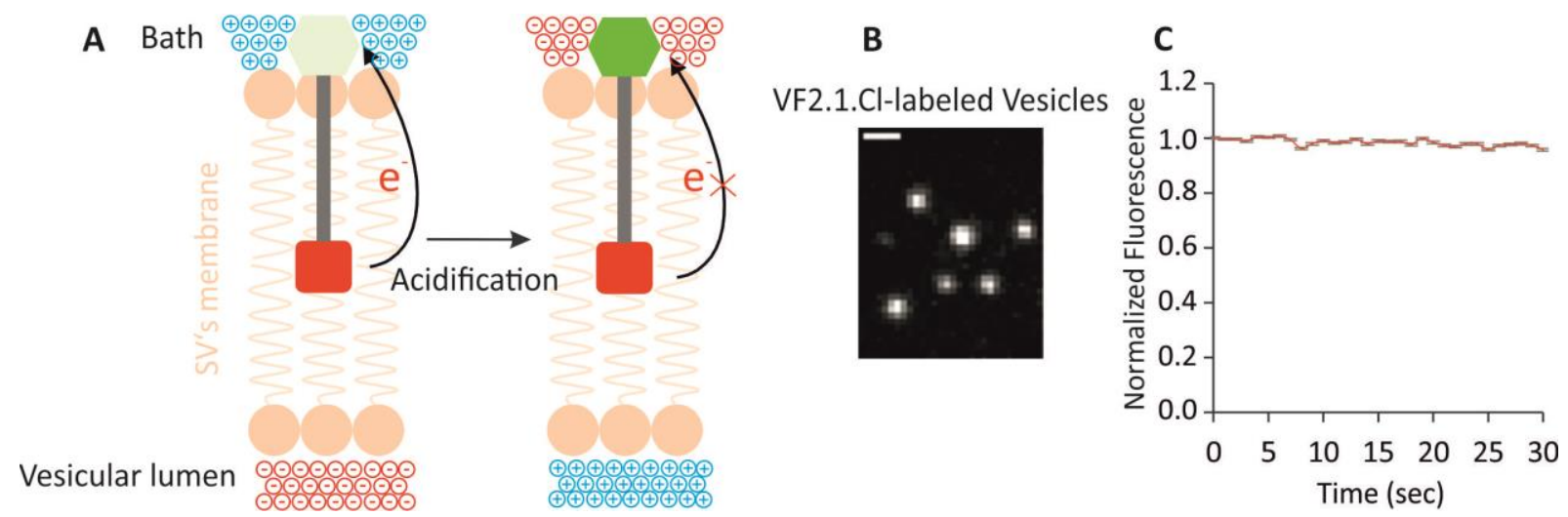

Figure 2-15 VF2.1.Cl as the appropriate potentiometric probe for single vesicle assay.

A) Schematic representation of mechanism of voltage sensing by VF2.1.Cl (adapted from (Miller et al., 2012)). When the inner leaflet of the SV membrane is more negatively charged than the outside, electron transfer from the electron-rich donor of $\mathrm{VF} 2.1 \mathrm{Cl}$ (red) to its fluorescent reporter (green) through the membrane-spanning molecular wire (gray) quenches the fluorescence (left panel). Upon luminal acidification, accumulation of protons in the lumen of SVs decreases the rate of electron transfer, which results in higher fluorescence (right panel). B) Representative fluorescent images of VF2.1.Cl-labled SVs. Vesicles were labeled by 1 hour incubation with $100 \mathrm{nM}$ of the dye in the bath solution. Excess amount of dye was removed before imaging. Labeled-SVs were excited by the $488 \mathrm{~nm}$ line of the argon laser and their emission was collected through a 515/30 nm filter. Scale bar is $1 \mu \mathrm{m}$. C) Trace of averaged integrated fluorescence of VF2.1.Cl labeled-SVs showing the extent of photobleaching over the experimental timeframe. The same laser and image acquisition settings as for the potentiometric experiments were used for photobleaching measurements. Error bars represent SEM of $\mathrm{n}$ single SVs, $\mathrm{n}=1,720$. 


\subsection{Single Vesicle Measurements}

As described in the previous section, super-ecliptic pHluorin and VF2.1.Cl displayed appropriate characteristics for measuring $\Delta \mathrm{pH}$ and $\Delta \psi$ in purified SVs, respectively. In this section, single vesicle measurements using these two probes are described.

\subsubsection{Single Vesicle Acidification Assay}

For $\mathrm{pH}$ measurements, spH-SVs were purified from transgenic mice as described in section 2.6.1 and immobilized on PLL-coated coverslips. SVs were continuously excited by the $488 \mathrm{~nm}$ line of the argon laser and their fluorescence was monitored by timelapse imaging at 5-10 Hz. In order to acidify vesicles, $\mathrm{P}^{3}$-(1-(2-nitrophenyl)-ethyl)-ester (NPE)-caged ATP (Table 2-4) was used. Upon UV flash photolysis, this compound releases ATP at rate constants ranging from 35 to $250 \mathrm{~s}^{-1}$ at physiological pH (Walker, 1988). The photolysis efficiency of NPE-ATP by a single UV pulse is estimated to be 80\% (McCray et al., 1980). However, depending on the light alignment in each setup, the uncaging efficiency can vary. In order to estimate the amount of free ATP released after a single UV flash in the setup used for this thesis, acidification experiments with both free ATP and NPE-ATP were performed, and the concentration of NPE-ATP and free ATP required to achieve the same luminal $\mathrm{pH}$ were correlated. This resulted in $\sim 60 \%$ uncaging efficiency. Accordingly, NPE-ATP concentrations were converted to free ATP concentrations using this measured uncaging efficiency.

In order to be able to study the regulatory effect of ions on the electrochemical gradient, the acidification assays were performed in glycine buffer, (Table 2-1) which was free of membrane-permeable ions. Therefore, it could be assumed that the contribution of ions other than protons to the acidification assays was negligible. However, it is known that GABA and glycine compete for vesicular uptake (Wojcik et al., 2006). Hence for experiments which involved GABA, K-gluconate buffer (Table 2-1) was used as the bath solution. In addition, due to optimal glutamate uptake at $4 \mathrm{mM}$ chloride (Schenck et al., 2009), the glycine buffer was supplemented with $4 \mathrm{mM} \mathrm{Cl}^{-}$as a tetraethylammonium (TEA) salt in measurements involving glutamate.

In all $\mathrm{pH}$ measurements, the initial luminal $\mathrm{pH}$ of SVs was assumed to be 7.4. To test this assumption, the luminal pH of SVs after purification was measured by $488 / 405 \mathrm{~nm}$ excitation ratiometric measurements (in collaboration with Dr. Andrew Woehler, Dep. 
Membrane Biophysics, MPIbpc, Göttingen, Germany). For these measurements, immobilized spH-SVs were illuminated with $405 \mathrm{~nm}$ and $488 \mathrm{~nm}$ lasers and their emission was collected through a 515/30 nm emission filter. Ratiometric values were obtained by dividing fluorescence collected with $488 \mathrm{~nm}$ excitation by that of $405 \mathrm{~nm}$ excitation. If the lumen of purified SVs were different than 7.4, a difference should have been observed between the ratiometric values before and after $10 \mu \mathrm{M}$ FCCP addition at pH 7.4. This was not the case. However, incubation of SVs at pH 5.5 in the presence of FCCP significantly decreased the ratiometric value (Figure 2-16). This indicates that SVs lose their proton contents during purification and reach an equilibrium with their surrounding solution.

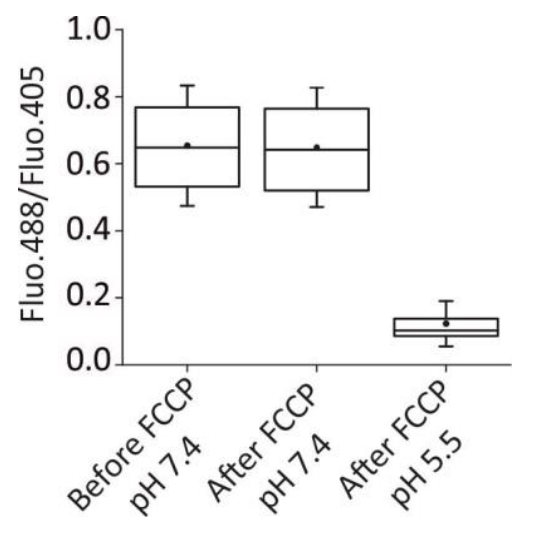

Figure 2-16 Measuring the initial pH of lumen of spH-SVs.

In order to check for the acidity of the lumen of SVs after the purification, immobilized spH-SVs were excited with 488 and $405 \mathrm{~nm}$ lasers and their fluorescence was collected in the absence and presence of FCCP at $\mathrm{pH} 7.4$ as well as $\mathrm{pH}$ 5.5. While at $\mathrm{pH} 5.5$ the fluorescence ratios significantly decreased, no differences were observed in the ratios before and after FCCP at pH 7.4. The top and bottom of the box plots are first and third quartiles, and the middle line and solid circle represent median and mean of the values, respectively. Whiskers indicate SD ( $\mathrm{n}=$ 143, 132 and 83 for 'Before FCCP pH7.4', 'After FCCP pH 7.4' and 'After FCCP pH 5.5', respectively).

\subsubsection{Single Vesicle Potentiometric Assay}

For potentiometric measurements, the SVs purified from wild type mouse brains were immobilized on PLL-coated coverslips in the presence of $100 \mathrm{nM} \mathrm{VF2.1.Cl.} \mathrm{The}$ labeled SVs were then washed to remove excess dye molecules. Similar as in $\mathrm{pH}$ measurements, glycine buffer was used as the bath solution. In case of measurements with GABA, glycine was substituted with K-gluconate, and for measurements with glutamate, 4 mM TEA.Cl was added to the bath solution. Labeled SVs were excited with 
the $488 \mathrm{~nm}$ line of the argon laser and imaged at $1 \mathrm{~Hz}$ with $200 \mathrm{~ms}$ exposure time to reduce photobleaching of the probe. Due to incompatibility of VF2.1.Cl with NPE-ATP and other tested caged-ATP compounds, SVs were acidified using Mg.ATP solution (Table 2-4).

\subsubsection{Antibody Labeling of Synaptic Vesicles}

To label immobilized SVs with antibodies, 30 min of blocking with 5\% (w/v) BSAcontaining phosphate saline buffer (PBS) was performed, followed by $30 \mathrm{~min}$ of incubation with antibody in blocking buffer. Coverslips were washed three times with PBS buffer before imaging. Additional fixation step (5 min with 4\% paraformaldehyde), permeabilization ( $5 \mathrm{~min}$ in Triton X-100 (0.1 \% v/v)), and incubation with secondary antibody (30 min in 5\% BSA in PBS) were included when required. In order to label SVs with antibody after each experiment, on-stage antibody staining was performed without changing the field of view by using the solution exchange system.

\subsubsection{Buffering Capacity Measurements}

In order to estimate the endogenous buffering capacity of SVs, the ammonia pulse technique was followed as described by (Maresova et al., 2010), with the modification that ammonia was substituted with methylamine. Methylamine is more effective than ammonia in dissipating $\mathrm{pH}$ gradients due to its lower $\mathrm{p} K_{\mathrm{b}}$ and higher membrane permeability (Ritchie and Gibson, 1987). To measure the buffering capacities at different luminal $\mathrm{pH}$ values, spH-SVs were first acidified with different NPE-ATP concentrations, and then alkalinized with $8 \mathrm{mM}$ methylamine. The $\mathrm{pH}$ of the methylamine solution was set to 7.4 to ensure that changes in fluorescence were not due to alkalinization of the bath solution. The fluorescence change induced by methylamine was first converted to $\Delta \mathrm{pH}$ using the fluorescence-pH calibration curve (Figure 2-12), and then converted to buffering capacity $(\beta)$ with Eq. 2.2 (Maresova et al., 2010):

$$
\beta=\frac{\Delta\left[\mathrm{CH}_{3} \mathrm{NH}_{3}^{+}\right]_{\text {luminal }}}{\Delta \mathrm{pH}} \quad \text { Eq.2.2 }
$$


where $\left[\mathrm{CH}_{3} \mathrm{NH}_{3}{ }^{+}\right]$luminal is the luminal concentration of protonated methylamine, and was calculated by Eq. 2.3 (Maresova et al., 2010):

$$
\left[\mathrm{CH}_{3} \mathrm{NH}_{3}{ }^{+}\right]_{\text {luminal }}=\frac{\left[\mathrm{CH}_{3} \mathrm{NH}_{2}\right]_{\text {total }} \times\left[\mathrm{H}^{+}\right]_{\text {luminal }}}{K_{\mathrm{a}}+\left[\mathrm{H}^{+}\right]_{\text {external }}}
$$

where $K_{\mathrm{a}}$ is the dissociation constant of methylamine and is equal $2.29 \times 10^{-11}$, $\left[\mathrm{H}^{+}\right]_{\text {luminal }}$ is the proton concentration in the lumen after methylamine application and $\left[\mathrm{H}^{+}\right]_{\text {external }}$ is the proton concentration in the bath solution.

\subsubsection{Proton Permeability Measurements}

In order to measure proton permeability, a previously published protocol (Budzinski et al., 2011) was followed with some modifications. Briefly, spH-SVs were equilibrated for 5 min in glycine/K-gluconate buffer at $\mathrm{pH} \mathrm{6.5,} \mathrm{after} \mathrm{which} \mathrm{the} \mathrm{bath} \mathrm{solution} \mathrm{was}$ exchanged with the same buffer at pH 7.4 (alkalinizing buffer), using the solutionexchange system. The fluorescence of spH-SVs was monitored in real-time by imaging at $5 \mathrm{~Hz}$. Almost complete fluorescence recovery was observed after solution exchange indicating that bath solution was efficiently exchanged. Bi-exponential fitting of individual fluorescence traces resulted in two components with the first reporting the proton efflux rate constant and the second accounting for photobleaching. Since the experiment was performed in a $\mathrm{pH}$ range where fluorescence and $\mathrm{pH}$ have a linear correlation, the time constant obtained from fitting was used to calculate the rate of $\mathrm{pH}$ change over time $(\Delta \mathrm{pH} / \mathrm{sec})$. The initial proton flux $(J)$ in $\mathrm{H}^{+} / \mathrm{sec}$ was then calculated using Eq. 2.4:

$$
J=\beta_{\mathrm{avg}} \times \Delta \mathrm{pH} / \mathrm{sec} \times \mathrm{Av} \times V_{\mathrm{Sv}} \times 10^{-3}
$$

where $\beta_{\text {avg }}$ is the averaged buffering capacity calculated from $\beta$ at pH 6.5 and at the luminal $\mathrm{pH}$ achieved after 1 second, Av is the Avogadro constant and $V_{\mathrm{SV}}$ is the volume of the SVs (Takamori et al., 2006). Next, proton permeability $\left(P_{\mathrm{H}^{+}}\right)$in $\mathrm{cm}_{\mathrm{sec}}{ }^{-1}$ was calculated by Eq. 2.5 (Grabe and Oster, 2001): 
$P_{\mathrm{H}^{+}}=J /\left(\operatorname{Av} \times S A \times \frac{V_{\mathrm{m}} F}{R T} \times \frac{\left[\mathrm{H}^{+}\right]_{\text {luminal }}-\left[\mathrm{H}^{+}\right]_{\text {external }} \exp \left(-V_{\mathrm{m}} F / R T\right)}{1-\exp \left(-V_{\mathrm{m}} F / R T\right)}\right)$

where $S A$ is the surface area of the SVs (5.4 x 10-15 $\mathrm{m}^{2}$, (Takamori et al., 2006)), $V_{\mathrm{m}}$ is the membrane potential (estimated from the potentiometric measurements with VF2.1.Cl), $\left[\mathrm{H}^{+}\right]_{\text {luminal }}$ and $\left[\mathrm{H}^{+}\right]_{\text {external }}$ are the concentration of protons at the luminal $\mathrm{pH}$ achieved after 1 second and at $\mathrm{pH} 7.4$, respectively, $F$ is the Faraday constant, $R$ is the universal gas constant, and $T$ is the absolute temperature.

\subsubsection{Data Analysis}

\subsubsection{Image Analysis of Single SVs}

Time series images were loaded as a 3D stack in MATLAB (Mathworks, Natick, MA). The average of the 10 initial frames in each image series was used for spot detection (Appendix 3). Single SVs were detected using a script based on a multiresolution algorithm (Olivo-Marin, 2002). A cutoff for appropriate size ( $<20$ pixels) and eccentricity $(<0.8$, defining 1 as a line) were applied to remove aggregated SVs from analysis. The script created sub-images centered on selected spots. The background for each SV was defined locally as the average intensity of neighboring pixels with the lowest intensity, and subtracted from the integrated intensity of the vesicles in each frame. Backgroundsubbed integrated intensities were normalized to values before chemical perturbations $\left(F_{\text {norm}}\right)$ and used for further calculation of $\mathrm{pH}$ and membrane potential.

\section{i. Image Analysis of spH-SVs}

For pH measurements, $F_{\text {norm }}$ of spH-SVs was converted to $\mathrm{pH}$ using the following equation derived from Eq. 2.1:

$$
\mathrm{pH}(t)=\mathrm{p} K_{\mathrm{a}}-\log _{10}\left[\frac{\left(1+10^{\mathrm{p} K_{a}-7.4}\right)-F_{\text {norm }}(t)}{F_{\text {norm }}(t)}\right]
$$

where $\mathrm{p} K_{\mathrm{a}}$ is equal to 7.2 and $\mathrm{pH}(t)$ and $F_{\text {norm }}(t)$ are the $\mathrm{pH}$ and $F_{\text {norm }}$ of a single spHSV at time $t$, respectively. 
Moreover, to measure the acidification kinetics of single SVs, the individual fluorescence traces were first converted to $\mathrm{pH}$ traces. This conversion was necessary due to the non-linearity between $\mathrm{pH}$ and fluorescence of $\mathrm{spH}$ at $\mathrm{pH}$ below 6.5. Biexponential fitting of $\mathrm{pH}$ traces resulted in two components with the first reporting the acidification rate constant and the second, which was in the range of 15 to 30 seconds, accounting for photobleaching.

\section{ii. $\quad$ Image Analysis of VF2.1.Cl-labeled SVs}

For potentiometric measurements, changes in membrane potential $(\Delta \psi)$ were calculated from $\mathrm{F}_{\text {norm }}$ of VF2.1.Cl labeled-SVs by:

$$
\Delta \psi=k_{\mathrm{VF2.1.Cl}} \times\left(F_{\text {norm }(\text { post })}-F_{\text {norm(pre) }}\right)
$$

where $k_{\mathrm{VF2} \text {.1.Cl }}$ is equal 370.37 and is the slope factor obtained from plotting fractional changes in fluorescence of VF2.1.Cl against the membrane potential, according to the voltage sensitivity of $27 \%$ per $100 \mathrm{mV}$ (Miller et al., 2012), and $F_{\text {norm(post) }}$ and $F_{\text {norm(pre) }}$ are $F_{\text {norm }}$ of single SVs pre and post chemical perturbations, respectively.

\section{iii. $\quad$ Image Analysis after Antibody Labeling}

In order to distinguish antibody-labeled from non-labeled SVs, individual SVs were detected as described above, in both the spH or VF2.1.Cl initial images and in images acquired after antibody labeling. Then $\mathrm{spH}$ or VF2.1.Cl-labeled spots whose center was within a $<2$ pixel proximity (pixel step was $150 \mathrm{~nm}$ ) of detected spots in antibody images were identified.

\subsubsection{Statistical Analysis}

In this study, bar graphs represent the mean of the SV population, and the error bars represent standard deviation (SD) from indicated number of independent experiments. In case of time constants and rates, mean \pm standard error of the mean (SEM) of the individual SVs with R-squared value of fitting $>0.7$, compiled from all the experimental replicates, is shown. The data from different vesicles in the same experiments are 
presented as two circles connected via a dashed line. Direct comparisons within the same experiment were performed using 2-sided Student's t-tests for paired samples. In other cases, Student's t-tests for unpaired samples were used. P-values are indicated in the figure legends. 


\section{Results}

\subsection{Optimized Single Vesicle Assay}

As described in section 2, different $\mathrm{pH}$-sensitive and potentiometric probes were tested for the single vesicle assays among which super-ecliptic pHluorin $(\mathrm{spH})$ and VF2.1.Cl showed the best characteristics for $\mathrm{pH}$ and potentiometric measurements, respectively. For pH measurements, SVs were purified from brains of $\mathrm{spH}-21$ transgenic mice using a protocol described by (Ahmed et al., 2013) with some modifications (see section 2.6.1). For potentiometric measurements, SVs purified from wild type mouse brain were labeled with $100 \mathrm{nM}$ of VF2.1.Cl. Measurements were performed using a TIRF setup, which was equipped with a UV flash lamp for NPE-ATP uncaging and a solution superfusion system for fast solution exchange (Figure 3-1).

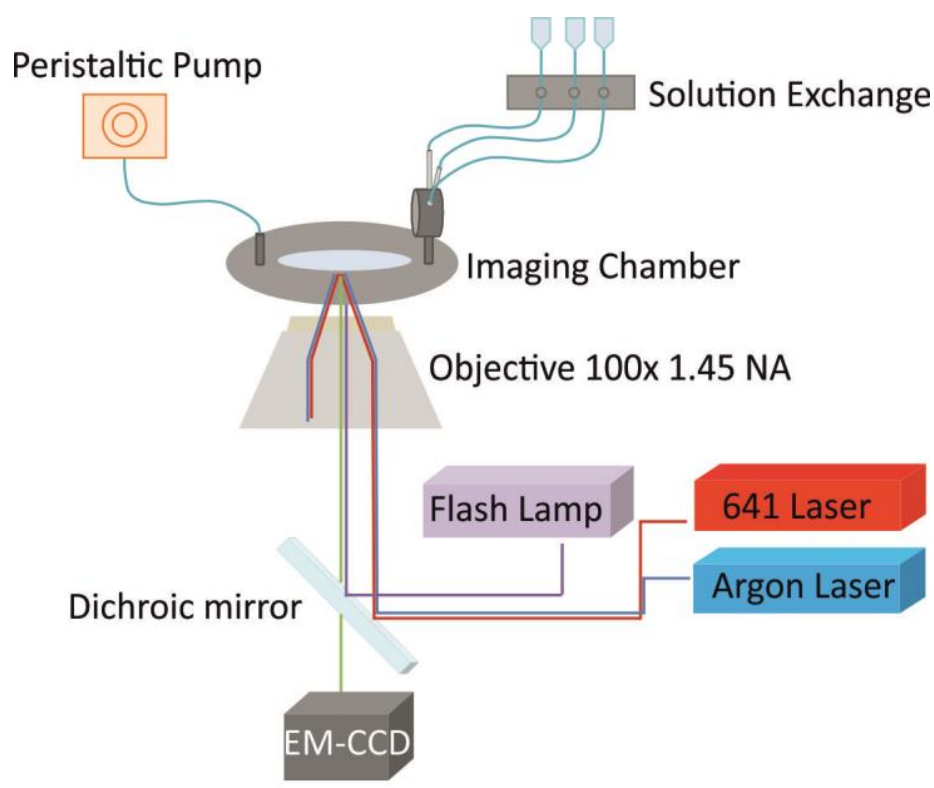

\section{Figure 3-1 Imaging setup for single vesicle imaging.}

spH/VF2.1Cl-labeled SVs were immobilized on PLL-coated glass coverslips, mounted in custom designed chambers with three inlets and one outlet, and imaged using a home-built multiwavelength TIRF microscope. The setup was equipped with a solution exchange system, a peristaltic pump and a flash lamp for NPE-ATP uncaging.

The final purified SV fraction was visualized by negative-staining EM (in collaboration with Dr. Dietmar Riedel, Laboratory of Electron Microscopy, MPIbpc, Göttingen, Germany), showing that the contribution of larger organelles to the final fraction was 
negligible (Figure 3-2A). In addition, to ensure that purified SVs were free of aggregates, immobilized vesicles were labeled with $1 \mu \mathrm{M}$ of the lipophilic carbocyanine dye DiD (Invitrogen). The integrated intensities of single particles were pooled into a histogram and could be fitted well with a lognormal distribution (Figure 3-2B), which corresponds to an intrinsic distribution of single particles when imaged in TIRF microscopy (Mutch 2007).
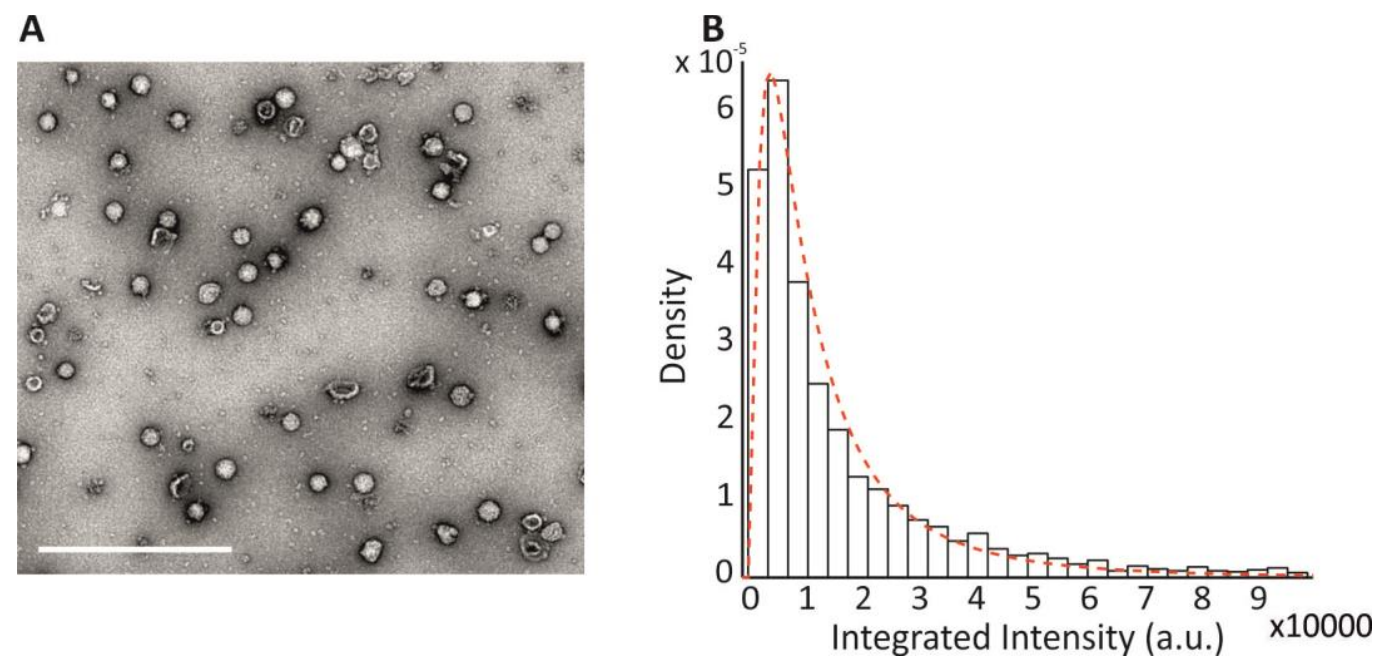

\section{Figure 3-2 Quality check of purified SV.}

A) Purified SVs were visualized by negative-staining EM, showing that the contribution of larger organelles to the final SV fraction was negligible. Scale bar is $500 \mathrm{~nm}$. B) Integrated intensity of DiD-labeled SVs were binned into a histogram which resulted in unimodal distribution, indicating that the SV sample is free of aggregates. Red line represent lognormal fit to the data.

In order to distinguish glutamatergic and GABAergic SVs, antibody labeling against their vesicular transporters was performed on-stage after $\Delta \mathrm{pH}$ or $\Delta \psi$ measurements, as described in section 2.7.3 (Figure 3-3A). More than 70\% of spH-SVs were labeled with antibody against VGLUT1 and $~ 20 \%$ of them were found GABAergic (Figure 3-3D). Since no difference was observed between the averaged response of VGLUT1-labeled SVs and those that were not labeled with an antibody against VGAT, the non-labeled SVs in case of VGAT staining were considered glutamatergic. Moreover, part of the V1 domain of the V-ATPase might dissociate during membrane purification, which was previously shown not to be a major concern (Takamori et al., 2006). In this thesis, the functionality of the V-ATPase of the purified SVs was assessed by calculating the percentage of spH-SVs which showed more fluorescence decrease upon NPE-ATP 
uncaging than can be attributed to photobleaching. With this analysis, more than $90 \%$ of both glutamatergic and GABAergic SVs were found functional (Figure 3-3E).

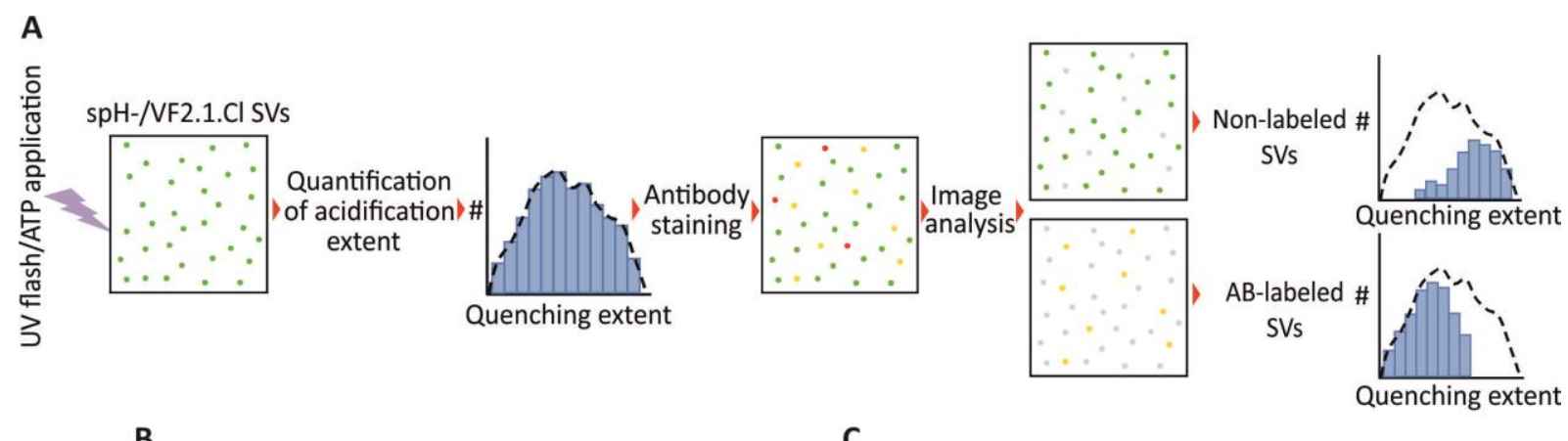

B

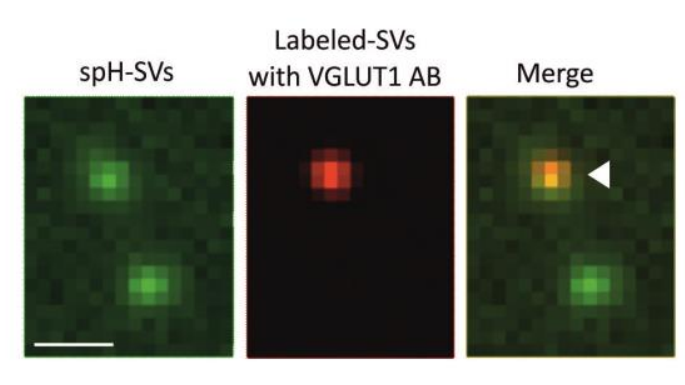

D

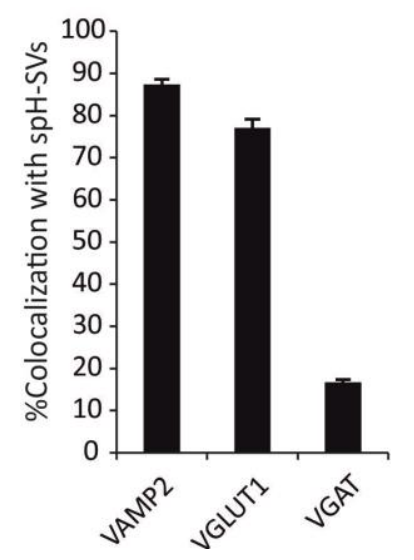

C

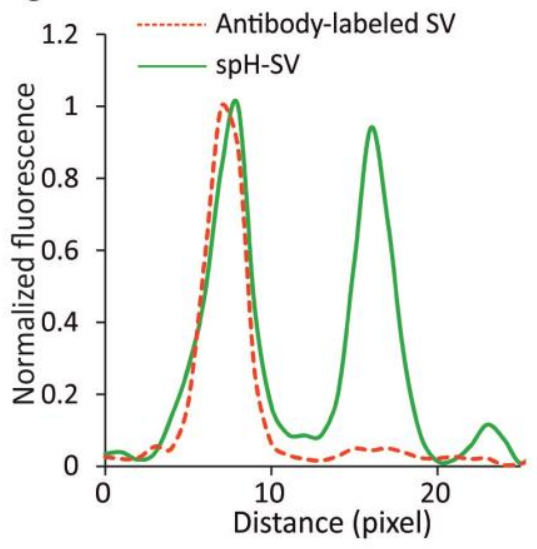

E

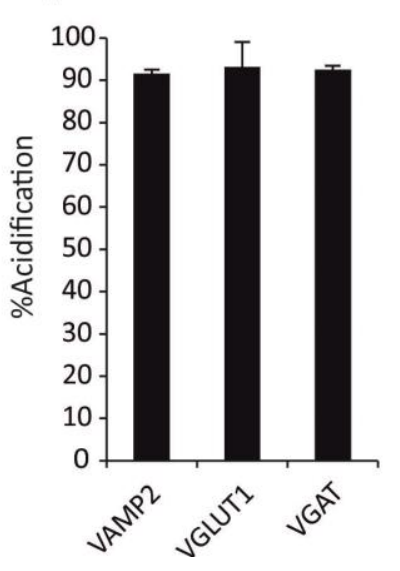

Figure 3-3 Distinguishing glutamatergic and GABAergic SVs by antibody staining.

A) After $\mathrm{pH}$ /potentiometric measurements, SVs were labeled with antibody against VGLUT1 or VGAT. In case of VGAT, unlabeled SVs were considered glutamatergic. Data analysis was first performed on the whole image and then the values for labeled and unlabeled SVs were separated. B) Representative images of spH-SVs, labeled SVs with antibody against VGLUT1 and the merged image, which shows that one of the spH-SVs was glutamatergic (shown by white arrow head). Scale bar is $1 \mu \mathrm{m}$. (C) Intensity cross section through spH-SVs from panel B. The pixel step was $150 \mathrm{~nm}$. (D) Almost 90\% of the spH-vesicles could be stained with antibodies raised against VAMP2. Since spH is tagged to luminal domain of VAMP2, this value represent the efficiency of antibody staining using the protocol described in section 2.7.3. More than 70\% of the spH-vesicles could be labeled with an antibody against VGLUT1 and $~ 20 \%$ with an antibody 
against VGAT. E) More than $90 \%$ of both glutamatergic and GABAergic purified spH-SVs acidified in the presence of ATP, which shows that V-ATPase remained functional during SV purification. Error bars in D and E represent SD of 3-5 experimental replicates.

\subsection{Electrochemical Gradient at different ATP Concentrations}

The luminal pH of single SVs after acidification with different concentrations of ATP was measured by collecting the fluorescence of spH-SVs upon photolysis of 1-5 mM NPE-ATP in glycine buffer (Table 2-1). NPE-ATP uncaging led to fluorescence quenching of spH-SVs in a dose-dependent manner (Figure 3-4A). For each acidification measurement, a photobleaching image was acquired by measuring the fluorescence of spH-SVs with the same experimental settings used for $\mathrm{pH}$ measurements in the absence of NPE-ATP. In order to estimate the steady-state luminal pH at each ATP concentration, fluorescence traces were first corrected for photobleaching by dividing individual traces by the averaged fluorescence trace obtained from the corresponding photobleaching images. Corrected fluorescence traces were then converted to $\mathrm{pH}$ traces using the $\mathrm{pH}$ fluorescence calibration curve (Figure 2-12). Luminal pH at each ATP concentration was then calculated from the individual $\mathrm{pH}$ traces as the averaged $\mathrm{pH}$ values between 15 to 20 seconds after NPE-ATP uncaging. The averaged $\mathrm{pH}$ values from 3-5 experimental replicates were then plotted against ATP concentrations, calculated from NPE-ATP with an uncaging efficiency of $60 \%$ (see section 2.7.1) (Figure 3-4B). These data show that at saturating concentrations of ATP $(2-3 \mathrm{mM})$ in a buffer which was free of membranepermeable ions, the interior of SVs acidifies to a pH of $\sim 6.57 \pm 0.04$ (SD).

Moreover, the acidification kinetics of single vesicles were obtained at each ATP concentration by a bi-exponential fit to individual $\mathrm{pH}$ traces as described in section 2.7.6.1. These data show that a faster rate of acidification is achieved when ATP is in abundant supply. The averaged acidification rate constants $(1 / \mathrm{sec})$ were plotted against the concentration of ATP, and fit with the Michaelis-Menten equation (Figure 3-4C). This resulted in a maximum acidification rate constant of $1.78 \pm 0.061 / \mathrm{sec}( \pm \mathrm{SEM})$ and $K_{\mathrm{m}}$ of $0.63 \pm 0.12 \mathrm{mM}$ ATP. 

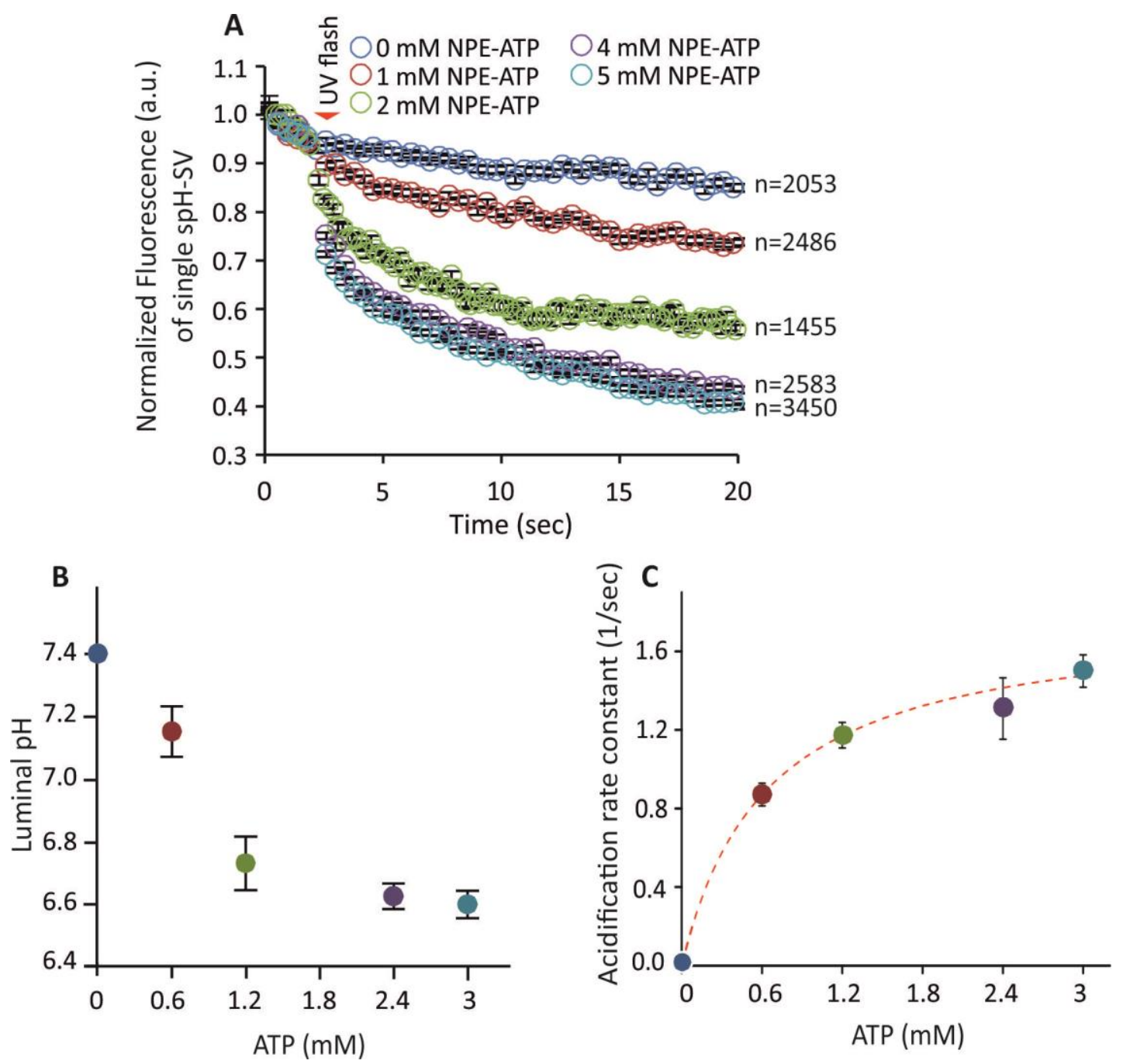

Figure 3-4 Luminal pH of single spH-SVs after acidification at different ATP concentrations.

A) Averaged fluorescence traces of spH-SVs at different NPE-ATP (data from 3-5 independent experimental replicates are compiled for the averaged trace). UV flash indicates where ATP is released by photolysis. The trace in the absence of ATP shows photobleaching of the probe over the experimental timescale. Error bars represent SEM of $n$ single SVs ( $\mathrm{n}$ is indicated on the traces). B) The averaged luminal pH of spH-SVs at different free ATP (calculated based on $60 \%$ uncaging efficiency). Error bars represent SD of 3-5 experimental replicates. C) Acidification rate constants of single spH SVs at different ATP concentrations; red line shows Michaelis-Menten fit to the data (R-Square $=0.99, K_{\mathrm{m}}=0.63 \pm 0.12 \mathrm{mM} \mathrm{ATP}, V_{\max }=1.78 \pm 0.061 / \mathrm{sec}$, Error bars represent SEM of $\mathrm{n}$ single SVs; $\mathrm{n}=112,128,43$ and 69 for 0.6, 1.2, 2.4 and $3 \mathrm{mM}$ ATP, respectively).

Next, the membrane potential generated across the membrane of single SVs upon acidification at different ATP concentrations was measured by labeling SVs with $100 \mathrm{nM}$ of VF2.1.Cl in glycine buffer. In agreement with the $\mathrm{pH}$ measurements, addition of 0.6-3 mM ATP to labeled SVs induced an increase in VF2.1.Cl fluorescence in a dose dependent manner (Figure 3-5A). The averaged fluorescence values at 15-20 seconds after ATP 
application were converted to changes in membrane potential by Eq. 2.7. The results from 3-5 experimental replicates for each ATP concentration were averaged and plotted against ATP concentrations (Figure 3-5B). These results show that upon acidification of SVs with $3 \mathrm{mM} \mathrm{ATP}$, a $\Delta \psi$ of $82.97 \pm 16.9 \mathrm{mV}( \pm \mathrm{SD})$ is formed across the membrane of vesicles.
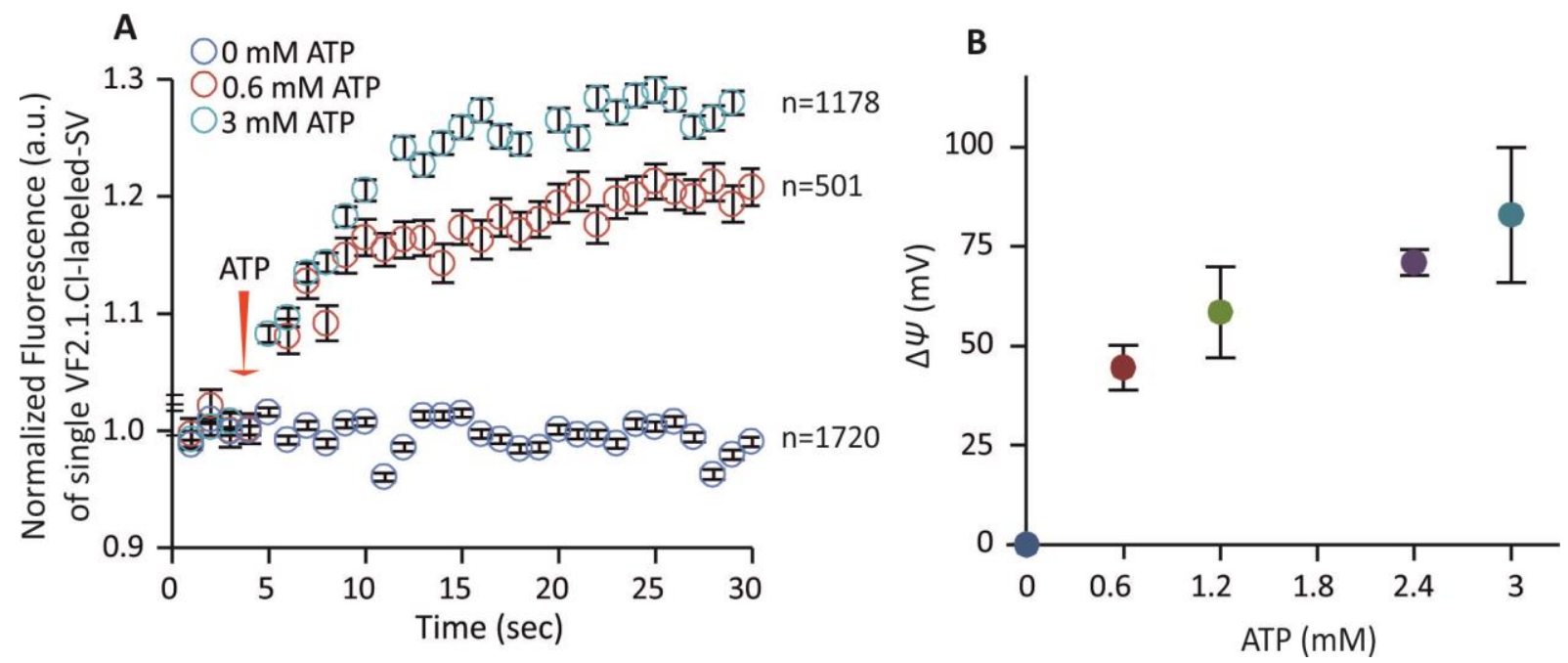

Figure 3-5 Changes in membrane potential across the membrane upon acidification at different ATP.

A) Averaged fluorescence traces of VF2.1.Cl-labled SVs in response to addition of different Mg.ATP to the bath solution (data from 3-5 independent experimental replicates are compiled for the averaged trace). The trace in the absence of ATP shows photobleaching of the probe over experimental timescale. Error bars represent SEM of $\mathrm{n}$ single SVs ( $\mathrm{n}$ is indicated on the traces). B) Averaged extent of change in membrane potential upon ATP-induced acidification. Error bars represent SD of 3-5 experimental replicates.

Interestingly, when $\Delta \mathrm{pH}$ and $\Delta \psi$, measured at the same concentration of ATP, were plotted against each other, a non-linear relationship was observed (Figure 3-6). 


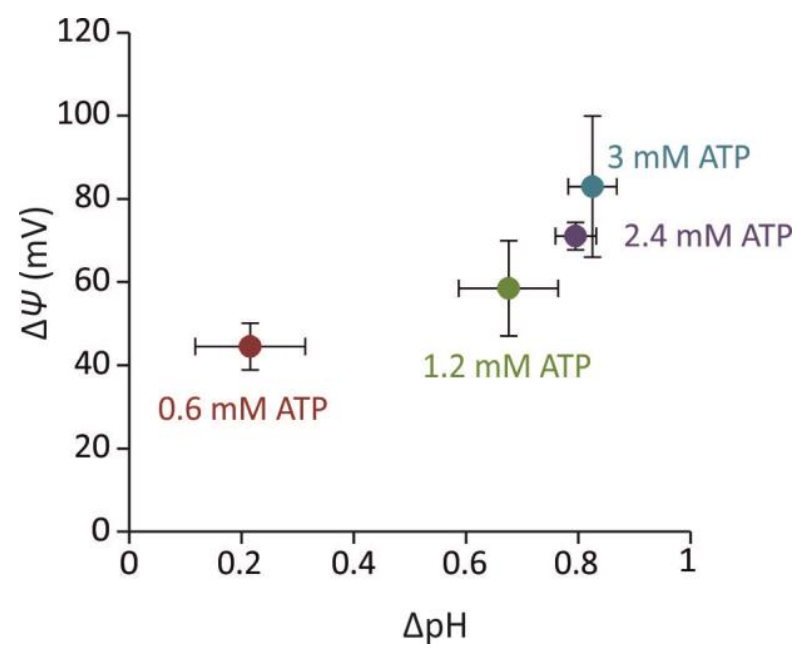

Figure 3-6 Relationship between $\Delta \mathrm{pH}$ and $\Delta \psi$.

Changes in the membrane potential upon acidification with different concentrations of ATP are plotted against the $\mathrm{pH}$ gradient induced by the corresponding concentration of ATP. It should be noted that the $\mathrm{pH}$ and potentiometric measurements were performed separately and the absolute values must be viewed cautiously.

\subsection{1 $\Delta p H$ and $\triangle \psi$ in Glutamatergic and GABAergic SVs}

After each $\mathrm{pH}$ or potentiometric measurement, on-stage antibody staining against VGAT was performed using the solution exchange system of the TIRF setup. This allowed for the detection of glutamatergic and GABAergic SVs in the same population of vesicles. Surprisingly, when the luminal $\mathrm{pH}$ of GABAergic and glutamatergic SVs were compared, a significantly lower luminal $\mathrm{pH}$ was observed in glutamatergic compared to GABAergic SVs. The same results were obtained at all tested ATP concentrations (0.6-3 $\mathrm{mM}$ ) (Figure 3-7A). Consistent with these data, significantly larger $\Delta \psi$ was also measured in glutamatergic compared to GABAergic SVs in each potentiometric measurement (Figure 3-7B). However, no significant difference was observed between acidification rate constants of these SVs (Figure 3-7C), suggesting no difference in the force driving protons into the vesicular lumen. Thus, to unravel the underlying mechanism for the observed difference, two other factors regulating $\Delta \mu_{\mathrm{H}+}$ were measured in these SVs: buffering capacity and proton permeability. 

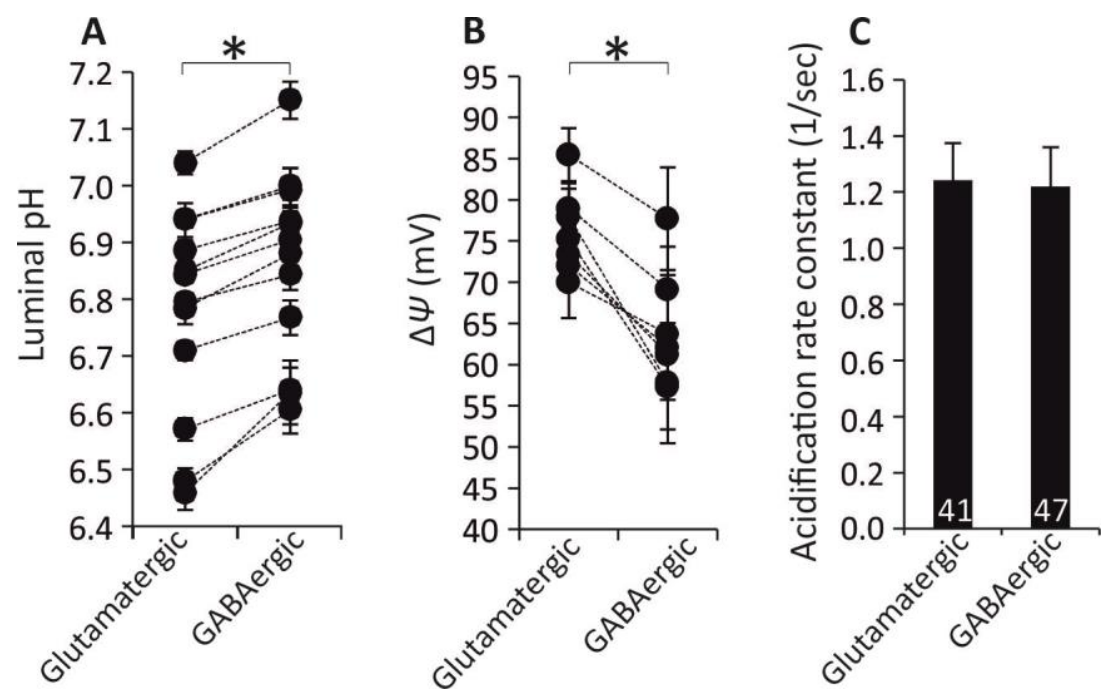

Figure 3-7 Comparison between glutamatergic and GABAergic vesicles in their proton electrochemical gradient.

A) Luminal $\mathrm{pH}$ of glutamatergic and GABAergic SVs after acidification with different concentrations of ATP. Glutamatergic SVs reached $0.1 \pm 0.03( \pm$ SD) pH units lower luminal pH compared to GABAergic SVs. B) Changes in membrane potential across the membrane of glutamatergic and GABAergic SVs upon acidification with different ATP concentrations. The magnitude of the membrane potential was $11.99 \pm 5.2 \mathrm{mV}( \pm \mathrm{SD})$ larger in glutamatergic compared to GABAergic SVs. Two circles connected via a dashed line represent the average response of glutamatergic and GABAergic SVs from the same experiment. Error bars represent SEM of single SVs. Number of glutamatergic and GABAergic SVs per measurement was on average $444 \pm 122$ and $160 \pm 78( \pm \mathrm{SD})$, respectively. p-value $=2.7 \times 10^{-5}$ and $8.7 \times 10^{-4}$ in $\mathrm{A}$ and $\mathrm{B}$, respectively. C) Acidification rate constant of glutamatergic and GABAergic SVs at $2.4 \mathrm{mM}$ ATP. Averaged acidification rate constants $( \pm$ SEM) were $1.24 \pm 0.13(1 / \mathrm{sec})$ and $1.22 \pm 0.14$ $(1 / \mathrm{sec})$ for the glutamatergic and GABAergic SVs, respectively. Error bars represent SEM of $n$ single SVs ( $\mathrm{n}$ is indicated on the bars).

\subsection{Buffering Capacity of Single SVs}

A conventional approach to measure buffering capacity is to measure the $\mathrm{pH}$ changes in a system accompanied by addition of known amounts of acid or base (Goldsmith and Hilton, 1992). Accordingly, as described in section 2.7.4, spH-SVs were acidified to different luminal $\mathrm{pH}$ values and the buffering capacity in units of $\mathrm{mM} / \Delta \mathrm{pH}$ was estimated by measuring the $\mathrm{pH}$ changes induced by addition of a constant concentration of methylamine, a weak permeable base (Figure 3-8). 


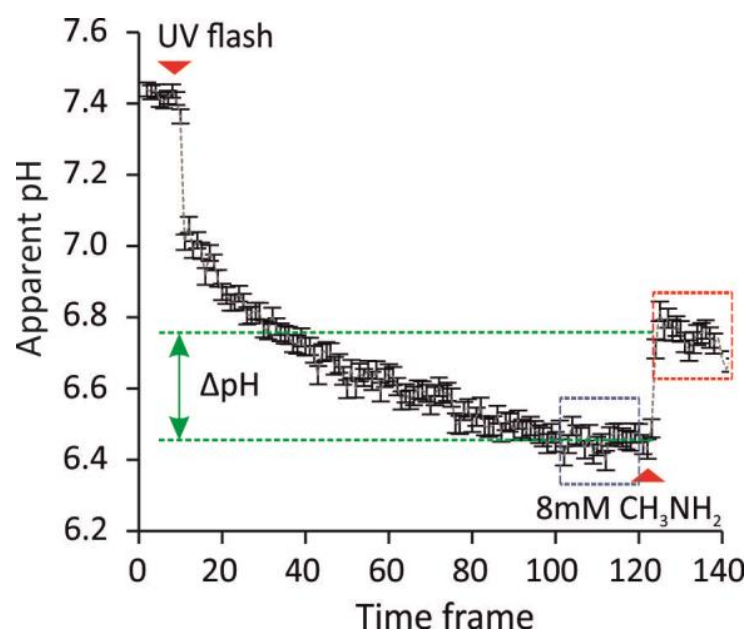

Figure 3-8 Buffering capacity measurement.

The average response of single spH-SVs to uncaging of $4 \mathrm{mM} \mathrm{NPE-ATP}$ and subsequent addition of $8 \mathrm{mM}$ methylamine. The internal concentration of protonated methylamine $\left(\left[\mathrm{CH}_{3} \mathrm{NH}_{3}{ }^{+}\right]_{\text {luminal }}\right)$ was obtained according to Eq. 2.3 for which $\left[\mathrm{H}^{+}\right]_{\text {luminal }}$ was calculated from the averaged $\mathrm{pH}$ values of frames after methylamine application (red box). The resulting $\left[\mathrm{CH}_{3} \mathrm{NH}_{3}{ }^{+}\right]$luminal was divided by $\Delta \mathrm{pH}$ induced by methylamine (green dashed lines and arrows) to calculate the buffering capacity of the SVs (Eq. 2.2). The buffering capacities were then plotted in Figure 3-9 against the average $\mathrm{pH}$ values of frames before methylamine application (blue box). Error bars indicate SEM of $\mathrm{n}$ single SVs, $\mathrm{n}=832$.

As shown in Figure 3-9, the buffering capacity was different at varying luminal $\mathrm{pH}$ values. Higher buffering capacity was measured at lower luminal $\mathrm{pH}$, and a linear correlation was observed between $\mathrm{pH}$ and $\beta$.

The buffering capacity $(\beta)$ determines the relative contribution of a luminal proton to $\Delta \mathrm{pH}$, while the contribution to $\Delta \psi$ is constant. If the buffering capacity in glutamatergic and GABAergic vesicle was differently regulated over $\mathrm{pH}$, this could perhaps explain the measured difference in their $\mathrm{pH}$ gradient. However, no difference was observed between buffering capacity of glutamatergic and GABAergic SVs tested between pH $\sim 6.7-7.1$ (Figure 3-9 inset). 


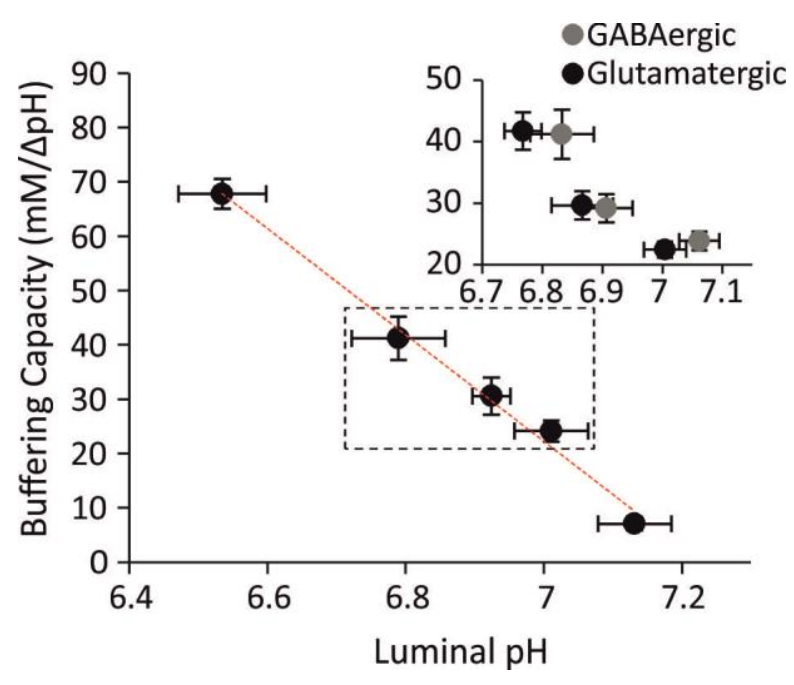

Figure 3-9 Relationship between buffering capacity and luminal pH.

Averaged buffering capacity of single SVs was plotted against the luminal $\mathrm{pH}$ at which the measurement was performed. Red line shows linear correlation between $\beta$ and luminal $\mathrm{pH}$ over the tested $\mathrm{pH}$ range $(\mathrm{R}-\mathrm{Square}=0.99, \mathrm{y}=-97.896+707.48)$. At three luminal $\mathrm{pH}$ values, inside the dashed box, the values for glutamatergic and GABAergic SVs were individually determined. No difference in buffering capacity was observed between these two vesicle subclasses in their endogenous buffering capacities. Both horizontal and vertical error bars represent SD of 3-5 experimental replicates.

\subsection{Proton Permeability of Single SVs}

A steady state electrochemical gradient is achieved when the rate of proton import through the V-ATPase reaches equilibrium with proton efflux from the vesicle. Differences in proton leakage rates have been shown to result in variations in luminal pH in different organelles (Van Dyke, 1993). Therefore, it was tested whether such a mechanism would be responsible for the observed differences in the electrochemical gradient of glutamatergic and GABAergic SVs. For this purpose, spH-SVs were first equilibrated with glycine buffer at $\mathrm{pH}$ 6.5, leading to quenching of the spH fluorescence. Then the proton efflux time constant $\left(\tau_{\text {efflux }}\right)$ was measured by monitoring the fluorescence recovery of spH-SVs after fast exchange of the bath solution to glycine buffer, pH 7.4 (see section 2.7.5). On average, spH-SVs reached their maximum fluorescence at $\mathrm{pH} 7.4$ within 4-5 seconds (Figure 3-10). The proton permeability $\left(P_{\mathrm{H}+}\right)$ was then calculated based on the measured $\tau_{\text {efflux }}$ using Eq. 2.4 and 2.5. 

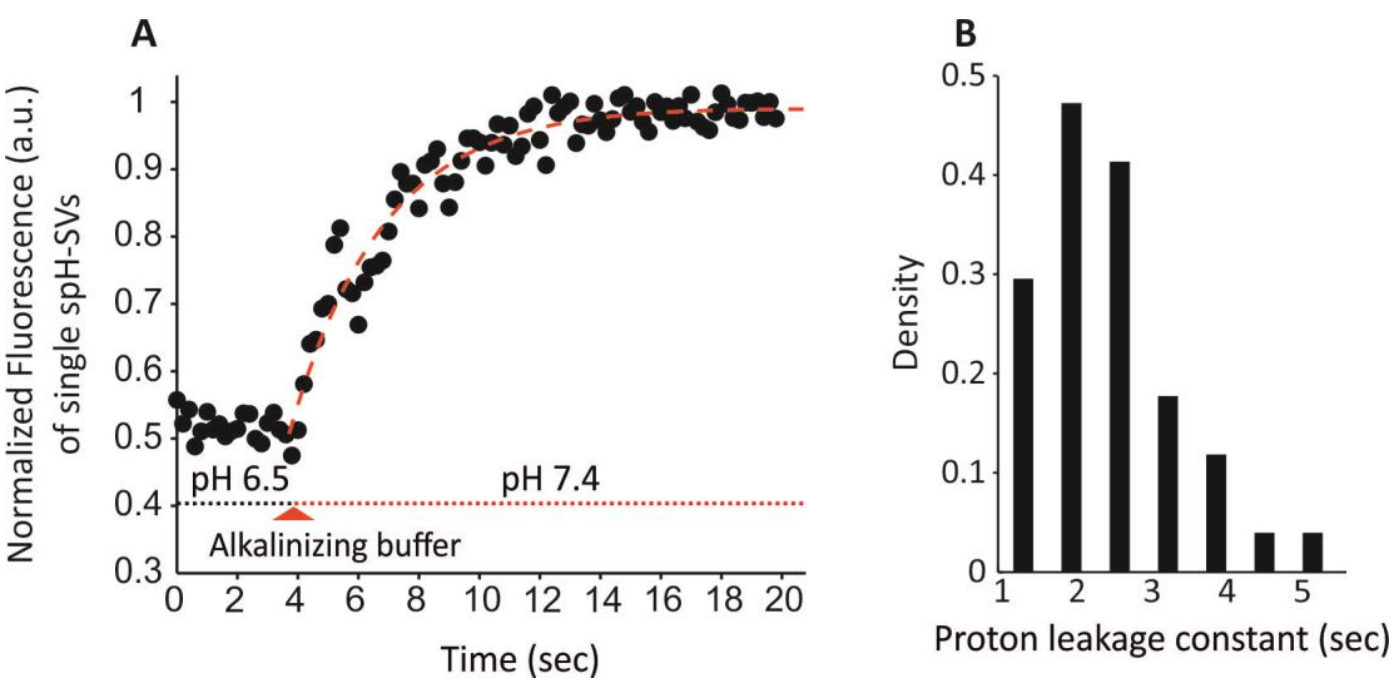

\section{Figure 3-10 Proton efflux measurements.}

A) As described in section 2.7.5, spH-SVs were first equilibrated with buffer, $\mathrm{pH} 6.5$, and then the bath solution was exchanged rapidly with the same buffer, but now at $\mathrm{pH} 7.4$ (Alkalinizing buffer). Averaged fluorescence trace of single spH-SVs in response to $\mathrm{pH}$ change in the bath solution. Red line shows a bi-exponential fit to the fluorescence recovery. B) Proton efflux constant values obtained from 3 independent experiments were pooled and binned into a histogram. The averaged $\tau_{\text {efflux }}$ from the whole population was calculated as $2.44 \pm 0.93 \mathrm{sec}( \pm$ SD).

Intriguingly, upon comparison of glutamatergic and GABAergic SVs, a significantly lower $\tau_{\text {efflux }}$ was observed in GABAergic SVs, indicating a faster efflux rate (Figure 3-11). Accordingly, a higher proton permeability was calculated in GABAergic SVs $\left(P_{\mathrm{H}^{+}}=15.2 \mathrm{x}\right.$ $10^{-3}$ and $13.5 \times 10^{-3} \mathrm{~cm} \mathrm{sec}^{-1}$ in GABAergic and glutamatergic SVs, respectively, calculated based on Eq. 2.4 and 2.5). 


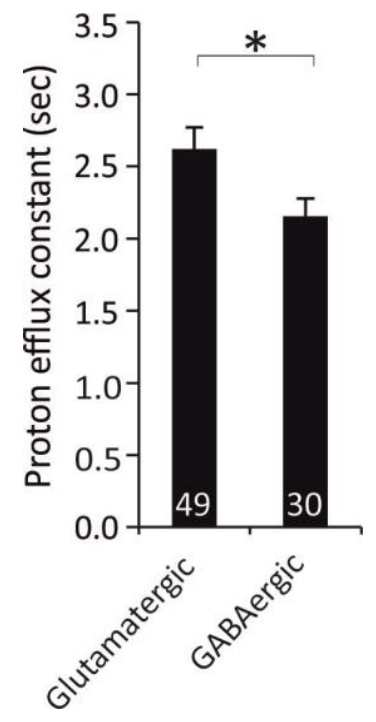

Figure 3-11 Comparison between glutamatergic and GABAergic SVs in proton permeability.

The average proton efflux constant was significantly smaller in GABAergic SVs, indicating that these vesicles have a higher proton permeability compared to glutamatergic SVs. Error bars represent SEM of $\mathrm{n}$ single SVs ( $\mathrm{n}$ is indicated on the bars). $\mathrm{p}$-value $=0.03$. Averaged proton efflux constants in seconds $( \pm$ SEM) were $2.62 \pm 0.15$ and $2.15 \pm 0.12$ for the glutamatergic and GABAergic SVs, respectively.

The proton efflux from the acidified lumen of vesicles can occur through either the lipid bilayer or through transmembrane proteins (Wan et al., 2002). The total flux of protons through the lipid bilayer is directly proportional to the surface area of the vesicles assuming an identical lipid composition. If the differences in $P_{[\mathrm{H}+]}$ were due to different vesicle size, GABAergic SVs would need to be $\sim 9 \mathrm{~nm}$ larger in diameter than glutamatergic vesicles (calculated based on Eq. 2.4 and 2.5). To test if this was the case, the diameter of glutamatergic and GABAergic SVs $\left(d_{\mathrm{SV}}\right)$ was measured by electron microscopy (in collaboration with Dr. Dietmar Riedel, Laboratory of Electron Microscopy, MPIbpc, Göttingen, Germany). For this purpose, immunogold labeling against VGAT was performed on SVs purified from wild type mouse brain (Figure 3-12). Reasonably assuming that non-labeled SVs are predominantly glutamatergic, no significant difference was observed between the average diameter $\left(d_{S V}\right)$ of labeled SVs (GABAergic) and non-labeled vesicles $\left(d_{\mathrm{SV}}=45.5 \pm 8.1 \mathrm{~nm}\right.$ and $45.8 \pm 10.3 \mathrm{~nm}$ for glutamatergic and GABAergic SVs, respectively, which is close to previously determined $d_{\text {Sv }}$ (Takamori 2006)). 
A
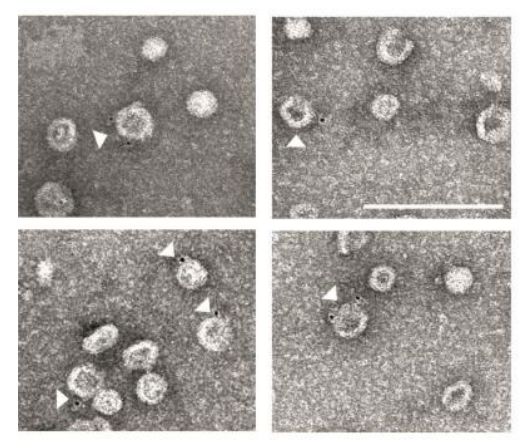
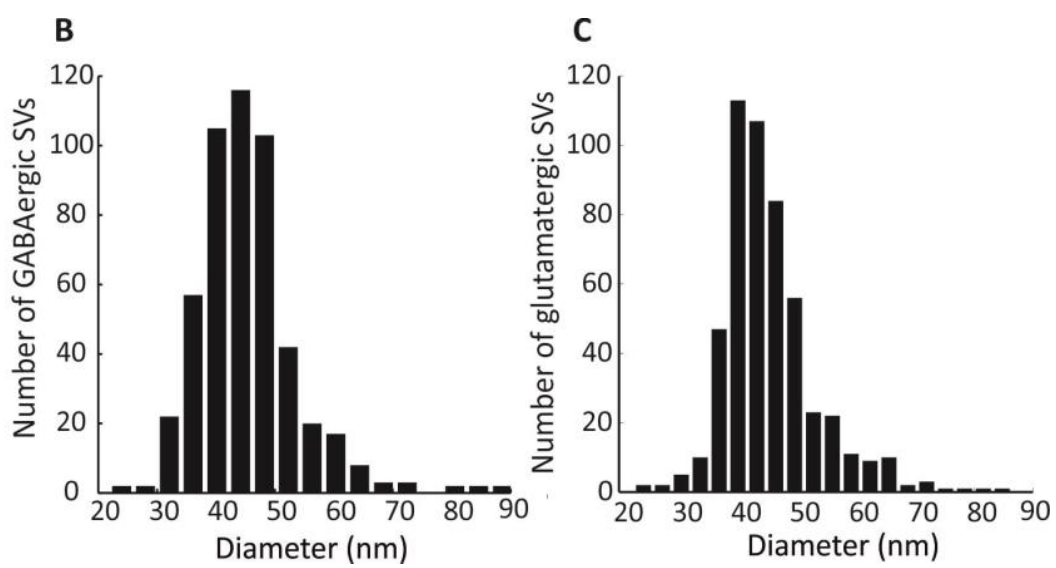

Figure 3-12 Size distribution of glutamatergic and GABAergic SVs.

A) Representative EM pictures of immunogold labeled SVs. Purified SVs from mouse brain were labeled with antibody against VGAT (131 004, Table 2-5). White arrow heads indicate labeled SVs which are GABAergic. Non-labeled SVs were considered glutamatergic. Scale bar is $200 \mathrm{~nm}$. The diameter for individual vesicles was calculated from two measurements orthogonal to each other. All the values for labeled (GABAergic, $n=510$ ) (B) and non-labeled vesicles (glutamatergic, $\mathrm{n}=510$ ) (C) were pooled and binned into histograms. Averaged diameters $( \pm$ SD) were $45.8 \pm 10.3 \mathrm{~nm}$ for the GABAergic and $45.5 \pm 8.1 \mathrm{~nm}$ for the glutamatergic vesicles.

These results suggest that a protein mediated mechanism is responsible for the higher proton permeability in GABAergic SVs. However, quantitative mass spectrometry analysis of proteins in glutamatergic and GABAergic SVs has revealed that these vesicles share the bulk of their constituents and the vesicular transporters are the only proteins that are exclusively present in one of these SV subclasses (Gronborg et al., 2010). Therefore, it would be reasonable to attribute the additional proton permeability in GABAergic SVs to VGAT. In line with this view, a positive correlation was observed between the luminal $\mathrm{pH}$ of acidified GABAergic SVs with the intensity of antibody against VGAT (Figure 3-13). Since a higher labeling intensity would be expected to correlate with a higher copy number of the transporter, these data imply that SVs with more VGAT have a greater proton permeability and thus a higher $\mathrm{pH}$. 


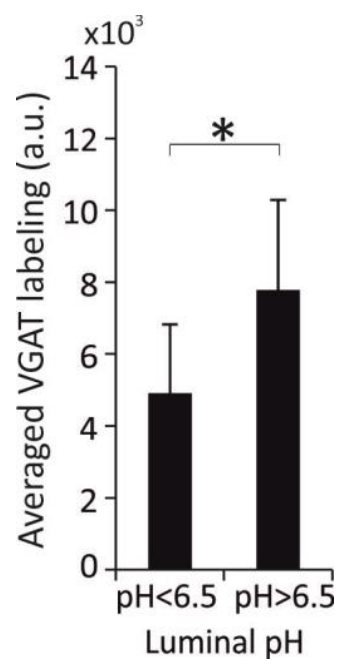

Figure 3-13 Correlation between antibody-labeling intensity and luminal pH in GABAergic SVs.

After acidification of spH-SVs with $2.4 \mathrm{mM}$ ATP in K-gluconate buffer, GABAergic SVs were labeled with an antibody against VGAT (131 103C5, Table 2-5). Then the correlation between their luminal $\mathrm{pH}$ and antibody-labeling intensity was analyzed for each SV. Interestingly, a significantly higher antibody labeling was observed in GABAergic SVs whose luminal pH was higher than 6.5 (the averaged luminal $\mathrm{pH}$ of GABAergic SVs was $6.53 \pm 0.07$ ). Error bars represent $\mathrm{SD}$ of 7 independent experiments. $\mathrm{p}$-value $=0.03$.

The finding that VGAT is permeable to protons opened the possibility of testing the controversial hypothesis that VGAT functions as a GABA/ $\mathrm{H}^{+}$antiporter (Ahnert-Hilger and Jahn, 2011). If this were the case, the apparent proton efflux from GABAergic SVs would be enhanced in the presence of GABA. To test this notion, the proton permeability of GABAergic SVs was measured in K-gluconate buffer in the absence and presence of 10 mM GABA. Indeed, applying an alkalinizing buffer containing $10 \mathrm{mM}$ GABA significantly accelerated the proton efflux from GABAergic vesicles (Figure 3-14), demonstrating that VGAT does in fact function as a GABA/ $\mathrm{H}^{+}$exchanger. 


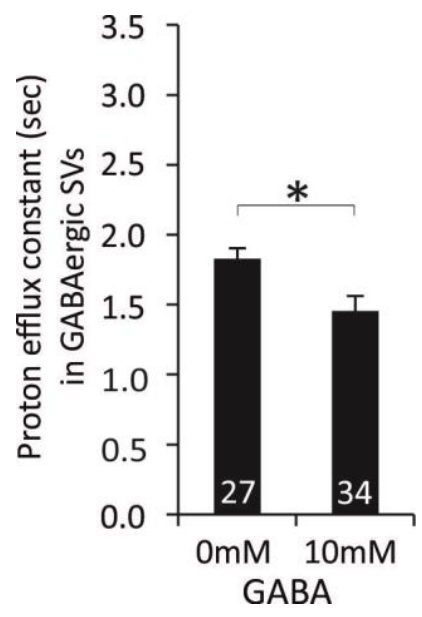

Figure 3-14 Effect of GABA on proton efflux from GABAergic SVs.

In order to test a GABA/ $\mathrm{H}^{+}$antiport mechanism by VGAT, the effect of GABA on the proton efflux time constant was measured. Including $10 \mathrm{mM}$ GABA in the alkalinizing buffer (see Figure 3-10 and section 2.7.5), significantly decreased $\tau_{\text {efflux }}$ in GABAergic SVs, indicating that GABA is indeed coupled to proton efflux. Error bars represent SEM of $\mathrm{n}$ single SVs (n is indicated on the bars). $\mathrm{p}$ value $=0.03$. Averaged proton efflux constants in seconds $( \pm$ SEM) were $1.83 \pm 0.07$ and $1.45 \pm$ 0.10 in the absence and presence of GABA, respectively. The decrease in efflux time constant in the absence of GABA compared to the measurements in glycine buffer (compare with Figure 3-11) is most likely due to high $\mathrm{K}^{+}$in K-gluconate buffer.

\subsection{Effect of Regulatory Ions on the Electrochemical Gradient}

Ionic conductances in an organelle are crucial regulatory elements of both the formation rate and the extent of the electrochemical gradient (Grabe and Oster, 2001). Different regulatory ions, depending on their charge and stoichiometry, can modulate the electrochemical gradient in favor of one or both of its components. During neuronal activity, sodium, potassium and chloride play substantial roles in different steps of SV recycling. More specifically, there is evidence that changes in the presynaptic concentrations or the function of the responsible transporters of $\mathrm{Na}^{+}$(Huang and Trussell, 2014), $\mathrm{K}^{+}$(Goh et al., 2011) or $\mathrm{Cl}^{-}$(Riazanski et al., 2011) can significantly affect the neurotransmitter filling into the vesicles. However, there is a scarcity of detail regarding their effect on the two components of the electrochemical gradient. In this thesis, the effect of these ionic species on both $\Delta \mathrm{pH}$ and $\Delta \psi$ was measured. Moreover, in order to determine whether these regulatory ions help to achieve an optimal $\Delta \mathrm{pH}$ and $\Delta \psi$ balance for loading of glutamatergic and GABAergic SVs, the responses of these vesicle populations to $\mathrm{Na}^{+}, \mathrm{K}^{+}$and $\mathrm{Cl}^{-}$were compared. 


\subsubsection{Effect of Sodium and Potassium on $\Delta \mu_{H+}$}

In order to assess the effect of $\mathrm{Na}^{+}$and $\mathrm{K}^{+}$on the $\mathrm{pH}$ gradient, spH-SVs were first acidified with $1 \mathrm{mM}$ of Mg-ATP. Thereafter, $\mathrm{Na}^{+}$or $\mathrm{K}^{+}$as gluconate salts were added to the bath solution (Figure 3-15A and C). This led to an increase in the fluorescence of spH-SVs, indicating that the lumen of acidified SVs reached a significantly higher $\mathrm{pH}$ (Figure 3-15B and D).
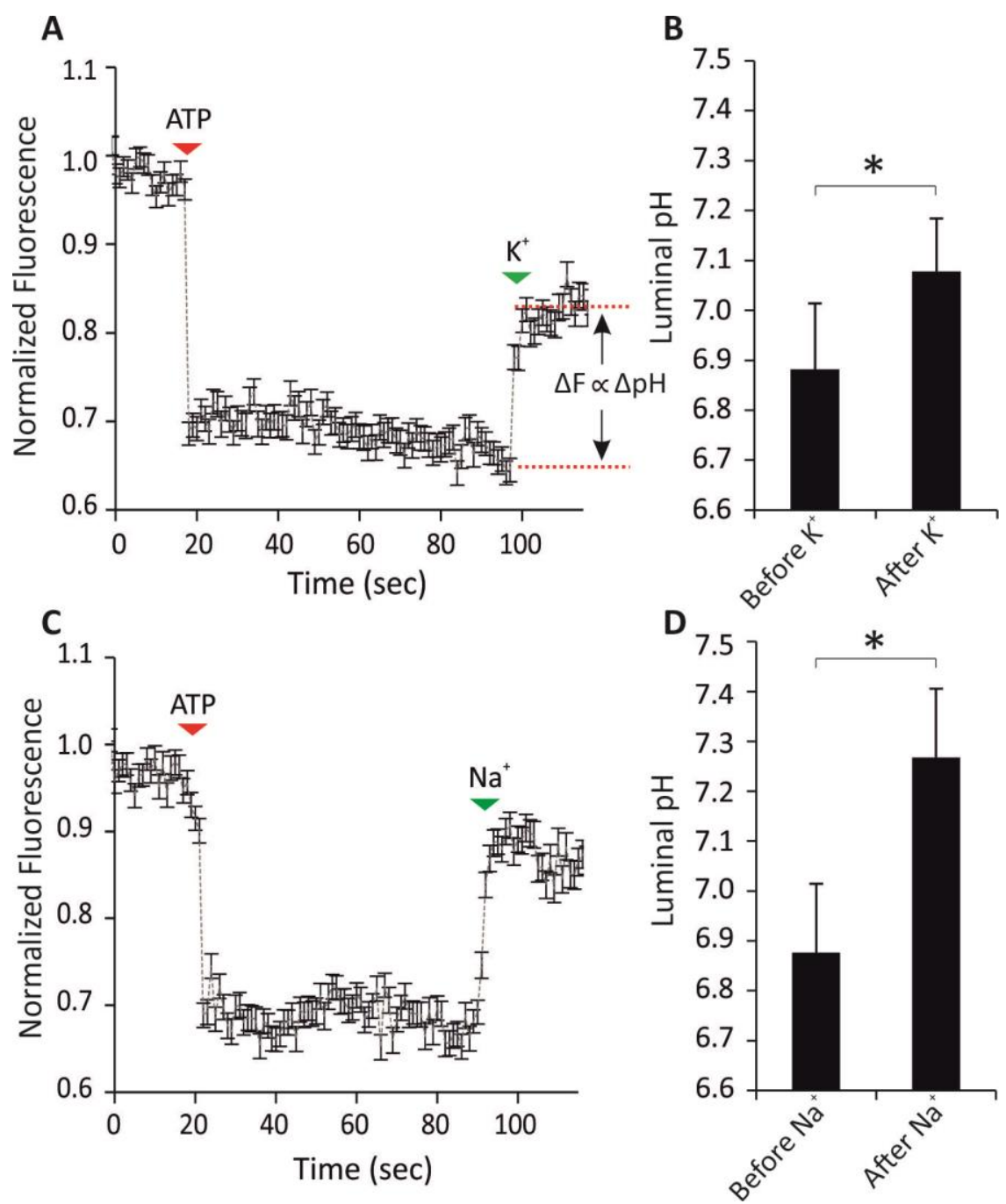

Figure 3-15 Effect of $\mathrm{K}^{+}$and $\mathrm{Na}^{+}$on the pH gradient across the membrane of SVs.

Representative fluorescence traces of spH-SVs in glycine buffer in response to addition of $1 \mathrm{mM}$ Mg-ATP and $30 \mathrm{mM} \mathrm{K-gluconate} \mathrm{(A)} \mathrm{or} 10 \mathrm{mM}$ Na-gluconate (C). Error bars represent SEM of $\mathrm{n}$ single SVs ( $\mathrm{n}=548$ and 633 in A and C, respectively). Addition of both $\mathrm{K}^{+}$(B) and $\mathrm{Na}^{+}$(D) significantly alkalinized the luminal $\mathrm{pH}$, proposing a cation $/ \mathrm{H}^{+}$antiport mechanism. Error bars represent SD of 4-6 experimental replicates. p-value $=1.1 \times 10^{-4}$ and $2.4 \times 10^{-3}$ in $\mathrm{B}$ and $\mathrm{D}$, respectively. 
In order to measure $\Delta \mathrm{pH}$ induced by $\mathrm{K}^{+}$or $\mathrm{Na}^{+}$, individual fluorescence traces were first converted to $\mathrm{pH}$ traces using the fluorescence-pH calibration curve (Figure 2-12). Then $\mathrm{Na}^{+} / \mathrm{K}^{+}$-induced $\Delta \mathrm{pH}$ was calculated as the difference between the averaged $\mathrm{pH}$ values before and after the chemical perturbations. Low concentrations of $\mathrm{Na}^{+}$resulted in a significantly alkalinized vesicular lumen $(\Delta \mathrm{pH}>0)$, whereas higher concentrations of $\mathrm{K}^{+}$were required to induce a comparable $\Delta \mathrm{pH}$ (Figure 3-16). This is in line with a previous study where a much lower $K_{\mathrm{m}}$ was reported for $\mathrm{Na}^{+}$compared to $\mathrm{K}^{+}$for crude SV alkalinization (Goh et al., 2011).
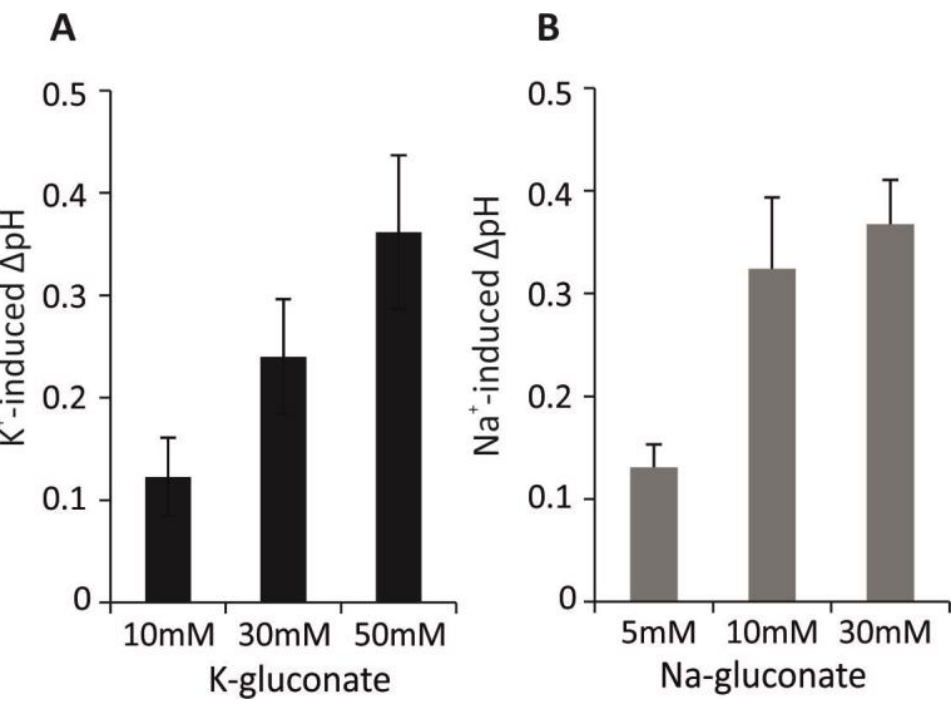

\section{Figure 3-16 Dose-dependent response of spH-SVs to $\mathrm{K}^{+}$and $\mathrm{Na}^{+}$.}

Fluorescence changes induced by addition of different concentrations of K-gluconate (A) and Nagluconate (B) to acidified SVs were converted to $\Delta \mathrm{pH}$ using the fluorescence-pH calibration curve. $\mathrm{K}^{+}$-induced alkalinization was larger upon addition of higher concentrations of $\mathrm{K}$ gluconate. This is while the effect of Na.gluconate on $\mathrm{pH}$ gradient reached saturation at much lower concentration. Error bars represent SD of 3-6 experimental replicates.

$\mathrm{Na}^{+}$and $\mathrm{K}^{+}$transport in secretory and endocytic organelles has been attributed to different isoforms of $\mathrm{Na}^{+} / \mathrm{H}^{+}$exchangers (NHEs) (Nakamura et al., 2005). However, a recent study has shown that VGLUT also contributes to the transport of $\mathrm{K}^{+}$in SVs through proton exchange (Preobraschenski et al., 2014). To uncover the molecules responsible for the transport of these cations, $\mathrm{Na}^{+}$and $\mathrm{K}^{+}$-induced alkalinization was measured in the presence of $50 \mu \mathrm{M}$ EIPA (5-N-ethyl-N-isopropyl amiloride), an inhibitor of most of NHEs (Goh et al., 2011), in the bath solution. Surprisingly, while the $\mathrm{Na}^{+}$effect was substantially decreased by EIPA, no significant effect was observed on $\mathrm{K}^{+}$-induced alkalinization (Figure 3-17). 


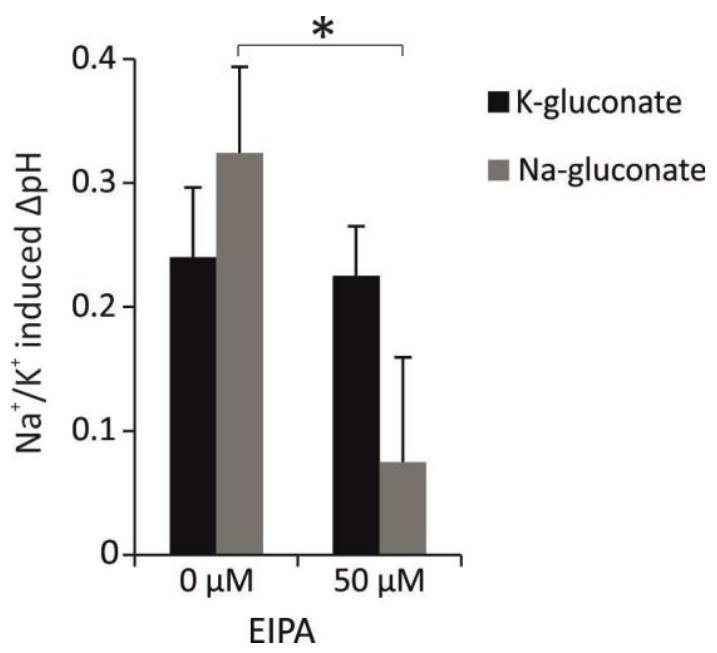

Figure 3-17 Inhibition of $\mathrm{Na}^{+} / \mathrm{K}^{+}$alkalinization by EIPA.

To unravel the responsible molecules for $\mathrm{K}^{+}$and $\mathrm{Na}^{+}$transport into the lumen of SVs, the effect of these cations on the $\mathrm{pH}$ gradient of acidified SVs was measured in the presence of EIPA, a general NHE blocker (no effect on the extent of acidification was observed in the presence of EIPA). While Na-induced alkalinization was significantly decreased in the presence of EIPA, no difference was observed in $\mathrm{K}^{+}$-induced alkalinization in the presence and absence of EIPA. Error bars represent SD of 3-6 experimental replicates.

These results agree well with a recent report that NHEs selectively transport $\mathrm{Na}^{+}$and not $\mathrm{K}^{+}$(Milosavljevic et al., 2014). Interestingly, when the $\Delta \mathrm{pH}$ induced by $10 \mathrm{mM}$ Nagluconate or $30 \mathrm{mM}$ K-gluconate was compared between glutamatergic and GABAergic SVs, a pronounced larger $\mathrm{K}^{+}$effect was observed in glutamatergic SVs. However, $\mathrm{Na}^{+}-$ induced alakalinization was equal for both SV populations (Figure 3-18). These results strongly suggest that an isoform of NHEs, probably NHE6 on SVs (Preobraschenski et al., 2014), mediates $\mathrm{Na}^{+}$influx into the vesicles. However, the greater relative effect of $\mathrm{K}^{+}$in glutamatergic compared to GABAergic vesicles as well as its resistance to EIPA lends further evidence to proposed $\mathrm{K}^{+} / \mathrm{H}^{+}$exchange by VGLUT (Preobraschenski et al., 2014). 


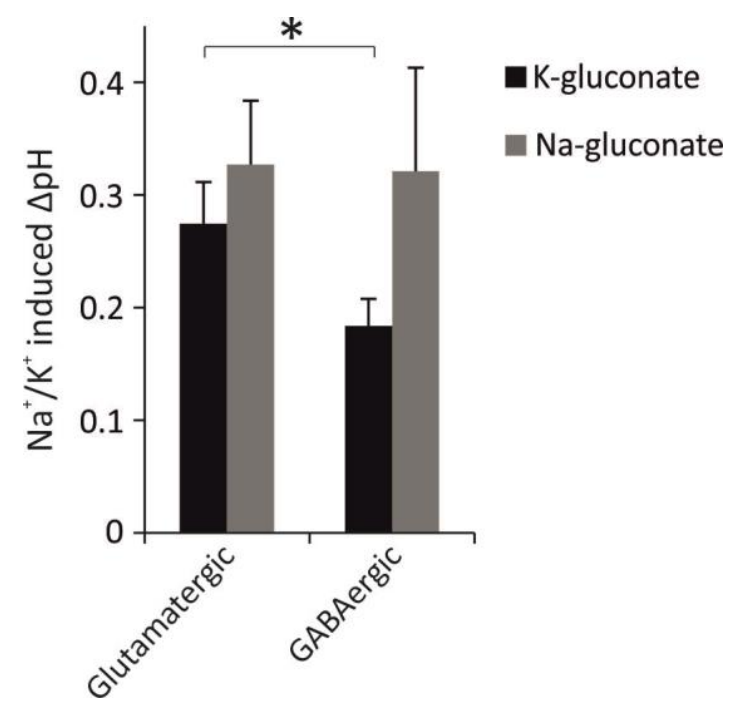

Figure 3-18 $\mathrm{Na}^{+} / \mathrm{K}^{+}$-induced alkalinization in glutamatergic and GABAergic SVs.

The extent of $\Delta \mathrm{pH}$ induced in the lumen of acidified SVs by addition of $30 \mathrm{mM}$ K-gluconate or 10 mM Na-gluconate was compared between glutamatergic and GABAergic SVs. While $\mathrm{K}^{+}$-induced alkalinization was significantly larger in glutamatergic SVs, no difference was observed between these vesicle subclasses in their response to $\mathrm{Na}^{+}$. Error bars represent SD of 3-5 experimental replicates. $\mathrm{p}$-value in the case of the $\mathrm{K}^{+}$-effect was 0.01 .

Next, the effect of $\mathrm{Na}^{+}$and $\mathrm{K}^{+}$on the membrane potential was measured. For this purpose, VF2.1.Cl-labeled SVs were acidified by $1 \mathrm{mM}$ Mg-ATP and then $10 \mathrm{mM} \mathrm{Na-}$ gluconate or $30 \mathrm{mM} \mathrm{K-gluconate}$ were applied to the bath solution. As shown in Figure 3-19, either no or only a slight increase in membrane potential was observed after $\mathrm{Na}^{+} / \mathrm{K}^{+}$addition. This further supports the cation $/ \mathrm{H}^{+}$exchange mechanism proposed by the pH measurements. 


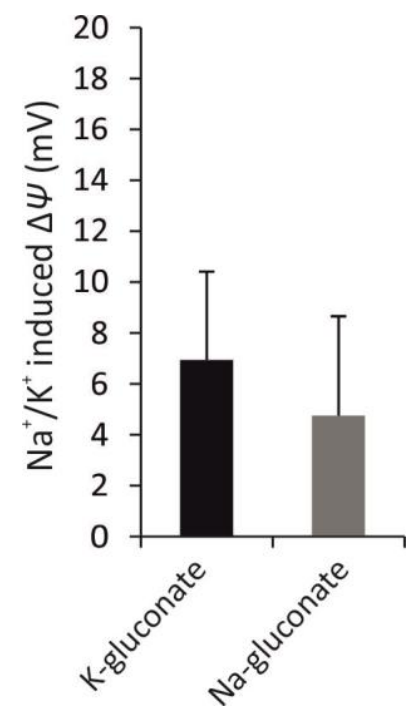

Figure 3-19 Effect of $\mathrm{Na}^{+}$and $\mathrm{K}^{+}$transport on the membrane potential across the membrane of SVs.

VF2.1.Cl-labeled SVs were acidified with $1 \mathrm{mM}$ Mg-ATP and then $10 \mathrm{mM}$ K-gluconate or $30 \mathrm{mM}$ Na-gluconate was added to the bath solution. Upon addition of these cations either no or only a slight increase was observed in the fluorescence of VF2.1.Cl, further suggesting a cation $/ \mathrm{H}^{+}$ exchange mechanism.

\subsubsection{Effect of Chloride on $\Delta \mu_{H+}$}

Acidification of spH-SVs by uncaging of $4 \mathrm{mM}$ NPE-ATP in the presence of $30 \mathrm{mM}$ TEA-Cl in glycine buffer led to significantly greater quenching of spH fluorescence, indicative of a lower luminal pH (Figure 3-20). Greater acidification with $\mathrm{Cl}^{-}$has been also reported by other studies in which acridine orange was used as a pH-sensitive probe (e.g. (Hnasko et al., 2010; Schenck et al., 2009)). 


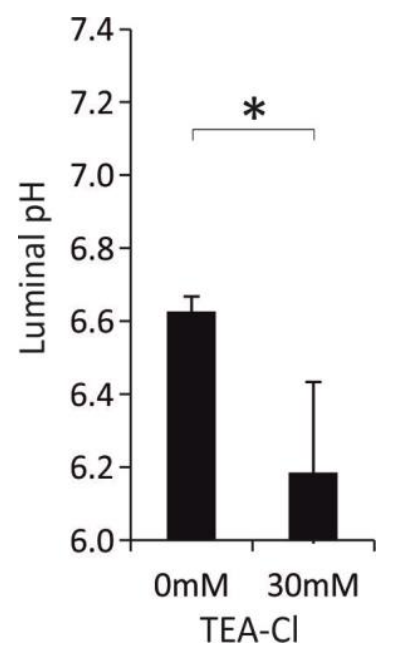

Figure 3-20 Effect of chloride on the pH gradient across the membrane.

The effect of $\mathrm{Cl}^{-}$on the averaged luminal $\mathrm{pH}$ of spH-SVs after acidification. Error bars represent SD of 3-5 experimental replicates. $\mathrm{p}$-value $=0.01$.

Next, the effect of $\mathrm{Cl}^{-}$on the $\mathrm{pH}$ gradient was compared in glutamatergic and GABAergic SVs. For this purpose, the averaged luminal $\mathrm{pH}$ achieved in the absence of $\mathrm{Cl}^{-}$ in each of these vesicle populations was subtracted from the luminal $\mathrm{pH}$ that the respective SVs reached with the same concentration of NPE-ATP but in the presence of $30 \mathrm{mM}$ TEA-Cl. Interestingly, the extent of $\mathrm{Cl}$-induced acidification $(\Delta \mathrm{pH}<0)$ was significantly greater in glutamatergic SVs compared to GABAergic SVs (Figure 3-21A). In addition, the glutamatergic vesicles acidified significantly faster than GABAergic SVs in the presence of $\mathrm{Cl}^{-}$(Figure 3-21B). 


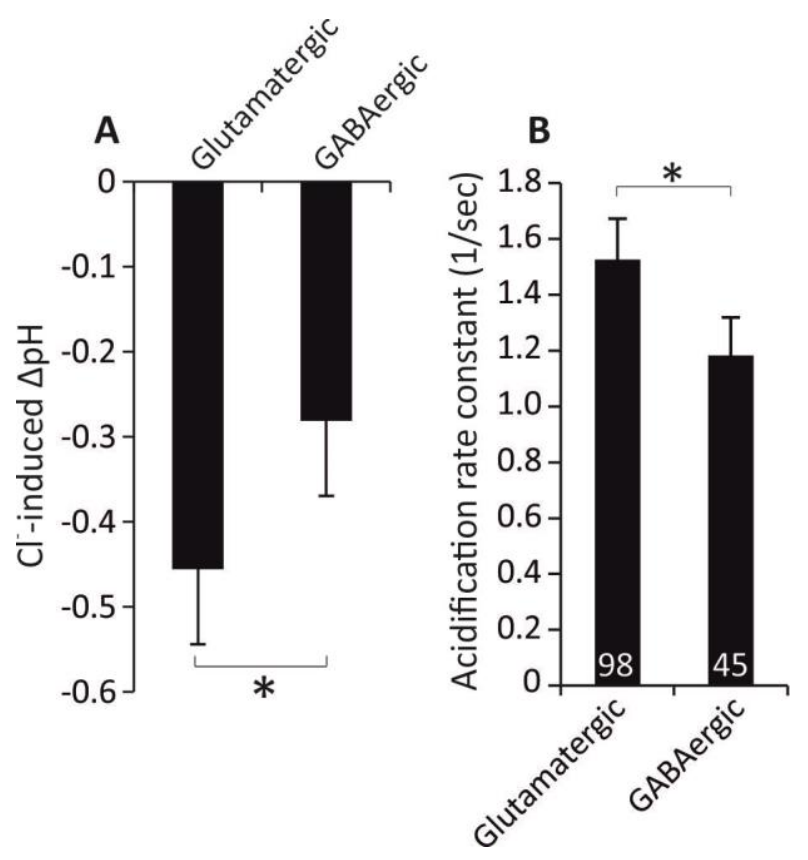

Figure 3-21 Chloride-induced acidification in glutamatergic and GABAergic SVs.

A) The extent of further acidification $(\Delta \mathrm{pH}<0)$ induced by including $30 \mathrm{mM}$ TEA-Cl in the bath solution was significantly greater in glutamatergic compared to GABAergic SVs. Error bars represent SD of 3 experimental replicates. p-value $=0.01$. B) The acidification of glutamatergic SVs was significantly faster than GABAergic SVs in the presence of $\mathrm{Cl}^{-}$in the bath solution. Averaged acidification rate constants $( \pm \mathrm{SEM})$ were $1.52 \pm 0.15(1 / \mathrm{sec})$ and $1.18 \pm 0.14(1 / \mathrm{sec})$ for the glutamatergic and GABAergic SVs, respectively. Error bars represent SEM of $\mathrm{n}$ single SVs ( $\mathrm{n}$ is indicated on bars). $\mathrm{p}$-value $=0.045$.

Furthermore, by addition of $\mathrm{Cl}^{-}$to acidified VF2.1.Cl-labeled SVs, a drop in membrane potential was observed, which was significantly larger in glutamatergic compared to GABAergic SVs (Figure 3-22). This is consistent with a larger $\mathrm{Cl}^{-}$effect on the $\mathrm{pH}$ gradient in glutamatergic SVs. 


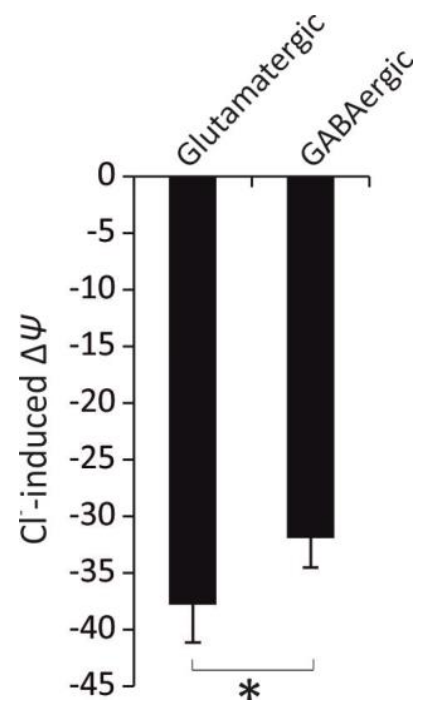

Figure 3-22 Effect of chloride on the membrane potential of SVs.

Addition of $20 \mathrm{mM}$ TEA-Cl to acidified SVs induced a drop in the membrane potential $(\Delta \psi<0)$ which was significantly greater in glutamatergic compared to GABAergic SVs. Together with a higher $\mathrm{Cl}^{-}$-induced acidification in glutamatergic SVs, these data suggest larger $\mathrm{Cl}^{-}$influx in glutamatergic SVs. Error bars represent SD of 3 experimental replicates. p-value $=0.006$.

\subsection{Effect of Neurotransmitters on Electrochemical Gradient}

The electrochemical gradient across the membrane provides the free energy required for accumulating neurotransmitters against a concentration gradient. While it is wellaccepted that $\Delta \mu_{\mathrm{H}+}$ can regulate uptake of different neurotransmitters, it is poorly understood how transport of neurotransmitters can affect $\Delta \mu_{\mathrm{H}+}$. To this end, the effect of glutamate and GABA on the two components of the electrochemical gradient were measured.

\subsubsection{Effect of Glutamate on $\Delta \mu_{H+}$}

To measure the effect of glutamate on the $\mathrm{pH}$ gradient across the membrane, spHSVs were acidified in the presence of $10 \mathrm{mM}$ glutamate in the bath solution. As shown in Figure 3-23A, the lumen of SVs reached a significantly lower $\mathrm{pH}$ in the presence of glutamate. However, the acidification kinetics of the SVs remained unchanged (Figure 3-23B). Due to the presence of other VGLUT isoforms on GABAergic SVs (Edwards, 2007; Zander et al., 2010), a glutamate-induced acidification was also observed in these vesicles. However, the extent of acidification $(\Delta \mathrm{pH}<0)$ was significantly larger in glutamatergic vesicles (Figure 3-23C). 

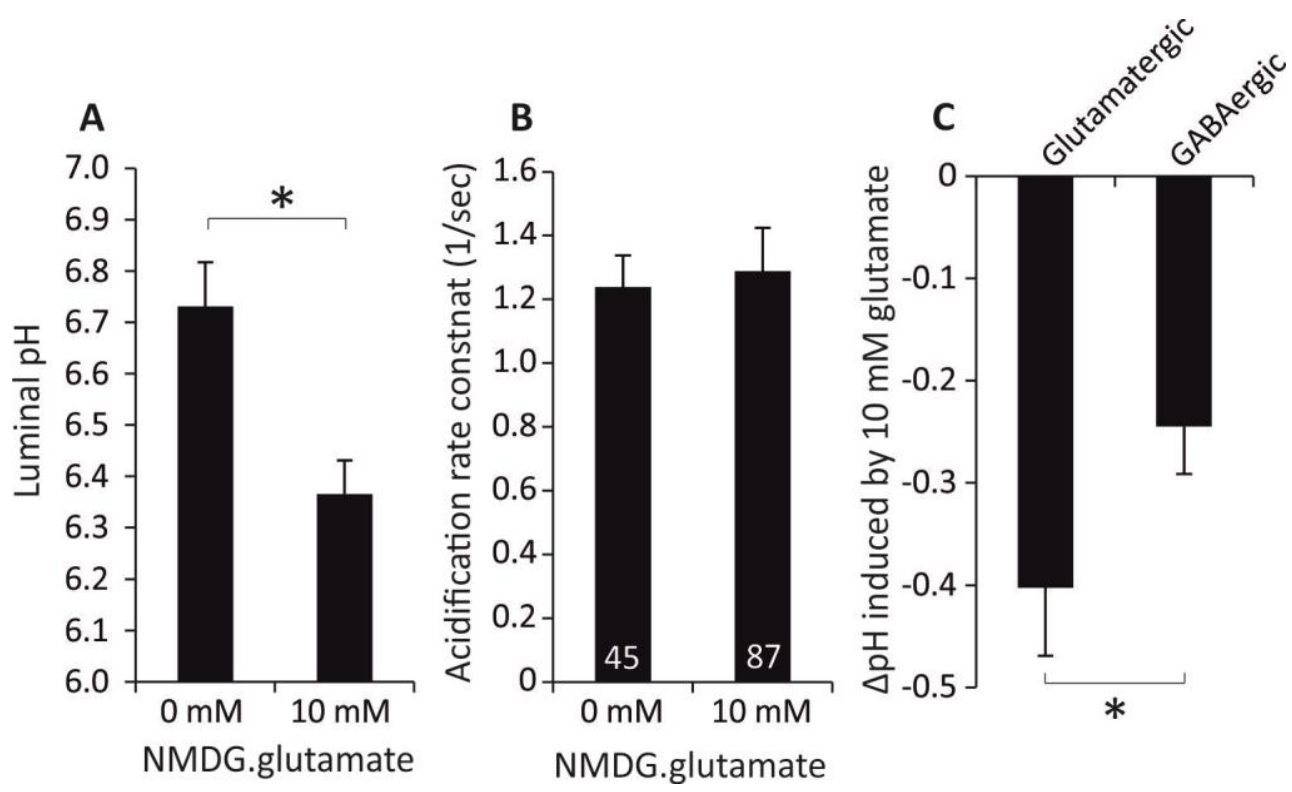

Figure 3-23 Effect of glutamate on the pH gradient of SVs.

A) Averaged luminal $\mathrm{pH}$ of spH-SVs after uncaging of $2 \mathrm{mM}$ NPE-ATP in the presence and absence of $10 \mathrm{mM}$ NMDG-glutamate in the bath solution. Error bars represent SD of 3-5 experimental replicates. $\mathrm{p}$-value $=0.005$. B) Acidification rate constant of spH-SVs was not affected by the presence of NMDG-glutamate in the bath solution (also no significant difference was observed when acidification rate constants of glutamatergic and GABAergic SVs were compared separately (data not shown)). Averaged acidification rate constants ( \pm SEM) were $1.23 \pm 0.10(1 / \mathrm{sec})$ and $1.28 \pm 0.13(1 / \mathrm{sec})$ in the absence and presence of $10 \mathrm{mM}$ glutamate, respectively. Error bars indicate SEM ( $\mathrm{n}$ is indicated on the bars). C) Glutamate-induced acidification was also observed in GABAergic SVs albeit was significantly less than for glutamatergic SVs. Error bars represent SD of 3 experimental replicates. $p$-value $=0.028$.

Glutamate-induced acidification has been attributed to its negative charge, which decreases $\Delta \psi$ upon accumulation and would allow the V-ATPase to pump more protons (Budzinski et al., 2011). Indeed, addition of glutamate to acidified SVs led to a decrease in VF2.1.Cl fluorescence, indicating a drop in membrane potential (Figure 3-24A). Moreover, consistent with a VGLUT mediated $\mathrm{K}^{+}$flux, dissipation of the membrane potential by glutamate uptake was significantly mitigated by the presence $\mathrm{K}^{+}$ (Figure 3-24B). 

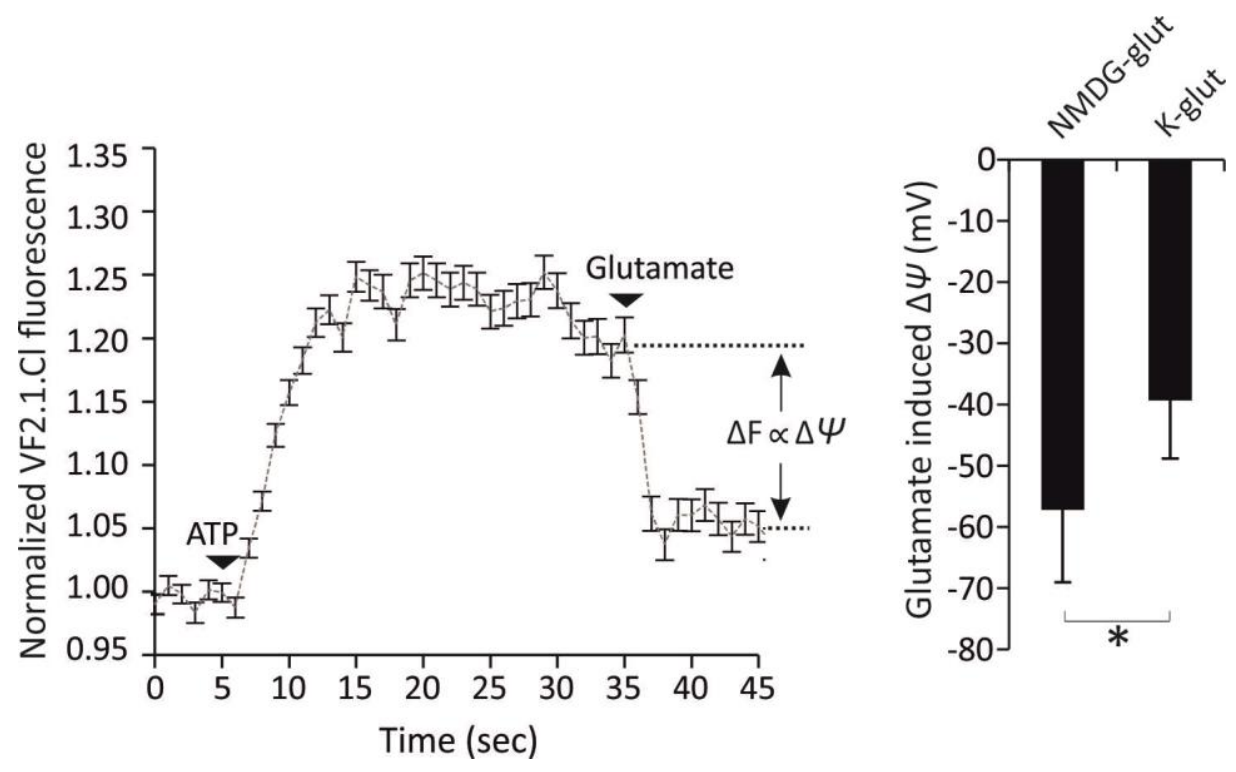

Figure 3-24 Effect of glutamate on membrane potential across the membrane.

A) Representative fluorescence trace of VF2.1.Cl-labled SVs in response to $3 \mathrm{mM} \mathrm{Mg-ATP}$ and 10 mM NMDG-glutamate. As shown, uptake of glutamate led to a drop in membrane potential. Error bars represent SEM of $\mathrm{n}$ single SVs (n = 952). B) Glutamate induced $\Delta \psi$ was significantly lower when it was added as a $\mathrm{K}^{+}$salt instead of $\mathrm{NMDG}^{+}$salt. In line with more pronounced $\mathrm{K}$-induced alkalinization in glutamatergic SVs, this data suggest $\mathrm{K}^{+}$influx by VGLUT. Error bars indicate SD of 3-5 experimental replicates. p-value $=0.02$.

As an alternative explanation for the observed glutamate induced acidification, it has recently been proposed that glutamate-induced acidification may also be due to the buffering effect of glutamate (Hnasko et al., 2010). To test this hypothesis, spH-SVs were acidified in the presence of $10 \mathrm{mM}$ NMDG-glutamate and the buffering capacity was measured as described in section 2.7.4. In the presence of glutamate, the interior of SVs acidified to $\mathrm{pH} \sim 6.3$. However, in a completely anion-free environment, the SVs did not reach a pH below 6.5. Therefore, to estimate the endogenous buffering capacity at $\mathrm{pH} \sim$ 6.3 , the previously-determined buffering capacity and $\mathrm{pH}$ relationship was extrapolated to this $\mathrm{pH}$ (Figure 3-25). The mean value of the buffering capacity at $\mathrm{pH} \sim 6.3$ was then subtracted from the measured buffering capacity in the presence of glutamate. This resulted in $\sim 20 \mathrm{mM} / \Delta \mathrm{pH}$ extra buffering capacity induced by glutamate. 


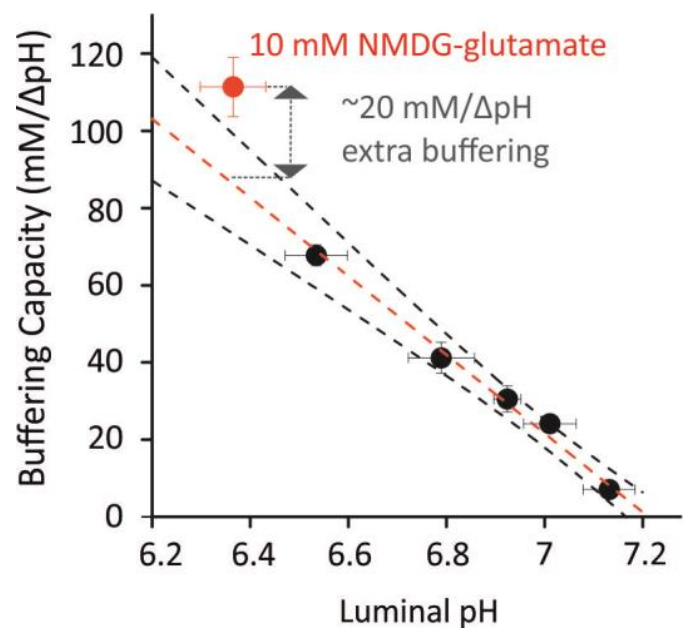

\section{Figure 3-25 Buffering effect of glutamate.}

Buffering capacity of SVs was measured in the absence (black circles) and presence of $10 \mathrm{mM}$ NMDG-glutamate (red circle). Red line indicates the linear fit to data in the absence of glutamate, and black lines show the upper and lower bounds of $95 \%$ confidence interval of the fit. Subtracting the mean value of buffering capacity at $\mathrm{pH} \sim 6.3$ from the measured buffering capacity in the presence of glutamate (gray line and arrow) resulted in $\sim 20 \mathrm{mM} / \Delta \mathrm{pH}$ extra buffering effect. Both the horizontal and vertical error bars represent SD of 3-5 experimental replicates.

\subsubsection{Effect of GABA on $\Delta \mu_{H+}$}

To assess the effect of GABA on $\Delta \mu_{\mathrm{H}+}$, measurements similar to those performed with glutamate were performed with $10 \mathrm{mM} \mathrm{GABA}$ in K-gluconate buffer as the bath solution. GABAergic SVs reached a significantly lower luminal $\mathrm{pH}$ in the presence of GABA compared to without GABA (Figure 3-26A), while no change was observed in their acidification kinetics (Figure 3-26B). It should be mentioned that the effect of GABA on $\mathrm{pH}$ gradient was also observed in glutamatergic SVs, which was significantly less than the effect of GABA in GABAergic SVs. 


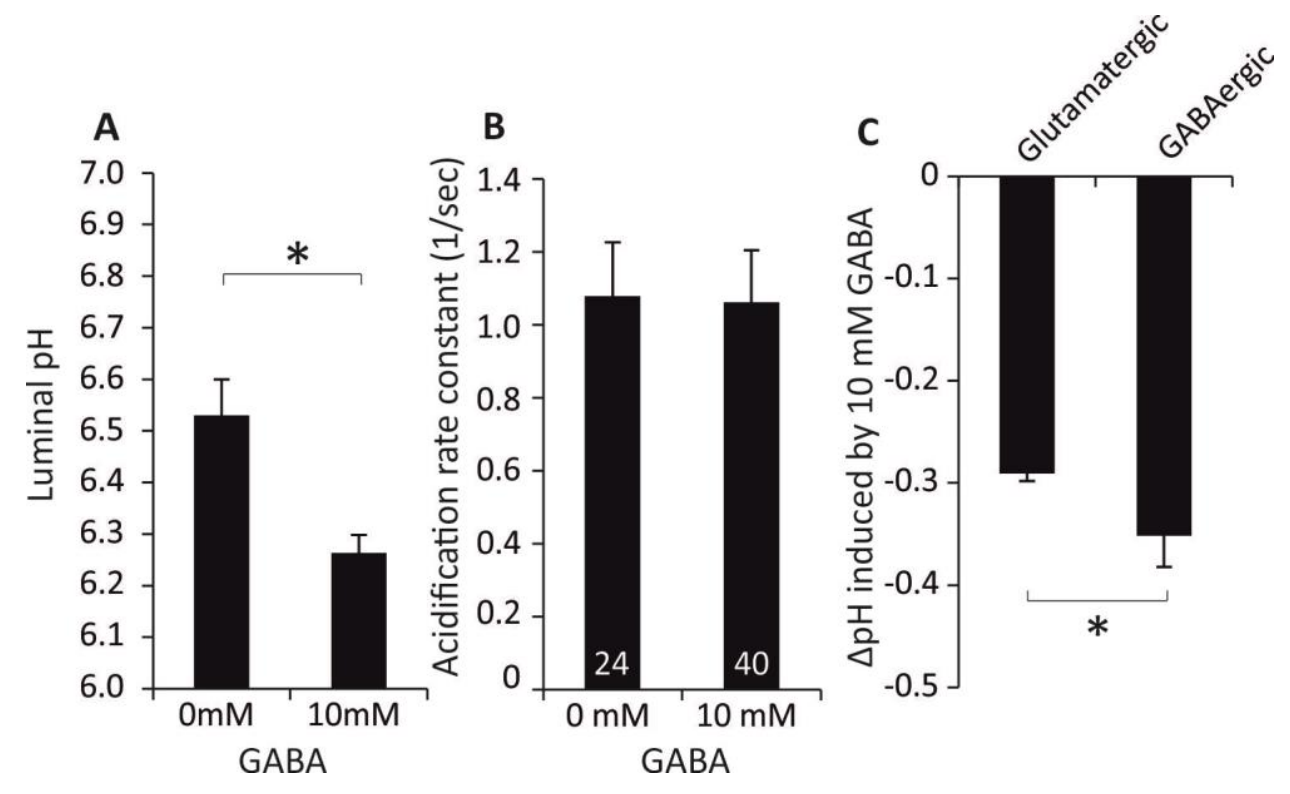

Figure 3-26 Effect of GABA on the pH gradient of SVs.

A) Averaged luminal $\mathrm{pH}$ of GABAergic SVs after uncaging of $4 \mathrm{mM}$ NPE-ATP, in the absence and presence of $10 \mathrm{mM}$ GABA in K-gluconate buffer as the bath solution. Significantly lower luminal pH was measured in the presence of GABA. Error bars indicate SD of 3-5 experimental replicates. p-value $=0.004$. B) No significant difference was observed in the acidification rate constant of GABAergic SVs in the presence and absence of GABA. Averaged acidification rate constants $( \pm$ SEM) were $1.08 \pm 0.15(1 / \mathrm{sec})$ and $1.06 \pm 0.14(1 / \mathrm{sec})$ in the absence and presence of $10 \mathrm{mM}$ GABA, respectively. Error bars represent SEM (n is indicated on the bars). C) GABA-induced acidification was also observed in glutamatergic SVs which was significantly less compared to GABAergic SVs. Error bars represent SD of 3 experimental replicates. p-value $=0.014$.

Moreover, addition of GABA to acidified GABAergic SVs led to a drop in membrane potential (Figure 3-27A). Since GABA has no net charge, this further implies that GABA transport is coupled to proton efflux from the vesicular lumen. This view has been challenged recently by a proposed GABA/ $\mathrm{Cl}^{-}$co-transport mechanism (Juge et al., 2009). To test this hypothesis, the GABA-induced $\Delta \psi$ was measured in the presence of $5 \mathrm{mM}$ TEA-Cl, the $\mathrm{Cl}^{-}$concentration at which GABA uptake was reported to be maximal (see Fig1E of (Juge et al., 2009)). In case a $\mathrm{GABA} / \mathrm{Cl}^{-}$co-transport mechanism were to exist, the GABA-induced $\Delta \psi$ should be significantly larger in the presence of $\mathrm{Cl}^{-}$. However, no significant difference was observed in the presence of $\mathrm{Cl}^{-}$in the bath solution compared to without $\mathrm{Cl}^{-}$(Figure 3-27B). 

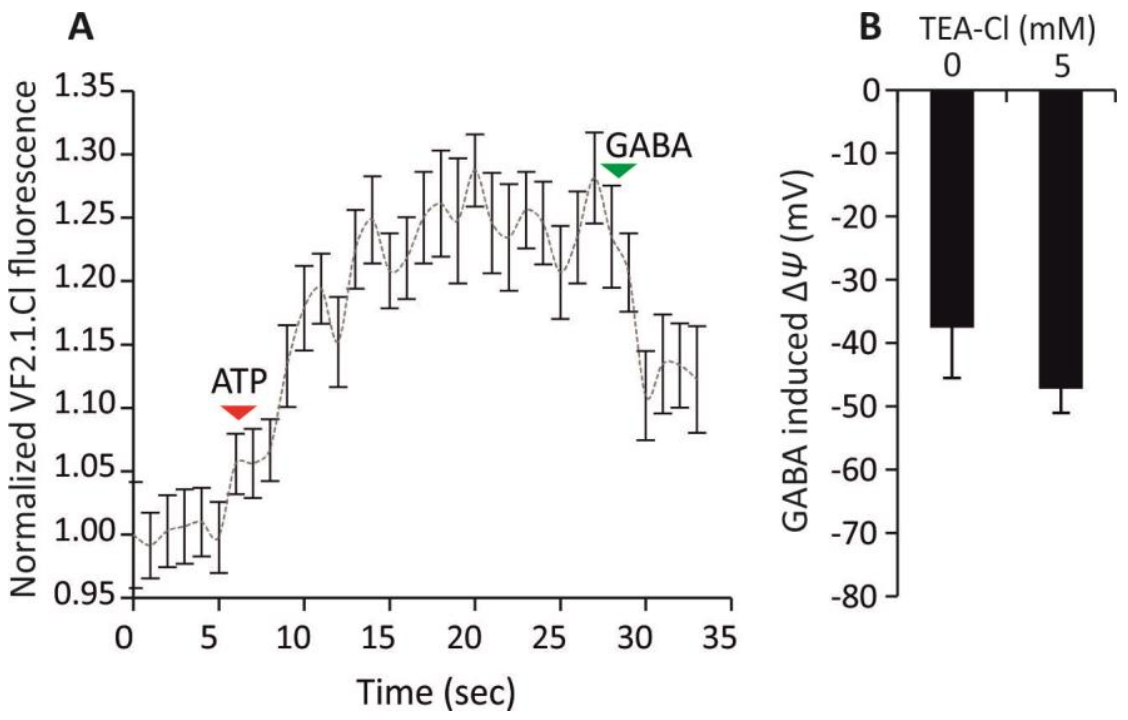

Figure 3-27 Effect of GABA on the membrane potential across the membrane of SVs.

A) Representative fluorescence trace of VF2.1.Cl labeled GABAergic SVs in response to $3 \mathrm{mM} \mathrm{Mg}$ ATP and $10 \mathrm{mM}$ GABA. Error bars represent SEM of $\mathrm{n}$ single SVs, $\mathrm{n}=50$. B) No significant difference was observed between GABA-induced $\Delta \psi$ in the absence and presence of $5 \mathrm{mM}$ TEA.Cl in the bath solution. Error bars indicate SD of 3-5 experimental replicates. 


\section{Discussion}

In this thesis, a combination of advanced biochemical and biophysical approaches has been employed to study the regulation of $\Delta \mu_{\mathrm{H}+}$ in SVs in detail and unravel how this gradient can efficiently provide the driving force for distinct vesicular transporters with different bioenergetics characteristics. For this purpose, as presented in the 'Results' section, the responses of the two components of $\Delta \mu_{\mathrm{H}+}$ to various chemical and electrical perturbations were measured at the single vesicle level. These data provided valuable information about less studied biophysical characteristics of SVs such as their buffering capacity and proton permeability. Furthermore, by side-by-side comparison of glutamatergic and GABAergic vesicles at the singly vesicle level, subtle and yet unresolved differences between these vesicle populations could be detected.

\subsection{The Relationship between $\Delta p H$ and $\Delta \psi$ Is not Linear}

As shown in Figure 3-4 and Figure 3-5, acidification of SVs at different ATP concentrations produced a decrease in luminal $\mathrm{pH}$ and a build-up of membrane potential in a dose-dependent manner. Plotting $\Delta \mathrm{pH}$ against $\Delta \psi$ for different ATP concentrations revealed that there is a non-linearity between these two components of $\Delta \mu_{\mathrm{H}+}$. The relationship between $\Delta \mathrm{pH}$ and $\Delta \psi$ can be described by Eq. 1.5. However, since the acidification and potentiometric measurements were performed in the absence of membrane-permeable cations and anions (glycine buffer, Table 2-1), Eq. 4.1 can be derived from Eq. 1.5:

$$
\Delta \psi=\frac{F V}{C} \times \beta \times \Delta \mathrm{pH}
$$

where $F$ is Faraday's constant, $V$ is the volume of the organelle, $C$ is the total capacitance of the membrane and $\beta$ is the buffering capacity. According to this equation, the relationship between $\Delta \mathrm{pH}$ and $\Delta \psi$ is shaped by the luminal buffering capacity. Thus, the observed non-linearity between these two parameters implies that the buffering capacity of SVs is not constant. Indeed, upon measuring buffering capacity at different luminal $\mathrm{pH}$ values, it was observed that $\beta$ increases as the lumen of SVs acidified. This is in line with the reported variable $\beta$ in other organelles such as endocytic vesicles (Van 
Dyke and Belcher, 1994) and lysosomes (Van Dyke, 1993). Moreover, it has been shown that cytosolic buffering capacities in many cells such as leukocytes (Goldsmith and Hilton, 1992), lymphocytes (Saleh and Batlle, 1990), cardiac cells (Vaughan-Jones and $\mathrm{Wu}, 1990$ ) and snail neurons (Szatkowski and Thomas, 1989) changes at different $\mathrm{pH}$.

This dynamic buffering capacity can significantly facilitate fast vesicle filling with neurotransmitters. The lack of strong buffering capacities at high luminal $\mathrm{pH}$ allows for a swift $\mathrm{pH}$ drop upon proton influx and thus for fast formation of $\Delta \mu_{\mathrm{H}+}$. In addition, the high buffering capacity in the acidified lumen implies that the majority of luminal buffers have a $\mathrm{p} K_{\mathrm{a}}<6.5$, which would prevent excessive acidification of the SV lumen by the VATPase.

So far, the magnitude of luminal buffering capacity of SVs has been reported by only two studies, both of which used a similar approach as employed in this thesis to measure $\beta$ at different luminal pH (Budzinski et al., 2011; Egashira et al., 2015). However, the experimental system was different in these two studies. Budzinski, et al. (2011) used single spH-SVs and reported the averaged luminal $\beta$ of SVs to be $139 \pm 29$ $\mathrm{mM} / \Delta \mathrm{pH}$, while in work by Egashira, et al. (2015), cultured hippocampal neurons expressing mOrange2 were used and an averaged $\beta$ of $57.4 \pm 4.8 \mathrm{mM} / \Delta \mathrm{pH}$ was measured. In the current study, an averaged $\beta$ between pH 7 and 6.5 was measured to be $\sim 35 \mathrm{mM} / \Delta \mathrm{pH}$, and by extrapolating the $\beta$ and $\mathrm{pH}$ relationship to $\mathrm{pH} 5.7$, averaged $\beta$ is estimated to be $\sim 53 \mathrm{mM} / \Delta \mathrm{pH}$, close to the results of Egashira, et al. (2015).

The absolute value of $\beta$ can fluctuate based on the experimental conditions and used buffers/solutions. Thus, it has to be interpreted cautiously. However, the $\beta$ values obtained from these studies fall in the range of reported $\beta$ for other intracellular compartments $(20-100 \mathrm{mM} / \Delta \mathrm{pH})$ such as the ER, Golgi, endosomes and lysosomes (Gekle and Silbernagl, 1995; Grabe and Oster, 2001; Van Dyke, 1993; Van Dyke and Belcher, 1994).

\subsection{Synaptic Vesicles Acidify in less than One Second}

The cytosolic concentration of ATP at the presynaptic terminal is estimated to be $\sim 2$ mM (Rangaraju et al., 2014). At this ATP concentration, the time constant of acidification ( $\tau_{\text {acidification}}$ ) of individual SVs was estimated to be $\sim 800 \mathrm{~ms}$ (Figure 3-4C). This value is

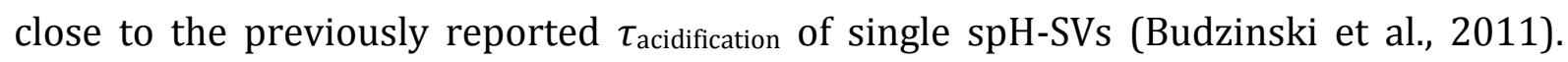
However, it is much faster than reported values by other studies where the same probe 
(pHluorin) was used to measure the acidification kinetics of SVs in cultured hippocampal neurons (Atluri and Ryan, 2006; Gandhi and Stevens, 2003; Granseth et al., 2006; Kwon and Chapman, 2011). In contrast to the single vesicle imaging in this thesis as well as the work of Budzinski, et al. 2011, the averaged acidification kinetics of a bulk of early endocytosed SVs is measured in cultured neurons. This may lead to a potential underestimation of the acidification speed. Moreover, it is not known whether earlyendocytosed SVs, which are still coated with clathrin, are able to acidify or whether uncoating is a prerequisite (Milosevic et al., 2011). The latter case would impose a delay between endocytosis and acidification that would explain the difference between the two experimental systems. In addition, it has been shown that increasing the temperature from $25^{\circ} \mathrm{C}$ to $35^{\circ} \mathrm{C}$ does significantly accelerate both endocytosis and the acidification rate of SVs at hippocampal nerve terminal (Granseth and Lagnado, 2008), suggesting that the kinetics of membrane retrieval are affecting the measured acidification kinetics at cultured neurons.

Fast acidification of SVs is vital for rapid SV recycling. It has been proposed in many studies that recycling vesicles constitute only a minor fraction of the total vesicle population at the presynaptic terminal (de Lange et al., 2003; Denker et al., 2011; Harata et al., 2001; Kavalali, 2006; Marra et al., 2012; Richards et al., 2003). This implies that during repetitive synaptic activity, a single SV has only limited time to accomplish endocytosis, neurotransmitter filling including SV acidification, and exocytosis. It has been estimated that a single SV at hippocampal synapses is reused within 1 second (Sara et al., 2002) to a few seconds (Pyle et al., 2000) during different stimulation frequencies, demonstrating that the acidification of SV lumen must occur with sub-second kinetics. However, the rate of SV reuse at different synapses varies and depends on the synaptic activity (Denker et al., 2011). For instance, it has been shown that at the calyx of Held, during stimulation at 20-50 Hz, SVs are reused within 30-80 seconds (Qiu et al., 2015). Nonetheless, considering the slow kinetics of endocytosis (15-20 s) (Dittman and Ryan, 2009; Korber et al., 2012) and neurotransmitter uptake ( 15 s) (Hori and Takahashi, 2012), this would not contradict the demand for fast acidification at these synapses. 


\subsection{Glutamatergic SVs Generate Larger Electrochemical Gradient Compared to GABAergic SVs}

Acidification and potentiometric measurements revealed that in response to a constant concentration of ATP in the absence of membrane-permeable ions, glutamatergic SVs generate $11.99 \pm 5.2 \mathrm{mV}$ larger $\Delta \psi$ and $0.1 \pm 0.03$ larger $\Delta \mathrm{pH}$ across their membrane compared to GABAergic SVs. According to Eq. 1.4, these differences result in an overall $17.79 \pm 6.9 \mathrm{mV}$ greater $\Delta \mu_{\mathrm{H}+}$ in glutamatergic SVs. There are three attributes, which can strongly influence the magnitude of $\Delta \mu_{\mathrm{H}+}$, and thus may contribute to the variation observed between glutamatergic and GABAergic SVs: (i) the rate of proton influx, (ii) the buffering capacity and (iii) the rate of proton efflux (Grabe and Oster, 2001). To unravel the underlying mechanism, these factors were measured in glutamatergic and GABAergic SVs.

If the greater $\Delta \mu_{\mathrm{H}_{+}}$in glutamatergic SVs were due to their rate of proton influx, these SVs should have a significantly faster acidification rate, which in turn would require them to possess a greater copy-number of the V-ATPase. However, as shown in Figure 3-7C, no significant difference was observed between acidification rate constants of these vesicles compared to GABAergic SVs. This result is in line with the past proteomic analysis in which no significant differences were observed in expression of different subunits of the V-ATPase in these two vesicle subclasses (Gronborg et al., 2010).

Next, the buffering capacity, a second attribute which influences the amount of free protons in the lumen, was compared between these SV populations. It should be noted that a variation in buffering capacity can only explain the observed difference in the magnitude of $\Delta \mathrm{pH}$, and in this case, an additional charge regulating mechanism would be required to cause the observed difference in $\Delta \psi$. However, no significant difference was observed in the luminal buffering capacity between the glutamatergic and GABAergic SVs. Since buffering capacity can influence the dynamics of acidification by proton influx into vesicles (Grabe and Oster, 2001), these results are consistent with the similar acidification rates in these SVs.

With still no explanation for the observed difference, the proton efflux rates were compared between glutamatergic and GABAergic SVs. Interestingly, a significantly faster efflux rate was measured for GABAergic SVs (Figure 3-11). This result can reasonably explain the observed difference in the magnitude of $\Delta \mu_{\mathrm{H}+}$ between glutamatergic and 
GABAergic SVs: a higher proton efflux will result in a lower luminal proton contents in GABAergic SVs, which in turn would affect both components of $\Delta \mu_{\mathrm{H}+}$ and lead to an overall smaller $\Delta \mu_{\mathrm{H}+}$. By incorporating the measured efflux rates into Eq. 2.4 and 2.5, the proton permeabilities of GABAergic and glutamatergic SVs were estimated to be $15.2 \mathrm{x}$ $10^{-3}$ and $13.5 \times 10^{-3} \mathrm{~cm} / \mathrm{s}$, respectively. These values are close to the previously reported proton permeability of SVs $\left(2.1 \times 10^{-2} \mathrm{~cm} / \mathrm{s}\right)$ (Budzinski et al., 2011), and also to the values reported for other intracellular compartments $\left(0.75-4.8 \times 10^{-3} \mathrm{~cm} / \mathrm{s}\right.$ ) (Chandy et al., 2001; Grabe and Oster, 2001; Van Dyke, 1993).

Since glutamatergic and GABAergic SVs were shown to have a similar size, and assuming that these vesicles do not differ in their lipid composition, the greater proton permeability of GABAergic SVs cannot be attributed to a higher passive leakage through the membrane. Therefore, it was proposed that the greater proton efflux is mediated by their vesicular transporters (VGAT) (Figure 3-12 and Figure 3-13). This would imply that VGAT is permeable to protons. As will be discussed in the following section, other experiments indeed supported the proton permeability of VGAT and showed that VGAT functions as a proton exchanger, which couples the proton efflux to the transport of GABA molecules.

However, as discussed in section 1.2.2.3, there is considerable evidence that VGLUT is also permeable to protons and its activity involves proton exchange (e.g. (Bai et al., 2001; Bellocchio, 2000; Preobraschenski et al., 2014; Takamori et al., 2002)). The observed higher permeability in GABAergic SVs would imply that VGAT is more proton permeable compared to VGLUT. This in turn would suggest that these transporters might have a distinct transport mechanism. While there is a scarcity of details regarding the mechanistic principles of these transporters, a number of structures of their bacterial/archaeal homolog have been elucidated in various states by crystallography (Yamashita et al., 2005; Yernool et al., 2004). Although the overall sequence identity between vesicular transporters and the prokaryotic counterparts is rather low, there are highly conserved residues in these transporters including functionally important residues which can also be found in other members of SLC superfamily (see (Yamashita et al., 2005; Yernool et al., 2004) for amino acid sequence alignments of LeuT and GltPh with different members of SLCs, respectively). Perhaps comparing the transport mechanism of LeuT, a eubacterial orthologue of the GABA and other biogenic amine transporters, with GltPh, an archebacterial orthologue of the glutamate/aspartate and 
neutral amino acid transporters (Gouaux, 2009), can provide us with some helpful clues to understand the observed difference between VGAT and VGLUT.

LeuT and GltPh, both utilize the $\mathrm{Na}^{+}$electrochemical gradient to shuttle their substrates with a co-transport mechanism, which is leucine for LeuT and aspartate for $\mathrm{GltPh}$. The transport in both transporters involves a conformational switch, which as mentioned in the 'Introduction' section, is referred to as 'alternating access'. However, there are two versions of the alternating access: the 'rocker switch' and the 'two-gated pore' (Focke et al., 2013). In the rocker switch, a rigid-body rocking motion of two domains of the transporter, relative to each other, is required to expose the substrate/ion binding pocket to either sides of the membrane in an alternating fashion, while in the two-gated pore, a transmembrane pore is terminated with two gates at each side of the transporter and the substrate/ion binding site which locates between the pores, is available from the side where the gate is open (Figure 4-1) (Focke et al., 2013).

A

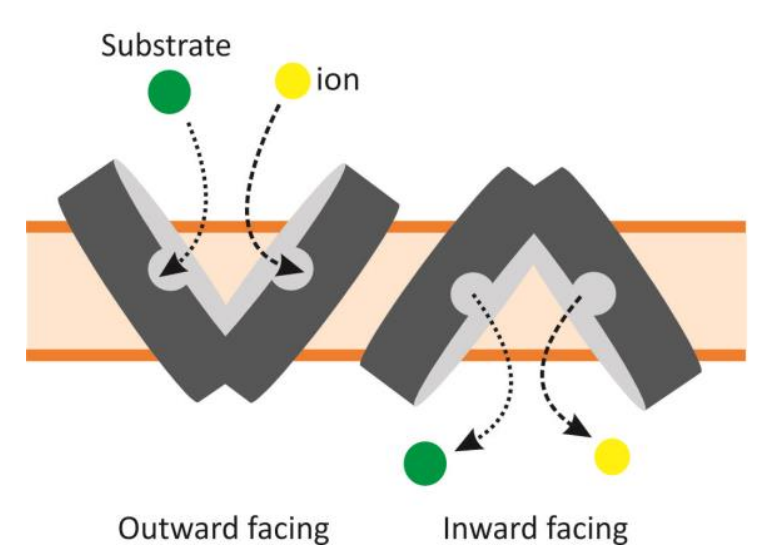

B

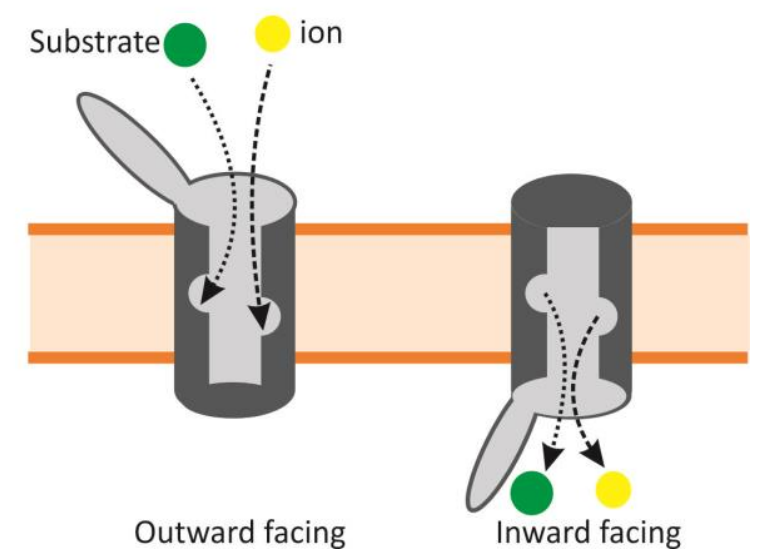

Figure 4-1 Two versions of alternating access for membrane transporters.

A) In the rocker switch model, the ion/substrate binding pocket is only available at either side of the membrane and a rigid body rocking motion is required for the conformational change between inward- and outward-facing conformations. B) In the two-gated pore model, however, the transmembrane pore contains a gate at either side at the membrane interfaces. The opening/closure of both gates are regulated by binding of the substrate/ions (Adapted from Figure 2B of (Focke et al., 2013)).

GltPh is proposed to comprise two structural domains: the 'trimerization' domain and the 'transport domain' (Reyes et al., 2009). Transition between the two conformations of the transporter involves a large movement of the transport domain, comprised of the substrate-binding transporter core and peripheral transmembrane (TM) segment 3 and 6, within the frame of the rigid trmierization domain (Reyes et al., 2009). This transition 
model of GltPh fits more to the rocker switch version. However, it has been proposed that binding and dissociation of the substrate and ions at either sides of the membrane is associated with additional conformation changes in some helical hairpins (HP) of the transporter. These HPs are referred to as the potential gates of GltPh (Huang and Tajkhorshid, 2008; Reyes et al., 2009), suggesting that a combination of both the twogated pore and the rocker switch is employed by the transporter.

Similar to GltPh, LeuT is proposed to possess two gates flanking the substrate and ion binding sites. These gates are asymmetric. While only a few residues comprise the extracellular gate of LeuT, referred to as the thin gate, the cytoplasmic gate is thick and made up of packed protein with a diameter of $\sim 20 \AA$ including 5 TMs (Krishnamurthy et al., 2009). This structural asymmetry is similar to GltPh which also has a thin extracellular gate (a few residues at the tip of its HP2) compared to its cytoplasmic gate, which is composed of HP1 and two of its TMs (Reyes et al., 2009). However, in contrast to GltPh, the core domain of LeuT does not move as a rigid body during the transition between the two conformations of a transport cycle, but only a portion of the core moves as a unit about a rotation axis which is oriented parallel to the membrane (Krishnamurthy and Gouaux, 2012). Therefore, this transporter does not involve a rocker switch mechanism. Moreover, the substrate/ion binding site is located at its center and is surrounded by four TMs (TM1, TM3, TM6 and TM8). Transition between the two conformations of LeuT involves approximately $37^{\circ}$ rotation of TM1 and TM6 relative to TM3 and TM8 (Krishnamurthy et al., 2009).

Together, the crystallographic data suggest that the transport mechanism of GltPh involves a combination of both a rocker switch and a two-gated pore mechanism for alternating access while LeuT seems to rely more on the two-gated pore mechanism (Focke et al., 2013).

If we speculate that the transport mechanisms of VGLUT and VGAT resemble that of GltPh and LeuT, respectively, this might explain the higher protein permeability of VGAT. With a combination of the rocker switch and two-gated pore mechanisms, more structural hindrances have to be overcome by protons to permeate than in case of only the two-gated mechanism. In other words, assuming that VGLUT acts like GltPh, a flow of protons through VGLUT would not only require opening of two gates but also a large movement of a core domain of the transporter which is needed for its conformational switch. Moreover, as will be discussed in section 4.5, it has been shown that even in the presence of substrate, $\mathrm{Cl}^{-}$is required to accelerate the transition between the two 
conformations of VGLUT (Preobraschenski et al., 2014). This makes VGLUT further less prone to conduct a leak flow of protons in the absence of glutamate and $\mathrm{Cl}^{-}$, and might explain the observed difference between these transporters to some extent.

However, this question that whether different transport mechanism of VGLUT and VGAT would affect their physiological role, remains unanswered. Considering that these transporters have similar substrate affinities (Omote and Moriyama, 2013), one possible answer would be that under physiological conditions where proton efflux is coupled to neurotransmitter import, a higher proton permeability of VGAT may lead to faster/more neurotransmitter loading and thus greater inhibitory quantal content. This might be particularly important in the context of homeostatic control of excitatory/inhibitory (E/I) synaptic strength. It has been shown that the expression levels of VGLUT and VGAT plays an important role in regulation of E/I synaptic plasticity by determining the quantal content (De Gois et al., 2005). Thus, a variation in quantal content due to differences in the transport activities of these transporters could grant additional significance to their role in controlling the E/I synaptic balance. However, further in vivo and in situ experiments are required to clarify whether and to what extent the measured differences in VGAT and VGLUT lead to changes in quantal content.

\subsection{VGAT Functions as a GABA/H+ Antiporter}

As mentioned above, the proton permeability of VGAT led to the hypothesis that VGAT functions as a GABA $/ \mathrm{H}^{+}$antiporter. Two findings strongly support this hypothesis. First, including $10 \mathrm{mM} \mathrm{GABA}$ in the alkalinizing buffer (see section 3.4) significantly accelerated the proton efflux from GABAergic SVs (Figure 3-14). Secondly, when GABA was added to acidified SVs, a drop in membrane potential was observed (Figure 3-27A) which due to the neutral charge of GABA molecules at pH 7.4, can only be attributed to a proton efflux from the lumen. This transport mechanism of VGAT was previously proposed in other studies where GABA transport was shown to require both components of $\Delta \mu_{H_{+}}$(Burger et al., 1991; Hell et al., 1990). Here a direct evidence for the coupling between GABA transport and proton efflux has been provided. However, this mechanism contradicts the work of Juge, et al. (2009) where VGAT was proposed to function as a $\mathrm{GABA} / \mathrm{Cl}^{-}$cotransporter. In this study, they showed that GABA uptake by proteoliposomes containing VGAT and the bacterial F-ATPase reached a maximum at $5 \mathrm{mM} \mathrm{Cl}^{-}$. Moreover, by means of uptake of radiolabeled $\mathrm{Cl}^{-}$, they showed that $\mathrm{Cl}^{-}$ 
transport was tightly coupled to GABA transport and that $\mathrm{Cl}^{-}$transport did not occur in the absence of GABA. These findings led to the proposal that during each transport cycle of VGAT, two $\mathrm{Cl}^{-}$ions and one GABA molecule are co-transported.

To test this hypothesis with the experimental system of this thesis, the effect of GABA on the membrane potential was measured in the presence of $5 \mathrm{mM} \mathrm{Cl}^{-}$in the bath solution. According to the proposed $\mathrm{GABA} / \mathrm{Cl}^{-}$co-transport stoichiometry (Juge et al., 2009), uptake of $10 \mathrm{mM}$ GABA would be associated with transport of $20 \mathrm{mM} \mathrm{Cl}^{-}$. Since the bath concentration of $\mathrm{Cl}^{-}$was $5 \mathrm{mM}$, this would result in Nernst potential of $\sim 35 \mathrm{mV}$. Therefore, if additional $\mathrm{Cl}^{-}$co-transport were to exist, uptake of $10 \mathrm{mM}$ GABA in the presence of $\mathrm{Cl}^{-}$would result in at least $\sim 35 \mathrm{mV}$ greater potential drop across the membrane of SVs compared to the absence of $\mathrm{Cl}^{-}$. In our measurements, GABA uptake in the presence of $5 \mathrm{mM}$ TEA-Cl in the bath solution resulted in $\sim 9 \mathrm{mV}$ greater potential drop across the membrane (Figure 3-27B), making a GABA/Cl- co-transport unlikely.

The observed slight increase in GABA-induced $\Delta \psi$ in the presence of $\mathrm{Cl}^{-}$can be attributed to the proposed enhanced GABA uptake in the presence of $\mathrm{Cl}^{-}$(Ahnert-Hilger and Jahn, 2011) which in turn would cause a larger proton efflux. With a direct transport of $\mathrm{Cl}^{-}$by VGAT, at least with the proposed stoichiometry (Juge et al., 2009) being unlikely, the stimulatory effect of $\mathrm{Cl}^{-}$on GABA uptake is probably a consequence of its effect on $\Delta \mu_{\mathrm{H}+}$.

Indeed, at $20-30 \mathrm{mM} \mathrm{Cl}^{-}$, which is estimated to be the concentration of $\mathrm{Cl}^{-}$at the presynaptic terminal (Price and Trussell, 2006), $\Delta \mu_{\mathrm{H}+}$ was shifted to a larger $\Delta \mathrm{pH}$ at the expense of $\Delta \psi$ (Figure 3-20 and Figure 3-22). $\mathrm{Cl}^{-}$transport into GABAergic SVs is most likely mediated by the $\mathrm{Cl}^{-} / \mathrm{H}^{+}$exchanger 3 (ClC3), as deletion of this exchanger has been shown to significantly impair $\mathrm{Cl}^{-}$induced acidification in GABAergic SVs (Riazanski et al., 2011). Moreover, it has been reported that loss of ClC3 leads to significant degeneration of the hippocampus and retina (Stobrawa et al., 2001), and this effect could be due to dysfunction of GABAergic interneurons (Ahnert-Hilger and Jahn, 2011). However, ClC7 (Gronborg et al., 2010) and VGLUT2 (Zander et al., 2010) also reside on GABAergic SVs and both of them may contribute to $\mathrm{Cl}^{-}$transport into these vesicles as well.

Together with the proposed GABA/ $\mathrm{H}^{+}$antiport mechanism, the effect of $\mathrm{Cl}^{-}$on $\Delta \mu_{\mathrm{H}+}$ explains how $\mathrm{Cl}^{-}$assists VGAT to transport thousands of GABA molecules into the lumen of SVs. During each transport cycle of VGAT, protons are shifted out in exchange for GABA, and $\mathrm{Cl}^{-}$influx provides the charge balance for the V-ATPase to pump more protons and thus allows for the maintenance of the $\mathrm{pH}$ gradient during transport. 
However, as will be discussed in the following sections, $\Delta \psi$ is also required for efficient GABA uptake, and GABAergic SVs must maintain the balance between the two components of $\Delta \mu_{\mathrm{H}+}$ by employing additional mechanisms, which convert $\Delta \mathrm{pH}$ back to $\Delta \psi$ and thereby prevent excessive acidification by $\mathrm{Cl}^{-}$.

\subsubsection{Regulation of Luminal Cl- in GABAergic SVs after Endocytosis}

As will be discussed in section 4.5, VGLUT in glutamatergic SVs can contribute to $\mathrm{Cl}^{-}$ efflux from the lumen of SVs in the early phase after endocytosis. With the abovediscussed data arguing against the permeability of VGAT to $\mathrm{Cl}^{-}$, the molecule responsible for $\mathrm{Cl}^{-}$efflux from endocytosed GABAergic SVs remains enigmatic. One candidate would be VGLUT2, which has been shown to reside on subset of GABAergic SVs (Zander et al., 2010). The other candidate would be one of the ClC isoforms (ClC3 and 7 (Gronborg et al., 2010)).

Since the intracellular ClCs (ClC3-7) are inaccessible for direct electrophysiological measurements, much of the current understanding of their function and biophysical characteristics has come from their heterologous plasma membrane overexpression (Leisle et al., 2011; Matsuda et al., 2010; Scheel et al., 2005; Smith and Lippiat, 2010). While it has been assumed that all intracellular ClCs exchange luminal protons for $\mathrm{Cl}^{-}$ (Jentsch, 2007), ClC4 and 5 have been proposed to directly acidify endosomes by exchanging cytosolic $\mathrm{H}^{+}$for the luminal $\mathrm{Cl}^{-}$shortly after they pinch off from the plasma membrane (Scheel et al., 2005). This view has also been supported recently where it was shown that ClC5 provides a mechanism for endosomal acidification distinct from $\mathrm{V}$ ATPase (Smith and Lippiat, 2010). It was proposed that this role of ClC5 might be restricted to early endocytosed vesicles with high $\mathrm{Cl}^{-}$concentration in the internalized fluid and an endosomal-negative transmembrane potential (Lippiat and Smith, 2012). Although no significant current at $\mathrm{pH} \sim 7.4$ was measured in HEK cells expressing ClC3 at the plasma membrane (Matsuda et al., 2010), overexpression of ClC7 in oocytes resulted in strong outward currents (Leisle et al., 2011). Moreover, it was demonstrated that in contrast to $\mathrm{ClC} 3, \mathrm{Cl}^{-}$exchange by $\mathrm{ClC} 7$ can occur in both directions at negative voltages (Leisle et al., 2011). This evidence opens up the possibility that ClC7, which has been detected on GABAergic SVs (Gronborg et al., 2010), contributes to the regulation of the luminal $\mathrm{Cl}^{-}$concentration at the initial phase after endocytosis. The ratio of $\mathrm{ClC7}$ protein in glutamatergic and GABAergic SVs could not be determined in previous 
proteomic analysis (Gronborg et al., 2010). Since this mechanism would be particularly beneficial to GABAergic SVs which have either no or lower copy number of VGLUT, it would be interesting to determine whether this protein is predominantly expressed on GABAergic SVs.

\subsubsection{Transport Mechanism of VGAT}

As mentioned earlier, detailed information is available about a eubacterial orthologue of the GABA transporters, LeuT, regarding the structural movements associated with the transport of ions and substrate (Focke et al., 2013; Krishnamurthy and Gouaux, 2012). If we speculate that the mechanism of transport of LeuT is similar to VGAT, we can extrapolate this structural framework to VGAT and propose a model for the GABA/H ${ }^{+}$ antiport mechanism.

According to this model, VGAT would possess at least two binding sites: one substrate binding site for GABA and one (or more than one) for proton(s). These biding sites would be located at the center of the transporter and would be flanked by two gates: a luminal gate (corresponding to the extracellular thin gate of LeuT (Krishnamurthy et al., 2009)) and a cytoplasmic gate. The net transport of substrate would require VGAT to switch from a conformation in which the cytoplasmic gate is open with the substrate binding site exposed to the cytoplasm, and the luminal gate is closed (state I) to a conformation in which the cytoplasmic gate is closed, one or more proton binding sites are exposed to the vesicular lumen and the luminal gate is open (state II) (Figure 4-2).

It has been proposed that upon binding of the substrate to LeuT, the conformational changes underlying closure of the extracellular gate largely disrupts the ion/substrate binding sites and leads to release of them to the cytoplasm (Krishnamurthy and Gouaux, 2012). This would suggest that in case of VGAT, closure of the cytoplasmic gate would disrupt the GABA binding site in state II, and the closure of the luminal gate might disrupt proton binding sites in state I. In other words, binding of GABA in state I closes the cytoplasmic gate, and induces structural changes which lead to the conformational switch to state II, and this in turn disrupts the GABA binding site. Thereby these structural changes would lead to a release of GABA into the lumen. This transport cycle would then be completed by binding of proton(s) to their binding sites on VGAT, which closes the luminal gate, reverses the structural changes and would again disrupt the proton binding site(s) at the cytoplasmic face. With disruption of proton binding sites at 
the cytoplasmic face, the transporter activity would not be affected by the cytoplasmic concentration of protons but rather would be strongly influenced by luminal $\mathrm{pH}$. This could explain the reported GABA uptake at pH 6.5 in the presence of $\mathrm{NH}^{+}{ }^{+}$where there was no $\mathrm{pH}$ gradient across the membrane but the lumen contained high proton concentration (Hell et al., 1990).

Based on this model, no more regulatory effectors than protons are required for efficient GABA transport. Moreover, in such a model both electrical and chemical components of $\Delta \mu_{\mathrm{H}+}$ by regulating proton interactions with its binding site(s) in VGAT can influence the conformational switch from state II to state I and thereby affect GABA transport.

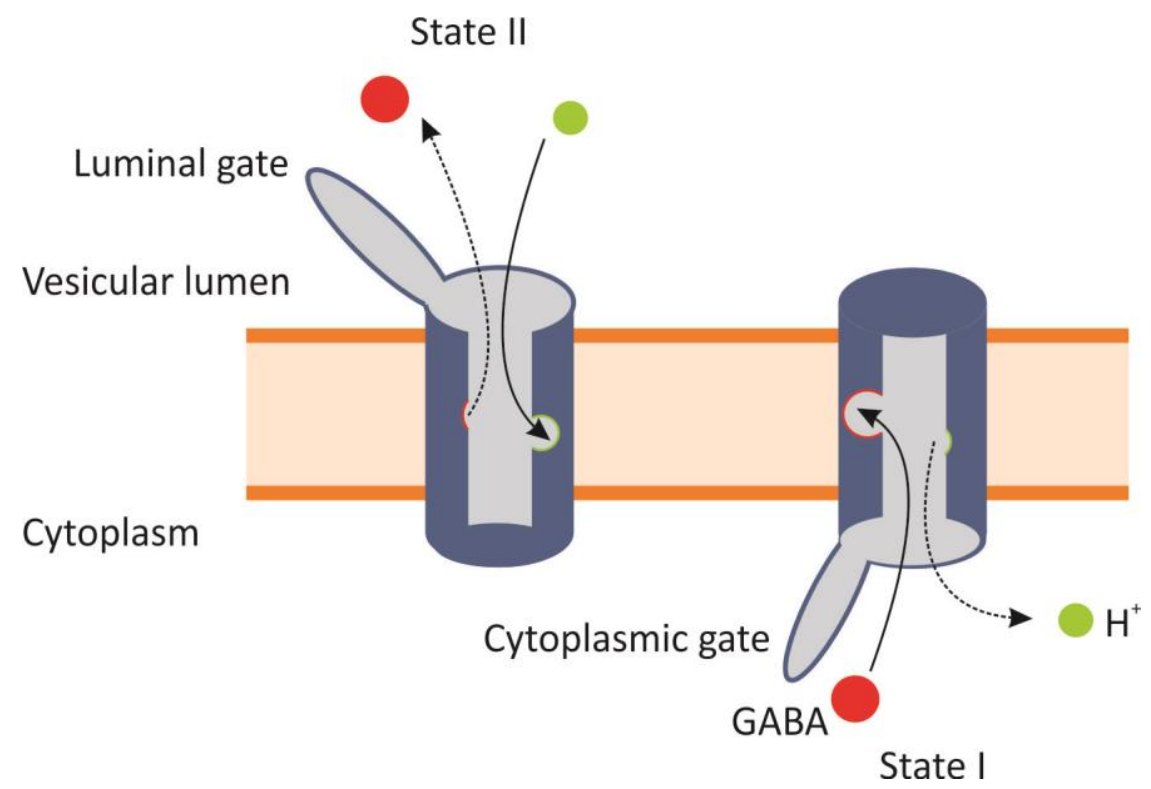

Figure 4-2 Model of GABA/H+ antiport by VGAT.

This model is proposed based on the two-gate pore mechanism of leucine transport by the eubacterial orthologue of the eukaryotic GABA transporters, LeuT. According to this mode, opening of the gates at each side of the membrane, disrupts one of the binding sites which would lead to release of the corresponding molecule/ion.

\subsection{VGLUT Transports Chloride and Potassium}

As discussed in section 3.5.2, the effect of $\mathrm{Cl}^{-}$on both components of $\Delta \mu_{\mathrm{H}+}$ was larger in glutamatergic compared to GABAergic SVs. Moreover, in the presence of $\mathrm{Cl}^{-}$, glutamatergic SVs acidified significantly faster than GABAergic SVs. Together, this implies higher $\mathrm{Cl}^{-}$influx in glutamatergic SVs. Since $\mathrm{ClC} 3$ is present on both vesicle subtypes in comparable quantities (Gronborg et al., 2010), it is reasonable to attribute 
the additional $\mathrm{Cl}^{-}$influx in glutamatergic SVs to VGLUT. This is in line with previous reports where the loss of VGLUT1 (Schenck et al., 2009), but not ClC3 (Riazanski et al., 2011; Schenck et al., 2009), significantly impaired $\mathrm{Cl}^{-}$induced acidification in brainpurified SVs, whose majority are glutamatergic. This notion has become quite controversial, with some supporting evidence (Bellocchio, 2000; Schenck et al., 2009) and some opposition (Hartinger and Jahn, 1993; Juge et al., 2006). Recently however, more direct evidence for $\mathrm{Cl}^{-}$conductance of VGLUT was provided by measuring $\mathrm{Cl}^{-}$ transport in VGLUT1-reconstituted liposomes using a $\mathrm{Cl}^{-}$-sensitive fluorescent probe (Preobraschenski et al., 2014). In this study, as described in section 1.2.2.3, VGLUT was proposed to possess two anion binding sites, one of which binds to $\mathrm{Cl}^{-}$and accelerate the conformational switch of the transporter and the second one preferentially binds to glutamate but can also be occupied by $\mathrm{Cl}^{-}$in the absence of glutamate (Preobraschenski et al., 2014). In this thesis, the measurements were also performed in the absence of glutamate. According to the proposed model, when $\mathrm{Cl}^{-}$is added to the bath solution, it binds to both the $\mathrm{Cl}^{-}$binding site and with lower affinity to the glutamate binding site, which the first facilitates the conformational change and the latter transports $\mathrm{Cl}^{-}$into the lumen. This can explain the observed greater $\mathrm{Cl}^{-}$influx in glutamatergic SVs in the absence of glutamate.

These results are consistent with the proposed model and demonstrate that the glutamate binding site of the VGLUT can indeed contributes to $\mathrm{Cl}^{-}$transport as well. This transport of $\mathrm{Cl}^{-}$but in the reverse direction, i.e. from the lumen to cytoplasm, would significantly facilitate glutamate transport under physiological conditions. At the early phase after endocytosis and during the first transport cycles of glutamate transport, the luminal concentration of $\mathrm{Cl}^{-}$is high while luminal glutamate is low. Thus, $\mathrm{Cl}^{-}$can bind to the glutamate binding site when VGLUT is in state II (i.e. the substrate binding pocket is towards the vesicular lumen) (Preobraschenski et al., 2014). This leads to efflux of $\mathrm{Cl}^{-}$ from the lumen of early-endocytosed vesicles and allows for the loading of the same concentration of glutamate without any net change in membrane potential, which would substantially enhance glutamate loading. Moreover, it helps to maintain the osmotic balance during vesicle filling. On the other hand, CIC3 can use the proton gradient established by V-ATPase to maintain luminal $\left[\mathrm{Cl}^{-}\right]$and thus allow VGLUT to continue $\mathrm{Cl}^{-}$ /glutamate exchange.

However, as discussed above, $\mathrm{Cl}^{-}$transport via $\mathrm{ClC} 3$ into the lumen of SVs shunts $\Delta \psi$, which would dissipate the main driving force for glutamate uptake. Therefore, another 
compensatory mechanism that tips the balance of the electrochemical components back towards $\Delta \psi$ would be very beneficial to neurotransmitter filling in glutamatergic SVs. Indeed, it has been shown that SVs can convert $\Delta \mathrm{pH}$ to $\Delta \psi$ via cation $/ \mathrm{H}^{+}$exchange mechanisms (Goh et al., 2011). In order to test whether such an exchange mechanism is particularly employed by glutamatergic SVs, one can compare the effect of $\mathrm{Na}^{+}$and $\mathrm{K}^{+}$on the $\Delta \mu_{\mathrm{H}+}$ in these vesicles with the response of GABAergic SVs, whose reliance on $\Delta \psi$ is significantly lower. In fact, as shown in Figure 3-17 and Figure 3-18, a significantly greater $\mathrm{K}^{+}$-induced alkalization was observed in glutamatergic compared to GABAergic SVs, which in contrast to $\mathrm{Na}^{+}$-induced alkalinization, was not blocked by the NHE inhibitor EIPA. The greater alkalinization of glutamatergic SVs by $\mathrm{K}^{+}$as well as its resistance to EIPA indicate that VGLUT is responsible for the $\mathrm{K}^{+}$transport into the lumen of SVs. This corroborates with the recently reported $\mathrm{K}^{+} / \mathrm{H}^{+}$exchange by VGLUT (Preobraschenski et al., 2014). Moreover, consistent with the measured effect of $\mathrm{K}^{+}$on $\Delta \psi$, it was observed that dissipation of the membrane potential upon glutamate uptake was significantly mitigated by the presence of $\mathrm{K}^{+}$(Figure $3-24 \mathrm{~B}$ ). This implies that stoichiometry of $\mathrm{K}^{+} / \mathrm{H}^{+}$exchange by VGLUT is not 1:1. Moreover, it further emphasizes the crucial role of VGLUT mediated $\mathrm{K}^{+} / \mathrm{H}^{+}$exchange as a charge compensating mechanism, which counteracts the effect of $\mathrm{Cl}^{-}$on the electrochemical gradient and helps to sustain the driving force required for efficient glutamate loading.

A slight $\mathrm{K}^{+}$effect was also observed in GABAergic SVs. Since it has been proposed that NHEs selectively transport $\mathrm{Na}^{+}$and not $\mathrm{K}^{+}$(Milosavljevic et al., 2014), and also due to the resistance of $\mathrm{K}^{+}$-induced alkalinization to the NHE inhibitor EIPA, this effect of $\mathrm{K}^{+}$in GABAergic SVs is probably mediated by VGLUT2 on a subset of these SVs (Zander et al., 2010).

\section{6 $\mathrm{Na}^{+} / \mathrm{H}^{+}$Exchange Stimulates Vesicle Loading in both Glutamatergic and GABAergic SVs}

$\mathrm{Na}^{+}$-induced alkalinization was measured to be equal in glutamatergic and GABAergic SVs (Figure 3-18), indicating that a common protein is mediating $\mathrm{Na}^{+}$influx. Since the effect of $\mathrm{Na}^{+}$was blocked by EIPA, an inhibitor of most NHE isoforms (Goh et al., 2011), and also a quantitative proteomics study revealed equal expression of NHEs on both vesicle subclasses (Gronborg et al., 2010), this $\mathrm{Na}^{+}$influx must be caused by NHEs, most probably NHE6 which resides on SVs (Preobraschenski et al., 2014). In case of 
glutamatergic SVs, which mainly rely on $\Delta \psi$ for neurotransmitter loading, $\mathrm{Na}^{+} / \mathrm{H}^{+}$ exchange by NHE6 in cooperation with $\mathrm{K}^{+} / \mathrm{H}^{+}$exchange by VGLUT stimulates neurotransmitter loading by maintaining $\Delta \psi$ at the expense of $\Delta \mathrm{pH}$. In addition, in GABAergic SVs, NHE6 counteracts the effect of $\mathrm{Cl}^{-}$on $\Delta \mu_{\mathrm{H}+}$ and together with ClC3 will provide a balance between $\Delta \mathrm{pH}$ and $\Delta \psi$, both of which are required by VGAT for efficient GABA loading.

Moreover, it has been recently proposed that NHE7, whose primary structure resembles that of NHE6 on SVs (Nakamura et al., 2005), can operate in a reverse mode, exchanging luminal $\mathrm{Na}^{+}$for cytosolic $\mathrm{H}^{+}$(Milosavljevic et al., 2014). This suggests that NHE6, in addition to its role as a regulator of $\Delta \mu_{\mathrm{H}+}$, might also contribute to $\mathrm{Na}^{+}$efflux from the lumen of early endocytosed SVs, which contain more than $100 \mathrm{mM} \mathrm{NaCl}$ in both glutamatergic and GABAergic SVs.

\subsection{Stoichiometry of $\mathrm{K}^{+} / \mathrm{H}^{+}$and $\mathrm{Na}^{+} / \mathrm{H}^{+}$Exchange}

In this thesis, changes of both components of $\Delta \mu_{\mathrm{H}+}$ in response to a known concentration of $\mathrm{K}^{+}$and $\mathrm{Na}^{+}$have been measured (section 3.5.1). As discussed above, assuming that VGLUT and NHE6 are responsible for the majority of $\mathrm{K}^{+}$and $\mathrm{Na}^{+}$influx, respectively, the stoichiometry of cation $/ \mathrm{H}^{+}$exchange by these transporters could be estimated as follows:

$\circ$ Using the measured relationship between the luminal $\mathrm{pH}$ and the buffering capacity (Figure 3-9), and the luminal pH of SVs before and after addition of 10 $\mathrm{mM} \mathrm{Na}^{+}$or $30 \mathrm{mM} \mathrm{K}^{+}$to the bath (Figure $3-15 \mathrm{~B}$ and $\mathrm{D}$ ), the averaged $\beta$ ( $\beta_{\text {Avg }}$ ) over this $\mathrm{pH}$ range was calculated.

- $\beta_{\text {Avg }}$ was multiplied by $\Delta \mathrm{pH}$ induced by addition of these cations to calculate the change in luminal concentration of protons $\left(\left[\mathrm{H}^{+}\right]_{\text {luminal }}\right)$, which was then converted to total number of protons per vesicle using the average volume of SVs (1.9 $\times 10-20$ L (Takamori et al., 2006)). This showed that 62 and 57 protons were exchanged for $10 \mathrm{mM} \mathrm{Na}^{+}$and $30 \mathrm{mM} \mathrm{K}^{+}$, respectively.

- Next, $\Delta \psi$ associated with the efflux of this amount of protons $\left(\Delta \psi_{[\mathrm{H}+]}\right)$ was calculated. Since changes in the luminal $\mathrm{pH}$ were in the range of $\mathrm{pH} 6.8-7.2, \Delta \psi_{[\mathrm{H}+]}$ was estimated from the $\Delta \mathrm{pH}-\Delta \psi$ correlation curve (Figure 3-6) by a linear fit to the range of $0.2-0.6 \Delta \mathrm{pH}$. 
○ $\Delta \psi_{[\mathrm{H}+]}$ was then added to the measured $\Delta \psi$ from the potentiometric assays upon the addition of $\mathrm{Na}^{+}$and $\mathrm{K}^{+}$. This yielded the total change induced by these cations in the membrane potential of SVs $\left(\Delta \psi_{\text {sum }}\right)$.

- $\Delta \psi_{\text {sum }}$ was then inserted into the Nernst equation to calculate the luminal concentration of the cations:

$$
\Delta \psi_{\text {sum }}=\frac{R T}{F} \times \ln \frac{[\text { Cation }]_{\text {luminal }}}{[\text { Cation }]_{\text {external }}}
$$

where $R$ is the universal gas constant, $T$ is the absolute temperature, $F$ is the Faraday constant and [Cation] external is equal to 10 and $30 \mathrm{mM}$ for $\mathrm{Na}^{+}$and $\mathrm{K}^{+}$, respectively. These calculations resulted in $5.5 \mathrm{mM}\left[\mathrm{Na}^{+}\right]_{\text {luminal }}$ and $17 \mathrm{mM}$

$\left[\mathrm{K}^{+}\right]_{\text {luminal, }}$ which were equivalent to $62 \mathrm{Na}^{+}$ions and $190 \mathrm{~K}^{+}$ions, respectively.

Together, this analysis revealed that 62 and $57 \mathrm{H}^{+}$were exchanged for $62 \mathrm{Na}^{+}$and 190 $\mathrm{K}^{+}$ions, respectively. Thus, it was estimated that NHE6 has a $\mathrm{H}^{+}: \mathrm{Na}^{+}$stoichiometry ratio of $1: 1$, which corroborates with the previously reported exchange ratio of NHEs (Bianchini and Poussegur, 1994), and VGLUT transports $\mathrm{K}^{+}$with a stoichiometry of 1:3 $\left(\mathrm{H}^{+}: \mathrm{K}^{+}\right)$, which promotes glutamate import due to its greater demand for $\Delta \psi$. This stoichiometry can explain the smaller glutamate-induced $\Delta \psi$ in the presence of $\mathrm{K}^{+}$ (Figure 3-24B).

\subsection{Neurotransmitters in the Lumen Stabilize $\Delta \mu_{H_{+}}$across the Membrane of SVs}

When $10 \mathrm{mM}$ of glutamate or GABA was included in the bath solution, the respective SVs reached significantly lower luminal pH. Two mechanisms can explain the lower luminal $\mathrm{pH}$ induced by neurotransmitter loading. First, dissipation of the membrane potential due to the negative charge of glutamate, similar to the $\mathrm{Cl}^{-}$effect described above, allows the V-ATPase to pump more protons and generates a larger $\mathrm{pH}$ gradient. Since GABA molecules are predominantly uncharged at neutral $\mathrm{pH}$, this mechanism is restricted to glutamate-induced acidification. This can explain why glutamate-induced acidification in glutamatergic SVs $(\Delta \mathrm{pH} \sim 0.4)$ exceeds acidification by GABA in GABAergic SVs $(\Delta \mathrm{pH} \sim 0.3)$. Secondly, it has been recently proposed that the glutamate effect on the luminal pH may also be due to the buffering effect of this molecule (Hnasko et al., 2010). Indeed, an additional $\sim 20 \mathrm{mM} / \Delta \mathrm{pH}$ buffering capacity was measured in the presence of glutamate (Figure 3-25), which is likely facilitated by the $\Upsilon$-carboxylic 
group ( $\mathrm{p} K_{\mathrm{a}} \sim 4.3$ ) of these molecules. Such scenario should hold true for GABA as well, as it possesses the same carboxylic group.

With the measured buffering effect, the luminal concentration of glutamate [Glu]luminal can be calculated using Eq. 4.3:

$$
\left[\mathrm{Glu}^{-}\right]_{\text {luminal }}=\frac{K_{a} \times\left[\mathrm{Glu}^{0}\right]}{\left[\mathrm{H}^{+}\right]_{\text {luminal }}}
$$

where $K_{\mathrm{a}}$ is the dissociation constant of the $\Upsilon$-carboxyl group of glutamate $(\sim 4.3)$, $\left[\mathrm{H}^{+}\right]_{\text {luminal }}$ is the luminal proton concentration in the presence of glutamate $\left(10^{-6.3}\right.$, see section 3.6.1), and $\left[\mathrm{Glu}^{0}\right]$ is the amount of protonated glutamate, which is equal to the amount of buffered protons and is determined by multiplying the additional $\beta$ (20 $\mathrm{mM} / \Delta \mathrm{pH}$ ) with the measured $\Delta \mathrm{pH}$. With this analysis, the luminal concentration of glutamate is estimated to be $\sim 400 \mathrm{mM}$. Previously, it was estimated that isolated SVs contain 100 mM glutamate (Burger et al., 1989; Hnasko et al., 2010). Two reasons could possibly explain this discrepancy. First, since pHluorin at $\mathrm{pH}$ around 6 is about $80 \%$ quenched, it cannot accurately report the $\mathrm{pH}$ changes in this low $\mathrm{pH}$ range, and thus the measured $\Delta \mathrm{pH}$ upon addition of methylamine in the presence of glutamate, might be underestimated. This in turn would result in an overestimation of $\beta$ (Eq. 2.2.) and subsequently of the luminal glutamate concentration. Secondly, the glutamate content has been previously measured in isolated SVs by HPLC (Burger et al., 1989). Although $\Delta \mu_{\mathrm{H}+}$ was preserved during isolation to retain the vesicular content, SVs might be damaged during homogenization, which would result in loss of glutamate during purification. Moreover, the actual value might be higher considering that a fraction of vesicles, and not the complete population, were glutamatergic.

The buffering effect of neurotransmitters helps the vesicles to maintain $\Delta \mu_{\mathrm{H}+}$ across the membrane upon vesicle loading. In fact, it was observed that upon blocking of the VATPase with bafilomycin, SVs which were acidified with glutamate lost their $\mathrm{pH}$ gradient much slower than those which were acidified to the same extent with $\mathrm{Cl}^{-}$(Hnasko et al., 2010). Therefore, the stimulatory effect of glutamate on the uptake of GABA and particularly monoamines, which predominantly relies on $\Delta \mathrm{pH}$ (Hnasko and Edwards, 2012), might also be due to its buffering effect which stabilizes the $\mathrm{pH}$ gradient across the membrane. 
It should be mentioned that there was also crosstalk between glutamatergic and GABAergic vesicles in terms of their neurotransmitter-induced acidification. Glutamateinduced acidification in GABAergic SVs is most likely due to the presence of other VGLUT isoforms, probably VGLUT2 (Zander et al., 2010), on these vesicles. This effect, referred to as vesicular synergy (El Mestikawy et al., 2011), has been proposed to improve GABA loading by enhancing the $\Delta \mathrm{pH}$ component of $\Delta \mu_{\mathrm{H}+}$. However, due to the very strict substrate specificity of VGLUTs (Omote and Moriyama, 2013), the effect of GABA in glutamatergic SVs cannot be attributed to its transport by VGLUTs. Moreover, although VGAT has been also found in glutamatergic synapses (Zander et al., 2010), there is no evidence that it resides on the same vesicles as VGLUT. Thus, the effect of GABA in glutamatergic SVs is probably due to non-specific leakage of GABA into the lumen of these vesicles. The cyclopentane or cyclopentene analogue of GABA in aqueous environments are more membrane permeable than the extended form of GABA (Burger et al., 1991; Crittenden et al., 2005), and may leak into the lumen of glutamatergic SVs. Nevertheless, glutamate-induced acidification in GABAergic SVs and GABA-induced acidification in glutamatergic SVs was significantly lower compared to their effect on their own vesicle types (Figure 3-23C and Figure 3-26C). 


\section{Major Findings and Outlook}

\subsection{Vesicular Transporters Significantly Contribute to Regulation of their Driving Force}

As mentioned above, proteomic analysis of glutamatergic and GABAergic SVs (Gronborg et al., 2010) revealed that these vesicles share the bulk of their constituents and none of the SV proteins other than vesicular transporters were exclusively found in one of the vesicle populations. However, some proteins were found to be preferentially expressed in either glutamatergic or GABAergic SVs, most of which play a role in SV trafficking. Among the proteins which were not involved in trafficking, significant differences were observed for SV2B, ZnT3 and SV2C with the first two proteins being more associated with glutamatergic SVs and the latter mainly found in GABAergic SVs.

The function of the SV2 proteins is not well understood. It is proposed that these proteins can interact with synaptotagmin and regulate $\mathrm{Ca}^{2+}{ }^{2+}$ stimulated SV exocytosis (Lazzell et al., 2004; Wang et al., 2003). In addition, they may play a role in osmotic buffering of neurotransmitters in the vesicular lumen (Reigada et al., 2003) and the regulation of the trafficking of the proteins to the vesicles through clathrin-mediated endocytosis (Morgans et al., 2009). However, no direct contribution of these proteins to neurotransmitter loading has been shown. The other protein $\mathrm{ZnT3}$ sequesters $\mathrm{Zn}^{2+}$ in a subset of glutamatergic SVs. It has been proposed that $\mathrm{Zn}^{2+}$ can be co-released with glutamate upon depolarization and modulates the activity of some glutamate receptors and transporters, among which a subset of NMDA receptors containing $\mathrm{Zn}^{2+}$-sensitive allosteric sites, are the most likely targets (Paoletti et al., 2009). Accumulation of $\mathrm{Zn}^{2+}$ in SVs would in principle affect $\Delta \mu_{\mathrm{H}+}$ in glutamatergic SVs due to its positive charge, however the assay buffers in this thesis were free of zinc. Although we cannot completely exclude the potential effect of other selectively enriched proteins to the generation or regulation of $\Delta \mu_{\mathrm{H}+}$, the differences in $\Delta \mu_{\mathrm{H}+}$ between glutamatergic and GABAergic SVs were primarily attributed to their vesicular transporters.

It was shown that there is indeed a difference in the magnitude of $\Delta \mu_{\mathrm{H}+}$ between glutamatergic and GABAergic SVs with glutamatergic vesicles having $17.79 \pm 6.9 \mathrm{mV}$ greater $\Delta \mu_{\mathrm{H}+}$. This difference was mainly due to the proton efflux through VGAT. It was demonstrated that VGAT functions as a $\mathrm{GABA} / \mathrm{H}^{+}$antiporter. This mechanism is 
beneficial to GABAergic SVs, as it does not disturb the balance between the two components of $\Delta \mu_{\mathrm{H}+}$. Moreover, it was shown that VGLUT significantly enhances its main driving force by two mechanisms. First, in early-endocytosed SVs it can transport out $\mathrm{Cl}^{-}$ during its glutamate transport cycle, which helps to prevent dissipation of membrane potential during the loading process. Secondly, it can function as a $\mathrm{K}^{+} / \mathrm{H}^{+}$exchanger, which converts the $\mathrm{pH}$ gradient to membrane potential. Together, these findings demonstrate that vesicular transporters along with other compensatory mechanisms can regulate the balance between the two components of the electrochemical gradient (Figure 5-1). In addition to their primary critical roles as neurotransmitter transporters, this adds yet another level of complexity to their contribution to the maintenance of fidelity in synaptic transmission.

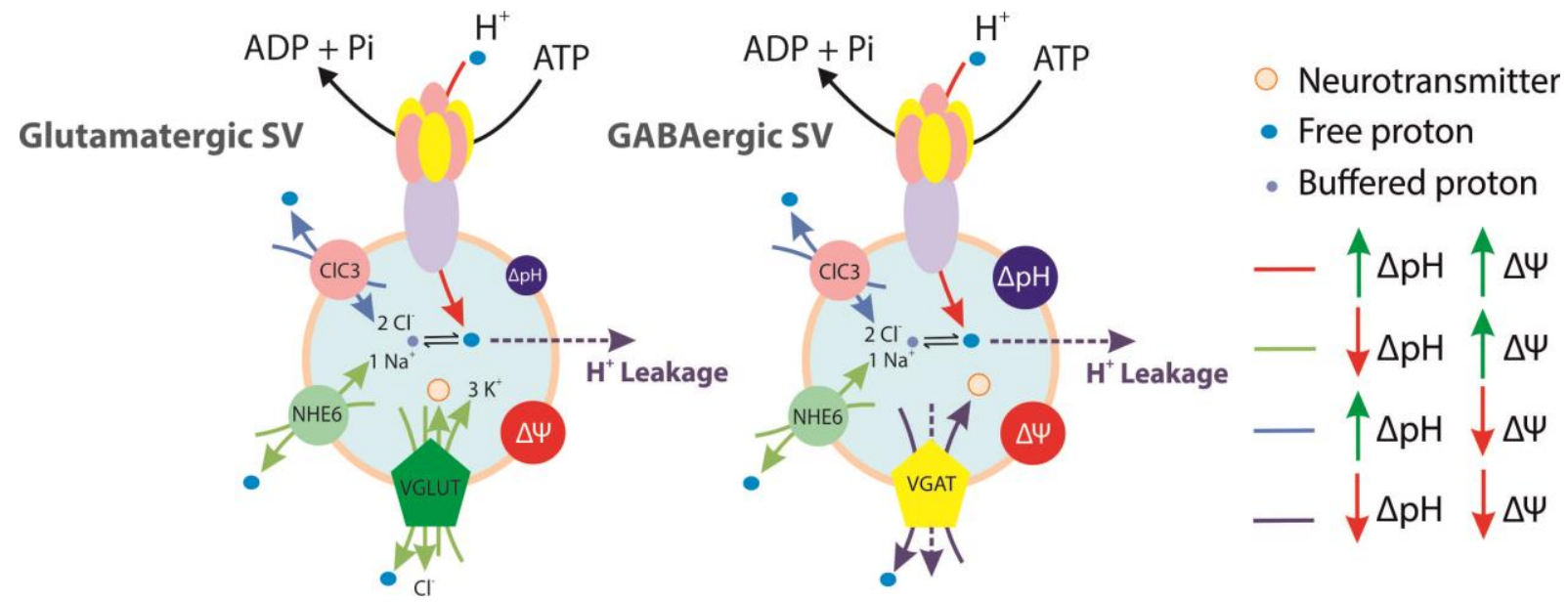

Figure 5-1 Regulation of the balance between the two components of $\Delta \mu_{\mathrm{H}+}$ in glutamatergic and GABAergic SVs.

A schematic depiction of the effect of all the ionic conductances, mediated by common vesicular ions exchangers (ClCs and NHEs) and vesicular neurotransmitter transporters, on the balance between the two components of $\Delta \mu_{\mathrm{H}+}$ in glutamatergic and GABAergic SVs. Arrows indicate the direction of ion flow across the membrane and their color represents their effect on the two components of $\Delta \mu_{\mathrm{H}_{+}}$as depicted in the color legend in the right panel.

\subsection{Single Vesicle Assay Reports Subtle Differences with high Precision}

The characteristics of the single vesicle assay grant it three main advantages over the conventional bulk assays. First, in the single vesicle assay the variation between different vesicle populations or within a population is studied under the same experimental condition. Such studies in bulk assay would require additional immune- 
isolation of vesicles and eventually the averaged response of each SV population would be measured. Second, in contrast to the bulk assays in which non-functional vesicles can weaken the measured signal, the single vesicle assay by detecting the response of each vesicle individually offers greater sensitivity. Third, the amount of vesicles required for single vesicle assay is lower. While 50-100 ng of SVs would suffice for a microscopybased assay, for bulk measurements usually 20-100 $\mu \mathrm{g}$ of vesicles are required to detect a decent signal.

In this thesis, the single vesicle assay was employed to unravel differences between

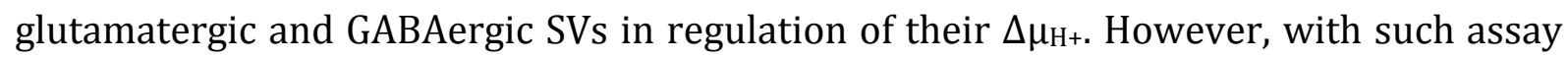
other open questions in the field of neurotransmission can also be addressed. For instance, it is known that both monoamines and acetylcholine are transported in exchange for 2 luminal protons by VMATs and VAchT, respectively, and thus rely mainly on $\Delta \mathrm{pH}$ (Johnson et al., 1981; Knoth et al., 1981; Nguyen et al., 1998). It has been shown that transport of anionic glutamate into aminergic (Hnasko et al., 2010) and cholinergic SVs (Gras et al., 2002) can enhance neurotransmitter loading into these SVs. However, not all the aminergic/cholinergic populations express VGLUTs (Hnasko and Edwards, 2012) and other regulatory mechanisms that would assist the transporters to load molar concentrations of these molecules into the vesicles (Edwards, 2007) are not well understood. Comparison of $\Delta \mu_{\mathrm{H}+}$ regulation between these SVs and glutamatergic SVs with much lower dependence on $\Delta \mathrm{pH}$ could provide valuable information about these regulatory mechanisms. The other potential application of single vesicle assay would be in studying the effect of a particular protein, which resides only on a subset of SVs, or a modification which is confined to a specific step of SV recycling on the regulation of $\Delta \mu_{\mathrm{H}+}$. For instance, by purifying clathrin-caoted SVs from the brain of transgenic mice expressing super-ecliptic pHluorin (Maycox et al., 1992) and labeling the clathrin-coated SVs with antibody against clathrin, one could compare the acidification extent and kinetics of coated SVs with uncoated vesicle and answer the question whether uncoating is a prerequisite for acidification and neurotransmitter loading (Milosevic et al., 2011).

However, there is still room for further optimizations in the described single vesicle assay. VF2.1.Cl and super-ecliptic pHluorin have overlapping excitation/emission spectra which hinder their simultaneous application. Therefore, red shifted potentiometric or pH-sensitive probes like pHTomato (Li and Tsien, 2012) would be beneficial to the assay. Moreover, there is no direct readout for the amount of loaded neurotransmitters in this assay. With recent advances in developing optical 
neurotransmitter sensors (Marvin et al., 2013), it would be possible to genetically target these probes to the luminal domain of SV proteins and measure single vesicle neurotransmitter uptake. 


\section{References}

Accardi, A., and Miller, C. (2004). Secondary active transport mediated by a prokaryotic homologue of ClC Cl- channels. Nature 427, 803-807.

Ahmed, S., Holt, M., Riedel, D., and Jahn, R. (2013). Small-scale isolation of synaptic vesicles from mammalian brain. Nature protocols 8, 998-1009.

Ahnert-Hilger, G., Holtje, M., Pahner, I., Winter, S., and Brunk, I. (2003). Regulation of vesicular neurotransmitter transporters. Reviews of physiology, biochemistry and pharmacology 150, 140-160.

Ahnert-Hilger, G., and Jahn, R. (2011). CLC-3 spices up GABAergic synaptic vesicles. Nature neuroscience 14, 405-407.

Aihara, Y., Mashima, H., Onda, H., Hisano, S., Kasuya, H., Hori, T., Yamada, S., Tomura, H., Yamada, Y., Inoue, I., et al. (2000). Molecular cloning of a novel brain-type $\mathrm{Na}(+)$-dependent inorganic phosphate cotransporter. Journal of neurochemistry 74, 2622-2625.

Alabi, A.A., and Tsien, R.W. (2013). Perspectives on kiss-and-run: role in exocytosis, endocytosis, and neurotransmission. Annual review of physiology 75, 393-422.

Amara, S.G. (1992). Neurotransmitter transporters. A tale of two families. Nature 360, 420-421.

Asada, H., Kawamura, Y., Maruyama, K., Kume, H., Ding, R.G., Kanbara, N., Kuzume, H., Sanbo, M., Yagi, T., and Obata, K. (1997). Cleft palate and decreased brain gamma-aminobutyric acid in mice lacking the 67-kDa isoform of glutamic acid decarboxylase. Proceedings of the National Academy of Sciences of the United States of America 94, 6496-6499.

Atluri, P.P., and Ryan, T.A. (2006). The kinetics of synaptic vesicle reacidification at hippocampal nerve terminals. The Journal of neuroscience : the official journal of the Society for Neuroscience 26, 2313-2320.

Bai, L., Xu, H., Collins, J.F., and Ghishan, F.K. (2001). Molecular and functional analysis of a novel neuronal vesicular glutamate transporter. The Journal of biological chemistry 276, 3676436769.

Bartoletti, T.M., and Thoreson, W.B. (2011). Quantal amplitude at the cone ribbon synapse can be adjusted by changes in cytosolic glutamate. Mol Vis 17, 920-931.

Bellocchio, E.E. (2000). Uptake of Glutamate into Synaptic Vesicles by an Inorganic Phosphate Transporter. Science 289, 957-960.

Bianchini, L., and Poussegur, J. (1994). Molecular structure and regulation of vertebrate $\mathrm{Na}+\mathrm{H}+$ exchangers. The Journal of experimental biology 196, 337-345.

Blakely, R.D., and Edwards, R.H. (2012). Vesicular and plasma membrane transporters for neurotransmitters. Cold Spring Harbor perspectives in biology 4 .

Brodal, P. (1992). The Central Nervous System: Structure and Function, Second edn (Oxford University Press, Inc.).

Budzinski, K.L., Zeigler, M., Fujimoto, B.S., Bajjalieh, S.M., and Chiu, D.T. (2011). Measurements of the acidification kinetics of single SynaptopHluorin vesicles. Biophysical journal 101, 1580-1589. 
Burger, P.M., Hell, J., Mehl, E., Krasel, C., Lottspeich, F., and Jahn, R. (1991). GABA and glycine in synaptic vesicles: storage and transport characteristics. Neuron 7, 287-293.

Burger, P.M., Mehl, E., Cameron, P.L., Maycox, P.R., Baumert, M., Lottspeich, F., De Camilli, P., and Jahn, R. (1989). Synaptic vesicles immunoisolated from rat cerebral cortex contain high levels of glutamate. Neuron 3, 715-720.

Carlson, M.D., Kish, P.E., and Ueda, T. (1989). Characterization of the Solubilized and Reconstituted Atp-Dependent Vesicular Glutamate Uptake System. Journal of Biological Chemistry 264, 7369-7376.

Chandy, G., Grabe, M., Moore, H.P., and Machen, T.E. (2001). Proton leak and CFTR in regulation of Golgi pH in respiratory epithelial cells. American journal of physiology Cell physiology 281, C908-921.

Chapman, E.R. (2008). How does synaptotagmin trigger neurotransmitter release? Annu Rev Biochem 77, 615-641.

Chaudhry, F.A., Reimer, R.J., and Edwards, R.H. (2002). The glutamine commute: take the N line and transfer to the A. The Journal of cell biology 157, 349-355.

Cidon, S., and Sihra, T.S. (1989). Characterization of a H+-ATPase in rat brain synaptic vesicles. Coupling to L-glutamate transport. The Journal of biological chemistry 264, 8281-8288.

Condie, B.G., Bain, G., Gottlieb, D.I., and Capecchi, M.R. (1997). Cleft palate in mice with a targeted mutation in the gamma-aminobutyric acid-producing enzyme glutamic acid decarboxylase 67. Proceedings of the National Academy of Sciences of the United States of America 94, 1145111455.

Crittenden, D.L., Chebib, M., and Jordan, M.J. (2005). Stabilization of zwitterions in solution: GABA analogues. The journal of physical chemistry A 109, 4195-4201.

Croft, B.G., Fortin, G.D., Corera, A.T., Edwards, R.H., Beaudet, A., Trudeau, L.E., and Fon, E.A. (2005). Normal biogenesis and cycling of empty synaptic vesicles in dopamine neurons of vesicular monoamine transporter 2 knockout mice. Molecular biology of the cell 16, 306-315.

Curthoys, N.P., and Watford, M. (1995). Regulation of glutaminase activity and glutamine metabolism. Annual review of nutrition 15, 133-159.

De Gois, S., Schafer, M.K., Defamie, N., Chen, C., Ricci, A., Weihe, E., Varoqui, H., and Erickson, J.D. (2005). Homeostatic scaling of vesicular glutamate and GABA transporter expression in rat neocortical circuits. The Journal of neuroscience : the official journal of the Society for Neuroscience 25, 7121-7133.

de Lange, R.P., de Roos, A.D., and Borst, J.G. (2003). Two modes of vesicle recycling in the rat calyx of Held. The Journal of neuroscience : the official journal of the Society for Neuroscience 23, 10164-10173.

Deng, L., Kaeser, P.S., Xu, W., and Sudhof, T.C. (2011). RIM proteins activate vesicle priming by reversing autoinhibitory homodimerization of Munc13. Neuron 69, 317-331.

Denker, A., Bethani, I., Krohnert, K., Korber, C., Horstmann, H., Wilhelm, B.G., Barysch, S.V., Kuner, T., Neher, E., and Rizzoli, S.O. (2011). A small pool of vesicles maintains synaptic activity in vivo. Proceedings of the National Academy of Sciences of the United States of America 108, 1717717182. 
Dittman, J., and Ryan, T.A. (2009). Molecular circuitry of endocytosis at nerve terminals. Annual review of cell and developmental biology 25, 133-160.

Edwards, R.H. (2007). The neurotransmitter cycle and quantal size. Neuron 55, 835-858.

Egashira, Y., Takase, M., and Takamori, S. (2015). Monitoring of vacuolar-type H+ ATPasemediated proton influx into synaptic vesicles. The Journal of neuroscience : the official journal of the Society for Neuroscience 35, 3701-3710.

Eiden, L.E., Schafer, M.K., Weihe, E., and Schutz, B. (2004). The vesicular amine transporter family (SLC18): amine/proton antiporters required for vesicular accumulation and regulated exocytotic secretion of monoamines and acetylcholine. Pflugers Archiv : European journal of physiology 447, 636-640.

Eisenberg, E., and Greene, L.E. (2007). Multiple roles of auxilin and hsc70 in clathrin-mediated endocytosis. Traffic 8, 640-646.

El Mestikawy, S., Wallen-Mackenzie, A., Fortin, G.M., Descarries, L., and Trudeau, L.E. (2011). From glutamate co-release to vesicular synergy: vesicular glutamate transporters. Nature reviews Neuroscience 12, 204-216.

Endresen, L.P., Hall, K., Hoye, J.S., and Myrheim, J. (2000). A theory for the membrane potential of living cells. European biophysics journal : EBJ 29, 90-103.

Fischer von Mollard, G., Sudhof, T.C., and Jahn, R. (1991). A small GTP-binding protein dissociates from synaptic vesicles during exocytosis. Nature $349,79-81$.

Focke, P.J., Wang, X., and Larsson, H.P. (2013). Neurotransmitter transporters: structure meets function. Structure 21, 694-705.

Fremeau, R.T., Jr., Burman, J., Qureshi, T., Tran, C.H., Proctor, J., Johnson, J., Zhang, H., Sulzer, D., Copenhagen, D.R., Storm-Mathisen, J., et al. (2002). The identification of vesicular glutamate transporter 3 suggests novel modes of signaling by glutamate. Proceedings of the National Academy of Sciences of the United States of America 99, 14488-14493.

Fremeau, R.T., Jr., Troyer, M.D., Pahner, I., Nygaard, G.O., Tran, C.H., Reimer, R.J., Bellocchio, E.E., Fortin, D., Storm-Mathisen, J., and Edwards, R.H. (2001). The expression of vesicular glutamate transporters defines two classes of excitatory synapse. Neuron 31, 247-260.

Gadd, J.C., Fujimoto, B.S., Bajjalieh, S.M., and Chiu, D.T. (2012). Single-molecule fluorescence quantification with a photobleached internal standard. Analytical chemistry 84, 10522-10525.

Gandhi, S.P., and Stevens, C.F. (2003). Three modes of synaptic vesicular recycling revealed by single-vesicle imaging. Nature 423, 607-613.

Gekle, M., and Silbernagl, S. (1995). Comparison of the buffer capacity of endocytotic vesicles, lysosomes and cytoplasm in cells derived from the proximal tubule of the kidney (opossum kidney cells). Pflugers Archiv : European journal of physiology 429, 452-454.

Goh, G.Y., Huang, H., Ullman, J., Borre, L., Hnasko, T.S., Trussell, L.O., and Edwards, R.H. (2011). Presynaptic regulation of quantal size: $\mathrm{K}+/ \mathrm{H}+$ exchange stimulates vesicular glutamate transport. Nature neuroscience 14, 1285-1292.

Goldsmith, D.J., and Hilton, P.J. (1992). Relationship between intracellular proton buffering capacity and intracellular pH. Kidney international 41, 43-49. 
Gouaux, E. (2009). Review. The molecular logic of sodium-coupled neurotransmitter transporters. Philosophical transactions of the Royal Society of London Series B, Biological sciences $364,149-154$.

Grabe, M., and Oster, G. (2001). Regulation of organelle acidity. The Journal of general physiology 117, 329-344.

Grabe, M., Wang, H., and Oster, G. (2000). The mechanochemistry of V-ATPase proton pumps. Biophysical journal 78, 2798-2813.

Granseth, B., and Lagnado, L. (2008). The role of endocytosis in regulating the strength of hippocampal synapses. The Journal of physiology 586, 5969-5982.

Granseth, B., Odermatt, B., Royle, S.J., and Lagnado, L. (2006). Clathrin-mediated endocytosis is the dominant mechanism of vesicle retrieval at hippocampal synapses. Neuron 51, 773-786.

Gras, C., Herzog, E., Bellenchi, G.C., Bernard, V., Ravassard, P., Pohl, M., Gasnier, B., Giros, B., and El Mestikawy, S. (2002). A third vesicular glutamate transporter expressed by cholinergic and serotoninergic neurons. The Journal of neuroscience : the official journal of the Society for Neuroscience 22, 5442-5451.

Gronborg, M., Pavlos, N.J., Brunk, I., Chua, J.J., Munster-Wandowski, A., Riedel, D., Ahnert-Hilger, G., Urlaub, H., and Jahn, R. (2010). Quantitative comparison of glutamatergic and GABAergic synaptic vesicles unveils selectivity for few proteins including MAL2, a novel synaptic vesicle protein. The Journal of neuroscience : the official journal of the Society for Neuroscience 30, 212.

Gundersen, V., Danbolt, N.C., Ottersen, O.P., and Storm-Mathisen, J. (1993). Demonstration of glutamate/aspartate uptake activity in nerve endings by use of antibodies recognizing exogenous D-aspartate. Neuroscience 57, 97-111.

Hallermann, S., Fejtova, A., Schmidt, H., Weyhersmuller, A., Silver, R.A., Gundelfinger, E.D., and Eilers, J. (2010). Bassoon Speeds Vesicle Reloading at a Central Excitatory Synapse. Neuron 68, 710-723.

Han, J., and Burgess, K. (2010). Fluorescent indicators for intracellular pH. Chemical reviews 110, 2709-2728.

Harata, N., Ryan, T.A., Smith, S.J., Buchanan, J., and Tsien, R.W. (2001). Visualizing recycling synaptic vesicles in hippocampal neurons by FM 1-43 photoconversion. Proceedings of the National Academy of Sciences of the United States of America 98, 12748-12753.

Hartinger, J., and Jahn, R. (1993). An Anion-Binding Site That Regulates the Glutamate Transporter of Synaptic Vesicles. Journal of Biological Chemistry 268, 23122-23127.

Haucke, V., Neher, E., and Sigrist, S.J. (2011). Protein scaffolds in the coupling of synaptic exocytosis and endocytosis. Nature reviews Neuroscience 12, 127-138.

Haugland, R.P. (2005). The Handbook: A Guide to Fluorescent Probes and Labeling Technologies, Tenth edn (Molecular Probes (invitrogen detection technologies)).

Hell, J.W., Maycox, P.R., and Jahn, R. (1990). Energy dependence and functional reconstitution of the gamma-aminobutyric acid carrier from synaptic vesicles. The Journal of biological chemistry 265, 2111-2117. 
Herman, M.A., Ackermann, F., Trimbuch, T., and Rosenmund, C. (2014). Vesicular glutamate transporter expression level affects synaptic vesicle release probability at hippocampal synapses in culture. The Journal of neuroscience : the official journal of the Society for Neuroscience 34, 11781-11791.

Herring, B.E., Silm, K., Edwards, R.H., and Nicoll, R.A. (2015). Is Aspartate an Excitatory Neurotransmitter? The Journal of neuroscience : the official journal of the Society for Neuroscience 35, 10168-10171.

Hertz, L., and Zielke, H.R. (2004). Astrocytic control of glutamatergic activity: astrocytes as stars of the show. Trends in neurosciences $27,735-743$.

Herzog, E., Bellenchi, G.C., Gras, C., Bernard, V., Ravassard, P., Bedet, C., Gasnier, B., Giros, B., and El Mestikawy, S. (2001). The existence of a second vesicular glutamate transporter specifies subpopulations of glutamatergic neurons. The Journal of neuroscience : the official journal of the Society for Neuroscience 21, RC181.

Hires, S.A., Zhu, Y., and Tsien, R.Y. (2008). Optical measurement of synaptic glutamate spillover and reuptake by linker optimized glutamate-sensitive fluorescent reporters. Proceedings of the National Academy of Sciences of the United States of America 105, 4411-4416.

Hnasko, T.S., Chuhma, N., Zhang, H., Goh, G.Y., Sulzer, D., Palmiter, R.D., Rayport, S., and Edwards, R.H. (2010). Vesicular glutamate transport promotes dopamine storage and glutamate corelease in vivo. Neuron 65, 643-656.

Hnasko, T.S., and Edwards, R.H. (2012). Neurotransmitter corelease: mechanism and physiological role. Annual review of physiology 74, 225-243.

Holt, M., Riedel, D., Stein, A., Schuette, C., and Jahn, R. (2008). Synaptic vesicles are constitutively active fusion machines that function independently of Ca2+. Current biology : CB 18, 715-722.

Hori, T., and Takahashi, T. (2012). Kinetics of synaptic vesicle refilling with neurotransmitter glutamate. Neuron 76, 511-517.

Huang, H., and Trussell, L.O. (2014). Presynaptic HCN channels regulate vesicular glutamate transport. Neuron 84, 340-346.

Huang, Z., and Tajkhorshid, E. (2008). Dynamics of the extracellular gate and ion-substrate coupling in the glutamate transporter. Biophysical journal 95, 2292-2300.

Huttner, W.B., Schiebler, W., Greengard, P., and De Camilli, P. (1983). Synapsin I (protein I), a nerve terminal-specific phosphoprotein. III. Its association with synaptic vesicles studied in a highly purified synaptic vesicle preparation. The Journal of cell biology 96, 1374-1388.

Ishikawa, T., Sahara, Y., and Takahashi, T. (2002). A single packet of transmitter does not saturate postsynaptic glutamate receptors. Neuron 34, 613-621.

Itoh, T., and De Camilli, P. (2006). BAR, F-BAR (EFC) and ENTH/ANTH domains in the regulation of membrane-cytosol interfaces and membrane curvature. Biochimica et biophysica acta 1761, 897-912.

Jahn, R., and Fasshauer, D. (2012). Molecular machines governing exocytosis of synaptic vesicles. Nature 490, 201-207.

Jentsch, T.J. (2007). Chloride and the endosomal-lysosomal pathway: emerging roles of CLC chloride transporters. The Journal of physiology 578, 633-640. 
Jentsch, T.J. (2008). CLC chloride channels and transporters: from genes to protein structure, pathology and physiology. Crit Rev Biochem Mol Biol 43, 3-36.

Johnson, R.G., Carty, S.E., and Scarpa, A. (1981). Proton: substrate stoichiometries during active transport of biogenic amines in chromaffin ghosts. The Journal of biological chemistry 256, 5773-5780.

Juge, N., Gray, J.A., Omote, H., Miyaji, T., Inoue, T., Hara, C., Uneyama, H., Edwards, R.H., Nicoll, R.A., and Moriyama, Y. (2010). Metabolic control of vesicular glutamate transport and release. Neuron 68, 99-112.

Juge, N., Muroyama, A., Hiasa, M., Omote, H., and Moriyama, Y. (2009). Vesicular inhibitory amino acid transporter is a Cl-/gamma-aminobutyrate Co-transporter. The Journal of biological chemistry $284,35073-35078$.

Juge, N., Yoshida, Y., Yatsushiro, S., Omote, H., and Moriyama, Y. (2006). Vesicular glutamate transporter contains two independent transport machineries. The Journal of biological chemistry 281, 39499-39506.

Jung, N., and Haucke, V. (2007). Clathrin-mediated endocytosis at synapses. Traffic 8, 1129-1136.

Kam, K., and Nicoll, R. (2007). Excitatory synaptic transmission persists independently of the glutamate-glutamine cycle. The Journal of neuroscience : the official journal of the Society for Neuroscience 27, 9192-9200.

Kanner, B.I., and Schuldiner, S. (1987). Mechanism of Transport and Storage of Neurotransmitter. Critical Reviews in Biochemistry and Molecular Biology 22, 1-38.

Kavalali, E.T. (2006). Synaptic vesicle reuse and its implications. The Neuroscientist : a review journal bringing neurobiology, neurology and psychiatry 12, 57-66.

Knoth, J., Zallakian, M., and Njus, D. (1981). Stoichiometry of H+-linked dopamine transport in chromaffin granule ghosts. Biochemistry 20, 6625-6629.

Korber, C., Horstmann, H., Satzler, K., and Kuner, T. (2012). Endocytic structures and synaptic vesicle recycling at a central synapse in awake rats. Traffic 13, 1601-1611.

Krishnamurthy, H., and Gouaux, E. (2012). X-ray structures of LeuT in substrate-free outwardopen and apo inward-open states. Nature 481, 469-474.

Krishnamurthy, H., Piscitelli, C.L., and Gouaux, E. (2009). Unlocking the molecular secrets of sodium-coupled transporters. Nature 459, 347-355.

Kvamme, E., Torgner, I.A., and Roberg, B. (2001). Kinetics and localization of brain phosphate activated glutaminase. J Neurosci Res 66, 951-958.

Kwon, S.E., and Chapman, E.R. (2011). Synaptophysin regulates the kinetics of synaptic vesicle endocytosis in central neurons. Neuron 70, 847-854.

Larsson, M., Sawada, K., Morland, C., Hiasa, M., Ormel, L., Moriyama, Y., and Gundersen, V. (2012). Functional and anatomical identification of a vesicular transporter mediating neuronal ATP release. Cerebral cortex 22, 1203-1214.

Lazzell, D.R., Belizaire, R., Thakur, P., Sherry, D.M., and Janz, R. (2004). SV2B regulates synaptotagmin 1 by direct interaction. The Journal of biological chemistry 279, 52124-52131. 
Leisle, L., Ludwig, C.F., Wagner, F.A., Jentsch, T.J., and Stauber, T. (2011). ClC-7 is a slowly voltage-gated $2 \mathrm{Cl}(-) / 1 \mathrm{H}(+)$-exchanger and requires 0stm 1 for transport activity. The EMBO journal 30, 2140-2152.

Li, Y., and Tsien, R.W. (2012). pHTomato, a red, genetically encoded indicator that enables multiplex interrogation of synaptic activity. Nature neuroscience 15, 1047-1053.

Li, Z., Burrone, J., Tyler, W.J., Hartman, K.N., Albeanu, D.F., and Murthy, V.N. (2005). Synaptic vesicle recycling studied in transgenic mice expressing synaptopHluorin. Proceedings of the National Academy of Sciences of the United States of America 102, 6131-6136.

Liang, S.L., Carlson, G.C., and Coulter, D.A. (2006). Dynamic regulation of synaptic GABA release by the glutamate-glutamine cycle in hippocampal area CA1. The Journal of neuroscience : the official journal of the Society for Neuroscience 26, 8537-8548.

Lippiat, J.D., and Smith, A.J. (2012). The CLC-5 2Cl(-)/H(+) exchange transporter in endosomal function and Dent's disease. Frontiers in physiology 3, 449.

Liu, G. (2003). Presynaptic control of quantal size: kinetic mechanisms and implications for synaptic transmission and plasticity. Current opinion in neurobiology 13, 324-331.

Ma, C., Su, L., Seven, A.B., Xu, Y., and Rizo, J. (2013). Reconstitution of the vital functions of Munc18 and Munc13 in neurotransmitter release. Science 339, 421-425.

Manente, S., De Pieri, S., Iero, A., Rigo, C., and Bragadin, M. (2008). A comparison between the responses of neutral red and acridine orange: acridine orange should be preferential and alternative to neutral red as a dye for the monitoring of contaminants by means of biological sensors. Analytical biochemistry 383, 316-319.

Maresova, L., Hoskova, B., Urbankova, E., Chaloupka, R., and Sychrova, H. (2010). New applications of pHluorin--measuring intracellular $\mathrm{pH}$ of prototrophic yeasts and determining changes in the buffering capacity of strains with affected potassium homeostasis. Yeast 27, 317325.

Maritzen, T., Podufall, J., and Haucke, V. (2010). Stonins--specialized adaptors for synaptic vesicle recycling and beyond? Traffic 11, 8-15.

Marra, V., Burden, J.J., Thorpe, J.R., Smith, I.T., Smith, S.L., Hausser, M., Branco, T., and Staras, K. (2012). A preferentially segregated recycling vesicle pool of limited size supports neurotransmission in native central synapses. Neuron 76, 579-589.

Marshansky, V., Rubinstein, J.L., and Gruber, G. (2014). Eukaryotic V-ATPase: novel structural findings and functional insights. Biochimica et biophysica acta 1837, 857-879.

Marvin, J.S., Borghuis, B.G., Tian, L., Cichon, J., Harnett, M.T., Akerboom, J., Gordus, A., Renninger, S.L., Chen, T.W., Bargmann, C.I., et al. (2013). An optimized fluorescent probe for visualizing glutamate neurotransmission. Nature methods 10, 162-170.

Masharina, A., Reymond, L., Maurel, D., Umezawa, K., and Johnsson, K. (2012). A fluorescent sensor for GABA and synthetic GABA(B) receptor ligands. Journal of the American Chemical Society $134,19026-19034$.

Masson, J., Sagne, C., Hamon, M., and El Mestikawy, S. (1999). Neurotransmitter transporters in the central nervous system. Pharmacological reviews 51, 439-464. 
Mathews, G.C., and Diamond, J.S. (2003). Neuronal glutamate uptake Contributes to GABA synthesis and inhibitory synaptic strength. The Journal of neuroscience : the official journal of the Society for Neuroscience 23, 2040-2048.

Matsuda, J.J., Filali, M.S., Collins, M.M., Volk, K.A., and Lamb, F.S. (2010). The ClC-3 Cl-/H+ antiporter becomes uncoupled at low extracellular $\mathrm{pH}$. The Journal of biological chemistry 285 , 2569-2579.

Maycox, P.R., Deckwerth, T., Hell, J.W., and Jahn, R. (1988). Glutamate uptake by brain synaptic vesicles. Energy dependence of transport and functional reconstitution in proteoliposomes. The Journal of biological chemistry 263, 15423-15428.

Maycox, P.R., Hell, J.W., and Jahn, R. (1990). Amino acid neurotransmission: spotlight on synaptic vesicles. Trends in neurosciences 13, 83-87.

Maycox, P.R., Link, E., Reetz, A., Morris, S.A., and Jahn, R. (1992). Clathrin-coated vesicles in nervous tissue are involved primarily in synaptic vesicle recycling. The Journal of cell biology $118,1379-1388$.

McCray, J.A., Herbette, L., Kihara, T., and Trentham, D.R. (1980). A new approach to timeresolved studies of ATP-requiring biological systems; laser flash photolysis of caged ATP. Proceedings of the National Academy of Sciences of the United States of America 77, 7237-7241.

McIntire, S.L., Reimer, R.J., Schuske, K., Edwards, R.H., and Jorgensen, E.M. (1997). Identification and characterization of the vesicular GABA transporter. Nature 389, 870-876.

McMahon, H.T., and Gallop, J.L. (2005). Membrane curvature and mechanisms of dynamic cell membrane remodelling. Nature 438, 590-596.

Miesenbock, G. (2012). Synapto-pHluorins: genetically encoded reporters of synaptic transmission. Cold Spring Harbor protocols 2012, 213-217.

Miesenbock, G., De Angelis, D.A., and Rothman, J.E. (1998). Visualizing secretion and synaptic transmission with pH-sensitive green fluorescent proteins. Nature 394, 192-195.

Miller, E.W., Lin, J.Y., Frady, E.P., Steinbach, P.A., Kristan, W.B., Jr., and Tsien, R.Y. (2012). Optically monitoring voltage in neurons by photo-induced electron transfer through molecular wires. Proceedings of the National Academy of Sciences of the United States of America 109, 2114-2119.

Milosavljevic, N., Monet, M., Lena, I., Brau, F., Lacas-Gervais, S., Feliciangeli, S., Counillon, L., and Poet, M. (2014). The intracellular $\mathrm{Na}(+) / \mathrm{H}(+)$ exchanger NHE7 effects a $\mathrm{Na}(+)$-coupled, but not $\mathrm{K}(+)$-coupled proton-loading mechanism in endocytosis. Cell reports 7, 689-696.

Milosevic, I., Giovedi, S., Lou, X., Raimondi, A., Collesi, C., Shen, H., Paradise, S., O'Toole, E., Ferguson, S., Cremona, O., et al. (2011). Recruitment of endophilin to clathrin-coated pit necks is required for efficient vesicle uncoating after fission. Neuron 72, 587-601.

Milovanovic, D., Honigmann, A., Koike, S., Gottfert, F., Pahler, G., Junius, M., Mullar, S., Diederichsen, U., Janshoff, A., Grubmuller, H., et al. (2015). Hydrophobic mismatch sorts SNARE proteins into distinct membrane domains. Nature communications 6.

Miyaji, T., Echigo, N., Hiasa, M., Senoh, S., Omote, H., and Moriyama, Y. (2008). Identification of a vesicular aspartate transporter. Proceedings of the National Academy of Sciences of the United States of America 105, 11720-11724. 
Moechars, D., Weston, M.C., Leo, S., Callaerts-Vegh, Z., Goris, I., Daneels, G., Buist, A., Cik, M., van der Spek, P., Kass, S., et al. (2006). Vesicular glutamate transporter VGLUT2 expression levels control quantal size and neuropathic pain. The Journal of neuroscience : the official journal of the Society for Neuroscience 26, 12055-12066.

Morgans, C.W., Kensel-Hammes, P., Hurley, J.B., Burton, K., Idzerda, R., McKnight, G.S., and Bajjalieh, S.M. (2009). Loss of the Synaptic Vesicle Protein SV2B results in reduced neurotransmission and altered synaptic vesicle protein expression in the retina. PloS one 4, e5230.

Moriyama, Y., Maeda, M., and Futai, M. (1990). Energy coupling of L-glutamate transport and vacuolar $\mathrm{H}(+)$-ATPase in brain synaptic vesicles. Journal of biochemistry 108, 689-693.

Moriyama, Y., Maeda, M., and Futai, M. (1992). Involvement of a non-proton pump factor (possibly Donnan-type equilibrium) in maintenance of an acidic $\mathrm{pH}$ in lysosomes. FEBS letters 302, 18-20.

Moriyama, Y., and Yamamoto, A. (1995). Vesicular L-glutamate transporter in microvesicles from bovine pineal glands. Driving force, mechanism of chloride anion activation, and substrate specificity. The Journal of biological chemistry 270, 22314-22320.

Morland, C., Nordengen, K., Larsson, M., Prolo, L.M., Farzampour, Z., Reimer, R.J., and Gundersen, V. (2013). Vesicular uptake and exocytosis of L-aspartate is independent of sialin. FASEB journal : official publication of the Federation of American Societies for Experimental Biology 27, 12641274.

Mortensen, O.V., and Amara, S.G. (2003). Dynamic regulation of the dopamine transporter. European journal of pharmacology 479, 159-170.

Nagy, A., Baker, R.R., Morris, S.J., and Whittaker, V.P. (1976). The preparation and characterization of synaptic vesicles of high purity. Brain research 109, 285-309.

Naito, S., and Ueda, T. (1985). Characterization of Glutamate Uptake into Synaptic Vesicles. Journal of neurochemistry 44, 99-109.

Nakamura, N., Tanaka, S., Teko, Y., Mitsui, K., and Kanazawa, H. (2005). Four Na+/H+ exchanger isoforms are distributed to Golgi and post-Golgi compartments and are involved in organelle $\mathrm{pH}$ regulation. The Journal of biological chemistry 280, 1561-1572.

Neher, E. (2010). What is Rate-Limiting during Sustained Synaptic Activity: Vesicle Supply or the Availability of Release Sites. Frontiers in synaptic neuroscience 2, 144.

Nguyen, M.L., Cox, G.D., and Parsons, S.M. (1998). Kinetic parameters for the vesicular acetylcholine transporter: two protons are exchanged for one acetylcholine. Biochemistry 37, 13400-13410.

Ni, B., Rosteck, P.R., Jr., Nadi, N.S., and Paul, S.M. (1994). Cloning and expression of a cDNA encoding a brain-specific $\mathrm{Na}(+)$-dependent inorganic phosphate cotransporter. Proceedings of the National Academy of Sciences of the United States of America 91, 5607-5611.

Okumoto, S., Looger, L.L., Micheva, K.D., Reimer, R.J., Smith, S.J., and Frommer, W.B. (2005). Detection of glutamate release from neurons by genetically encoded surface-displayed FRET nanosensors. Proceedings of the National Academy of Sciences of the United States of America $102,8740-8745$. 
Olivo-Marin, J.C. (2002). Extraction of spots in biological images using multiscale products. Pattern Recognition 35, 1989-1996.

Omote, H., Miyaji, T., Juge, N., and Moriyama, Y. (2011). Vesicular neurotransmitter transporter: bioenergetics and regulation of glutamate transport. Biochemistry 50, 5558-5565.

Omote, H., and Moriyama, Y. (2013). Vesicular neurotransmitter transporters: an approach for studying transporters with purified proteins. Physiology 28, 39-50.

Orlowski, J., and Grinstein, S. (2004). Diversity of the mammalian sodium/proton exchanger SLC9 gene family. Pflugers Archiv : European journal of physiology 447, 549-565.

Overly, C.C., Lee, K.D., Berthiaume, E., and Hollenbeck, P.J. (1995). Quantitative measurement of intraorganelle $\mathrm{pH}$ in the endosomal-lysosomal pathway in neurons by using ratiometric imaging with pyranine. Proceedings of the National Academy of Sciences of the United States of America 92, 3156-3160.

Palmgren, M.G. (1991). Acridine-Orange as a Probe for Measuring Ph Gradients across Membranes - Mechanism and Limitations. Analytical biochemistry 192, 316-321.

Paoletti, P., Vergnano, A.M., Barbour, B., and Casado, M. (2009). Zinc at glutamatergic synapses. Neuroscience 158, 126-136.

Parsons, R.L., Calupca, M.A., Merriam, L.A., and Prior, C. (1999). Empty synaptic vesicles recycle and undergo exocytosis at vesamicol-treated motor nerve terminals. Journal of neurophysiology 81, 2696-2700.

Pereda, A.E. (2014). Electrical synapses and their functional interactions with chemical synapses. Nature Reviews Neuroscience 15, 250-263.

Perez-Sayans, M., Suarez-Penaranda, J.M., Barros-Angueira, F., Diz, P.G., Gandara-Rey, J.M., and Garcia-Garcia, A. (2012). An update in the structure, function, and regulation of V-ATPases: the role of the C subunit. Brazilian journal of biology = Revista brasleira de biologia 72, 189-198.

Preobraschenski, J., Zander, J.F., Suzuki, T., Ahnert-Hilger, G., and Jahn, R. (2014). Vesicular glutamate transporters use flexible anion and cation binding sites for efficient accumulation of neurotransmitter. Neuron 84, 1287-1301.

Price, G.D., and Trussell, L.O. (2006). Estimate of the chloride concentration in a central glutamatergic terminal: a gramicidin perforated-patch study on the calyx of Held. The Journal of neuroscience : the official journal of the Society for Neuroscience 26,11432-11436.

Purves, D. (2012). Neuroscience, Fifth edn (Sinauer Associates, Inc.).

Pyle, J.L., Kavalali, E.T., Piedras-Renteria, E.S., and Tsien, R.W. (2000). Rapid reuse of readily releasable pool vesicles at hippocampal synapses. Neuron 28, 221-231.

Qiu, X., Zhu, Q., and Sun, J. (2015). Quantitative analysis of vesicle recycling at the calyx of Held synapse. Proceedings of the National Academy of Sciences of the United States of America 112, 4779-4784.

Rangaraju, V., Calloway, N., and Ryan, T.A. (2014). Activity-driven local ATP synthesis is required for synaptic function. Cell 156, 825-835.

Reigada, D., Diez-Perez, I., Gorostiza, P., Verdaguer, A., Gomez de Aranda, I., Pineda, O., Vilarrasa, J., Marsal, J., Blasi, J., Aleu, J., et al. (2003). Control of neurotransmitter release by an internal gel 
matrix in synaptic vesicles. Proceedings of the National Academy of Sciences of the United States of America 100, 3485-3490.

Reimer, R.J. (2013). SLC17: a functionally diverse family of organic anion transporters. Molecular aspects of medicine $34,350-359$.

Reimer, R.J., and Edwards, R.H. (2004). Organic anion transport is the primary function of the SLC17/type I phosphate transporter family. Pflugers Archiv : European journal of physiology 447, 629-635.

Reyes, N., Ginter, C., and Boudker, O. (2009). Transport mechanism of a bacterial homologue of glutamate transporters. Nature 462, 880-885.

Riazanski, V., Deriy, L.V., Shevchenko, P.D., Le, B., Gomez, E.A., and Nelson, D.J. (2011). Presynaptic CLC-3 determines quantal size of inhibitory transmission in the hippocampus. Nature neuroscience 14, 487-494.

Richards, D.A., Guatimosim, C., Rizzoli, S.0., and Betz, W.J. (2003). Synaptic Vesicle Pools at the Frog Neuromuscular Junction. Neuron 39, 529-541.

Ritchie, R.J., and Gibson, J. (1987). Permeability of Ammonia, Methylamine and Ethylamine in the Cyanobacterium, Synechococcus R-2 (Anacystis-Nidulans) Pcc-7942. J Membrane Biol 95, 131142.

Rizzoli, S.O., Bethani, I., Zwilling, D., Wenzel, D., Siddiqui, T.J., Brandhorst, D., and Jahn, R. (2006). Evidence for Early Endosome-like Fusion of Recently Endocytosed Synaptic Vesicles. Traffic 7, 1163-1176.

Roberts, R.C., Roche, J.K., and McCullumsmith, R.E. (2014). Localization of excitatory amino acid transporters EAAT1 and EAAT2 in human postmortem cortex: a light and electron microscopic study. Neuroscience 277, 522-540.

Rottenberg, H., and Moreno-Sanchez, R. (1993). The proton pumping activity of H(+)-ATPases: an improved fluorescence assay. Biochimica et biophysica acta 1183, 161-170.

Roux, A., Uyhazi, K., Frost, A., and De Camilli, P. (2006). GTP-dependent twisting of dynamin implicates constriction and tension in membrane fission. Nature 441, 528-531.

Rybak, S.L., Lanni, F., and Murphy, R.F. (1997). Theoretical considerations on the role of membrane potential in the regulation of endosomal pH. Biophysical journal 73, 674-687.

Saheki, Y., and De Camilli, P. (2012). Synaptic vesicle endocytosis. Cold Spring Harbor perspectives in biology 4, a005645.

Saleh, A.M., and Batlle, D.C. (1990). Kinetic properties of the Na+/H+ antiporter of lymphocytes from the spontaneously hypertensive rat: role of intracellular $\mathrm{pH}$. The Journal of clinical investigation $85,1734-1739$.

Sankaranarayanan, S., De Angelis, D., Rothman, J.E., and Ryan, T.A. (2000). The use of pHluorins for optical measurements of presynaptic activity. Biophysical journal 79, 2199-2208.

Sara, Y., Mozhayeva, M.G., Liu, X., and Kavalali, E.T. (2002). Fast vesicle recycling supports neurotransmission during sustained stimulation at hippocampal synapses. The Journal of neuroscience : the official journal of the Society for Neuroscience 22, 1608-1617. 
Sawada, K., Echigo, N., Juge, N., Miyaji, T., Otsuka, M., Omote, H., Yamamoto, A., and Moriyama, Y. (2008). Identification of a vesicular nucleotide transporter. Proceedings of the National Academy of Sciences of the United States of America 105, 5683-5686.

Schafer, M.K., Varoqui, H., Defamie, N., Weihe, E., and Erickson, J.D. (2002). Molecular cloning and functional identification of mouse vesicular glutamate transporter 3 and its expression in subsets of novel excitatory neurons. The Journal of biological chemistry $277,50734-50748$.

Schagger, H. (2006). Tricine-SDS-PAGE. Nature protocols 1, 16-22.

Scheel, O., Zdebik, A.A., Lourdel, S., and Jentsch, T.J. (2005). Voltage-dependent electrogenic chloride/proton exchange by endosomal CLC proteins. Nature 436, 424-427.

Schenck, S., Wojcik, S.M., Brose, N., and Takamori, S. (2009). A chloride conductance in VGLUT1 underlies maximal glutamate loading into synaptic vesicles. Nature neuroscience 12, 156-162.

Shapiro, H.M. (2000). Membrane potential estimation by flow cytometry. Methods 21, 271-279.

Sheng, M., and Kim, M.J. (2002). Postsynaptic signaling and plasticity mechanisms. Science 298, 776-780.

Smith, A.J., and Lippiat, J.D. (2010). Direct endosomal acidification by the outwardly rectifying CLC-5 Cl-/H+exchanger. The Journal of physiology 588, 2033-2045.

Song, H., Ming, G., Fon, E., Bellocchio, E., Edwards, R.H., and Poo, M. (1997). Expression of a putative vesicular acetylcholine transporter facilitates quantal transmitter packaging. Neuron $18,815-826$.

Stobrawa, S.M., Breiderhoff, T., Takamori, S., Engel, D., Schweizer, M., Zdebik, A.A., Bosl, M.R., Ruether, K., Jahn, H., Draguhn, A., et al. (2001). Disruption of ClC-3, a chloride channel expressed on synaptic vesicles, leads to a loss of the hippocampus. Neuron 29, 185-196.

Südhof, T.C. (2004). The synaptic vesicle cycle. Annual review of neuroscience 27, 509-547.

Südhof, T.C., and Rizo, J. (2011). Synaptic vesicle exocytosis. Cold Spring Harbor perspectives in biology 3.

Sun-Wada, G.H., Murata, Y., Namba, M., Yamamoto, A., Wada, Y., and Futai, M. (2003). Mouse proton pump ATPase $\mathrm{C}$ subunit isoforms (C2-a and C2-b) specifically expressed in kidney and lung. The Journal of biological chemistry $278,44843-44851$.

Szatkowski, M.S., and Thomas, R.C. (1989). The intrinsic intracellular H+ buffering power of snail neurones. The Journal of physiology 409, 89-101.

Tabares, L., Ales, E., Lindau, M., and Alvarez de Toledo, G. (2001). Exocytosis of catecholamine (CA)-containing and CA-free granules in chromaffin cells. The Journal of biological chemistry 276, 39974-39979.

Takamori, S., Holt, M., Stenius, K., Lemke, E.A., Gronborg, M., Riedel, D., Urlaub, H., Schenck, S., Brugger, B., Ringler, P., et al. (2006). Molecular anatomy of a trafficking organelle. Cell 127, 831846.

Takamori, S., Malherbe, P., Broger, C., and Jahn, R. (2002). Molecular cloning and functional characterization of human vesicular glutamate transporter 3. EMBO reports 3, 798-803. 
Takamori, S., Rhee, J.S., Rosenmund, C., and Jahn, R. (2001). Identification of differentiationassociated brain-specific phosphate transporter as a second vesicular glutamate transporter (VGLUT2). The Journal of neuroscience : the official journal of the Society for Neuroscience 21, RC182.

Takamori, S., Riedel, D., and Jahn, R. (2000). Immunoisolation of GABA-specific synaptic vesicles defines a functionally distinct subset of synaptic vesicles. The Journal of neuroscience : the official journal of the Society for Neuroscience 20, 4904-4911.

Toei, M., Saum, R., and Forgac, M. (2010). Regulation and isoform function of the V-ATPases. Biochemistry 49, 4715-4723.

Towbin, H., Staehelin, T., and Gordon, J. (1989). Immunoblotting in the clinical laboratory. Journal of clinical chemistry and clinical biochemistry Zeitschrift fur klinische Chemie und klinische Biochemie 27, 495-501.

Van Dyke, R.W. (1993). Acidification of rat liver lysosomes: quantitation and comparison with endosomes. The American journal of physiology 265, C901-917.

Van Dyke, R.W., and Belcher, J.D. (1994). Acidification of three types of liver endocytic vesicles: similarities and differences. The American journal of physiology 266, C81-94.

Varoqueaux, F., Sigler, A., Rhee, J.S., Brose, N., Enk, C., Reim, K., and Rosenmund, C. (2002). Total arrest of spontaneous and evoked synaptic transmission but normal synaptogenesis in the absence of Munc13-mediated vesicle priming. Proceedings of the National Academy of Sciences of the United States of America 99, 9037-9042.

Varoqui, H., Schafer, M.K., Zhu, H., Weihe, E., and Erickson, J.D. (2002). Identification of the differentiation-associated $\mathrm{Na}+\mathrm{PI}$ transporter as a novel vesicular glutamate transporter expressed in a distinct set of glutamatergic synapses. The Journal of neuroscience : the official journal of the Society for Neuroscience 22, 142-155.

Vaughan-Jones, R.D., and Wu, M.L. (1990). pH dependence of intrinsic H+ buffering power in the sheep cardiac Purkinje fibre. The Journal of physiology 425, 429-448.

Verhage, M., Maia, A.S., Plomp, J.J., Brussaard, A.B., Heeroma, J.H., Vermeer, H., Toonen, R.F., Hammer, R.E., van den Berg, T.K., Missler, M., et al. (2000). Synaptic assembly of the brain in the absence of neurotransmitter secretion. Science 287, 864-869.

Verhage, M., and Sorensen, J.B. (2008). Vesicle docking in regulated exocytosis. Traffic 9, 14141424.

Walker, J.W., Reid, Gordon P, Mccray, James A, Trentham, David R (1988). Photolabile 1 - ( 2Nitropheny1 ) ethyl Phosphate Esters of Adenine Nucleotide Analogues. Synthesis and Mechanism of Photolysis. American Chemical Society 110, 7170-7177.

Wan, F.Y., Wang, Y.N., and Zhang, G.J. (2002). Influence of the physical states of membrane surface area and center area on lysosomal proton permeability. Archives of biochemistry and biophysics 404, 285-292.

Wang, L., Tu, P., Bonet, L., Aubrey, K.R., and Supplisson, S. (2013). Cytosolic transmitter concentration regulates vesicle cycling at hippocampal GABAergic terminals. Neuron 80, 143158. 
Wang, M.M., Janz, R., Belizaire, R., Frishman, L.J., and Sherry, D.M. (2003). Differential distribution and developmental expression of synaptic vesicle protein 2 isoforms in the mouse retina. The Journal of comparative neurology 460, 106-122.

Wang, Z.Q., and Gluck, S. (1990). Isolation and properties of bovine kidney brush border vacuolar $\mathrm{H}(+)$-ATPase. A proton pump with enzymatic and structural differences from kidney microsomal H(+)-ATPase. The Journal of biological chemistry 265, 21957-21965.

Watanabe, S., Liu, Q., Davis, M.W., Hollopeter, G., Thomas, N., Jorgensen, N.B., and Jorgensen, E.M. (2013a). Ultrafast endocytosis at Caenorhabditis elegans neuromuscular junctions. eLife 2.

Watanabe, S., Rost, B.R., Camacho-Perez, M., Davis, M.W., Sohl-Kielczynski, B., Rosenmund, C., and Jorgensen, E.M. (2013b). Ultrafast endocytosis at mouse hippocampal synapses. Nature 504, 242-247.

Wei, J., and Wu, J.Y. (2008). Post-translational regulation of L-glutamic acid decarboxylase in the brain. Neurochemical research 33, 1459-1465.

Werner, A., Moore, M.L., Mantei, N., Biber, J., Semenza, G., and Murer, H. (1991). Cloning and expression of cDNA for a $\mathrm{Na} / \mathrm{Pi}$ cotransport system of kidney cortex. Proceedings of the National Academy of Sciences of the United States of America 88, 9608-9612.

Wilson, N.R., Kang, J., Hueske, E.V., Leung, T., Varoqui, H., Murnick, J.G., Erickson, J.D., and Liu, G. (2005). Presynaptic regulation of quantal size by the vesicular glutamate transporter VGLUT1. The Journal of neuroscience : the official journal of the Society for Neuroscience 25, 6221-6234.

Wojcik, S.M., Katsurabayashi, S., Guillemin, I., Friauf, E., Rosenmund, C., Brose, N., and Rhee, J.S. (2006). A shared vesicular carrier allows synaptic corelease of GABA and glycine. Neuron 50, 575-587.

Wojcik, S.M., Rhee, J.S., Herzog, E., Sigler, A., Jahn, R., Takamori, S., Brose, N., and Rosenmund, C. (2004). An essential role for vesicular glutamate transporter 1 (VGLUT1) in postnatal development and control of quantal size. Proceedings of the National Academy of Sciences of the United States of America 101, 7158-7163.

Wolosker, H., deSouza, D.O., and deMeis, L. (1996). Regulation of glutamate transport into synaptic vesicles by chloride and proton gradient. Journal of Biological Chemistry 271, 1172611731.

Wu, L.G., Hamid, E., Shin, W., and Chiang, H.C. (2014). Exocytosis and endocytosis: modes, functions, and coupling mechanisms. Annual review of physiology 76, 301-331.

Wucherpfennig, T., Wilsch-Brauninger, M., and Gonzalez-Gaitan, M. (2003). Role of Drosophila Rab5 during endosomal trafficking at the synapse and evoked neurotransmitter release. The Journal of cell biology 161, 609-624.

Yamashita, A., Singh, S.K., Kawate, T., Jin, Y., and Gouaux, E. (2005). Crystal structure of a bacterial homologue of $\mathrm{Na}+/ \mathrm{Cl}-$-dependent neurotransmitter transporters. Nature 437, 215-223.

Yamashita, T., Ishikawa, T., and Takahashi, T. (2003). Developmental increase in vesicular glutamate content does not cause saturation of AMPA receptors at the calyx of Held synapse. The Journal of neuroscience : the official journal of the Society for Neuroscience 23, 3633-3638.

Yernool, D., Boudker, O., Jin, Y., and Gouaux, E. (2004). Structure of a glutamate transporter homologue from Pyrococcus horikoshii. Nature 431, 811-818. 
Zander, J.F., Munster-Wandowski, A., Brunk, I., Pahner, I., Gomez-Lira, G., Heinemann, U., Gutierrez, R., Laube, G., and Ahnert-Hilger, G. (2010). Synaptic and vesicular coexistence of VGLUT and VGAT in selected excitatory and inhibitory synapses. The Journal of neuroscience : the official journal of the Society for Neuroscience 30, 7634-7645.

Zhang, J., Davidson, R.M., Wei, M.D., and Loew, L.M. (1998). Membrane electric properties by combined patch clamp and fluorescence ratio imaging in single neurons. Biophysical journal 74, 48-53.

Zhou, Q., Lai, Y., Bacaj, T., Zhao, M., Lyubimov, A.Y., Uervirojnangkoorn, M., Zeldin, O.B., Brewster, A.S., Sauter, N.K., Cohen, A.E., et al. (2015). Architecture of the synaptotagmin-SNARE machinery for neuronal exocytosis. Nature 525, 62-67.

Zhou, Q., Petersen, C.C., and Nicoll, R.A. (2000). Effects of reduced vesicular filling on synaptic transmission in rat hippocampal neurones. The Journal of physiology 525 Pt 1, 195-206.

Zoccarato, F., Cavallini, L., and Alexandre, A. (1999). The pH-sensitive dye acridine orange as a tool to monitor exocytosis/endocytosis in synaptosomes. Journal of neurochemistry 72, 625633. 


\section{Appendices}

\subsection{Appendix 1: Genotyping}

Genotyping of transgenic mice was performed in collaboration with Brigitte Bargkues (Dep. Neurobiology, MPIbpc, Göttingen, Germany). In order to extract genomic DNA, the ear samples of the spH-21 transgenic mice were first incubated on a thermoshaker ( $750 \mathrm{rpm}$ at $56^{\circ} \mathrm{C}$ ) with $100 \mu \mathrm{g} / \mathrm{ml}$ protein kinase $\mathrm{K}$ (Roche) in lysis buffer ( $\mathrm{NaCl} 200 \mathrm{mM}$, Tris-HCl 10mM, EDTA 5mM, SDS 0.2\% (v/v), pH 8.5) for $2 \mathrm{~h}$. The samples were then centrifuges at $14000 \mathrm{xg}$ for $5 \mathrm{~min}$. The resulting supernatant was thoroughly mixed with $400 \mu \mathrm{l}$ of ice cold ethanol, followed by $20 \mathrm{~min}$ centrifugation at $14000 \mathrm{x} g$. The pellet was then washed with $70 \%$ ethanol, and again centrifuged and dried for $10 \mathrm{~min}$ at room temperature to remove the ethanol. The final pellet containing genomic DNA was dissolved with $30-50 \mu \mathrm{l}$ water at $37^{\circ} \mathrm{C}$ for $30 \mathrm{~min}$ and stored at $4^{\circ} \mathrm{C}$ until use.

Four primers (Table 7-1) for amplifying VMAP2, pHluorin and synaptopHluorin were designed according to (Li et al., 2005) and obtained from Eurofins Scientific. Polymeric chain reaction (PCR) was performed with each genomic DNA preparation as follows:

The reaction mixture for each PCR sample contained:

$1 \mu \mathrm{d}$ dNTP (10 mM, ThermoFsiher Scientific)

$1 \mu \mathrm{l}$ Forward primer $(10 \mu \mathrm{M})$

$1 \mu$ l Reverse primer $(10 \mu \mathrm{M})$

15 ng Genomic DNA

$2.5 \mu \mathrm{l}$ REDTaq Genomic DNA polymerase (Sigma, $1 \mathrm{u} / \mu \mathrm{l}$ )

$5 \mu \mathrm{l}$ 10x PCR reaction buffer containing $\mathrm{MgCl}_{2}$ (Sigma)

Required volume of $\mathrm{H}_{2} \mathrm{O}$ to have $50 \mu \mathrm{l}$

PCR program:

Step 1: $95^{\circ} \mathrm{C} 2 \mathrm{~min}$

Step 2: $95^{\circ} \mathrm{C} 30 \mathrm{~s}$

Step 3: $50^{\circ} \mathrm{C} 30 \mathrm{~s}$

Step 4: $72{ }^{\circ} \mathrm{C} 2 \mathrm{~min}$

30 x cycles of steps $2-4$

Step 5: $72{ }^{\circ} \mathrm{C} 3 \mathrm{~min}$

Step 6: maintaining the sample at $4^{\circ} \mathrm{C}$ 
Table 7-1 List of Primers

\begin{tabular}{|c|c|c|}
\hline Gene & Forward Primer & Reverse Primer \\
\hline VAMP2 & 5'- ctg ccg ccc cgg ccg gcg ag & $5 '-$ tga tga tag gga tga tgg cgc aga tc \\
\hline pHluorin & 5'- ctg gag ttg tcc caa ttc ttg ttg aat tag & 5'- gcc atg tgt aat ccc agc agc tgt tac \\
\hline SynaptopHluorin & $5^{\prime}-$ ctg ccg ccc cgg ccg gcg ag & $5^{\prime}-$ gcc atg tgt aat ccc agc agc tgt tac \\
\hline IL_211 & $5^{\prime}-$ cta ggc cac aga att gaa aga tct & $5^{\prime}-$ gta ggt gga aat tct agc atc atc c \\
\hline
\end{tabular}

1IL_21 gene was used as the control for PCR amplification.

The animals that showed positive amplification for all the three VAMP2, pHluorin and synaptopHluorin genes were considered positive, and used for SV purification. The PCR product was then analyzed by agarose gel electrophoresis, for which 1.5\% agarose (Applichem) gel was prepared in TAE buffer (Tris $20 \mathrm{mM}$, Acetic acid $10 \mathrm{mM}$, EDTA 1 $\mathrm{mM}, \mathrm{pH}$ 8.2-8.4). The PCR samples $(20 \mu \mathrm{l})$ were mixed with the loading dye (Blue/Orange 6X loading dye, Promega) and in parallel with $8 \mu$ l of DNA ladder (GeneRuler $1 \mathrm{~kb}$ DNA ladder, $0.5 \mathrm{mg} / \mathrm{ml}$, Thermo Scientific) were loaded on the gel. The gel was run at $120 \mathrm{~V}$ for $30-45 \mathrm{~min}$ in TAE buffer and then incubated in $0.3 \%(\mathrm{v} / \mathrm{v})$ GelGreen solution (GelGreen Nucleic Acid Gel Stain, 10,000X, Biotium) for 30 min to label the DNA fragment. The stained gel was imaged under UV light (Figure 7-1).

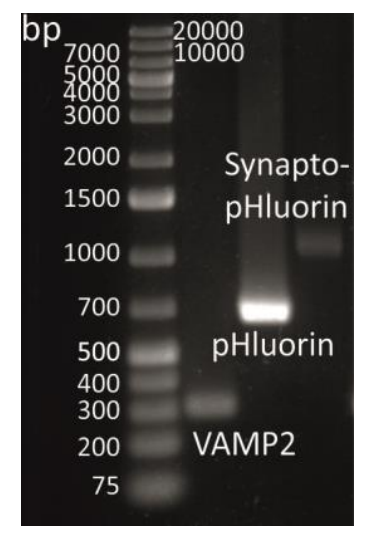

\section{Figure 7-1 Genotyping of spH-21 transgenic mice.}

An example of the results of the agarose gel electrophoresis for a positive animal. Animals that showed positive amplification for all the indictated genes were used for SV purification.

\subsection{Appendix 2: Western Blotting and Dot Blotting}

For the analysis of the proteins, samples were mixed with NuPAGE lithium dodecyl sulfate (LDS) sample buffer (ThermoFsiher Scientific) and loaded on a NuPAGE Bis-Tris 
gradient gel (ThermoFsiher Scientific) which was a pre-caste 4-12\% polyacrylamide gel. Electrophoresis was performed in Tris-MOPS-SDS run buffer (Expedeon) for 30-45 min at $120-150 \mathrm{~V}$.

Western blotting was performed as described by (Towbin et al., 1989). Briefly, separated proteins were transferred from the gel to a nitrocellulose membrane in transfer buffer (Glycine $200 \mathrm{mM}$, Tris 25 mM, SDS 0.04\%, Methanol 20\%) using a tank apparatus under $100 \mathrm{~V}$ constant voltage application for $1 \mathrm{~h}$. The membrane was then blocked by 30 min incubation with blocking buffer (5\% nonfat milk powder in TBST buffer (Tris-HCl $150 \mathrm{mM}, \mathrm{NaCl} 150 \mathrm{mM}$, Tween 20 0.5\% (v/v), pH 7.4)). Next, the membrane was incubated over night with primary antibody diluted in blocking buffer, and washed three times (each 10-15 min) with TBST, followed by incubation with HRPconjugated secondary antibody in blocking buffer and three times washing with TBST. Membrane was then covered with Western Lightening Plus-ECL oxidizing reagent and enhanced luminol reagent (PerkinElmer) with 1:1 ratio and visualized by using chemiluminescence detection on a LumiImager (Boehringer Ingelheim).

In order to perform dot blotting, 3-5 $\mu \mathrm{l}$ of protein samples were directly spotted onto a nitrocellulose membrane and blocking, primary and secondary antibody incubations, as well as protein visualization were performed as described above.

\subsection{Appendix 3: MATLAB Code for Spot Detection}

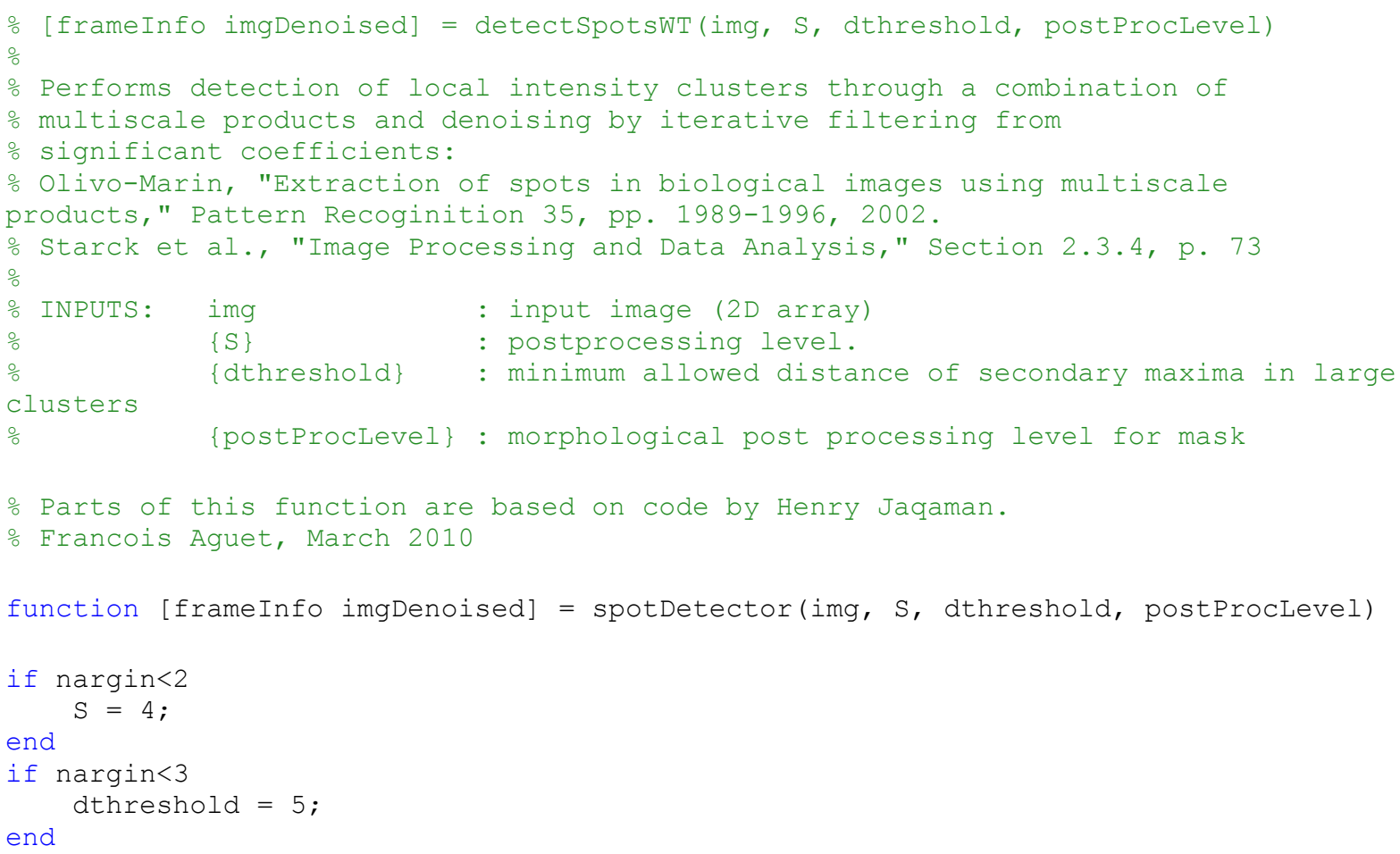




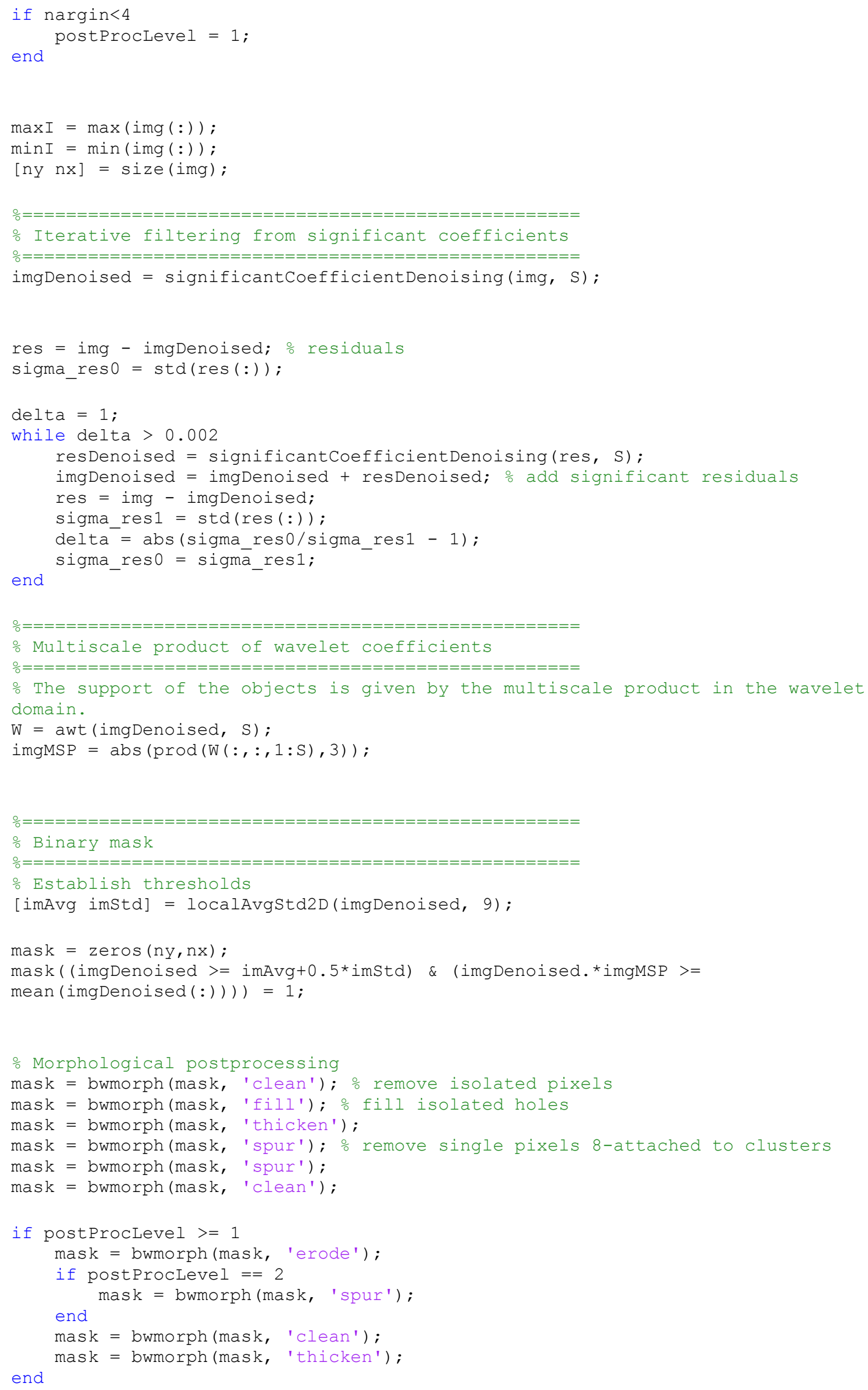




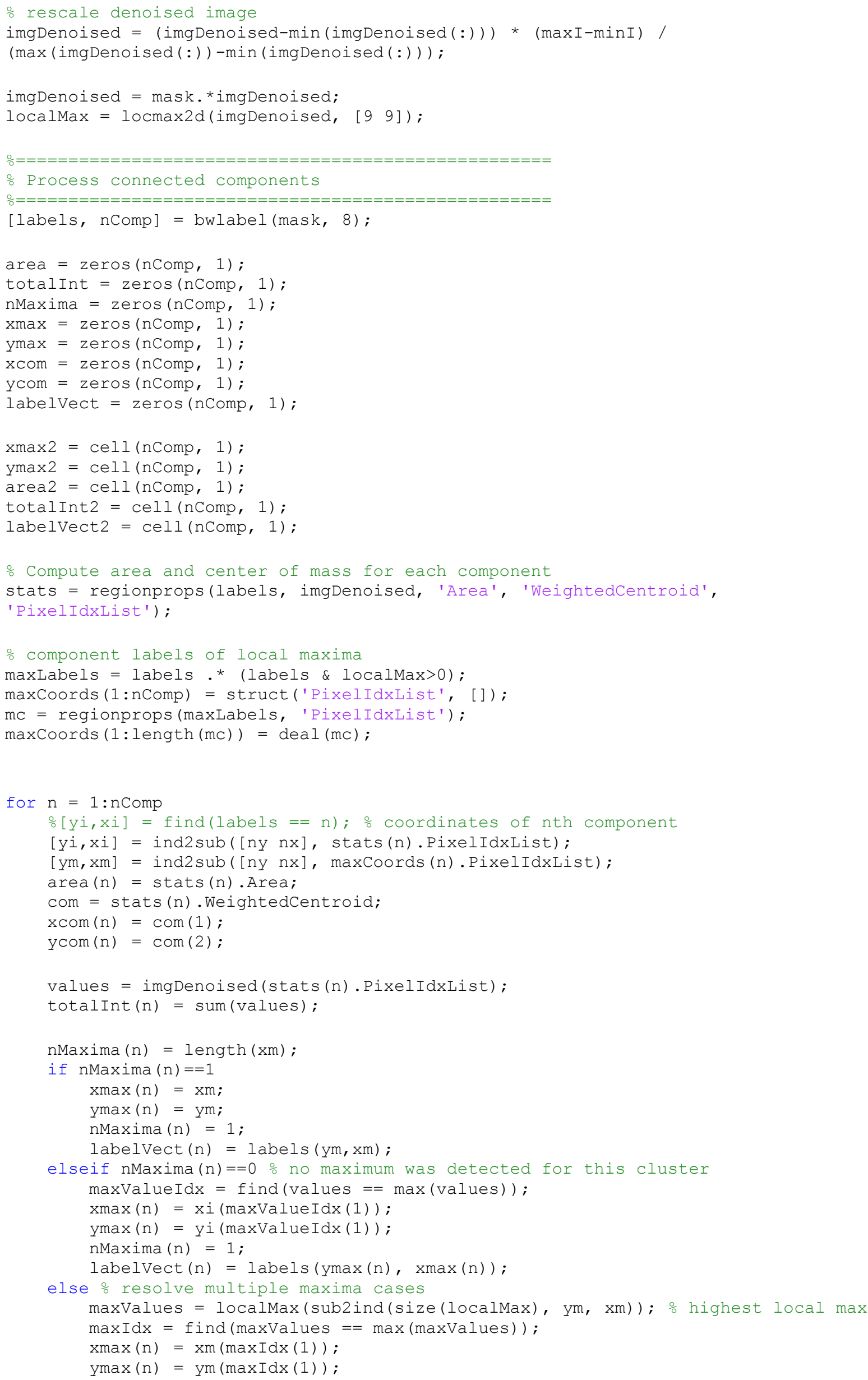




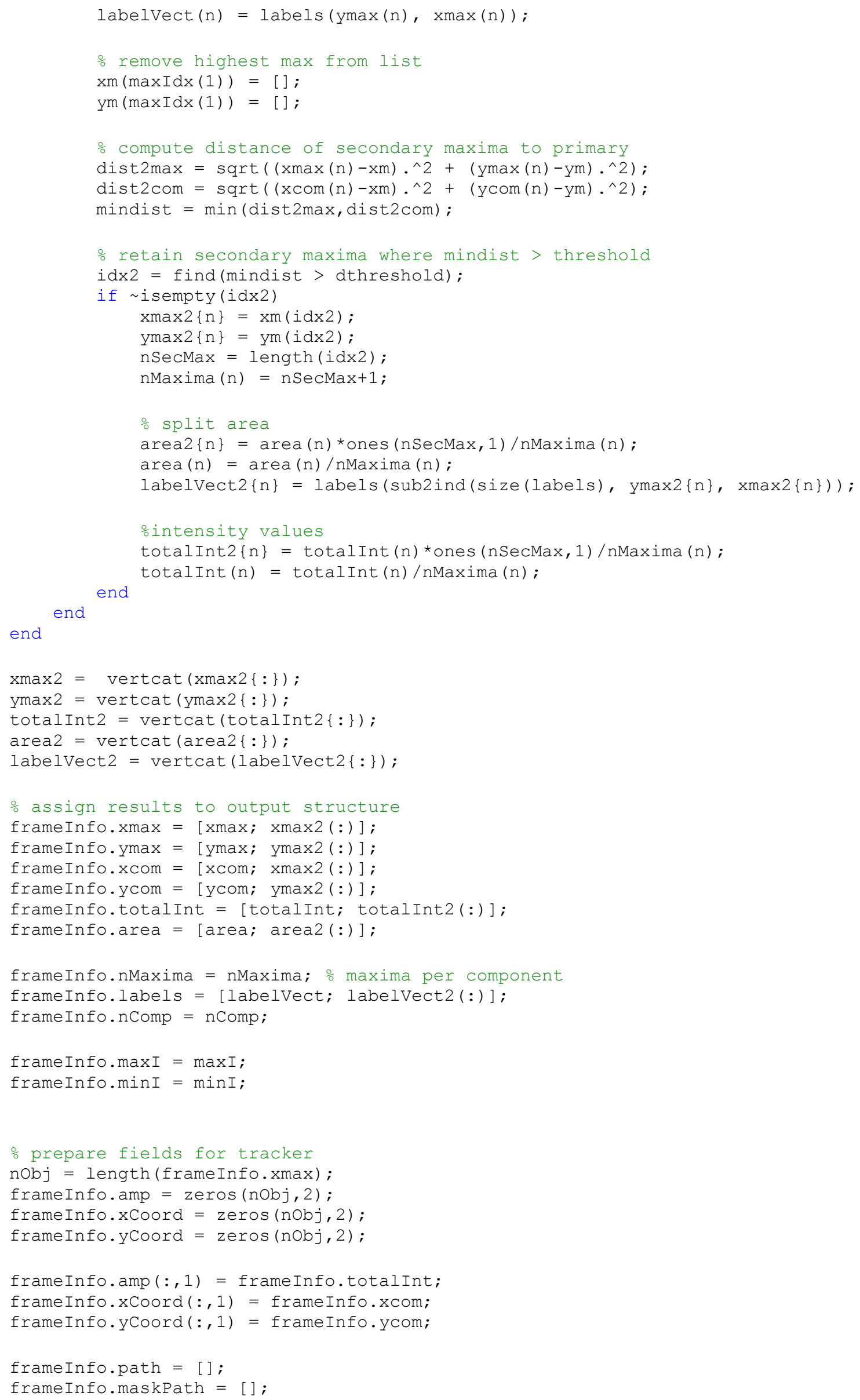




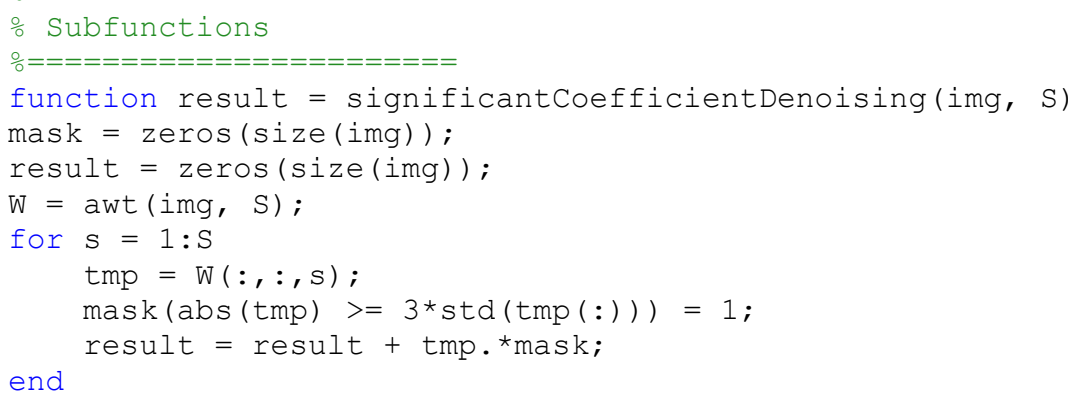




\section{Acknowledgements}

I would like to thank Prof. Dr. Reinhard Jahn not only for his invaluable support and guidance throughout this $\mathrm{PhD}$ project but also because he shares his enthusiasm and love for science with his students.

Many thanks go to Dr. Andrew Woehler for patiently helping me face the challenges and sustain my motivation. I would like to thank Andrew also for his constant support, scientific inputs and technical assistance throughout my $\mathrm{PhD}$ from the first steps of developing the assay to writing the manuscript and the thesis.

I would like to thank the members of my thesis committee Prof. Dr. Silvio O. Rizzoli, Prof. Dr. Tobias Moser and Dr. Geert van den Bogaart for the discussion during the committee meetings and their helpful scientific inputs. I would like to express additional thanks to Geert for his great help with setting up the microscope in the beginning of my $\mathrm{PhD}$ and also for his feedback and criticism throughout writing this thesis. I also especially thank Vedran Vasic for carefully proofreading my PhD thesis.

I would like to express my gratitude to Prof. Dr. Venkatesh N. Murthy and Dr. William J. Tyler for kindly sharing the transgenic mice with us, and also to Dr. Ulrike Teichmann, Sascha Krause and Thomas Gundlach for their help with animal delivery and taking care of the mice. I am grateful to my collaborators; Dr. Julia Preobraschenski, Dr. Dietmar Riedel, Dr. Saheeb Ahmed and Dr. Lars Kastrup for their helpful scientific inputs and technical assistance. I would like to thank Dr. Gotfried Meiskes, Dr. Hans Dieter Schmitt and Elisa Reckmann-Heinrich for their help with lab-related issues. In addition, I thank Brigitte BargKues and Sigrid Schmidt for their technical assistance, and Dr. Javier Matias Hernandez, Dr. John Chua and Dr. Yongsoo Park for their scientific inputs.

I would like to thank all the past and present members of the Neurobiology department for providing a nice and friendly working atmosphere especially Partho, Angel, Dragomir and Beyenech.

I am especially grateful to Mahdokht Kohnasal Nodehi for her close friendship, scientific discussions and for her help with mass spectrometry, and also to Tahere and Sasha for their encouragements when the experiments were not working!

I am very thankful to all my friends in Göttingen, especially to Fatemeh, Elham, Ehsan, Mostafa, Mayur, Bekir, Diana, David, Chaitali, Vinita, Sünke and Alonso for the great time we had and for their support.

Lastly and most importantly, I would like to thank my family without whose support I would have not achieved any of my goals in my life and to whom I dedicate this thesis. 


\section{Curriculum Vitae}

\section{EDUCATION}

Doctor of Philosophy

"Neuroscience", Max-Planck Institute of Biophysical Chemistry, Göttingen, Germany.

Apr.2012-Nov.2015

Master of Science

"Neuroscience", Max-Planck Institute for Experimental Medicine, Göttingen, Germany.

Oct. 2011-Mar. 2012.

Msc/PhD Neuroscience Program

International Max-Planck Research School, Göttingen, Germany.

Sep. 2010-Sep. 2011.

Master of Science

"Biochemistry", Tarbiat Modares University, Tehran, Iran.

Sep. 2009-Sep. 2011.

Bachelor of Science

"Cellular and Molecular Biology-Genetics", Shahed University, Tehran, Iran.

Sep.2005 -Feb.2009.

\section{RESEARCH EXPERIENCE}

PhD Thesis: "A Single-Vesicle Assay to Study the Electrochemical Gradient Regulation in Glutamatergic and GABAergic Synaptic Vesicles"

2012-2015, Prof. Dr. Reinhard Jahn, Max-Planck Institute of Biophysical Chemistry, Göttingen, Germany.

Master's Thesis: "Presynaptic Waveform Change and Neurotransmitter Release in Eag1 Knockout Mice"

2011-2012, Prof. Dr. Luis Pardo, Max Planck Institute for Experimental Medicine, Göttingen, Germany.

Master's Thesis: "Investigation of the role of Arginine 97 in allosteric signal transduction pathway of Methylglyoxal Synthase"

2009-2011, Dr. Khosro Khajeh, Tarbiat Modares University, Tehran, Iran.

Research Intern.: "The Role of the Linker Domain of Synaptobrevin in SNARE-Mediated Liposome Fusion"

2011, Prof. Dr. Reinhard Jahn, Max Planck Institute for Biophysical Chemistry, Göttingen, Germany.

Research Intern.: "Electrophysiological characterization of cells in Neuroblastoma culture" 2011, Dr. Luis Pardo, Max Planck Institute for Experimental Medicine, Göttingen, Germany.

Research Intern.: "The role of HATs and HDACs in Schizophrenia and Aging" 2011, Dr. André Fischer, European Neuroscience Institue, Göttingen, Germany. 
Summer Project: "In vitro effect of antidepressant drugs on murine Macrophages and Lymphocytes"

2009, Dr. Roya Yraei, Shahed University, Tehran, Iran.

Bachelor's Thesis: "Biological Applications of Electrospun Nanofibers"

2009, Dr. Omid Ranaei Siadat, Shahed University, Tehran, Iran.

\section{CONFERENCES}

Horizons in Molecular Biology

Awarded student talk, 'Regulation of neurotransmitter uptake into synaptic vesicles at the single vesicle level', September 2015, Göttingen, Germany.

Gordon Research Conference on 'Synaptic Transmission'

Selected Poster, 'Regulation of neurotransmitter uptake into synaptic vesicles at the single vesicle level'

August 2014, New Hampshire, USA.

Biennial Neurizons Conference

Organizer, May 2013, Gottingen, Germany

FENS satellite event: "Vesicular transporters: old story, fresh look!"

July 2012, Barcelona, Spain.

The Second National Conference on "Biotechnology and its new applications"

Oral Presentation,"Tissue engineering and Electrospinning at Nanobiotechnology".

October 2008, Alzahra University, Tehran, Iran.

$10^{\text {th }}$ International Congress of Immunology and Allergy

Poster, "In vitro effect of antidepressant drugs on murine macrophages and lymphocytes".

May 2010, Tehran, Iran.

\section{PRIZES AND AWARDS}

Awarded poster, Horizons in Molecular Biology Symposium, 2015, Göttingen, Germany.

Awarded Student Talk, Horizons in Molecular Biology Symposium, 2015, Göttingen, Germany.

Awarded poster, Gordon Research Conference on 'Synaptic Transmission', 2015, New Hampshire, USA.

Stipend of the Excellence Foundation for the Promotion of the Max Planck Society, 2012, Göttingen, Germany.

Stipend of the Excellence Foundation for the Promotion of the Max Planck Society, 2010, Göttingen, Germany.

National Foundation of Elites stipend, 2009, Iran.

First Rank in $14^{\text {th }}$ Nationwide Student Olympiad in the field of Biology, July 2009, Tehran, Iran. 
Zohreh Farsi, Julia Preobraschenski, Geert van den Bogaart, Dietmar Riedel, Reinhard Jahn, Andrew Woehler. "Single-vesicle imaging reveals different transport mechanisms between glutamatergic and GABAergic vesicles', in preparation.

Lena Sünke Mortensen, Hartmut Schmidt, Zohreh Farsi, Alonso Barrantes-Freer, Roser Ufartes, Jens Eilers, Takeshi Sakaba, Walter Stühmer, and Luis A. Pardo. "KV10.1 potassium channels modulate presynaptic short-term plasticity at the parallel fibre - Purkinje cell synapse" The Journal of Physiology 593.1, 181-196 (2014).

Javier M. Hernandez, Alexander Stein, Elmar Behrmann, Dietmar Riedel, Anna Cypionka, Zohreh Farsi, Peter J.Walla, Stefan Raunser, Reinhard Jahn. "Membrane fusion intermediates via directional and full assembly of the SNARE complex" Science 22, 336 (2012).

Zohreh Farsi, Helmut Pein, Mohammad Pazhang, Shekoofeh Zareian, Seyed-Omid Ranaei-Siadat, Khosro Khajeh. "Conferral of allostery to Thermus sp. GH5 methylglyoxal synthase by a single mutation" The Journal of Biochemistry 152, 531-8 (2012). 
